\title{
WestVirginiaUniversity
}

THE RESEARCH REPOSITORY @ WVU

Graduate Theses, Dissertations, and Problem Reports

2002

\section{Integrated through -wafer optical monitoring of MEMS for closed -loop control}

Jeremy Michael Dawson

West Virginia University

Follow this and additional works at: https://researchrepository.wvu.edu/etd

\section{Recommended Citation}

Dawson, Jeremy Michael, "Integrated through -wafer optical monitoring of MEMS for closed -loop control" (2002). Graduate Theses, Dissertations, and Problem Reports. 1578.

https://researchrepository.wvu.edu/etd/1578

This Dissertation is protected by copyright and/or related rights. It has been brought to you by the The Research Repository @ WVU with permission from the rights-holder(s). You are free to use this Dissertation in any way that is permitted by the copyright and related rights legislation that applies to your use. For other uses you must obtain permission from the rights-holder(s) directly, unless additional rights are indicated by a Creative Commons license in the record and/ or on the work itself. This Dissertation has been accepted for inclusion in WVU Graduate Theses, Dissertations, and Problem Reports collection by an authorized administrator of The Research Repository @ WVU.

For more information, please contact researchrepository@mail.wvu.edu. 


\title{
Integrated Through-Wafer Optical Monitoring of MEMS for Closed-Loop Control
}

\author{
Jeremy M. Dawson \\ B.S.E.E, M.S.E.E., West Virginia University \\ Dissertation submitted to the \\ College of Engineering and Mineral Resources at \\ WEST VIRGINIA UNIVERSITY \\ in partial fulfillment of the requirements for the degree of \\ Doctor of Philosophy in Electrical Engineering
}

\author{
Committee Members: \\ Larry A. Hornak, Ph.D., Chair \\ Parviz Famouri, Ph.D. \\ Mark Jerabek, Ph.D. \\ Kathleen Meehan, Ph.D. \\ Department of Computer Science and Electrical Engineering \\ Charter Stinespring, Ph.D. \\ Department of Chemical Engineering
}

\section{Morgantown, West Virginia}

2002

Keywords: Microelectromechanical systems, integrated optical monitoring, closed-loop control 


\title{
ABSTRACT \\ Integrated Through-Wafer Optical Monitoring of MEMS for Closed-Loop Control
}

\author{
Jeremy M. Dawson
}

\begin{abstract}
Current trends in many microelectronic systems show an increased use of microelectromechanical systems (MEMS) to perform a variety of tasks. The increased market for MEMS has led to microsystem technologies being employed in physically demanding environments and safety critical applications. This creates the need for higher degrees of certainty in MEMS operation, especially in systems that contain drive components operating under time varying load conditions. Situations such as these give rise to the need for detailed knowledge of the operational states of MEMS over the lifetime of the device, as well as device fault detection. Accurately obtaining this information by a means decoupled from the system shows the potential to further enable both complex and simple MEMS, and allows for the application of closed-loop control. Preliminary through-wafer optical monitoring research efforts have shown that through-wafer optical probing is suitable for characterizing and measuring the behavior of lateral harmonic oscillators.

This presentation will discuss research undertaken to establish integrated optical monitoring (IOM) for closed-loop control. Design of the optical microprobe setup, as well as device geometry, were completed to achieve a through-wafer optical signal with increased positional resolution and mechanical stability. Successful linear closed-loop control results achieved using the redesigned probe setup and devices will be presented. Increased displacement information in the optical output waveform is needed for the successful application of more robust, nonlinear control routines. Theoretical optical output field intensity studies are presented and compared with experimental output waveforms, showing a positional resolution of $2 \mu \mathrm{m}$ using grating structures. Initial binary Fresnel diffractive optical microelement design layout, fabrication parameters, and testing results will be given as well for implementation of a fully integrated optical monitoring system.
\end{abstract}




\section{Acknowledgements}

I would like to thank my committee members Dr. Larry Hornak, Dr. Parviz Famouri, Dr. Mark Jerabek, Dr. Kathleen Meehan, and Dr. Charter Stinespring for the guidance and support that they provided over the course of this research work. I would also like to thank members of the WVU Electromechanical Systems Laboratory, Dr. Jingdong Chen, Limin Wang, and Juchirl Park for their contributions of closed-loop control and system modeling results, and other members of the CSEE MEMS research group: Bill McCormick, Scott Rittenhouse, and Chamila Tissera. Special thanks to Dr. Steve McGinnis, Dr. Kolin Brown, Paul Sines, Chris Garman, Srikanth Nistala, and Carlo Da Cuhna for lab assistance and moral support.

I would especially like to thank my family and friends for their prayers, support, and patience during the entirety of my graduate career. Last, but not least, I would like to thank God for walking beside me during this and all of life's difficulties, both large and small. 


\section{Contents}

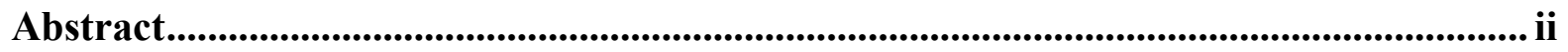

Acknowledgements ............................................................................................................................ iii

Contents ............................................................................................................................... iv

List of Figures........................................................................................................................... vii

List of Tables .................................................................................................................................. xiv

Chapter 1 Introduction.................................................................................................... 1

1.1 Current Trends in MEMS Technology ………………........................................ 2

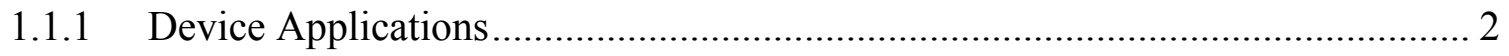

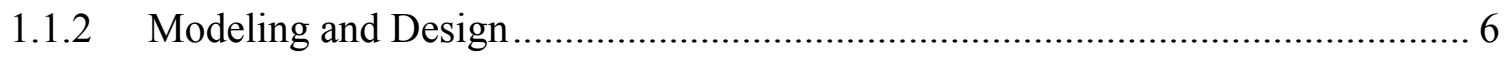

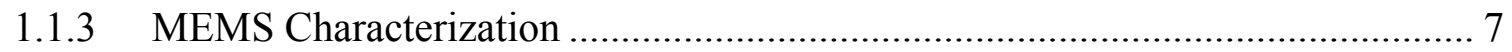

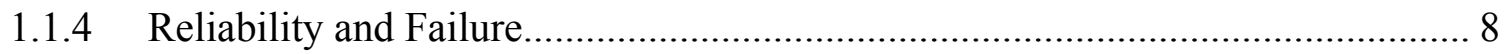

1.2 Integrated Optical Monitoring and Control Exploration ………………................... 10

1.2.1 Preliminary Through-Wafer Monitoring Results................................................ 10

1.2.2 Through-Wafer Optical Probe System Redesign................................................ 18

1.2.2.1 Integrated Fresnel Zone Plate Lenses............................................................ 19

1.2.2.2 Vibration Isolation............................................................................. 22

1.2.3 Absolute Position Determination.................................................................. 22

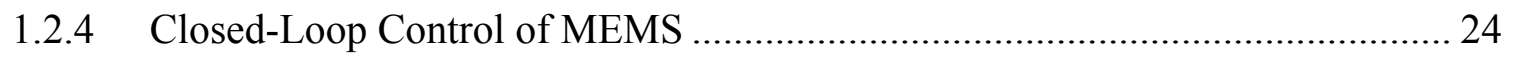

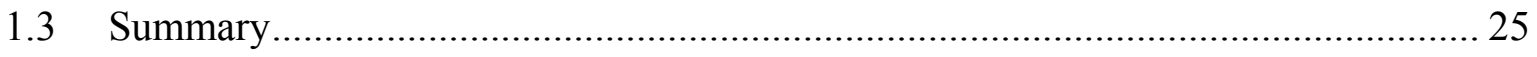

Chapter 2 Theory ......................................................................................................................... 27 
2.1 MEMS System Model Development................................................................ 27

2.2 Examination of Diffraction Effects Caused by MEMS Device Stage Features ........ 34

2.3 Fresnel Lens Zone Pattern Determination .............................................................. 37

2.4 Fiber-to-Fiber and Laser Diode-to-Fiber Coupling Efficiency Examination............. 42

2.4.1 Optical Output Element ................................................................................. 43

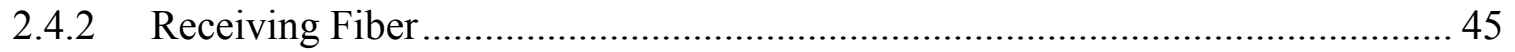

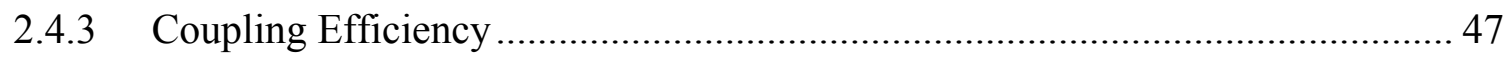

2.4.4 Complex Error Function ............................................................................. 49

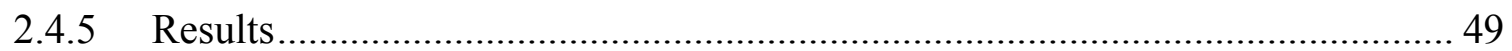

Chapter 3 MEMS Lateral Comb Resonator and Optical Probe System Design ............. 52

3.1 MEMS Design .............................................................................................. 52

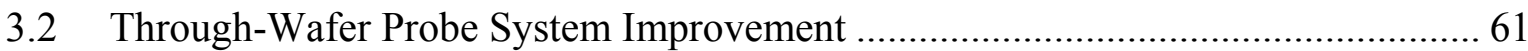

Chapter 4 Through-Wafer Diffraction Study ............................................................................ 65

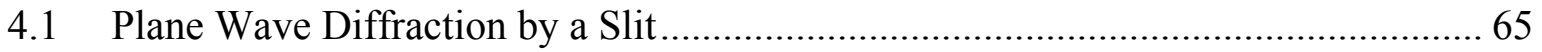

4.2 Plane Wave Diffraction by a Rectangular Aperture ................................................... 70

4.3 Plane Wave Diffraction Through a Grating …………………………………....... 77

4.4 Gaussian Beam Diffraction Through the Device Stage............................................ 80

4.4.1 Single Opening Diffraction Region .............................................................. 80

4.4.2 Closed-Loop Control Results......................................................................... 82

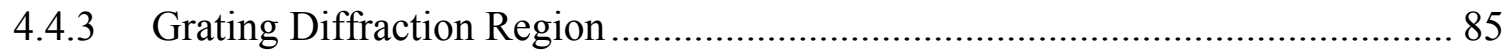

4.4.4 Theoretical and Experimental Grating Signal Examination ............................... 87

Chapter 5 Diffractive Optical Plane Layout and Integrated Fresnel Lens Design, Fabrication, and Testing .................................................................................................. 113

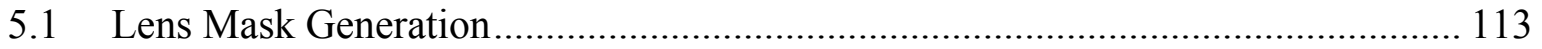

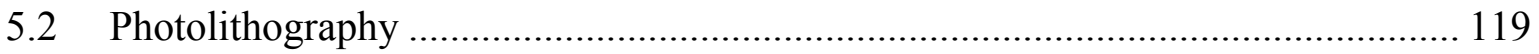

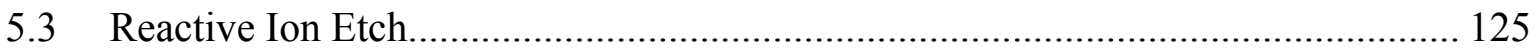

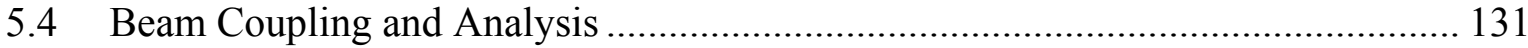

5.5 MEMS Monitoring Using the Lens Substrate .................................................. 136

Chapter 6 Conclusions.................................................................................................................. 140

6.1 Through-Wafer Diffraction Study Results ……………………………………..... 140

6.2 Integrated Binary Fresnel Lens Conclusions............................................................ 142 
Appendix A Lens Design and Optical Analysis Programs............................................... 144

Bibliography .................................................................................................................... 177 


\section{List of Figures}

Figure 1.1: The Sandia Microengine. Two sets of linked comb drives (a) are used to turn a 'drive gear' (b). A portion of a 24-bit mechanical lock that uses microengines to move other micromechanical elements (c) (Sandia National Laboratories) [4] ..................................... 2 Figure 1.2: Translational actuation MEMS devices: a MUMPs fabricated lateral comb resonator (a), and a dual-comb arrangement used to power the Sandia Microengine (b)....... 3 Figure 1.3: Torsional actuation MEMS devices: the WaveStar Lambda Router (a) (Lucent Technologies) [8], and two unit cells of the DMD projection display device (b) (Texas Instruments) [9].

Figure 1.4: Rotational actuation MEMS devices: a MUMPs fabricated salient-pole sidedrive micromotor (a) and the Sandia Microengine drive gear (b) and larger gears and microstructures driven by it (c)

Figure 1.5: MUMPs fabricated lateral comb resonator (a). The initial through-wafer bulk optical monitoring scheme (b). IR image of focused through-wafer spot (c). 11

Figure 1.6: Through-wafer scan of a stationary lateral comb resonator stage. (a) Scan area, cross section view for thickness illustration, and spot location at $0 \mathrm{~V}$ for probing a powered device (direction of motion indicated). (b) Scan data with $47 \mu \mathrm{m}$ detector fiber and (c) $8 \mu \mathrm{m}$ fiber.

Figure 1.7: InGaAs photoreceiver input/output relationship. 14

Figure 1.8: Through-wafer optical signal in volts and calculated static deflection versus applied voltage 
Figure 1.9: Drive voltage (I), normalized stage position (II), and through-wafer detector

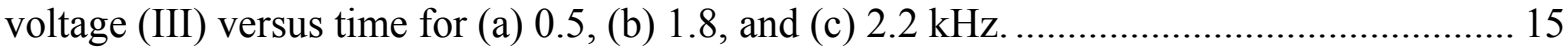

Figure 1.10: Through-wafer optical signal modulation depth versus frequency................. 16

Figure 1.11: Illustration and loss analysis of proposed IOM scheme............................... 18

Figure 1.12: Representation of a $10^{\circ}$ off-axis binary ZPL. ........................................... 20

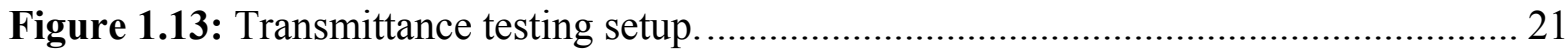

Figure 1.14: Single opening through-wafer probing MEMS device. .............................. 23

Figure 1.15: Grating structure through-wafer probing area........................................... 23

Figure 1.16: Research task flow illustration. ............................................................ 25

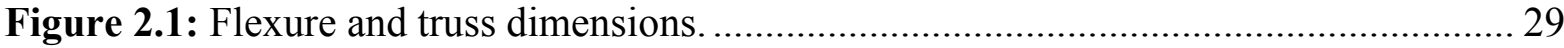

Figure 2.2: Theorized types of vertical stage motion: (a) raising motion with torsional bending, (b) parallel raising motion, and (c) parallel raising and lowering.......................... 32

Figure 2.3: Angular incidence interaction at a planar boundary resulting in multiple

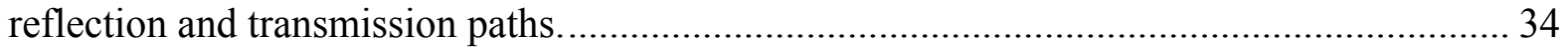

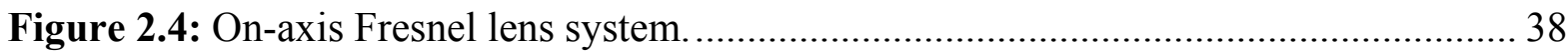

Figure 2.5: (a) Zone pattern cross-section illustrating phase levels. (b) Wavelength to phase

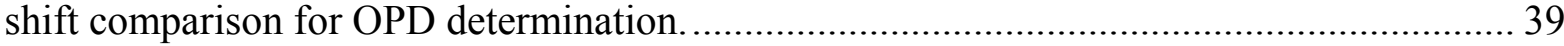

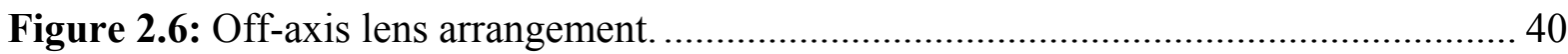

Figure 2.7: Relaxed input illustration for off-axis lenses. .......................................... 41

Figure 2.8: Spot spreading due to comatic aberration. ................................................ 41

Figure 2.9: Circular Gaussian Beam Intensity at $100 \mu \mathrm{m}$. .............................................. 43

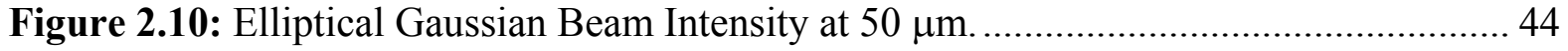

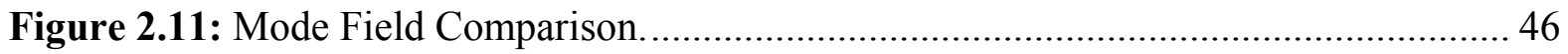

Figure 2.12: Fiber-to-fiber coupling efficiency as a function of $z$-axis separation. ............. 50

Figure 2.13: Laser diode-to-fiber coupling efficiency as a function of $z$-axis separation..... 50

Figure 2.14: Comparison of fiber-to-fiber and LD-to-fiber coupling efficiency................. 51

Figure 3.1: Basic comb resonator geometry. .............................................................. 53

Figure 3.2: Single opening (a) and grating (b) through-wafer optical monitoring structures.

Figure 3.3: Resonator generator geometry. .......................................................... 55

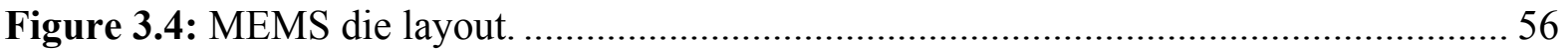


Figure 3.5: MUMPS mask levels and their corresponding cross sections: (a) POLY 0, (b) ANCHOR 1, and (c) DIMPLE

Figure 3.6: POLY 1 layer: (a) basic device geometry, (b) single opening and cross section,

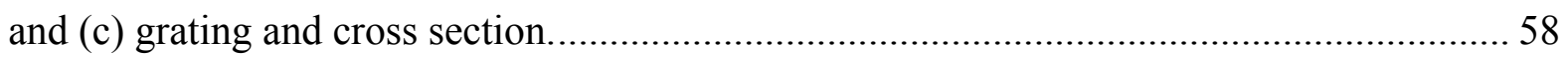

Figure 3.7: MUMPS mask levels and their corresponding cross sections: (a) POLY 1- POLY

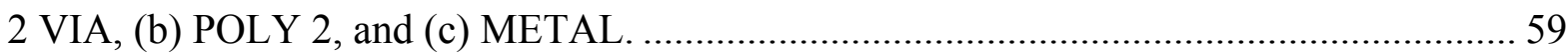

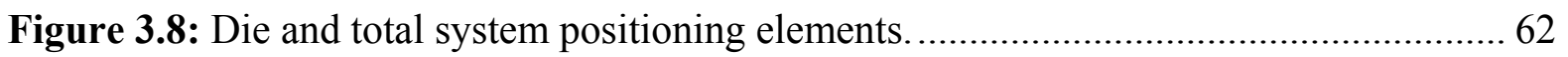

Figure 3.9: Probe beam and detector fiber adjustment. .................................................. 63

Figure 3.10: Close-up of die package and detector fiber............................................ 64

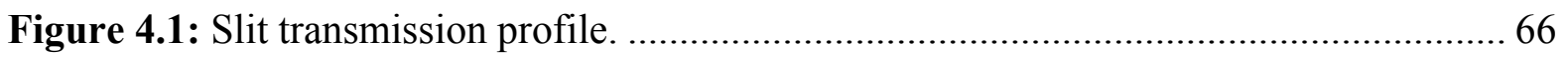

Figure 4.2: Aperture shadowed beam pattern.............................................................. 66

Figure 4.3: Slit effected output beam intensity profile, $\mathrm{N}_{\mathrm{F}}=90, \mathrm{~d}=0.85 \mu \mathrm{m} \ldots \ldots \ldots \ldots \ldots \ldots . . .67$

Figure 4.4: Slit effected output beam intensity profile, $\mathrm{N}_{\mathrm{F}}=10, \mathrm{~d}=7.63 \mu \mathrm{m} \ldots \ldots \ldots \ldots \ldots \ldots . . . . . .67$

Figure 4.5: Slit effected output beam intensity profile, $\mathrm{N}_{\mathrm{F}}=1, \mathrm{~d}=76.33 \mu \mathrm{m} \ldots \ldots \ldots \ldots \ldots \ldots . . .68$

Figure 4.6: Slit effected output beam intensity profile, $\mathrm{N}_{\mathrm{F}}=0.5, \mathrm{~d}=152.67 \mu \mathrm{m} \ldots \ldots \ldots \ldots \ldots . . .68$

Figure 4.7: Slit effected output beam intensity profile, $\mathrm{N}_{\mathrm{F}}=0.5, \mathrm{~d}=76.33 \mu \mathrm{m} \ldots \ldots \ldots \ldots \ldots . . .69$

Figure 4.8: Program output errors: (a) $\mathrm{N}_{\mathrm{F}}=0.1$, not enough zeros included in arrays for convolution (b) $\mathrm{N}_{\mathrm{F}}=10$, calculation loop step size too small.

Figure 4.9: Rectangular aperture pupil function (a) and its corresponding aperture shadowed

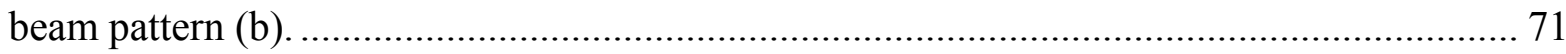

Figure 4.10: Beam intensity at $\mathrm{N}_{\mathrm{F}}=1, \mathrm{~d}=19 \mu \mathrm{m}$ (a), side view (b), and top view (c)....... 72

Figure 4.11: Beam intensity at $\mathrm{N}_{\mathrm{F}}=0.5, \mathrm{~d}=38.2 \mu \mathrm{m}$ (a), side view (b), and top view (c)... 73

Figure 4.12: Beam intensity at $\mathrm{N}_{\mathrm{F}}=0.25, \mathrm{~d}=76.3 \mu \mathrm{m}$ (a), side view (b), and top view (c). 74

Figure 4.13: Beam intensity at $\mathrm{N}_{\mathrm{F}}=0.1, \mathrm{~d}=190.8 \mu \mathrm{m}$ (a), side view (b), and top view (c). 75

Figure 4.14: Beam intensity at $\mathrm{N}_{\mathrm{F}}=0.05, \mathrm{~d}=381.7 \mu \mathrm{m}(\mathrm{a})$, side view (b), and top view (c). 76

Figure 4.15: Grating effected output beam intensity profile, $\mathrm{N}_{\mathrm{F}}=90, \mathrm{~d}=0.85 \mu \mathrm{m} . \ldots \ldots \ldots . .77$

Figure 4.16: Grating effected output beam intensity profile, $\mathrm{N}_{\mathrm{F}}=10, \mathrm{~d}=7.63 \mu \mathrm{m} . \ldots \ldots \ldots . .78$

Figure 4.17: Grating effected output beam intensity profile, $\mathrm{N}_{\mathrm{F}}=1, \mathrm{~d}=76.33 \mu \mathrm{m}$......... 78

Figure 4.18: Grating effected output beam intensity profile, $\mathrm{N}_{\mathrm{F}}=0.5, \mathrm{~d}=153.67 \mu \mathrm{m} . \ldots \ldots .79$ 
Figure 4.19: Grating effected output beam intensity profile, $\mathrm{N}_{\mathrm{F}}=0.1, \mathrm{~d}=763.36 \mu \mathrm{m} . \ldots \ldots .79$

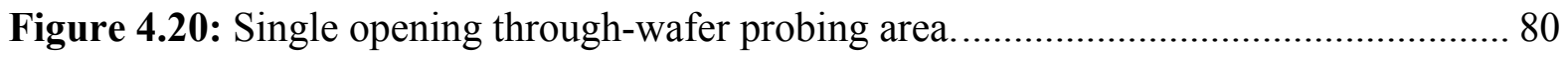

Figure 4.21: Through wafer diffraction pattern of a MEMS lateral comb resonator translation stage with a single single-opening for $8 \mu \mathrm{m}$ of travel and corresponding stage cross sections (to scale) (a)-(e). Detector intensity for the same range of motion (f)-(j)...... 81

Figure 4.22: Active impulse disturbance damping (a) with close-up of single pulse response

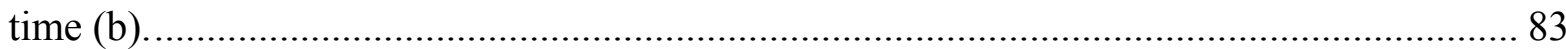

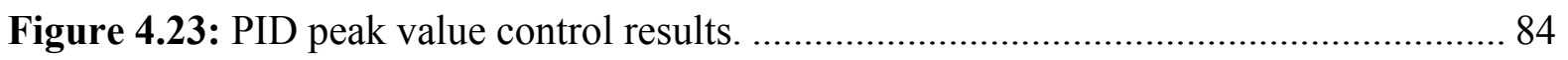

Figure 4.24: Grating structure through-wafer probing area........................................... 85

Figure 4.25: Through wafer diffraction pattern of a MEMS lateral comb resonator translation stage with a diffraction grating for $4 \mu \mathrm{m}$ of travel and corresponding stage cross sections (not to scale) (a)-(e). Detector intensity for the same range of motion (f)-(j).......... 86

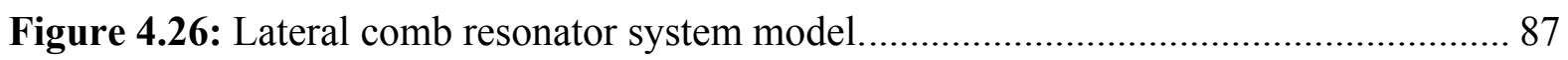

Figure 4.27: Optical output comparison at $1000 \mathrm{~Hz}$ : (a) theoretical results and (b)

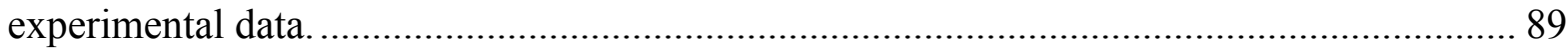

Figure 4.28: Optical output comparison at $1500 \mathrm{~Hz}$ : (a) theoretical results and (b) experimental data.

Figure 4.29: Optical output comparison at $2000 \mathrm{~Hz}$ : (a) theoretical results and (b)

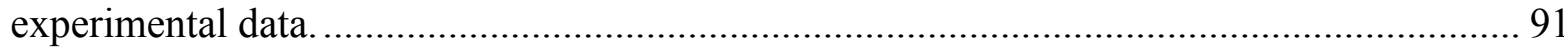

Figure 4.30: Optical output comparison at $2200 \mathrm{~Hz}$ : (a) theoretical results and (b)

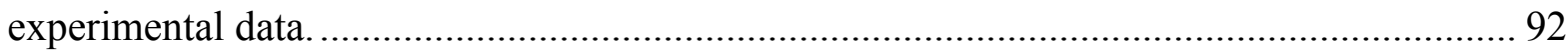

Figure 4.31: Optical output comparison at $2400 \mathrm{~Hz}$ : (a) theoretical results and (b) experimental data.

Figure 4.32: Optical output comparison at $2600 \mathrm{~Hz}$ : (a) theoretical results and (b) experimental data.

Figure 4.33: Optical output comparison at $2700 \mathrm{~Hz}$ : (a) theoretical results and (b) experimental data.

Figure 4.34: Optical output comparison at $2800 \mathrm{~Hz}$ : (a) theoretical results and (b) experimental data. 96

Figure 4.35: Optical output comparison at $2900 \mathrm{~Hz}$ : (a) theoretical results and (b) experimental data. 
Figure 4.36: Optical output comparison at $3000 \mathrm{~Hz}$ : (a) theoretical results and (b)

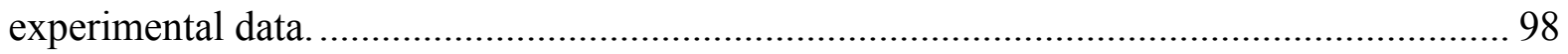

Figure 4.37: Optical output comparison at $3100 \mathrm{~Hz}$ : (a) theoretical results and (b)

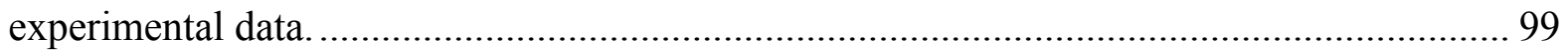

Figure 4.38: Optical output comparison at $3200 \mathrm{~Hz}$ : (a) theoretical results and (b) experimental data.

Figure 4.39: Optical output comparison at $3300 \mathrm{~Hz}$ : (a) theoretical results and (b) experimental data.

Figure 4.40: Optical output comparison at $3400 \mathrm{~Hz}$ : (a) theoretical results and (b) experimental data.

Figure 4.41: Optical output comparison at $3600 \mathrm{~Hz}$ : (a) theoretical results and (b) experimental data.

Figure 4.42: Optical output comparison at $3800 \mathrm{~Hz}$ : (a) theoretical results and (b) experimental data.

Figure 4.43: Optical output comparison at $4000 \mathrm{~Hz}$ : (a) theoretical results and (b) experimental data.

Figure 4.44: Optical output comparison at $4500 \mathrm{~Hz}$ : (a) theoretical results and (b) experimental data.

Figure 4.45: Optical output comparison at $5000 \mathrm{~Hz}$ : (a) theoretical results and (b) experimental data. 107

Figure 4.46: Change in transmission due to variation in oxide gap thickness 110

Figure 4.47: Oxide gap variation at $2.9 \mathrm{kHz}$ (a) and the resulting transmission intensity change (b).

Figure 4.48: The theoretical optical output waveform resulting from vertical stage motion (a) and lateral displacement (b). 111

Figure 4.49: Spot interaction with solid stage areas due to large spot size and increased lateral displacement (a) and angular stage alignment (b).

Figure 5.1: Lens mask layout: (a) top lens mask, (b) bottom lens mask, (c) both masks superimposed. Bottom lenses are denoted with an ' $\mathrm{x}$ '. Propagation lengths indicated are for 5 internal reflections at the given angle. 
Figure 5.2: Sample lens geometries: (a) 10-2-0, (b) 10-2-10, (c) 10-2-15, (d) 5-2-10, (e) 5-215. Line roughness and secondary diffraction patterns are a result of the zoom level of the layout software. 116

Figure 5.3: Secondary intensities caused by internal reflections in the air gap..... 117

Figure 5.4: Comparison of primary off-axis probe intensity and secondary intensities caused by oxide gap reflections. 118

Figure 5.5: Intensity separation distance for various input angles. Separation distance change for a gap variation of 0.5 microns is included 118

Figure 5.6: Illustration of partial pattern development due to exposure type. 120

Figure 5.7: Photoresist pattern achieved with vacuum contact exposure and PR lip removal: (a) large features and (b) small lines. 121

Figure 5.8: Photoresist de-adhesion. 122

Figure 5.9: Thin photoresist lines due to underexposure. 123

Figure 5.10: Successful photoresist patterning on 3 in. quartz: (a) small radii rings, (b) medium radii rings, and (c) large radii rings.

Figure 5.11: Successful etch patterning on 3 in. quartz: (a) small radii rings, (b) medium radii rings, and (c) large radii rings. 128

Figure 5.12: Completed lens substrate with top and bottom lenses. Spots are diffracted and focused light from the camera flash. 129

Figure 5.13: Lens substrate process flow 130

Figure 5.14: Fresnel lens analysis setup. 131

Figure 5.15: Beam coupling using an early AZ5214 photoresist pattern. 132

Figure 5.16: Etch effect on transmitted beam intensity: (a) un-etched substrate area and (b) etched substrate area. 132

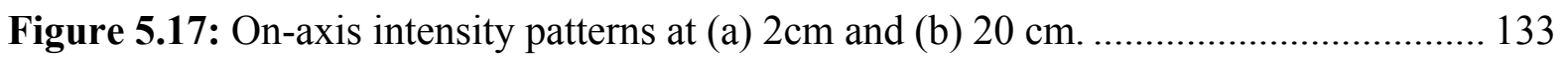

Figure 5.18: Coupled off-axis beam intensity at $1 \mathrm{~cm}$. 134

Figure 5.19: Angled view of coupled off-axis beam intensity at $1 \mathrm{~cm}$. Internal reflections are readily visible. 135

Figure 5.20: Comatic aberration of the output beam at (a) $20 \mathrm{~cm}$ and (b) $40 \mathrm{~cm}$. Secondary diffraction intensities of the input beam are visible. 136

Figure 5.21: Microoptical reflective device monitoring setup. 137 
Figure 5.22: Optical microprobe setup with lens substrate added................................. 137

Figure 5.23: Close-up of the package and lens substrate arrangement........................... 138

Figure 5.24: The lens substrate in use, illuminating a device on the MEMS die. ............. 139

Figure 5.25: A single-opening lateral comb resonator illuminated by the output beam of the

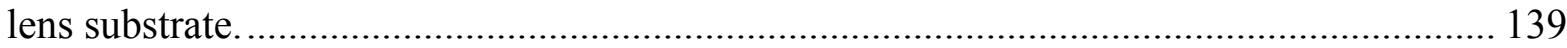




\section{List of Tables}

Table 1.1: Percentage Transmission of MUMPs MEMS films at 1310nm. ...................... 13

Table 2.1: Zone radius equations for various phase shifts............................................ 39

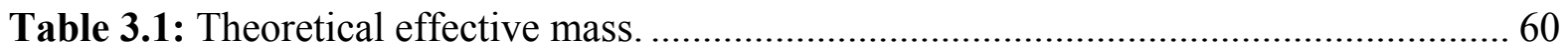

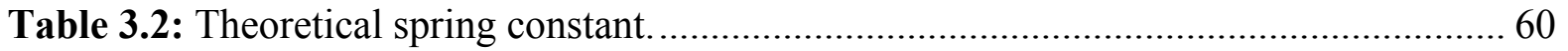

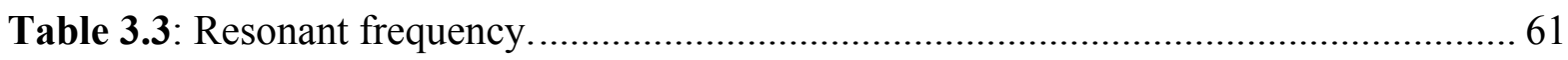

Table 4.1: Comparison of theoretical and experimentally determined displacement values.

Table 5.1: Lens types and the smallest feature size on each lens. ................................. 114

Table 5.2: Comparison of desired and photoresist linewidths..................................... 125

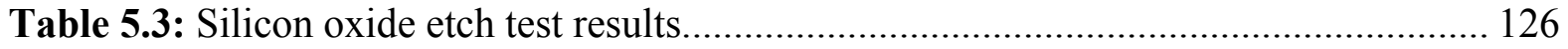

Table 5.4: Etch depths for quartz. All times performed with fluoroform at $30 \mathrm{mT}, 100 \mathrm{sccm}$,

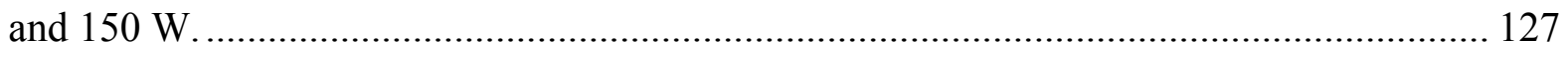

Table 5.5: Comparison of desired and etched linewidths............................................. 129 


\section{Chapter 1 Introduction}

Current trends in many microelectronic systems show an increased use of microelectromechanical systems (MEMS) to perform a variety of tasks. The application of MEMS gives the advantages of mass production and high packing density offered by current CMOS technology, while at the same time producing mechanical structures that are small, lightweight, and offer low power operation [1]. Since the first discussion of using silicon as a mechanical material by Peterson in 1982 [2], MEMS have developed into a multibilliondollar industry. MEMS can be used as sensors and, also, as actuators that exhibit a variety of motion, such as translational, torsional, and rotational movement, as well as combinations of these. MEMS actuators also include microfluidic devices, developed to transfer small amounts of liquid in microchannels. Although currently dominated by sensors, one estimate shows that actuators will account for two-thirds of the MEMS market by 2005 [3].

The increased market for MEMS has led to microsystem technologies being employed in physically demanding environments and safety critical applications, creating the need for

higher degrees of certainty in MEMS operation. Complex MEMS have been developed in which a number of micromechanical elements are linked to achieve a specific mechanical output function (Figure 1.1 [4]). This can result in drive components operating under time varying load conditions [5]. Situations such as these give rise to the need for detailed knowledge of the operational states of MEMS over the lifetime of the device, as well as the determination of device failure, both partial and catastrophic. Accurately obtaining this 
information by a means decoupled from the system shows the potential to further enable both complex and simple MEMS and allows for the application of closed-loop control routines. Since basic MEMS operation involves common electro-mechanical principles, knowledge of the positional state of these systems can be used to apply control that is highly flexible, and can be optimized to provide enhanced performance under various load and application conditions.

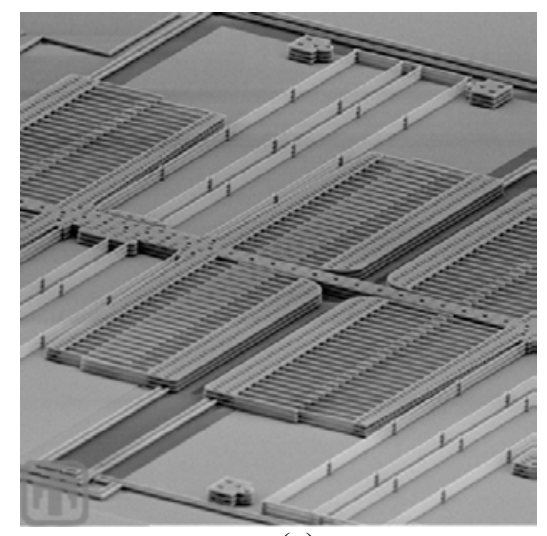

(a)

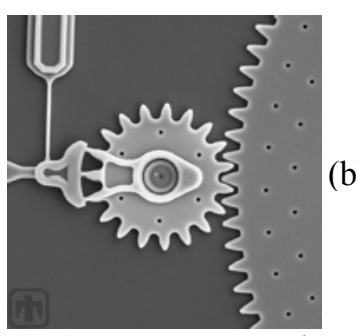

Figure 1.1: The Sandia Microengine. Two sets of linked comb drives (a) are used to turn a 'drive gear' (b). A portion of a 24-bit mechanical lock that uses microengines to move other micromechanical elements (c) (Sandia National Laboratories) [4].

\subsection{Current Trends in MEMS Technology}

\subsubsection{Device Applications}

In order to understand the context in which this research is being done and its range of impact, a brief representative review of MEMS microactuator technology is given. The wide variety of MEMS actuation applications, in both arrayed and single device systems, motivates the need for a means of validation and verification of device operation. 


\section{Translational MEMS}

One of the most common types of MEMS devices is those of the translational variety. One such device, the lateral comb resonator, has been employed in many applications where lateral movement is needed. This device often has two sets of stationary comb fingers interleaved with comb fingers on both sides of a translation stage situated between the stationary, or stator, combs. When acting as an actuator, these interleaved fingers cause an electrostatic force when a voltage is applied. To counteract this force, a mechanical force is provided by spring structures, or flexures, attached to the translation stage. The combination of these two forces allows the stage to move in one direction and then return to its original position when the voltage is removed. For translational MEMS sensors, such as accelerometers, motion causes the fingers to be more interleaved, creating more capacitance. This capacitance change can be calibrated to correspond to a certain degree of acceleration or deceleration. The flexures return the stage to its at-rest position after the motion ceases. Figure 1.2 shows an example of a translational MEMS device.
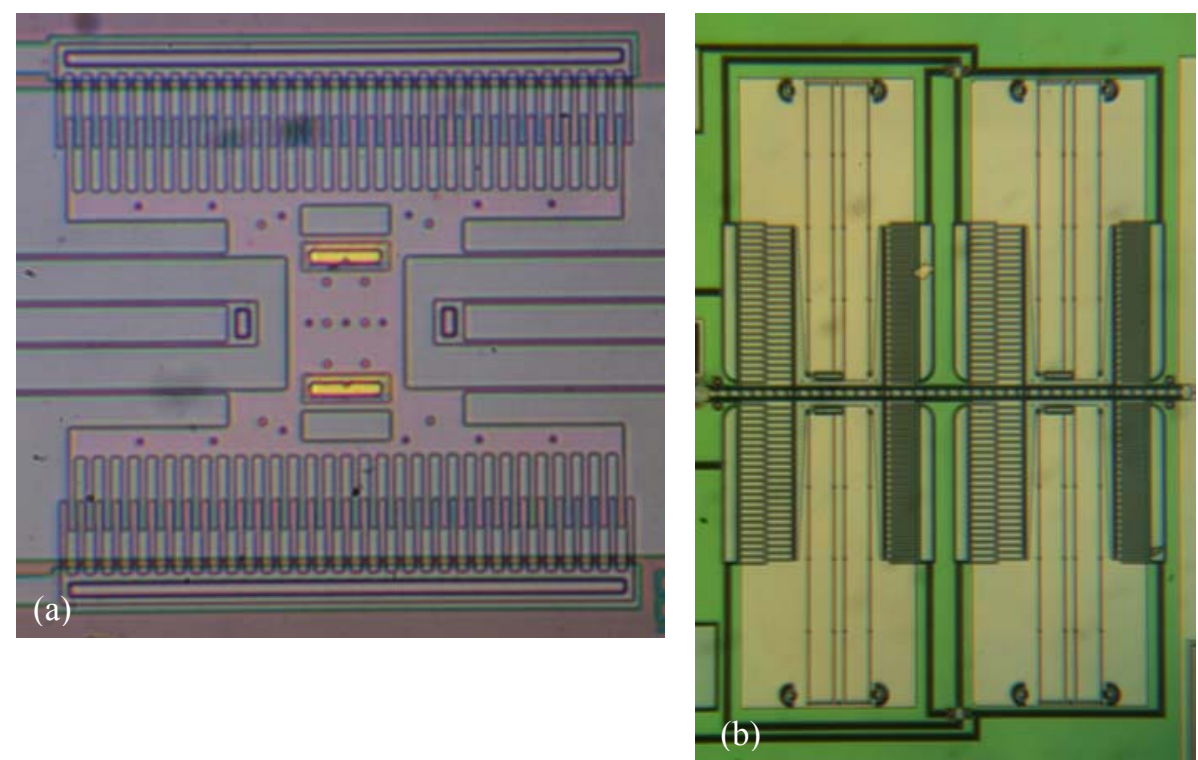

Figure 1.2: Translational actuation MEMS devices: a MUMPs fabricated lateral comb resonator (a), and a dual-comb arrangement used to power the Sandia Microengine (b).

Translational actuators have been utilized in many applications, ranging from fiber optical switching and signal attenuation to micro-scale spectrometry [6]. Also known as resonator devices due to their operational characteristics, this type of device has also been 
used in many applications involving the actuation of other micromechanical components in MEMS. One such system employs translation stages to position components on a surfacemicromachined free-space optical bench [7]. In this application, hinge structures, microfabricated mirrors, and micro-Fresnel lenses combine to form a microoptoelectromechanical (MOEM) system that provides scanning and focusing of a beam from a semiconductor laser.

\section{Torsional/Vertical Motion MEMS}

Micromirror arrays utilize MEMS technology in order to scan or modulate light. The first major application of linear arrays of such mirrors was for light modulation in printers. Recently, the optical communications industry has started utilizing large arrays of these mirrors to create high-bandwidth optical cross connects, such as the WaveStar LambdaRouter developed by Lucent Technologies, seen in Figure 1.3(a) [8]. This device uses reflective beam steering to create coupling between input and output fibers in a fiber optic connection. The mirrors are torsionally actuated (tilted) by electrostatic attraction caused by electrodes under the mirror surface. The space etched between the mirrored surface and the underlying electrode layer and the configuration of the mirror hinge determine the maximum angle of deflection. Each mirror, or pixel, is individually controlled by its location, or address, on a MOS chip making the array a highly tunable device. Texas Instruments has developed and marketed a MEMS display technology known as a DMD, or

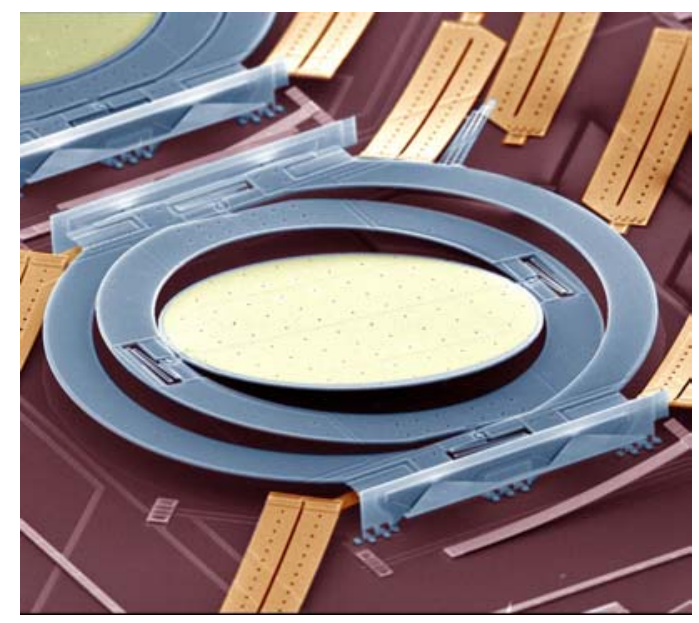

(a)

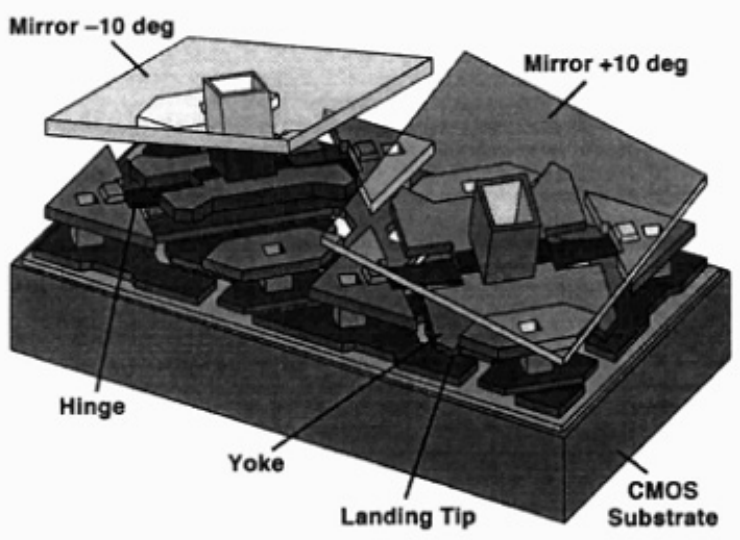

(b)

Figure 1.3: Torsional actuation MEMS devices: the WaveStar Lambda Router (a) (Lucent Technologies) [8], and two unit cells of the DMD projection display device (b) (Texas Instruments) [9]. 
digital micromirror device (Figure 1.3(b)) [9]. In an array, these devices form a display device that allows high fidelity data transmission, storage, and playback, as well as limitless reproduction without degradation. Display resolution is determined by the size and density of mirrors in the array. Single device display applications have also been developed [10].

Many free-space optical systems, such as astronomical telescopes and line-of-sight optical communications, experience irregularities that affect the received signal. These irregularities, or aberrations, are usually caused by free space turbulence, such as thermal distortion. Aberrations cause a fuzziness of the received signal, creating a level of uncertainty that could cause major problems in high precision applications. By coating the surface of the image or receiving plane with an array of integrated deformable mirrors, these aberrations can be reduced. Arrays of devices such as these have been tested and characterized by various research groups [11], [12].

\section{Gear/Motor Rotary Motion MEMS}

Another type of MEMS structure includes those that rotate in the plane of fabrication. Rotation can be achieved by varying the voltage of the "stator" electrodes situated around rotor electrodes with the opposite voltage applied, as in Figure 1.4(a). This variation causes a repulsion or attraction, much like that exhibited by linear comb resonators, except in a rotational direction. Like their trorsional counterparts, this type of MEMS has been explored for use in optical switching and planar scanning. An electrostatic polysilicon micromotor can be fabricated with a diffraction grating, consisting of two different spatial periods, on the surface of the rotor. This device can be used to redirect light at two different angles [1].Another method of achieving rotational motion is to combine gears with translational MEMS, such as the Sandia Microengine [13]. One lateral resonator device seen in Figure 1.4(b) operates out of phase with the second to cause a circular action of a geared device connected by polysilicon beams to both resonators. This gear can then be used to drive larger gears, geared racks, and larger areas of micromachined silicon in complex micromechanical systems (Figure 1.4(c)). These devices have been developed as safing and locking mechanisms for the arming systems of nuclear weapons. These which exhibit continuous motion and experience time varying operational parameters, such as wear and applied load, 
need a reliable system of microstructure monitoring to increase their reliability in safety critical systems.

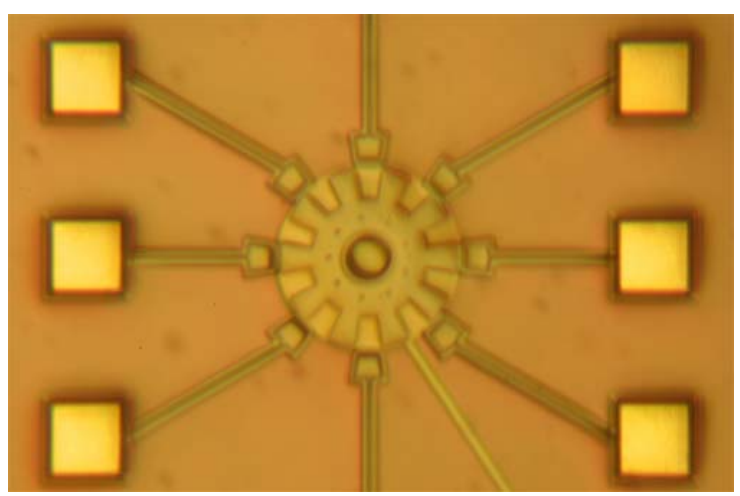

(a)

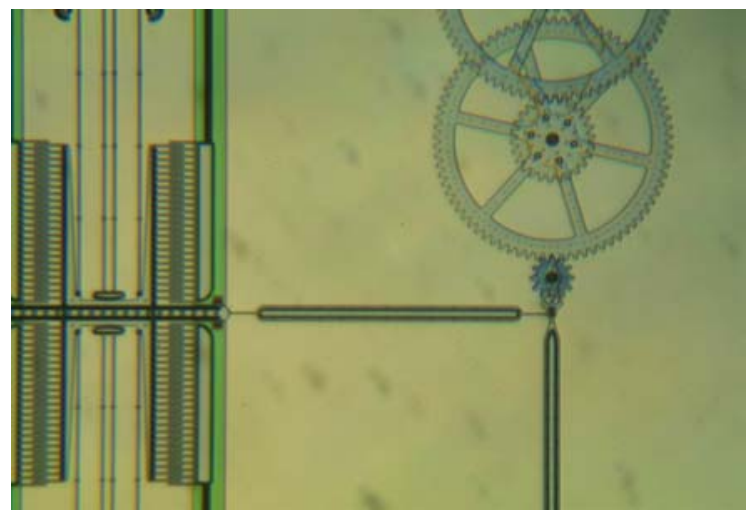

(b)

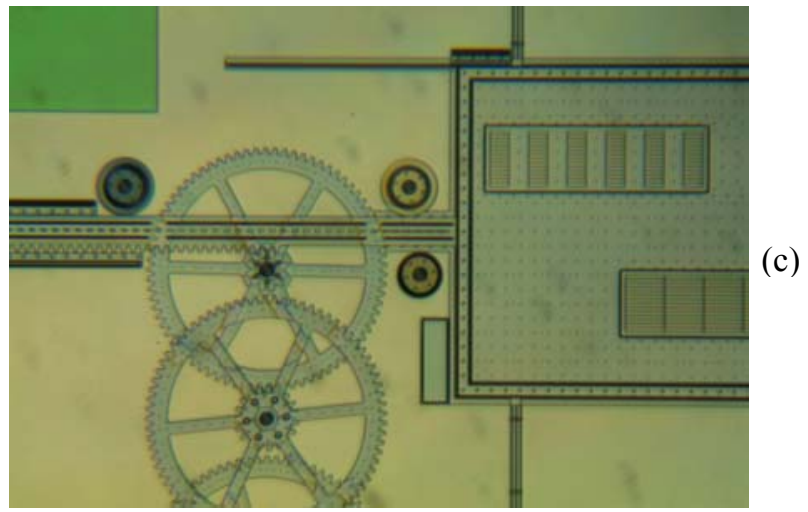

Figure 1.4: Rotational actuation MEMS devices: a MUMPs fabricated salient-pole side-drive micromotor (a) and the Sandia Microengine drive gear (b) and larger gears and microstructures driven by it (c).

\subsubsection{Modeling and Design}

Before a device is considered for fabrication, a thorough evaluation of the operation of the device must be performed to ensure proper device function for both sensors and actuators. The expanding market for MEMS has led to the rise of CAD tools that are not only used for designing device layouts, but also to simulate fabrication and operation of the device as well. IntelliSense's IntelliSuite and MEMSCAP's MEMS Pro are two of the major CAD tools of this type. They use finite element, boundary element, and structured block mesh analyses to develop a behavioral model for the drawn device. These tools often model not only mechanical operation, but examine electrical, thermal, and microfluidic effects as well.

Outside of the commercial CAD market, other approaches to MEMS modeling have been explored. Circuit-level methodologies have been proposed for design and simulation of 
MEMS by transforming the device into a schematic that can be behaviorally examined [14], [15]. Behavioral modeling using high-level functional descriptions coupled with statistical analysis has been performed as well [16], [17]. Detailed mechanical modeling of a variety of MEMS device elements and parameters, from resonator flexures to microengine linkage joints, has been performed to better understand device operation [18], [19]. After sufficient modeling of the MEMS has been performed, fabrication can be completed and characterization of the devices can be performed to determine how they will function under a variety of operating conditions.

\subsubsection{MEMS Characterization}

The small size of MEMS devices poses many challenges to characterizing devices under operation. The optimum conditions that cause the device to operate properly in the application it was designed for need to be determined. This often involves measuring parameters such as resonant frequency and range and direction of motion. Most devices are packaged in hermetically sealed chip packages due to the sensitivity of microactuators to certain atmospheric conditions, mainly humidity. Many unpackaged devices are examined in vacuum chambers to simulate actual operating conditions. To observe the device in motion, stroboscopic techniques and laser interferometry methods have been used to evaluate the movements of MEMS [20], [21]. Laser Doppler vibrometers have been developed that can measure both in-plane and out-of-plane motion of a variety of MEMS devices using similar optical techniques. Electrical means of sensing device movement, including capacitance measurement and MEMS circuit simulation, have been explored as well [15], [22], [23]. High-speed video imaging has also been utilized. One method uses bright-field optical microscopy and interferometric imaging to measure in and out-of-plane motion [24]. Another video characterization method uses Moiré patterns for ultrafine motion detection [25]. Videobased methods have also been combined with electrical measurements for validation of the different types of characterization efforts [26]. 


\subsubsection{Reliability and Failure}

One major area directly related to device characterization is device reliability. Knowing the conditions that can lead to MEMS device malfunction or failure before it is implemented allows device manufacturers to tailor their devices toward specific applications and can relate the difference in lifetime of the device operating under optimal and sub-optimal conditions. MEMS reliability is often divided into four main areas. These are materials reliability, structural reliability, process reliability, and packaging reliability [27]. Device malfunction and failure can occur due to faults occurring separately or simultaneously in one or more of these areas.

\section{MEMS Materials}

Materials reliability involves the quality of the materials that the MEMS is comprised of. Most MEMS are composed of thin films of single crystal silicon, polysilicon, silicon nitride, and/or silicon dioxide on silicon or glass substrates. Other materials, such as polymer, ceramic, and diamond thin films, have been utilized as well. Silicon MEMS processing has been studied thoroughly and optimized to minimize defects and impurities in the material layers used in device fabrication. However, degradation of the mechanical properties of the material can occur as the device ages, causing device performance degradation. This degradation often occurs as a result of the environment in which the device is operated. Oxide growth has been shown to increase the stiffness of silicon-based devices [28]. This can lead to a change in the resonant frequency of resonator-type devices. Oxide growth is also believed to hamper the performance of MEMS side-drive motors as well [29]. Delamination

of the layers making up a MEMS device can occur due to high residual stress between layers. The seams between these layers can be adversely affected by physical and chemical degradation as the device operates, causing a shift in device performance and even failure [27].

\section{MEMS Structures}

Structural reliability is an aspect of device geometry. Under constant operation, areas of localized stress become the center of fatigue and can lead to fractures. Proper modeling and design to strengthen or eliminate these high-stress areas can reduce this problem. Improper 
device design can lead to unwanted physical contact between areas of polysilicon with drastically different voltage polarities, causing electrical shorting and sometimes resulting in catastrophic failure. Stiction is perhaps the largest MEMS structural reliability issue. Moisture remaining in areas between moving structures after the removal of sacrificial oxide layers by wet etching processes causes the released parts to be held in place due to surface tension. Procedures such as Chronos Integrated Microsystems' dry $\mathrm{CO}_{2}$ release process help to alleviate these effects. Another major structural reliability issue is friction and the resulting mechanical wear that is present in systems where the MEMS elements are in constant moving contact. Accumulating wear debris can lead to performance degradation over time and the eventual seizure of moving parts. Anti-wear coatings, including chemical vapor deposited tungsten, have been explored to reduce frictional effects [30]. Ambient operating temperature can lead to structural reliability issues as well. The DMD device discussed earlier experiences an effect referred to as hinge memory, a residual mirror tilt remaining with no voltage applied, as a result of operation in high temperature environments [31]. If the operational drift due to temperature is consistent and repeatable, compensating electronics can be included to overcome its effects.

\section{MEMS Processing and Packaging}

As with any other microelectronic technology, MEMS process reliability is an important factor. Bulk micromachining has been improved to provide high throughput and yield, as have other processing methods such as surface micromachining, high-aspect ratio etching and LIGA techniques, and dissolved wafer processes [27]. New CAD tools that simulate fabrication processes and the rise of MEMS foundry services allow for rapid prototyping of device designs to determine the success of the fabrication procedures. Packaging reliability is a major factor in MEMS performance as well. Die attachment and wire bonding processes must not thermally affect the devices fabricated on the chip. For MEMS sensors, isolation of elements from unwanted environmental factors through total or partial hermetic sealing is crucial. Proper design and testing of packaging processes can reduce or eliminate the likelihood of MEMS failure due to package failure. 


\subsection{Integrated Optical Monitoring and Control Exploration}

Due to the small range of movement exhibited by MEMS devices, continuous lifetime monitoring of motion for control and failure analysis purposes poses difficulties. As discussed earlier, many MEMS monitoring schemes use bulk optical methods that provide low noise and high accuracy metrology. However, these are employed only during die-level testing prior to device packaging [32]. Electrical means of sensing device motion during device operation have been explored. However, the dynamic range of the signal associated with device movement is small compared to the drive voltage signal. As a result, the signal may be lost in the noise created by the higher voltages used to power the device [33]. Capacitive sensing circuitry may also be affected by static charge accumulating oxide surfaces present on MEMS [34]. An ideal solution is intra-package integrated optical microstructure probing. This would enable decoupled position monitoring for control and failure assessment and management over the lifetime of the system [5]. This type of monitoring is best suited for systems where optical signals are not an intrinsic part of device function.

This integrated optical monitoring (IOM) solution involves the use of integrated optical interconnects, an area that has been thoroughly studied and has long been known to offer significant benefits in the implementation of multicomputer interconnection networks. These benefits include added dimensionality, high bandwidth, and complex packaging [35]. MEMS optical monitoring schemes using bulk optical counter parts of integrated optical components such as waveguides and free-space optical interconnects have been shown, in preliminary testing, to provide an adequate means of optically monitoring MEMS device motion [36], [37]. While promising improved performance, the integrated optical monitoring elements must be compatible with current MEMS and other chip-level device and packaging technologies to minimize system complexity and costs.

\subsubsection{Preliminary Through-Wafer Monitoring Results}

Through-wafer optical monitoring has been experimentally shown to be effective in providing positional information of the MEMS lateral comb resonator pictured in Figure 1.5(a) [38]. Results to date have been achieved using fiber optics and GRIN and bulk optical lenses to deliver and collect a through-wafer infrared probe beam interacting with the MEMS 
device. Figure 1.5(b) shows a preliminary image of the MEMS device and through-wafer optical probe supplied by a $1310 \mathrm{~nm}, 50 \mu \mathrm{m}$ fiber-coupled LED, which is focused to approximately $40 \mu \mathrm{m}$ by a single GRIN lens. The $1310 \mathrm{~nm}$ wavelength was chosen because of the transparency of silicon to light IR wavelengths. Using a double GRIN lens configuration as shown in Figure 1.5(c), a $9 \mu \mathrm{m}$ core pigtailed laser diode source output operating at $1310 \mathrm{~nm}( \pm 1 \mathrm{~nm})$ can be focused to a spot size of approximately $15 \mu \mathrm{m}$ diameter.

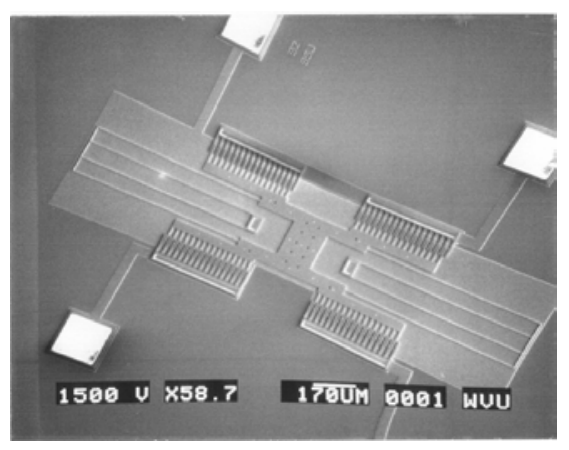

(a)

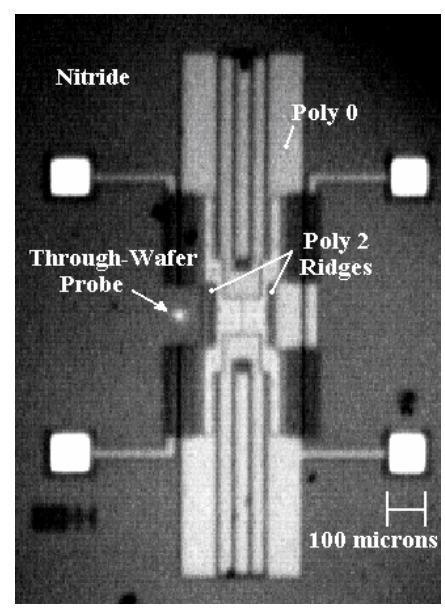

(c)

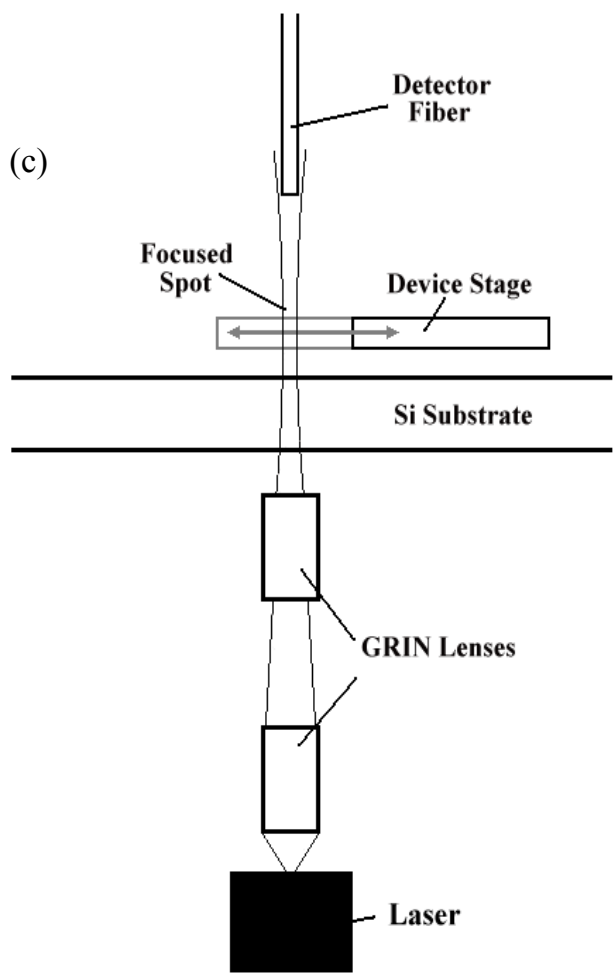

Figure 1.5: MUMPs fabricated lateral comb resonator (a). The initial through-wafer bulk optical monitoring scheme (b). IR image of focused through-wafer spot (c).

The interaction of the probe beam by the moving device causes an intensity change in the optical intensity. This change in intensity is picked up by the detector fiber coupled to a photoreceiver. 


\section{Stationary Device Through-Wafer Intensity Examination}

After the optical probe beam was established, the transmission intensity of the beam through the layers of polysilicon comprising the resonator translation stage was measured. This was achieved by moving the probe optics while keeping the MEMS die in a fixed position and the device stationary (unpowered). Figure 1.6 shows the area scanned and its cross-section, as well as the results of scanning with both single ( $8 \mu \mathrm{m}$ core) and multimode (47 $\mu \mathrm{m}$ core) detector fibers connected to an InGaAs photoreceiver.

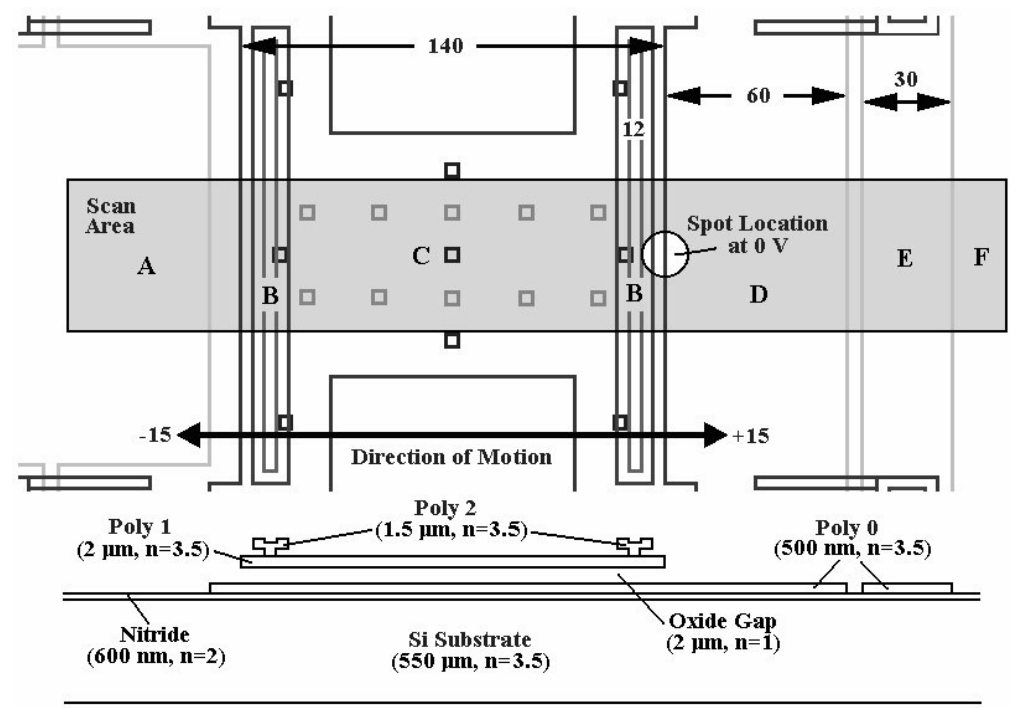

(a)

Through-Wafer Power (LD, $47 \mu \mathrm{m}$ core detector)

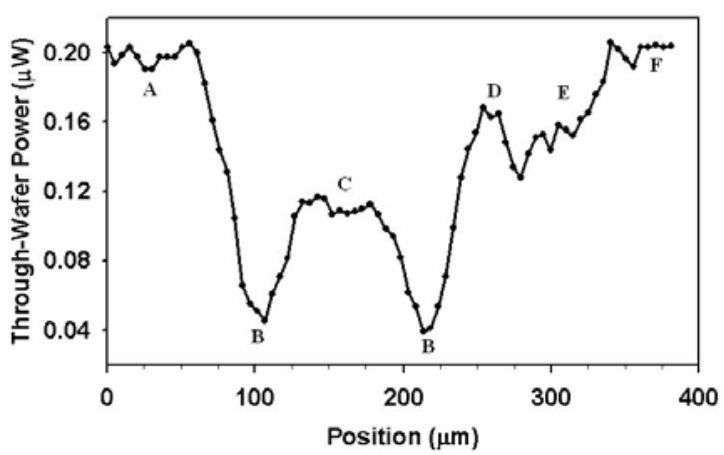

(b)
Through-Wafer Power (LD, 8 $8 \mathrm{~m}$ core detector)

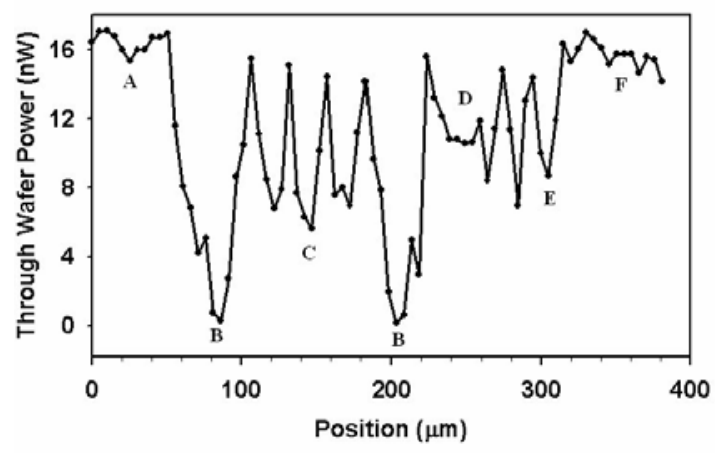

(c)

Figure 1.6: Through-wafer scan of a stationary lateral comb resonator stage. (a) Scan area, cross section view for thickness illustration, and spot location at $0 \mathrm{~V}$ for probing a powered device (direction of motion indicated). (b) Scan data with $47 \mu \mathrm{m}$ detector fiber and (c) $8 \mu \mathrm{m}$ fiber. 
The data obtained from the scan using the multimode fiber detection system shows a distinct change in through-wafer beam intensity as the beam passes through different thicknesses of polysilicon, with letters A-F corresponding to scan areas A-F in Figure 1.6(a). Layer names are defined by Chronos Integrated Microsystem's Multi-User MEMS Processing Service (MUMPS). The $12 \mu \mathrm{m}$ wide regions of Poly 2 on the edges of the stage appear as distinct minima in the scan, (labeled B). Area $\mathrm{C}$ represents transmission through the main bulk of the stage comprised of Poly 1 . The gradual transition of intensity in the scan data is due to the large aperture of the detector fiber, an effect confirmed by simulated convolution of the probe beam and device features. Table 1.1 expresses the differences in through-wafer intensity from region to region in terms of percentage transmission (measured far from feature edges that induce diffraction) that have been normalized with respect to transmission through featureless regions. These regions gave a baseline signal loss of $50 \%$ after passing through the backside polished, $550 \mu \mathrm{m}$ thick die substrate and device-side nitride layer. This baseline loss can be reduced to $27 \%$ by the addition of a $491 \mathrm{~nm}$ antireflection layer on the polished die back. Theoretical values determined from evaluating the Jones matrix for the multilayer stack transmission path (neglecting film roughness) are included for comparison. These results show that an $80 \%$ dynamic range of the throughwafer probe signal is achievable. The addition of the aforementioned antireflection layer, as well as an adjustment of the thickness of the device-side nitride film, should result in 99\% transmission at $1310 \mathrm{~nm}$ (neglecting reflection losses).

Table 1.1: Percentage Transmission of MUMPs MEMS films at 1310nm.

\begin{tabular}{|c|c|c|}
\hline $\begin{array}{c}\text { Feature } \\
\text { (MUMPs layers) }\end{array}$ & Calculated (\%) & Measured (\%) \\
\hline \hline Poly 0 & 74 & $60-75$ \\
\hline Poly 0 - air- Poly1 & 70 & $50-60$ \\
\hline Poly 0 - air - Poly 1- Poly 2 & 21 & 20 \\
\hline
\end{tabular}

The single mode fiber scan illustrated in Figure 1.6(c) shows more clearly the diffraction effects caused by device feature edges. These effects include an apparent enhancement of the dynamic range of the signal near these features. The equidistant maxim and minima in scan area $\mathrm{C}$ are believed to arise from etch hole and poly 1 dimple features in the device stage. 


\section{Through-Wafer Observation of Device Motion}

After the dynamic range of the through-wafer signal was determined to be suitable, probing of the device under powered conditions was performed. A schematic representation of the InGaAs photoreceiver input/output relationship is given in Figure 1.7, indicating a transimpedance gain of $1 \times 10^{7} \mathrm{~V} / \mathrm{A}$.

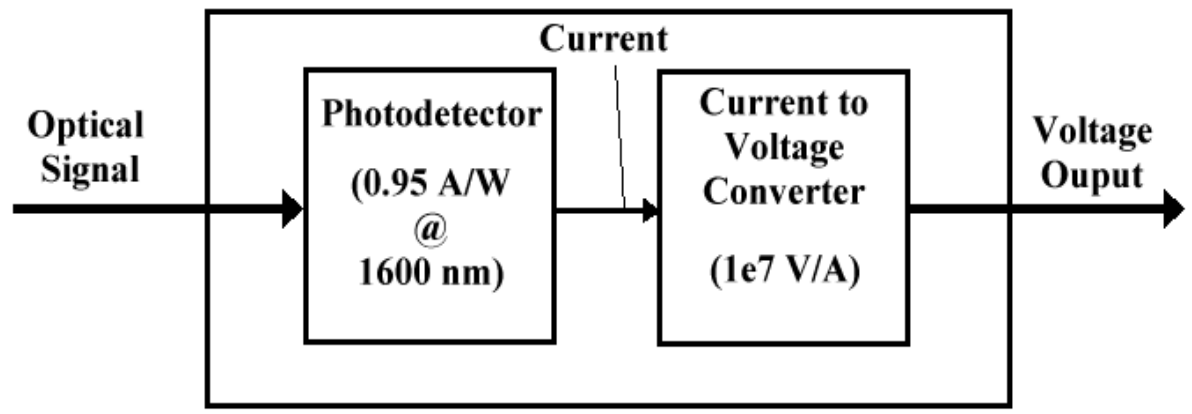

Figure 1.7: InGaAs photoreceiver input/output relationship.

The effects of static deflection of the device stage on the probe beam were analyzed by holding the drive stators at $\pm 17 \mathrm{~V}$ dc while varying the stage voltage between $-15 \mathrm{~V}$ and +15 V. This gave the through-wafer transmission optical signal variation in Figure 1.8, plotted with static stage deflection calculated as a function of applied stage voltage. The position of the probe spot with the stage at $0 \mathrm{~V}$ is illustrated in Figure 1.6(a), along with the direction of motion exhibited by the stage with applied voltage polarity.

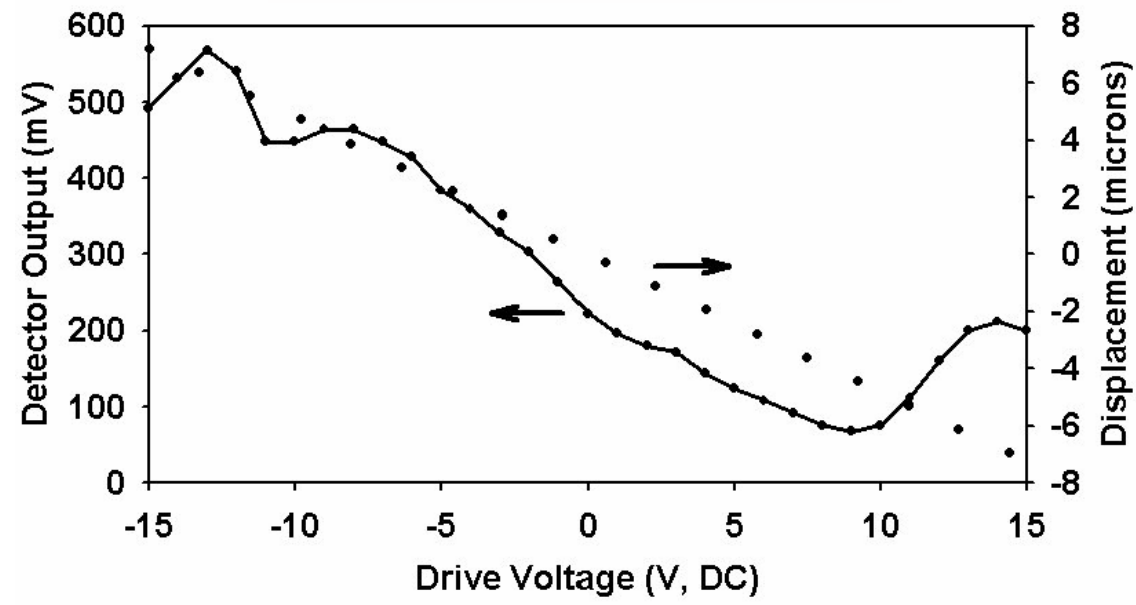

Figure 1.8: Through-wafer optical signal in volts and calculated static deflection versus applied voltage 
The increase in the detector signal as the drive voltage approaches $-15 \mathrm{~V}$ is a result of the stage moving completely out of the path of the probe beam. The decrease in detector signal as the drive voltage approaches $+10 \mathrm{~V}$ is a result of the beam being interrupted by Poly 2 structures on the device stage. The increase in detector signal after $+10 \mathrm{~V}$ is believed to be a result of the Ploy 2 structure passing completely through the probe path, leaving only Poly 1 stage areas. These results indicated a positional sensitivity of $40 \mathrm{mV} / \mu \mathrm{m}$, or $10 \%$ modulation per micron.

Dynamic deflection analysis was performed by applying $\pm 10 \mathrm{~V}$ dc to the stator combs and a $10 \mathrm{~V}(20 \mathrm{~V} \mathrm{p}-\mathrm{p})$ sinusoidal signal to the translation stage. Again, the beam waist was positioned in the location indicated in Figure 1.6(a). Figure 1.9 shows detector output, normalized displacement, and input drive voltage waveforms for drive voltage frequencies of $0.5,1.8$, and $2.2 \mathrm{kHz}$.

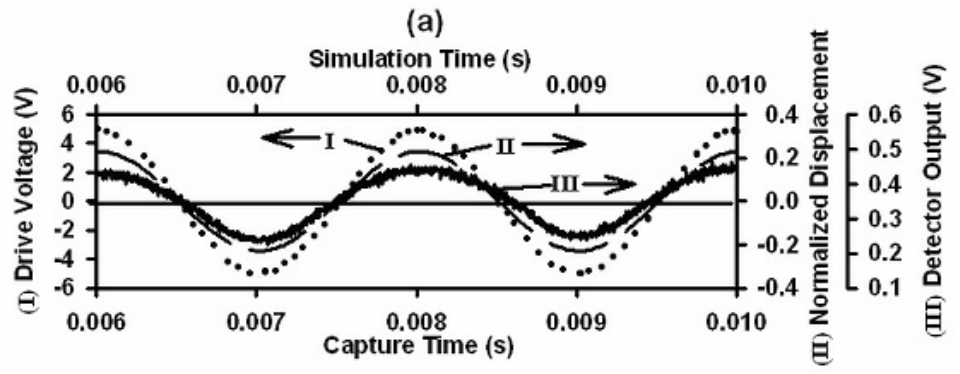

(b)

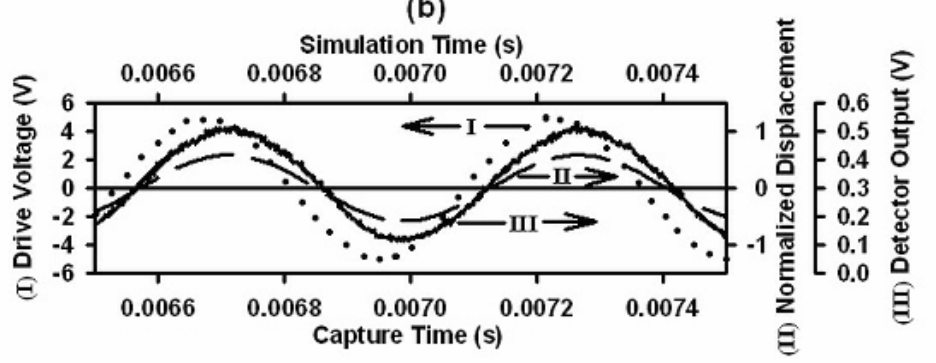

(c)

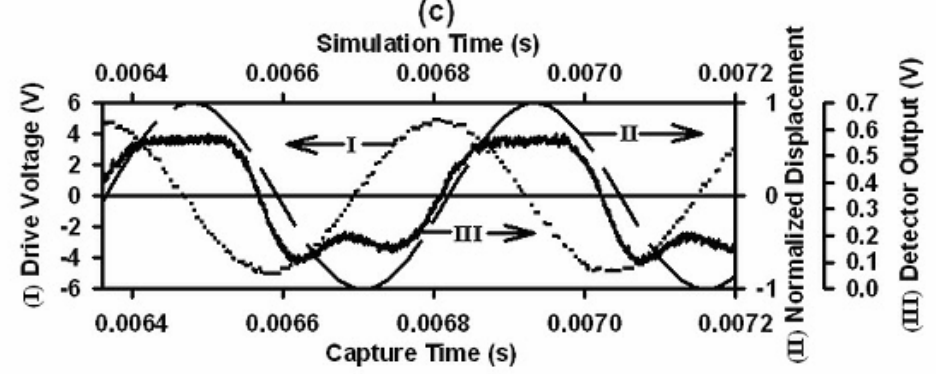

Figure 1.9: Drive voltage (I), normalized stage position (II), and throughwafer detector voltage (III) versus time for (a) 0.5 , (b) 1.8 , and (c) $2.2 \mathrm{kHz}$. 
Displacement was approximated by using the standard model of a damped forced harmonic oscillator for the lateral comb resonator device [39]. The air damping coefficient and spring constant were calculated using the mass and geometry of the specific device [18], [40], [41]. The resonant frequency of the $400 \mu \mathrm{m}$ flexure device tested was calculated to be $2.79 \mathrm{kHz}$. A stage mass of $0.245 \mu \mathrm{g}$ was determined from as-drawn mask geometries and MUMPs layer thicknesses. Due to the MUMPs process linewidth variation of $\pm 0.3 \mu \mathrm{m}$, the resonant frequency should fall between 2.19 and $3.44 \mathrm{kHz}$.

Figure 1.9 shows that, as the frequency of the input voltage is increased, the peak-to-peak voltage of optical signal increases while moving out of phase with the MEMS drive voltage. This follows the behavior of the calculated displacement waveform and agrees with the behavior of a forced harmonic resonator with damping. At $2.2 \mathrm{kHz}$, the input voltage is nearly 90 degrees out of phase with the detector signal. The shape of the waveform at this frequency is a result of a large displacement of the stage causing it to remain out of the path of the probe beam at one extreme range of motion, resulting in flat areas in the maximum cycle of the signal. At the opposite extreme range of motion, the stage moves so that the beam passes completely under the poly 2 structures on the stage, resulting in the structure observed at the minimum cycle of the signal.

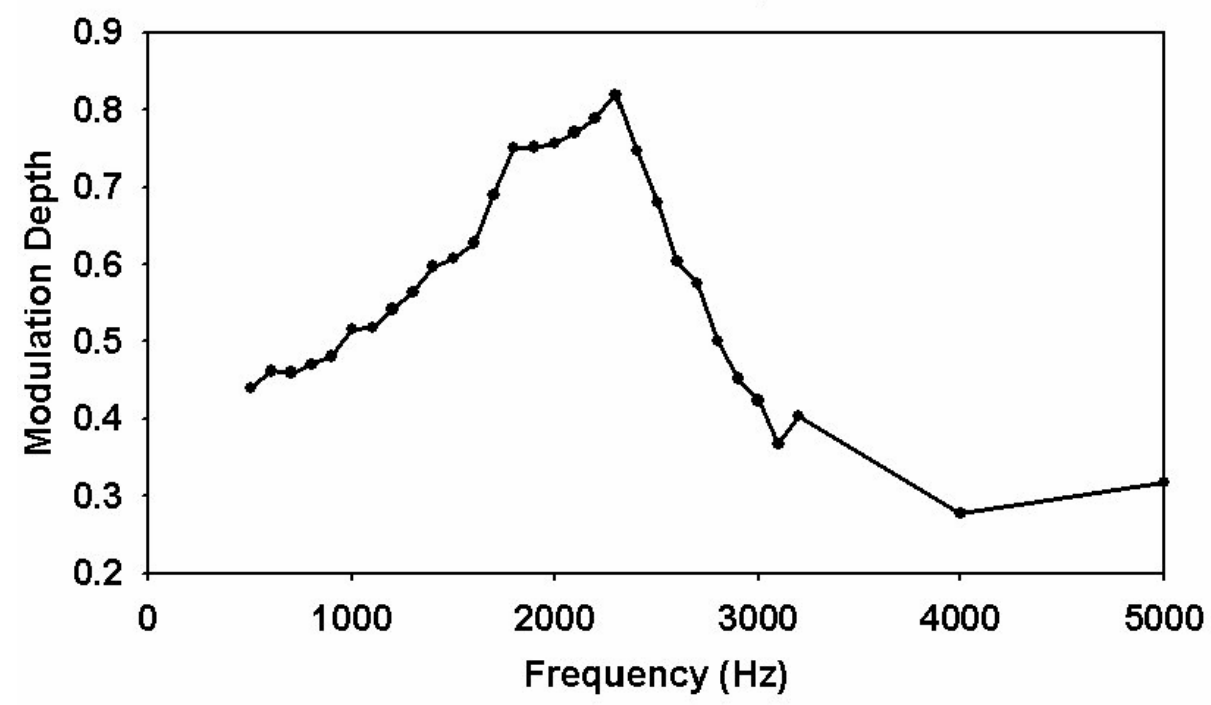

Figure 1.10: Through-wafer optical signal modulation depth versus frequency.

Figure 1.10 illustrates the frequency dependence of the amplitude of the through-wafer optical signal. This data is expressed as the modulation depth of the motion signal, defined as 
the change in measured detector output voltage at a given frequency relative to the voltage output at $0 \mathrm{~V}$ drive voltage normalized by the output signal voltage at $0 \mathrm{~V}$ drive. The resonant frequency of the device can be clearly observed around $2.2 \mathrm{kHz}$, a value that is within the theoretical range presented earlier. At this frequency, the maximum displacement of the device takes place, causing the largest modulation depth, following the expected behavior of a forced harmonic oscillator. The dip in the plot near the resonance peak is most likely a result of the attenuation of the signal due to the Poly 2 ridge on the stage of the device, causing an effect similar to the waveform illustrated in Figure 1.9(c).

These tests have shown that through-wafer optical probing methods are suitable for characterizing and measuring the behavior of lateral harmonic oscillators. However, for the application of control routines to be successful, certain problems inherent in the system need to be addressed. Vibrational instability is a major problem when trying to apply control routines. The optical probing system used in preliminary studies allows the optical signal to experience changes due to vibrations of the probe set-up, in addition to changes in device operation. This effect makes it difficult for the control routine to distinguish between changes in device behavior as a result of a controlled added disturbance versus unwanted table vibration. MEMS control also requires a detailed knowledge of the positional state of the device at all times. A precise knowledge of the local features on a device plus knowledge of the limits of range of motion are required in order to correlate the optical output signal to the actual displacement of a MEMS element.

This research focuses on overcoming these problems. The use of integrated Fresnel zone plate lenses is studied as a means to overcome vibrational instability by moving toward true integrated through-wafer optical monitoring. The optical microprobe assembly itself has been mechanically reinforced for preliminary closed-loop control studies using the bulk optical through-wafer probe to improve stability and to achieve a high signal-to-noise ratio. New device features have been studied for more accurate determination of the MEMS positional state. Grating structures that have been proposed as a means of determining absolute device position have been implemented [42]. These improvements have allowed for successful application of closed-loop control routines. 


\subsubsection{Through-Wafer Optical Probe System Redesign}

Integrated optical elements are necessary to completely eliminate the vibrational sensitivity of the input and detector elements in the through-wafer probing setup. As the next step toward fully integrated optical monitoring, the multiple-GRIN lens setup used to focus the spot in the plane of the moving stage of the device has been replaced with a quartz substrate with Fresnel zone plate lenses fabricated on its top and bottom surfaces. These lenses has been used to direct and focus the spot in the same manner as the multiple bulk optical arrangement.

\section{Integrated Optical Monitoring Loss Analysis}

Before integrated optical monitoring is applied to MEMS for through-wafer optical monitoring and feedback control, it is wise to examine such a system to determine if factors such as optical signal power loss and degradation will be significant enough to warrant the development of another method to obtain the positional state of MEMS devices. Figure 1.11 shows a schematic view of the integrated optical probe configuration similar to the one being implemented in the current phase of this research. Letters A-G notes different areas of possible reflection loss, absorption, or non-ideal diffraction efficiency.

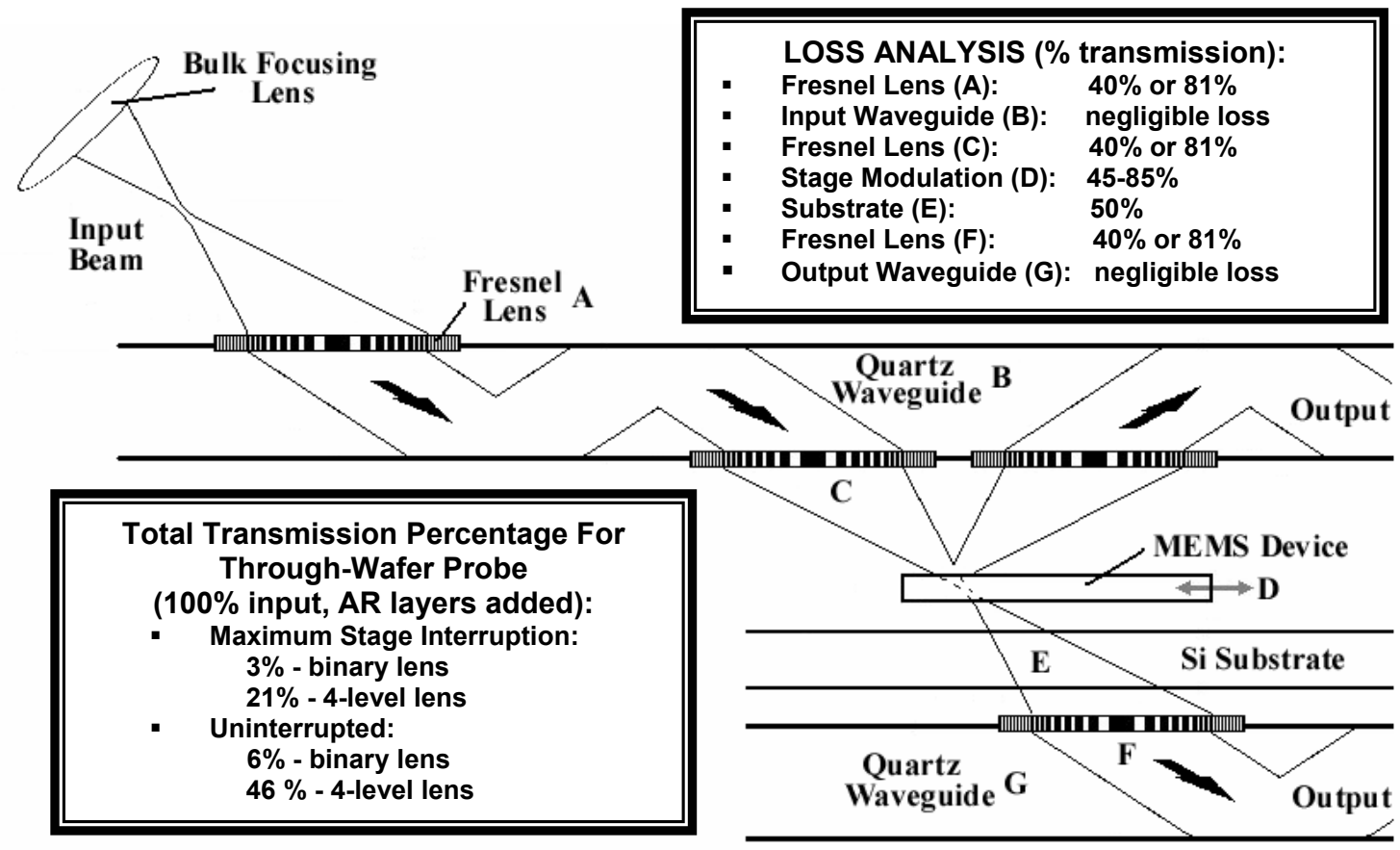

Figure 1.11: Illustration and loss analysis of proposed IOM scheme. 
In the experimental setup, a bulk optical lens will be used to couple a collimated input beam to the quartz waveguide through a Fresnel zone plate lens (ZPL). For the analysis of the IOM architecture shown in Figure 1.11, both binary (40\% efficiency) and 4-level ZPLs (81\% efficient) were considered [43]. The table of losses the total transmission intensity experiences assumes unity or $100 \%$ power input to the bulk optical lens. The first coupling lens will cause will cause a power loss of 40 and $81 \%$, for binary and 4-level phase optics, respectively. For a totally internally reflected propagation path, loss in the quartz waveguide will be negligible. The next coupling lens used to focus the probe beam in the device plane will further reduce the intensity by 40 and $81 \%$. Interruption of the beam by the moving stage of the device has been shown to cause between $45-85 \%$ decrease in transmission intensity. The transmission loss through the substrate was measured to be $50 \%$ in featureless areas without nitride AR layers, but can be lowered to $10 \%$ with such layers on the MEMS die front and back [44]. The third lens, collimating and coupling the beam into the output waveguide, decreases intensity by another 40 and $81 \%$. This path gives the values shown in Figure 1.11. With maximum stage interruption, the output intensity will be $1 \%$ of the input for a binary lens and $12 \%$ for a 4 -level lens. The uninterrupted beam will be 3 and $21 \%$ of the original input power. With nitride AR coating of the MEMS die, these values become 3 and $21 \%$ with maximum stage modulation and 6 and $46 \%$ uninterrupted. In all cases, a signal modulation of greater than $50 \%$ is obtained. An IOM scheme using reflection from the microstructure plane would eliminate the loss in the substrate, but would add to the complexity of system alignment

\subsubsection{Integrated Fresnel Zone Plate Lenses}

\section{Fresnel Lens Design and Fabrication}

Even though four-level phase lenses (two mask levels) allow increased coupling efficiency, binary lens elements have been chosen for the initial design validation studies of this research due to their relative ease of fabrication. Binary zone plate lenses are one of the simplest type of diffractive elements in design and operation, but have some limitations in practical implementation. As shown in the previous section, diffraction efficiency is limited to less than $50 \%$, and off-axis binary lenses will most likely result in comatic aberrations in 
the output beam intensity pattern. Binary lenses provide a good starting point at which to test key design, processing, and testing issues involved in determining the feasibility of using diffractive optical microelements in this system.

Off-axis lenses have been used at the input and output of each guide plane with small input/output angle difference. This design relaxes the otherwise severe lithography requirements arising from deep submicron repetitive features resulting from the large angle designs. The Karl Suss M6 mask aligner available can readily resolve linewidths down to $0.75 \mu \mathrm{m}$. From a practical standpoint, this choice also enables viewing of the probe spot on the MEMS substrate during alignment and experimental evaluation. A binary lens design of this type is shown in Figure 1.12

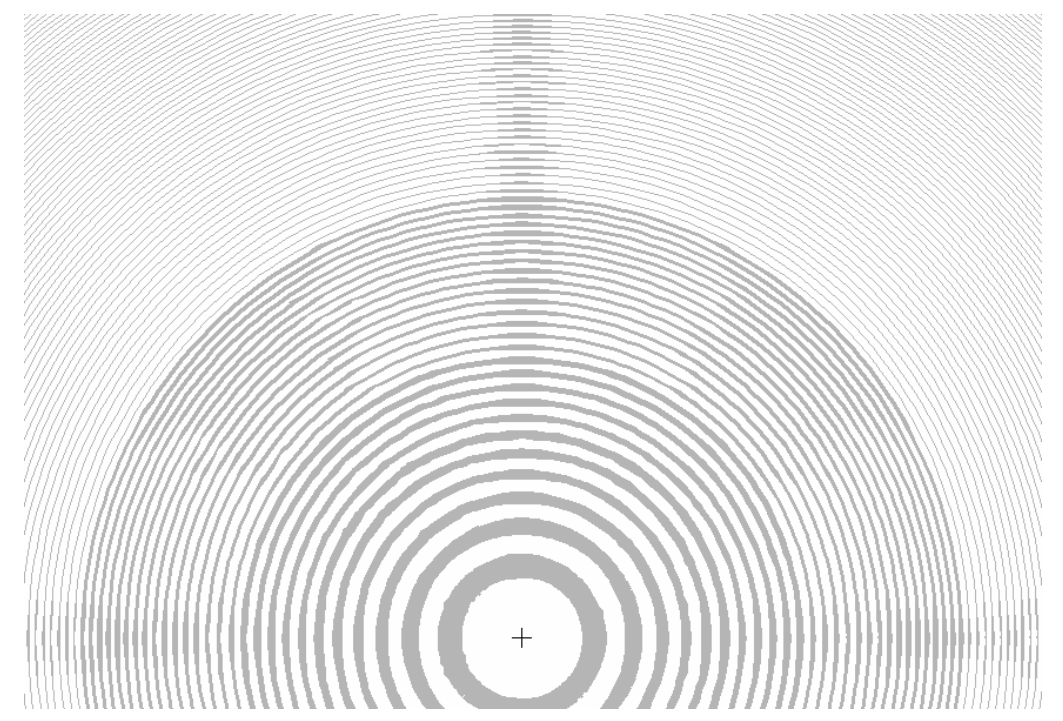

Figure 1.12: Representation of a $10^{\circ}$ off-axis binary ZPL.

Designed for $1310 \mathrm{~nm}$, this lens can bend and focus an off-axis, divergent input beam 10 degrees beyond the input angle for collimated total internal propagation within the waveguide substrate With an input angle of $41.8^{\circ}$ (the critical angle for a quartz waveguide in air), this gives the propagating beam an angle of $\sim 62^{\circ}$. Feature sizes in this $1 \mathrm{~mm} \times 1 \mathrm{~mm}$ area lens are no smaller than 1.75 microns, well within the limits of the mask alignment system available. Larger output angle lenses yield features too small to be fabricated repeatedly and reliably. Lenses that accept an off-axis input and focus on-axis could be achieved if losses due to non-total internal reflection propagation are reduced by the addition of reflective metal 
cladding areas to the substrate surface. This would allow for on-axis inputs to be collimated and coupled into the waveguide, and similarly coupled out for probing MEMS structures. The binary lenses designed can be used for visible wavelengths with an increase in focal length. A visible helium-neon wavelength $(637 \mathrm{~nm})$ was used in the initial stages of the lens substrate's implementation to become accustomed to the alignment issues inherent in such a system and aid in the alignment process.

Initial zone plate lens fabrication was performed by reactive ion etching $\mathrm{SiO}_{2}$-coated 4inch silicon wafers using an Oxford Instruments Plasmalab 80+ system. These test wafers were used to determine the accuracy of the photolithography processing, the reactive ion etch parameters that give the most anisotropic features, and the etch rate of $\mathrm{SiO}_{2}$ under these conditions. After the photolithography and etching processes were refined and are readily repeatable, 3-inch fused silica substrates will be used. Lenses have been etched into both sides of the substrate.

\section{Implementation}

After the lenses were fabricated on a quartz substrate, testing was performed to determine the amount of transmission degradation, if any, which may occur as a result of the etching process, reducing the surface quality of unmasked areas of the substrate. This was done by passing light through areas of a test substrate that has both etched and unetched surfaces. This scheme is illustrated in Figure 1.13.

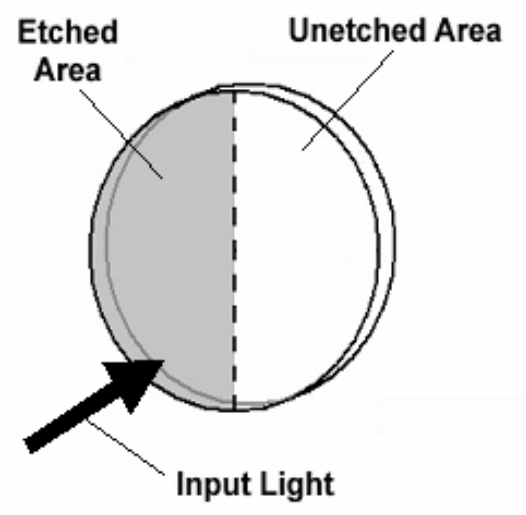

Figure 1.13: Transmittance testing setup. 
The transmitted intensity of collimated input light (at both $637 \mathrm{~nm}$ and $1310 \mathrm{~nm}$ ) was examined, and an evaluation of the different probing areas was made.

Before fabrication of the lenses and waveguide, the spot quality and focal length of the lenses was calculated to determine if suitable probe spots can be obtained. After this, the lens and waveguide substrate were used in the through-wafer optical probing apparatus, adding another level of complexity to the already intricate system. Optical sources and focusing optics for both visible and infrared probe beams had separate $x, y$, and $z$-axis adjustments that are coupled to the three-axis adjustment of the waveguide substrate. To simplify alignment and allow for spot observation, these elements were located above the device plane, as shown in Figure 1.11. As mentioned earlier, off-axis focus allowed for spot observation in the device plane. Characterization studies similar to those performed using the bulk optical lens arrangement were performed to determine the feasibility of employing the integrated optical substrate for through-wafer monitoring and control.

\subsubsection{Vibration Isolation}

Due to the added complexity that arises with the addition of the integrated optics plane, and in order for preliminary control efforts to be studied at the same time that the Fresnel lens fabrication is taking place, the optical probe setup was reconstructed using more stable elements. Motorized translation stages with small $(0.5 \mu \mathrm{m})$ incremental travel were used to prevent adjustment backlash, allowing stable probe spot positioning as well as fine adjustment of the probe location. While not completely vibration free, this new setup allowed the through-wafer optical probe to be used in the application of simple closed-loop control routines.

\subsubsection{Absolute Position Determination}

The control routines being considered for MEMS control in this research involved using microstructure position to estimate microstructure speed. Early research in through-wafer monitoring using a single-opening probing area (Figure 1.14) produced a large dynamic range of optical probe signal with small MEMS motion, but provided little information as to the actual displacement of the device stage. 


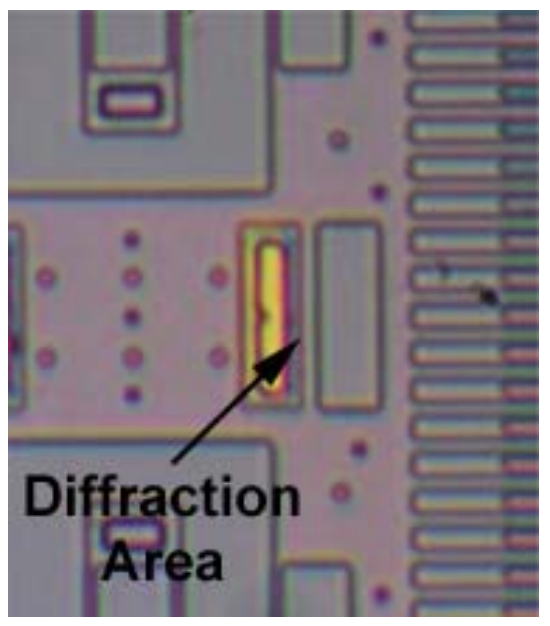

Figure 1.14: Single opening through-wafer probing MEMS device.

The range of the initial signal could indicate in-plane as well as out-of-plane motion, due to the levitation effects more likely to occur at maximum travel limits of the stage. This levitation changes the thickness of the air gap between the device planes, affecting the through-wafer intensity. The large range of the signal could correspond to anywhere from 2 to 10 microns of motion, depending on the frequency and amplitude on the input DC and AC drive voltages, since only one major change in intensity is present during one cycle of motion.

Using a diffraction grating fabricated on the moving stage of the device as the probing area could eliminate the guesswork involved in interpreting the signal from single-opening devices (Figure 1.15).

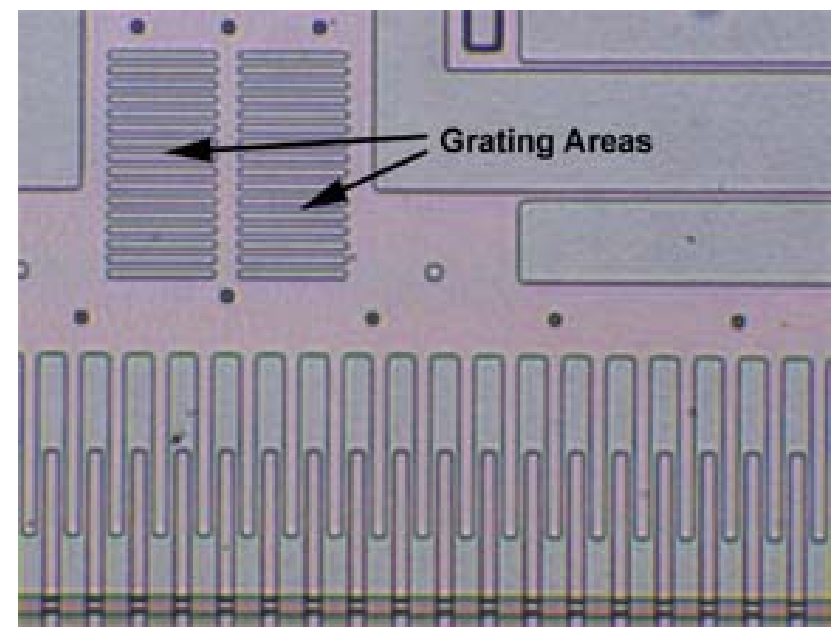

Figure 1.15: Grating structure through-wafer probing area. 
The acquisition of a laser Doppler vibrometer, to measure actual displacement of the stage, would provide a method of validating these theories. Another solution is theoretical modeling of the interaction of the probe beam with the device stage geometry. To study the through-wafer optical signal resolution of both the single-opening and grating stage geometries, optical field analyses were performed using lateral comb resonator model parameters (mass, damping, and spring constant) to explore through-wafer diffraction effects and probe beam intensity patterns. These analyses were carried out using Matlab programs. Theoretical and experimental optical output waveforms were examined to determine the positional resolution available, as well as the accuracy of the modeling program in predicting optical output behavior. After displacement is known, the successful application of nontrivial closed-loop control routines will be possible.

\subsubsection{Closed-Loop Control of MEMS}

The microstructure positional information signal obtained from the through-wafer optical probe has been used to experimentally validate microsystem mechanical models, determine model parameters, and apply closed-loop position control to the lateral comb resonator device using a real-time data acquisition and control system under various operating conditions [45], [46]. To date, standard linear techniques, including proportional-integral (PI) and proportional-integral-differential (PID) control, have been simulated and used experimentally with the mechanically reinforced through-wafer optical probing setup that reduces the effects of unwanted vibrations.

One method of improving the response time of the control routine is the utilization of a real time data acquisition board. This type of board often has a dedicated processor that performs calculations independent of the PC operating system. Using sliding mode tracking control instead of PID methods can also reduce controller response time. Sliding mode tracking control requires more knowledge of the positional state of the stage, making it difficult to apply this type of control to single-opening resonator stages. The grating structure stages discussed earlier show the potential of overcoming this limitation.

To determine the effectiveness of using the grating structure lateral comb resonators for MEMS control, the reinforced bulk optical through-wafer probing setup has been employed to explore control routines dependent on detailed position information. After fabrication, 
probe setup implementation, and signal characterization of the integrated zone plate lens substrate has been completed, through-wafer optical probe signals obtained from the arrangement outlined in Section 3.2.1 will be used for control purposes.

\subsection{Summary}

Successful through-wafer optical probing of MEMS for lifetime monitoring and control ultimately requires integrated optical components for mechanical stability of the probe beam to obtain an optical signal that provides detailed information about the position of the device during operation. This research has explores the fabrication and implementation of Fresnel zone plate lenses for probe beam delivery as the next step toward true integrated optical monitoring. It has also focused on the examination of grating structures fabricated on MEMS lateral comb resonator device stages as a means of determining absolute device position for control applications. In parallel with Fresnel lens fabrication, redesign of the through-wafer optical microprobe setup has allowed for the application of closed-loop control routines using the position information obtained from the grating structures. Figure 1.21 shows a flow of research tasks required to meet the final research goal.

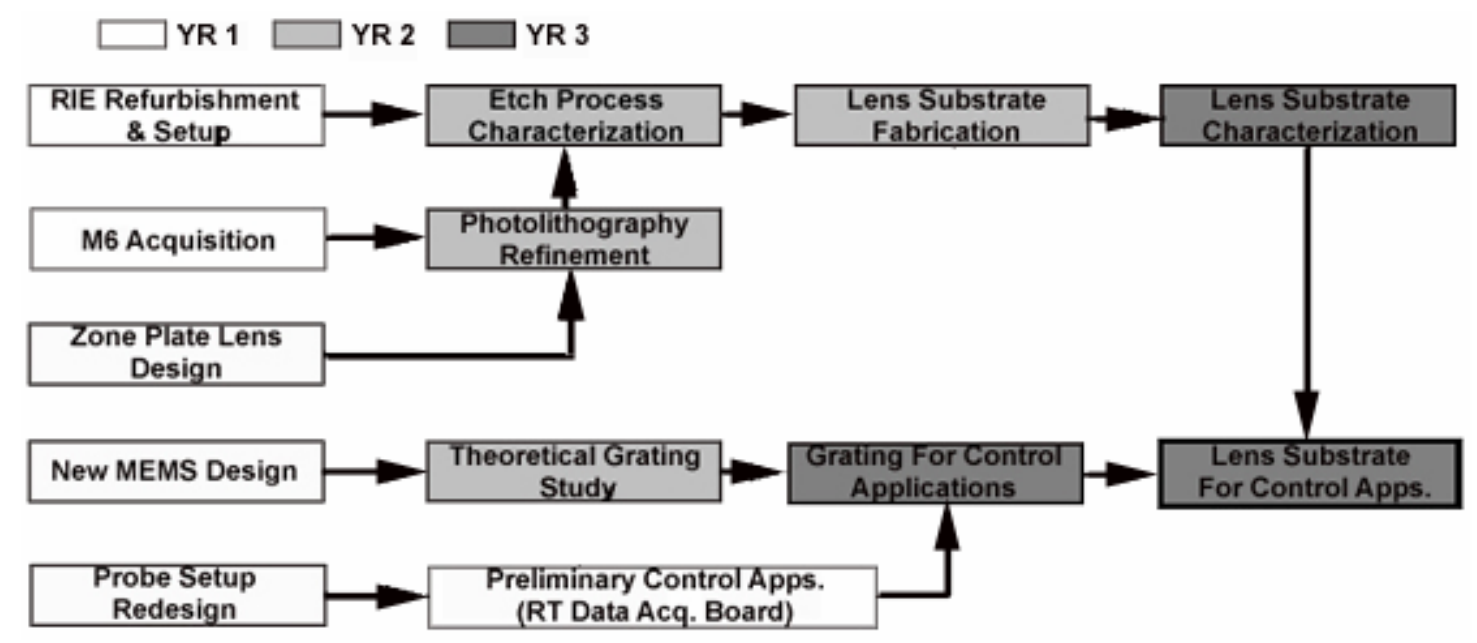

Figure 1.16: Research task flow illustration.

Chapter 2 presents the theory used for MEMS model development, Fresnel zone plate lens design, and optical analysis. Chapter 3 discusses changes in MEMS device and optical probe system design for improved through-wafer signal acquisition. Through-wafer diffraction studies using theoretical and experimental data are outlined in Chapter 4. Fresnel 
lens design, fabrication, and testing are covered in Chapter 5. Chapter 6 presents research conclusions and suggestions for future work. 


\section{Chapter 2 Theory}

The material presented in this chapter contains the theory that is the basis for understanding lateral comb resonator devices, diffraction effects of stage features, Fresnel lens design, and laser diode to fiber coupling. Section 2.1 deals with the development of a system model for the lateral comb resonator. To study the possibility of obtaining accurate position information from the optical signal, diffraction caused by the probing areas of the device stage was examined. Section 2.2 presents a study of diffraction progressing from simple plane wave interaction with an infinite slit to Gaussian beam diffraction through a transparent grating. Section 2.3 discusses the development of on and off-axis Fresnel diffraction patterns. Section 2.4 addresses the coupling efficiency in the fiber optic delivery and signal recovery system used in initial stages of this research.

\subsection{MEMS System Model Development}

In order for to control MEMS to the submicron level, an accurate system model must be developed. Previous examinations of comb resonator displacement were done using a system model that neglected the effect of the damping parameter, leading to unrealistic displacement values at the resonant frequency of the device. A more accurate device representation is that of a forced harmonic oscillator with damping. The first order differential equation representing this type of device is given as [39] 


$$
F_{e}=m \ddot{x}+\beta \dot{x}+k x+F_{d},
$$

where $x$ is the position of the stage, $m$ is the stage mass, $\beta$ is the damping coefficient, $K_{s}$ is the spring constant for one flexure, and $F_{d}$ is the load force (which is zero in this case). In this study, lateral translation of the stage is defined as being in the $x$-direction.

The force generated by the electrical input, $F_{e}$, is calculated using

$$
F_{e}=-4 n \varepsilon \frac{t}{g}\left(V_{b} \cdot V_{s}\right)
$$

where $n$ is the number of comb fingers, $\varepsilon$ is the permittivity of air, $t$ is the vertical thickness of the fingers, $g$ is the gap between stator and translation comb fingers, $V_{b}$ is the dc voltage supplied to the stator combs, and $V_{S}$ is the sinusoidal voltage signal applied to the translation stage. Accurate determination of these device parameters is essential for the development of a valid system model.

\section{Mass}

Mass can be approximated by multiplying the stage volume of the as-drawn geometry and the density of LPCVD deposited polysilicon $\left(2.33 \times 10^{-15} \mathrm{~kg} / \mu \mathrm{m}\right)$. A more accurate value can be obtained by calculating the effective mass of the stage in the $x$-direction, given by [47]

$$
m_{x}=m_{s}+\frac{1}{4} m_{t}+\frac{12}{35} m_{b}
$$

where $m_{s}$ is the mass of the stage, $m_{t}$ the mass of the flexure trusses, and $m_{b}$ is the mass of the flexure beams. Since the motion of the devices used for this research is predominantly lateral in nature, the $x$-component of the effective mass is most dominant. The dimensions used to calculate truss and beam mass are illustrated in Figure 2.1. 

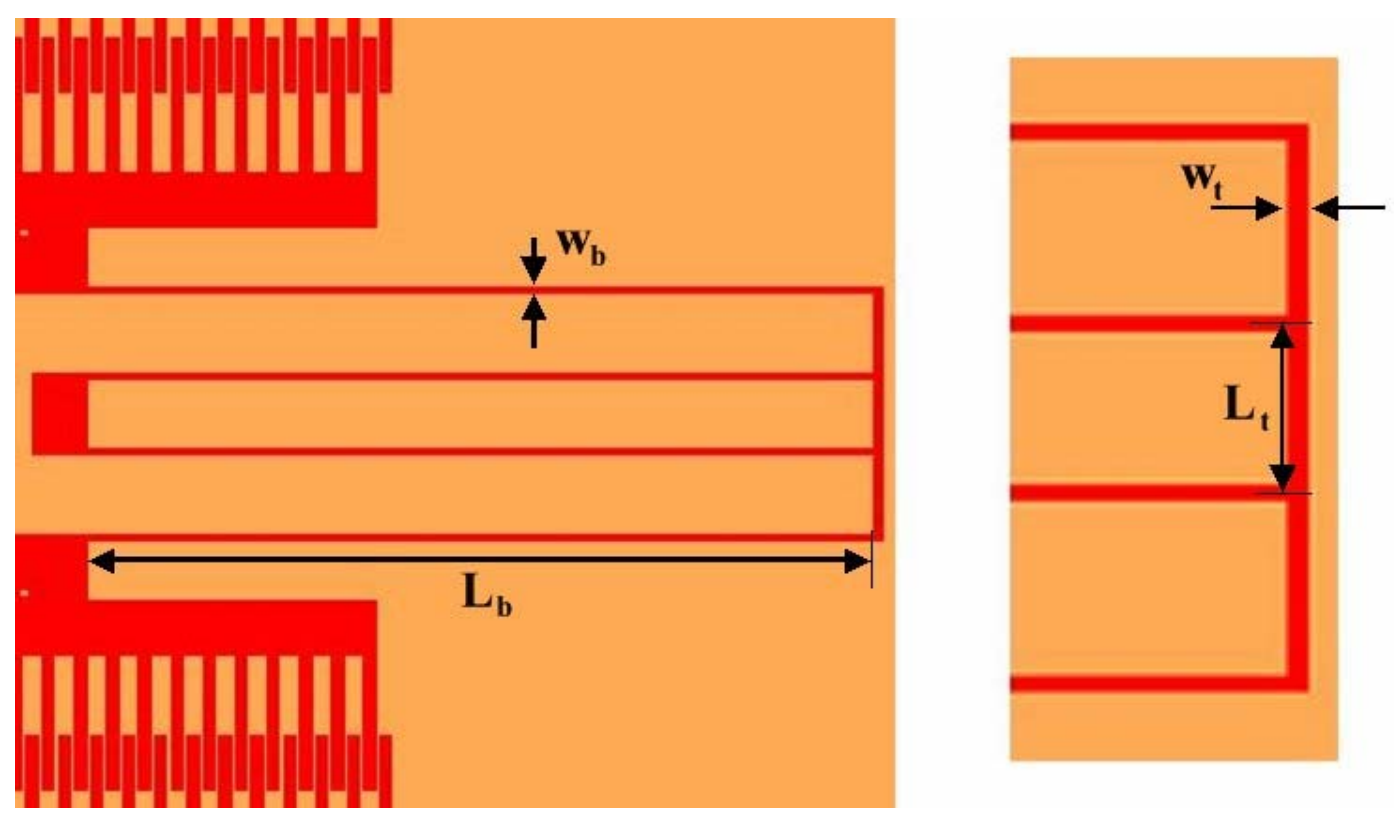

Figure 2.1: Flexure and truss dimensions.

\section{Spring Constant}

While the spring constant of the folded flexure design applied in the devices used for this research has a component in the $x, y$, and $z$-directions, the component in the $x$-direction is dominant, again because of the lateral direction of motion. The spring constant in the $x$ direction can be determined by [47].

$$
k_{x}=\frac{2 E t w_{b}^{3}}{L_{b}^{3}} \cdot \frac{L_{t}^{2}+14 \alpha L_{t} L_{b}+36 \alpha^{2} L_{b}^{2}}{4 L_{t}^{2}+41 \alpha L_{t} L_{b}+36 \alpha^{2} L_{b}^{2}},
$$

with

$$
\alpha=\left(\frac{w_{t}}{w_{b}}\right)^{3}
$$

It should be noted that Equation 2.1-3 is obtained by assuming all sections of trusses are of equal length, $L_{t}$. In the case of MEMS designed for this research, the length of the center truss varies from the length of the two outer trusses by $4 \mu \mathrm{m}$. To overcome this inequality, an average truss length value was used for $L_{t}$ in Equation 2.1-3. Spring constants calculated in this manner showed insignificant variation from the value obtained by using the non-equal truss equation presented in [48]. Since beam width and truss width are equal in this design, $\alpha$ 
has a value of 1. Using the mass calculated by Equation 2.1-2 and the spring constant from Equation 2.1-3, the resonant frequency can be found using

$$
\omega_{R}=\sqrt{\frac{k}{m}} .
$$

\section{Damping Coefficient}

The damping coefficient in the $x$-direction can be calculated by [48]

$$
\beta_{x}=\mu\left[\left(A_{s}+\frac{A_{t}}{2}+\frac{A_{b}}{2}\right) \cdot\left(\frac{1}{d}+\frac{1}{\delta}\right)+\frac{A_{c}}{g}\right]
$$

where $\mu$ is the viscosity of air, $A_{s}$ is the surface area of the stage, $A_{t}$ is the surface area of the trusses, $A_{b}$ is the surface area of the flexure beams, $d$ is the oxide thickness gap, $\delta$ is the penetration depth of the airflow above the stage, $A_{c}$ is the surface area of the comb finger sidewalls, and $g$ is the finger-to-finger gap. Because many of these values are difficult to determine with accuracy due to fabrication process tolerance, the damping coefficient can best be determined experimentally.

The state variable representation of the forced harmonic oscillator is given by

$$
\begin{gathered}
\dot{x}=x_{2}, \\
\dot{x}_{2}=\frac{1}{m}\left[-\beta x_{2}-k_{s} x_{1}+F_{e}\right], \\
\ddot{x}=\frac{1}{m}\left[-\beta \dot{x}-k_{s} x+F_{e}\right],
\end{gathered}
$$

with

$$
F_{e}=4 \varepsilon_{0} \frac{t}{g} n V_{b} V_{s}
$$

The electrostatic force, $F_{e}$, is determined using the permittivity of free space, $\varepsilon_{0}\left(8.854 \times 10^{-12}\right.$ $\mathrm{C}^{2} / \mathrm{N} \cdot \mathrm{m}^{2}$ ), the vertical thickness of the comb fingers, $t$, the finger-to-finger gap $g$, the applied DC voltage $V_{b}$, and the applied AC voltage $V_{s}$. To develop the input to output transfer function, the Laplace transform must be taken, 


$$
s^{2} X(s)=\frac{1}{m}\left[-\beta S X(s)+k_{s} X(s)+F_{e}(s)\right]
$$

resulting in

$$
\frac{X(s)}{F_{e}(s)}=\frac{1}{\left(m s^{2}+k_{s}+\beta s\right)} .
$$

The frequency-space representation of this relation is

$$
\frac{X(\omega)}{F_{e}(\omega)}=\frac{1}{\left(-m \omega^{2}+k_{s}+j \beta \omega\right)}=G(\omega)
$$

which expands to

$$
G(\omega)=\frac{-m \omega^{2}+k_{s}}{\left(-m \omega^{2}+k_{s}\right)^{2}+(\beta \omega)^{2}}-j\left[\frac{\beta \omega}{\left(-m \omega^{2}+k_{s}\right)^{2}+(\beta \omega)^{2}}\right]
$$

The gain and phase shift of the output are given by

$$
|G(\omega)|=\sqrt{\operatorname{Re}(G(\omega))^{2}+\operatorname{Im}(G(\omega))^{2}}
$$

and

$$
\angle G(\omega)=\tan ^{-1}\left(\frac{\operatorname{Im}(G(\omega))}{\operatorname{Re}(G(\omega))}\right)
$$

respectively. This information can be curve fitted to experimental gain and phase shift data using calculated mass and spring constant values and a damping value that results in the best fit.

Variations of mass and spring constant in Equation 2.1-16 will change the frequency at which resonance will occur. Variations in the damping parameter will also change the resonant frequency, as well as affect the 'steepness' of the gain curve (similar in appearance to Figure 1.9). At low frequencies, sinusoidal input drive voltage and stage displacement are in phase (from Equation 2.1-17). As resonance is approached, the two become increasingly out of phase, reaching $90^{\circ}$ at resonance. This continues until, far above resonance, sinusoidal drive voltage and displacement become $180^{\circ}$ out of phase. 


\section{Vertical Stage Motion}

Although the majority of device stage motion is in a lateral direction, there is a small amount of vertical translation as the stage moves from side to side. If vertical motion becomes to large, due to either increased drive voltage or frequency, the fingers could become disengaged and cause device failure. Even with small amplitudes of vertical motion, a change in optical output intensity due to the changing thickness of the air gap can occur (etaloning). Because of the normal angle of incidence of the probe beam, the air gap can act as both a resonance cavity and anti-reflection layer, depending on the separation distance. The extent of motion can be measured accurately during device operation using a laser vibrometer, but an estimate of vertical motion can be made using previous experience with the lateral comb resonator devices used in this research.

As the stage moves, a number of vertical motion modes can occur, three of which are pictured in Figure 2.2.

(a)

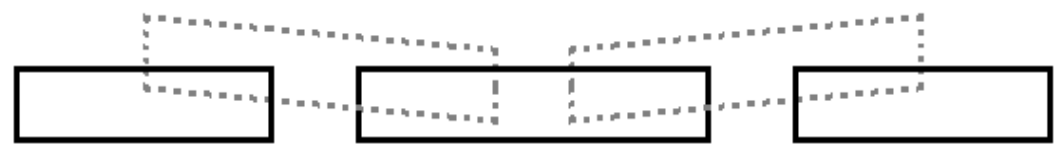

(b)

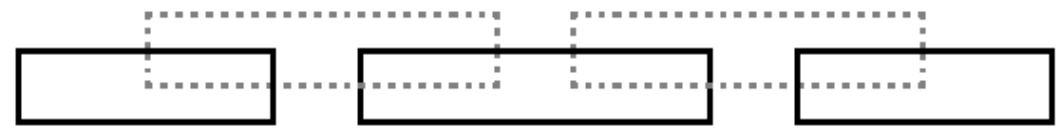

(c)
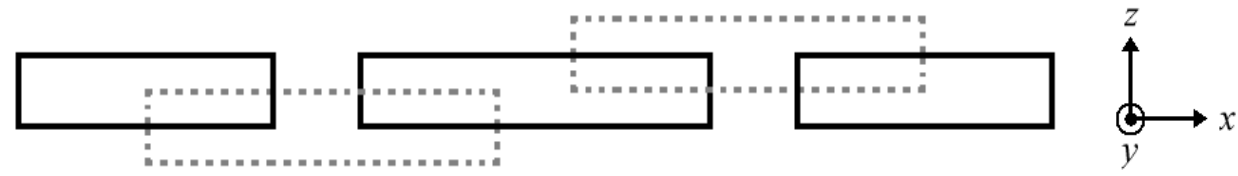

Figure 2.2: Theorized types of vertical stage motion: (a) raising motion with torsional bending, (b) parallel raising motion, and (c) parallel raising and lowering.

For this examination, simple vertical motion without torsional bending will be considered. In order for device failure from finger disengagement to occur, the stage must travel a minimum of $2 \mu \mathrm{m}$ along the $z$-axis over as much as $20 \mu \mathrm{m}$ of total displacement in the $x$-direction. The 
devices used in this research rarely fail under normal operating conditions, indicating that vertical motion must be less than $2 \mu \mathrm{m}$, as described in Section 4.4.4.

The change in through-wafer optical intensity caused by vertical stage motion can be determined using the reflectivity of a simple plane interface at normal incidence, given by [49]

$$
r_{12}=\frac{n_{1}-n_{2}}{n_{1}+n_{2}},
$$

where $n_{1}$ and $n_{2}$ are the indices of refraction of the incident and transmitted media respectively. The reflectance can then be found using

$$
R=\frac{r_{12}^{2}+r_{23}^{2}+2 r_{12} r_{23} \cos 2 \beta}{1+r_{12}^{2} r_{23}^{2}+2 r_{12} r_{23} \cos 2 \beta},
$$

where $r_{12}$ is the reflectivity of the first interface and $r_{23}$ is the reflectivity of the second interface, both given by $2.1-18$, and

$$
\beta=\frac{2 \pi n_{\text {flim }} h}{\lambda_{0}},
$$

with $\lambda_{0}$ being the wavelength of the beam, $h$ as the thickness of the layer, and $n_{\text {fil }}$ its refractive index. Transmittance, $T$, can be solved for using

$$
T=1-R .
$$

By using off-axis Fresnel zone plate lenses and non-normal incident probe beam for throughwafer probing, the effects of the air gap acting as a resonance cavity are reduced, but may cause signal interference in the form of multiple reflections present in the air gap, similar to the condition pictured in Figure 2.3. 


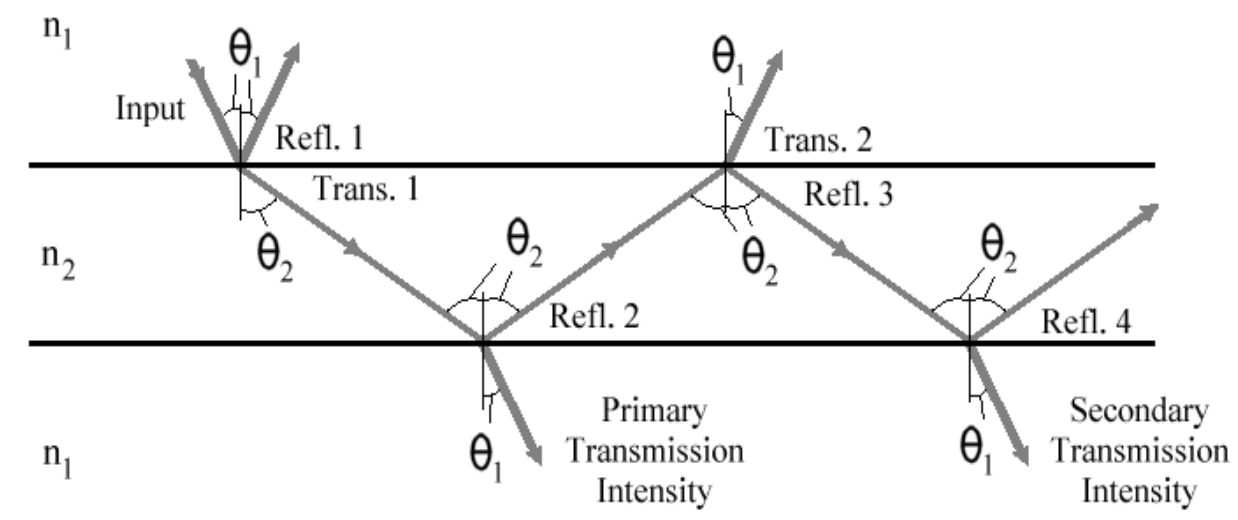

Figure 2.3: Angular incidence interaction at a planar boundary resulting in multiple reflection and transmission paths.

The reflectivity of a planar interface for a TE wave of angular incidence is given by [50]

$$
r=\frac{n_{1} \cos \theta_{1}-n_{2} \cos \theta_{2}}{n_{1} \cos \theta_{1}+n_{2} \cos \theta_{2}} .
$$

Propagation angle in the second medium, $\theta_{2}$, can be found using Snell's Law,

$$
n_{1} \sin \theta_{1}=n_{2} \sin \theta_{2} .
$$

Reflectance and transmittance can then be obtained using

$$
R=\left|r^{2}\right|
$$

and Equation 2.1-21. By tracing the propagation path of the primary ray and its reflections while adding the effects of reflection losses, the relative intensities of the secondary transmission paths and their proximity to the primary transmitted ray can be found.

\subsection{Examination of Diffraction Effects Caused by MEMS Device Stage Features}

In order to gain a better understanding of the effect that diffraction has on a position signal obtained via through-wafer probing, it is necessary to examine basic diffraction theory. There are three main factors that play a role in diffraction. The first is the complex amplitude of the input beam $U(r)$. The next is the pupil function, $p(x, y)$, or transmittance function $t(x, y)$, both of which are developed based on the features of the of the device stage. The final factor 
is the transfer function of free space, $h(x, y)$, which determines the amplitude and shape of the beam at some distance $d$ away from the device stage.

\section{Plane Wave Diffraction by a Slit}

To begin, a simple plane wave incident on a slit is examined [50]. The complex amplitude of a plane wave is given by

$$
U(r)=A \exp (-j k z)
$$

where $A$ is the wave amplitude, $k=2 \pi / \lambda$ is the wave number, and $z$ is the distance away from the source.

The geometry of the slit is defined as a pupil function. The pupil function of the slit can be expressed as

$$
p(x, y)=\left\{\begin{array}{l}
1,|x| \leq a \\
0, \text { elsewhere }
\end{array}\right.
$$

where $a$ is the half-width of the slit.

The aperture function, $f(x, y)$, is simply an expression of the input waveform shadowed by the pupil,

$$
f(x, y)=U(r) p(x, y) .
$$

By convolving this aperture function with the Fresnel approximation of the transfer function of free space,

$$
h(x, y) \approx h_{0} \exp \left[-j k \frac{x^{2}+y^{2}}{2 d}\right]
$$

where $h_{0}=(j / \lambda d) \exp (-j k d)$, the output function, $g(x, y)$, that defines an observation plane at a distance $d$ can be obtained. It is also often useful to look at the Fresnel number, $N_{F}=a^{2} / \lambda d$, which relates the half-width of the slit, $a$, to the observation plane distance. 


\section{Rectangular Aperture}

The same procedure can be followed to examine the diffraction of a plane wave passing through a rectangular aperture. The pupil function is defined as

$$
p(x, y)= \begin{cases}1, & |x| \leq a,|y| \leq b \\ 0, & \text { elsewhere }\end{cases}
$$

where $a$ and $b$ are the width and length of the slit, respectively. The rectangular aperture adds another degree of dimensionality to the pupil function, resulting in diffraction from all sides of the opening.

\section{Transparent Media}

Optical rays will also experience diffraction when passing through a transparent medium. In order to fully examine the through-wafer probe setup, the stage must be modeled as a transmission function. Using only a pupil function does not account for the actual physical thickness and index of refraction of the poly stage, which are certain to enhance diffraction effects by further retarding the phase of the beam. In order for index of refraction and layer thickness to be included, the pupil function must be replaced with a complex amplitude transmittance [50],

$$
t(x, y)=\exp (-j n k t)
$$

where $n$ is the index of refraction, $k$ is the wave number, and $t$ is the thickness of the stage. This can be a continuous function, or piecewise defined similar to the pupil function mentioned earlier.

\section{Gaussian Beam Diffraction}

In the current through-wafer probing setup, the output of a fiber is focused to a small spot and passed through the MEMS device stage. This beam is assumed to be Gaussian, having a complex amplitude that can be expressed as [1]

$$
U(r)=A_{0} \frac{W_{0}}{W(z)} \exp \left[-\frac{x^{2}+y^{2}}{W^{2}(z)}\right] \exp \left[-j k z-j k \frac{x^{2}+y^{2}}{2 R(z)}+j \zeta(z)\right],
$$


with beam parameters

$$
\begin{gathered}
W(z)=W_{0}\left[1+\left(\frac{z}{z_{0}}\right)^{2}\right]^{1 / 2}, \\
R(z)=z\left[1+\left(\frac{z_{0}}{z}\right)^{2}\right], \\
\zeta(z)=\tan ^{-1} \frac{z}{z_{0}}, \\
W_{0}=\left(\frac{\lambda z_{0}}{\pi}\right)^{1 / 2},
\end{gathered}
$$

and

$$
A_{0}=\frac{A}{j z_{0}},
$$

where $W(z)$ is the beam width a distance $z$ on the optical axis, $W_{0}$ is the beam width at the Raleigh range, $z_{0}$, and $R(z), \zeta(z)$, and $A_{0}$ are the radius of curvature, phase, and amplitude of the wavefront at distance $z$, respectively. The complex Gaussian beam amplitude (Equation 2.2-7), the transmission function of the stage (obtained from Equation 2.2-6), and the transfer function of free space are used to determine the optical field intensity at a distance, $d$, away from the device stage by first finding the aperture function and convolving it with the transfer function of free space.

\subsection{Fresnel Lens Zone Pattern Determination}

On-Axis Lenses

On-axis focusing Fresnel lenses consist of areas of varying substrate etch depth radially symmetrical around a central point, as illustrated in Figure 2.4. 


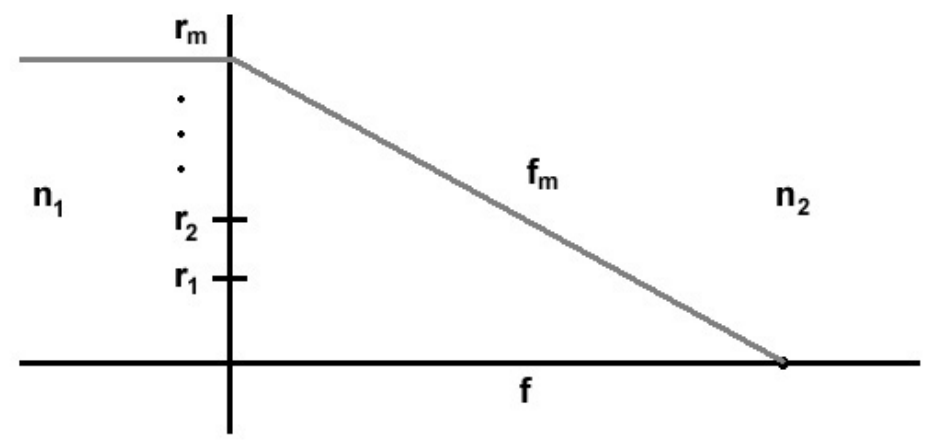

Figure 2.4: On-axis Fresnel lens system.

In this configuration, collimated rays entering the lens experience a phase shift due to the different substrate thickness at each radius and are focused at a distance $f$. Determination of the radii defining the edges of the zones involves examining the optical path difference (OPD) between the ray passing through the center of the lens to the focal point (along the optical axis) and the ray passing through each $r_{m}$ to the focal point. This is expressed as

$$
O P D=f_{m}-f,
$$

where

$$
f_{m}=\sqrt{f^{2}-r_{m}^{2}},
$$

leading to

$$
O P D=\sqrt{f^{2}-r_{m}^{2}}-f .
$$

The optical path difference is chosen to be a fraction of the wavelength of light used in the system, depending on the number of phase levels desired for the lens. Figure 2.5 shows the relationship between OPD and the phase difference at each radii of the lens for a four level zone plate lens. 


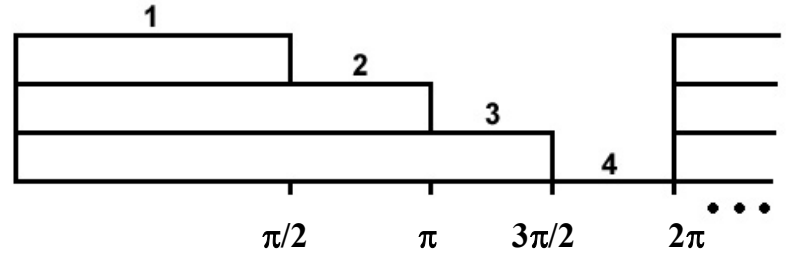

(a)

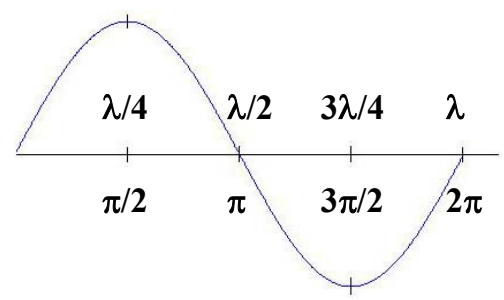

(b)

Figure 2.5: (a) Zone pattern cross-section illustrating phase levels. (b) Wavelength to phase shift comparison for OPD determination.

In this case, there are four levels of the zone pattern in the substrate, so the wavelength has been divided into multiple orders of fourths for OPD determination. For binary, or two-level, zone patterns, the wavelength is divided into multiple orders of half wavelengths. Increasing the number of phase levels of the lens increases its focusing efficiency, so the number of levels should be chosen according to the requirements of the system [51]. Once the desired focal length, operating wavelength, and number of phase levels of the lens have been determined, the radii can be determined by substituting the multiple order of fractional wavelengths for OPD into Equation 2.3-3 and solving for $r_{m}$ [52]. Table 2.1 lists the equations developed for the determination of the radii for the lens cross-section shown in Figure 2.5.

Table 2.1: Zone radius equations for various phase shifts.

\begin{tabular}{|c||c|c|}
\hline Phase Shift & OPD & $\boldsymbol{r}_{m}$ \\
\hline$\pi / 2$ & $m \lambda / 4$ & $r_{m}=\sqrt{f \frac{m \lambda}{2}+\left(\frac{m \lambda}{4}\right)^{2}}$ \\
\hline$\pi$ & $m \lambda / 2$ & $r_{m}=\sqrt{f m \lambda+\left(\frac{m \lambda}{2}\right)^{2}}$ \\
\hline $3 \pi / 2$ & $3 m \lambda / 4$ & $r_{m}=\sqrt{f \frac{3 m \lambda}{2}+\left(\frac{3 m \lambda}{4}\right)^{2}}$ \\
\hline $2 \pi$ & $m \lambda$ & $r_{m}=\sqrt{2 f m \lambda+(m \lambda)^{2}}$ \\
\hline
\end{tabular}

Maximum etch depth corresponds to a phase retardation of $2 \pi$ for a four level lens, leading to the relation [53] 


$$
d_{\max }=\frac{\lambda}{(n-1)}
$$

This can be adjusted for lenses having less than four levels by using the proper fraction of Equation 2.3-4.

Off-Axis Lenses

For the case of off-axis Fresnel lenses, the model of a thin lens with light entering only half of the lens was used (adapted from [54]), illustrated in Figure 2.6. It should be noted that this type of lens is not symmetrical around a central point, as was the case with on-axis lenses.

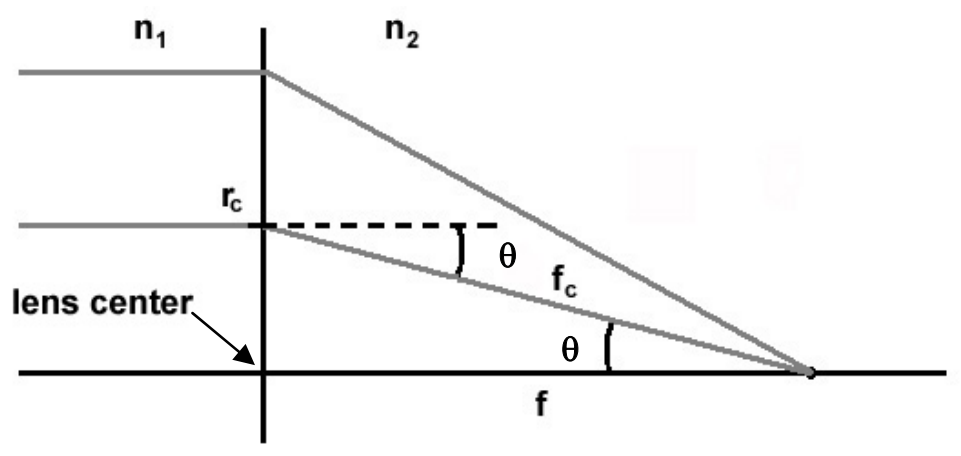

Figure 2.6: Off-axis lens arrangement.

An off-axis lens is essentially an area of an on-axis lens centered on a central radius determined by the amount of off-axis focal point deflection. This central radius can be found by

$$
r_{c}=f \tan \theta
$$

where $\theta$ is the desired angle of deflection (in this case $\geq 41.8^{\circ}$, the total internal reflection angle for the quartz substrate the lenses will be fabricated in). To define the off-axis radii, an on-axis lens is created first. Then, using the total internal reflection angle and a desired focal length, the radius that will be defined as the center of the lens can be found. Because of the difficulty in achieving this small linewidth consistently in the photolithography process, the input angle will have to be relaxed (i.e. not collimated) to achieve reasonable photoresist linewidths (Figure 2.7). 


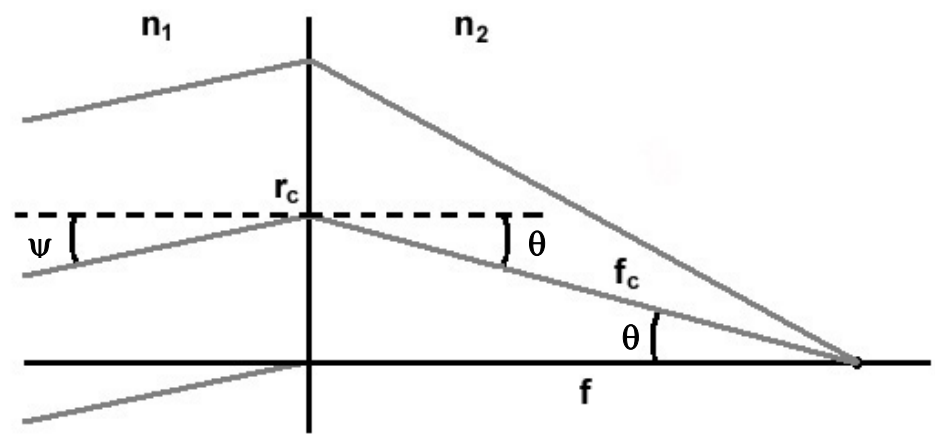

Figure 2.7: Relaxed input illustration for off-axis lenses.

Since the input beam is no longer incoming at zero degrees with respect to the optical axis, Snell's law must be used to account for the differences in indices of refraction between glass and air. The relation presented by Equation 2.1-23 can be used to determine the input angle, $\psi$, of light that, when coupled with the deflection angle of the lens, will result in a total internal reflection condition.

Off-axis focusing will result in comatic and astigmatic aberration of the focused beam due to the non-paraxial nature of the incoming optical wavefronts, with coma being the major aberration due to the large number of zones used to create the lens. Comatic aberration is illustrated in Figure 2.8.

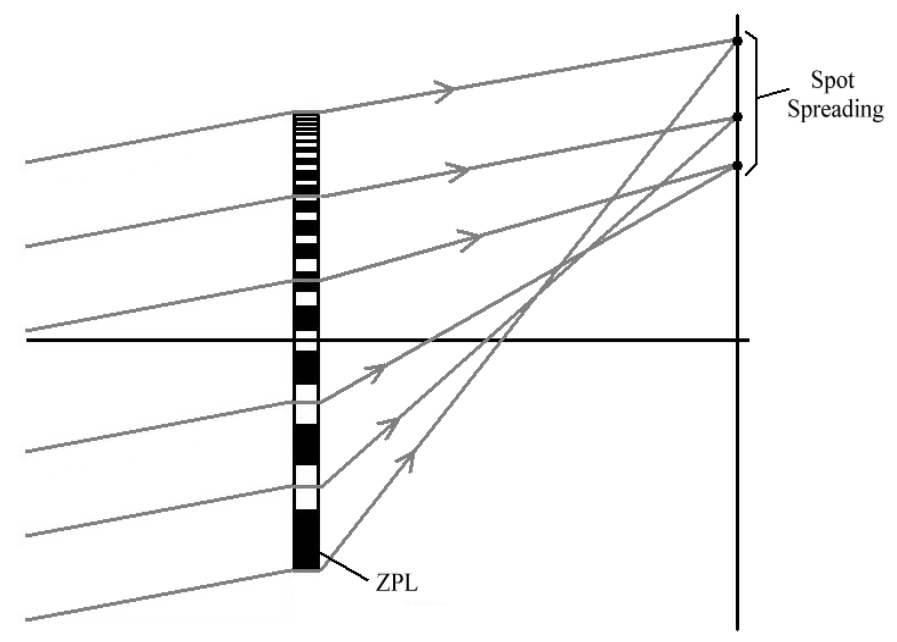

Figure 2.8: Spot spreading due to comatic aberration.

The amount of comatic aberration can be determined by examining the power series expansion of Equation 2.3-1. The third term in the expansion for off-axis zone plate lenses 
describes the coma. Using this term, the off-axis focus angle $\alpha$ that will result in coma can be solved for, giving [55]

$$
\alpha=\sqrt{\frac{n \lambda}{f}}\left(\frac{1}{2 n}\right),
$$

where $n$ is the number of zones in the lens, $\lambda$ is the wavelength of the beam, and $f$ is the focal length of the lens. This aberration will be acceptable in early IOM process development, but will have to be taken in to consideration in subsequent efforts by using a more complicated diffraction pattern.

\subsection{Fiber-to-Fiber and Laser Diode-to-Fiber Coupling Efficiency Examination}

Optical monitoring of MEMS involves the employment of common optical system elements, either bulk or integrated, to probe devices and receive the positional information. The current MEMS optical monitoring system employed by our research group uses fiber optics to input and collect the probe beam. This will be improved in the future by using integrated Fresnel zone plate lenses, creating an optical monitoring system that is decoupled from the MEMS and can be employed over the lifetime of the device. Because of diffraction effects caused by the surface features of the moving stage, the position of the receiving element in relation to the optical input element is important. If the collector element is far away, the optical beam may be deflected completely out of the acceptance range of the receiving element. Even if these diffraction effects are ignored, the amount of light coupled decreases as the distance between the optical output and receiving element is increased. This section presents an examination of how coupling efficiency is affected by increasing the distance between output and receiver for the simple cases of fiber to fiber and laser diode (LD) to fiber coupling. 


\subsubsection{Optical Output Element}

For this study, the output to receiver separation will be represented by displacement on the z-axis, with $x$ and $y$-axis misalignments assumed to be negligible. The fiber optical output is assumed to be circular Gaussian, with complex amplitude that can be expressed using Equation 2.2-7 - 2.2-12. Since $W_{0}$ can be approximated $(\sim 4.1 \mu \mathrm{m}$ for the current optical monitoring setup), the $\operatorname{sqrt}(2)^{*} W_{0}$ distance $z_{0}$ can be solved for using equation 2.1.5, resulting in

$$
z_{0}=\frac{\pi W_{0}^{2}}{\lambda}
$$

The intensity of this beam at a distance of $100 \mu \mathrm{m}$ away from the output is illustrated in Figure 2.9.

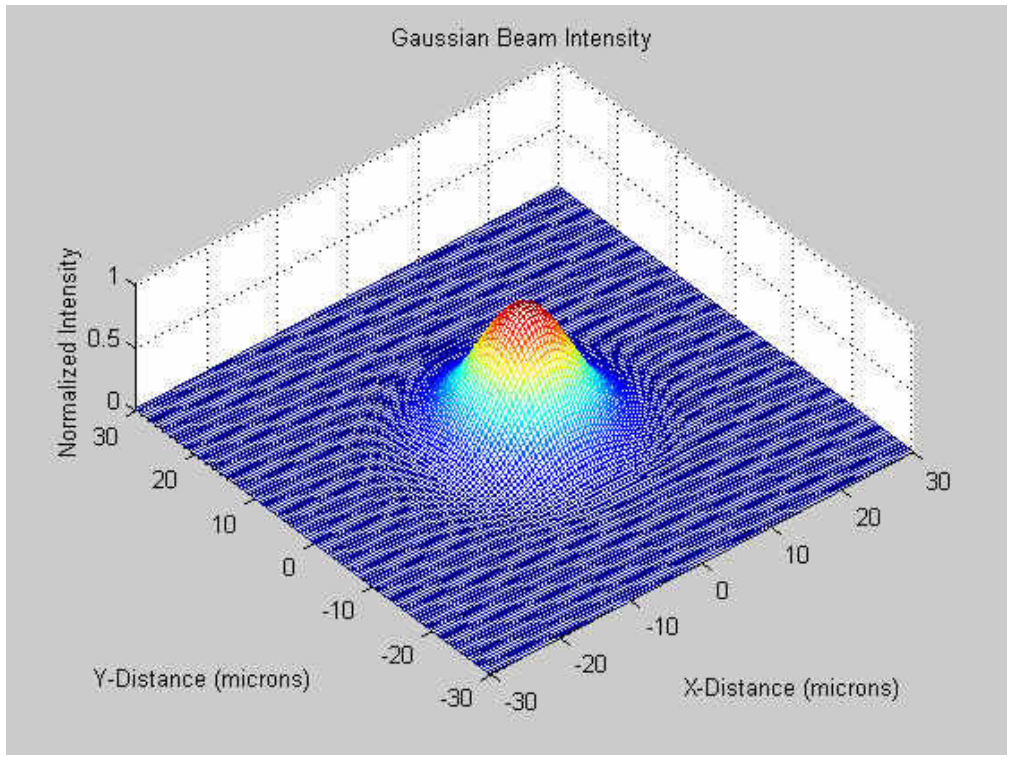

Figure 2.9: Circular Gaussian Beam Intensity at $100 \mu \mathrm{m}$.

The output of the laser diode is assumed to be an elliptical Gaussian with complex amplitude represented by

$$
U_{L D}(x, y)=A_{0} \frac{W_{x, 0} / W_{y, 0}}{W_{x}(z) / W_{y}(z)} \exp \left[\frac{-x^{2}}{W_{x}^{2}(z)}+\frac{-y^{2}}{W_{y}^{2}(z)}\right] \exp \left[-j k z-j k\left(\frac{x^{2}}{2 R_{x}(z)}+\frac{y^{2}}{2 R_{y}(z)}\right)+j \zeta_{x}(z)+j \zeta_{y}(z)\right],
$$


with beam parameters

$$
\begin{gathered}
W_{x}(z)=W_{x, 0}\left[1+\left(\frac{z}{z_{x, 0}}\right)^{2}\right]^{1 / 2}, W_{y}(z)=W_{y, 0}\left[1+\left(\frac{z}{z_{y, 0}}\right)^{2}\right]^{1 / 2}, \\
R_{x}(z)=z\left[1+\left(\frac{z_{x, 0}}{z}\right)^{2}\right], R_{y}(z)=z\left[1+\left(\frac{z_{y, 0}}{z}\right)^{2}\right], \\
\zeta_{x}(z)=\tan ^{-1} \frac{z}{z_{x, 0}}, \zeta_{y}(z)=\tan ^{-1} \frac{z}{z_{y, 0}},
\end{gathered}
$$

and

$$
W_{x, 0}=\left(\frac{\lambda z_{x, 0}}{\pi}\right)^{1 / 2}, W_{y, 0}=\left(\frac{\lambda z_{y, 0}}{\pi}\right)^{1 / 2}
$$

calculated similarly to their circular Gaussian counterparts. The output of a laser diode with a $2 \times 10 \mu \mathrm{m}$ output area is illustrated in Figure 2.10 at a distance of $50 \mu \mathrm{m}$ away.

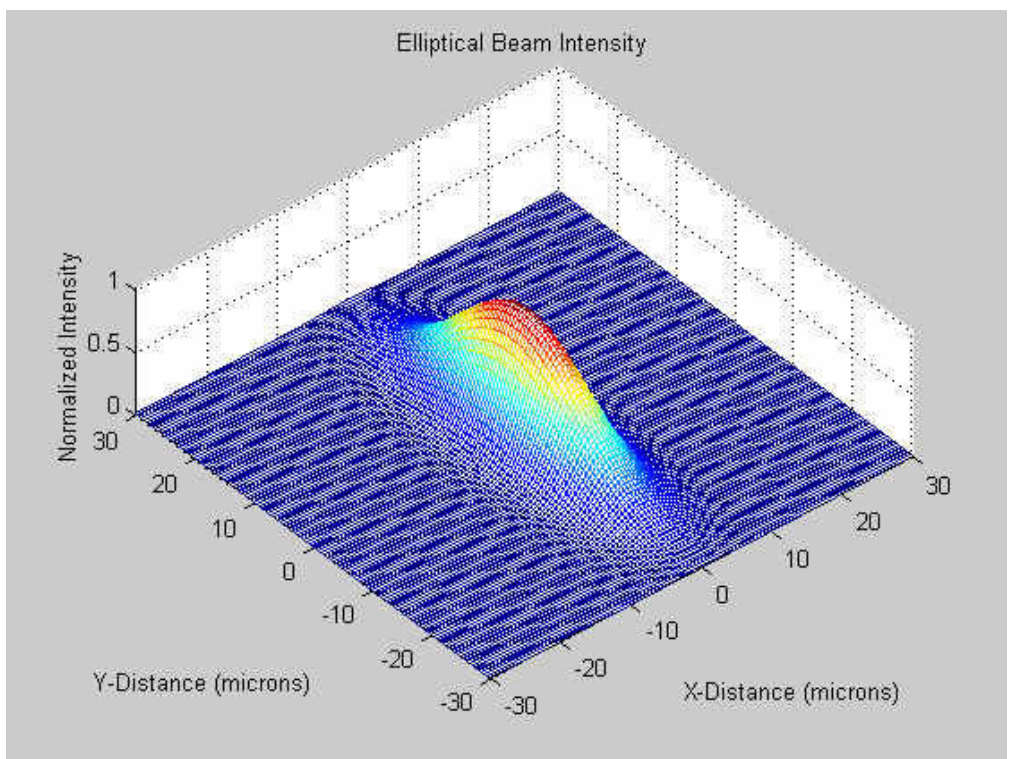

Figure 2.10: Elliptical Gaussian Beam Intensity at $50 \mu \mathrm{m}$. 


\subsubsection{Receiving Fiber}

In order to account for phase mismatch between the optical output element and the receiving fiber, the fiber optical mode field of the receiving fiber must be found. This is given by [56]

$$
U_{r F}(x, y)=A_{0} f(x, y) \exp \left(-j \beta z_{r F}\right)
$$

where $f(x, y)$ is the mode field, $\beta$ is the propagation constant in the fiber core, and $z_{r F}$ is the distance traveled in the fiber. The propagation constant in a single mode fiber is approximated by [50]

$$
\beta=\sqrt{n_{1}^{2} k^{2}-\frac{\pi^{2}}{a^{2}}},
$$

where $n_{1}$ is the index of refraction of the core, $k$ is the wave number $(2 \pi / \lambda)$, and $a$ is the radius of the core. For a single mode fiber, $f(x, y)$ is expressed as [2]

$$
f(x, y)=\left\{\begin{array}{l}
J_{0}\left(k_{T} r\right) ; r \leq a \\
R \cdot H_{0}(j \gamma r) ; r \geq a
\end{array},\right.
$$

where

$$
\begin{gathered}
k_{T}=\sqrt{n_{1}^{2} k^{2}-\beta^{2}}, \\
\gamma=\sqrt{\beta^{2}-n_{2}^{2} k^{2}}, \\
R=\frac{J_{0}\left(k_{T} a\right)}{H_{0}(j \gamma a)},
\end{gathered}
$$

and

$$
r=\sqrt{x^{2}+y^{2}}
$$

The index of refraction of the fiber cladding is defined as $n_{2}$. The ratio, $R$, allows continuity of the mode field across the core-cladding boundary. As seen in Equations 2.4-9 - 2.4-12, the expression for the mode field is highly dependent on $\beta$, which can be approximated by 
equation 2.4-8. However, this approximation causes a discontinuity at $r=a$. The exact value of $\beta$ can be determined by adjusting its value to eliminate the discontinuity (Note: an error in the fourth decimal place of $\beta$ can cause discontinuity).

To eliminate this problem, the mode field can be approximated as a Gaussian intensity distribution by [56]

$$
f(x, y)=A \exp (-r / a)^{2} .
$$

A comparison of the Bessel function determined mode field and the Gaussian distribution approximation is shown in Figure 2.11 (Appendix A, 'modeprop.m').

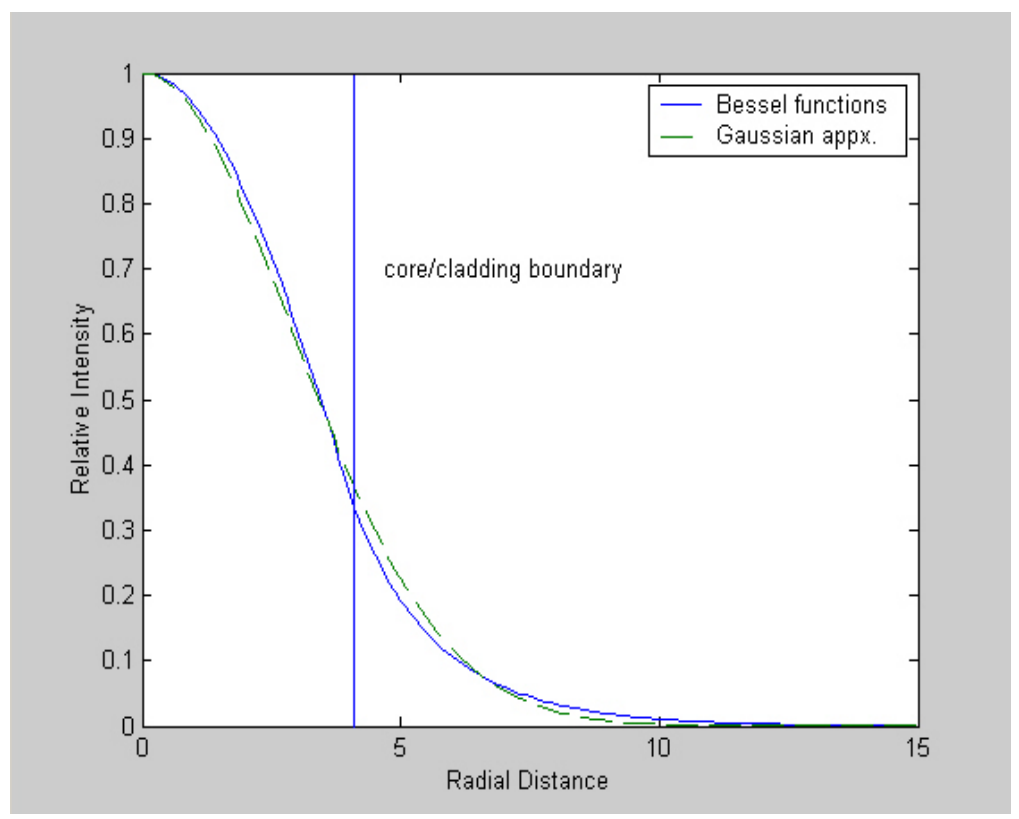

Figure 2.11: Mode Field Comparison.

Using the Gaussian expression, the fiber mode field can be expressed as

$$
U_{r F}(x, y)=A_{0} \exp \left[-\left(\frac{x^{2}+y^{2}}{a}\right)\right] \exp \left(-j \beta z_{r F}\right) .
$$




\subsubsection{Coupling Efficiency}

Determination of the coupling efficiency between the optical output source and the receiving fiber involves computing the overlap integral of the output amplitude distribution and the fiber mode field distribution. This expression is given as [57,58]

$$
\eta=\frac{\left|\iint U_{L D, o F}(x, y) U_{r F}^{*}(x, y) d x d y\right|^{2}}{\iint U_{L D, o F}(x, y) U_{L D, o F}^{*}(x, y) d x d y \iint U_{r F}(x, y) U_{r F}^{*}(x, y) d x d y} .
$$

Mathcad was used to calculate the integrals from -100 to $100 \mu \mathrm{m}$, giving

$$
\begin{gathered}
\iint U_{o F}(x, y) U_{r F}^{*}(x, y) d x d y=-e r f\left[\frac{1}{10000} \sqrt{-a_{1}+j b_{1}}\right]^{2} \frac{\pi}{a_{1}-j b_{1}} B_{1}, \\
\iint U_{o F}(x, y) U_{o F}^{*}(x, y) d x d y=\frac{1}{2} \operatorname{erf}\left[\frac{1}{10000} \frac{\sqrt{2}}{W(z)}\right]^{2} \pi W_{0}^{2},
\end{gathered}
$$

and

$$
\iint U_{r F}(x, y) U_{r F}^{*}(x, y) d x d y=\frac{1}{2} \operatorname{erf}\left[\frac{1}{10000} \frac{\sqrt{2}}{W_{0}}\right]^{2} \pi W_{0}^{2}
$$

for fiber to fiber coupling with

$$
\begin{gathered}
B_{1}=\frac{W_{0}}{W(z)} \exp \left[-j k z+j \beta z_{1}+j \zeta(z)\right], \\
a_{1}=\frac{-W_{0}^{2}-W(z)^{2}}{W_{0}^{2} W(z)^{2}}
\end{gathered}
$$

and

$$
b_{1}=\frac{k}{2 R(z)} \text {. }
$$


For laser diode to fiber coupling, integration over the same area gives

$$
\begin{gathered}
\iint U_{L D}(x, y) U_{r F}^{*}(x, y) d x d y=\operatorname{erf}\left[\frac{1}{10000} \sqrt{-a_{2}+j b_{2}}\right] \operatorname{erf}\left[\frac{1}{10000} \sqrt{-c+j d}\right] \\
\frac{\pi}{\sqrt{-a_{2}+j b_{2}} \sqrt{-c+j d}} B_{2}
\end{gathered}
$$

and

$$
\begin{aligned}
& \iint U_{L D}(x, y) U_{L D}^{*}(x, y) d x d y= \frac{1}{2} \frac{W_{x, 0} / W_{y, 0}}{W_{x}(z) / W_{y}(z)} \operatorname{erf}\left[\frac{1}{10000} \frac{\sqrt{2}}{W_{x}(z)}\right] \\
& \cdot \operatorname{erf}\left[\frac{1}{10000} \frac{\sqrt{2}}{W_{y}(z)}\right] \pi W_{x}(z) W_{y}(z)
\end{aligned}
$$

with

$$
\begin{gathered}
B_{1}=\frac{W_{x, 0} / W_{y, 0}}{W_{x}(z) / W_{y}(z)} \exp \left[-j k z+j \beta z_{1}+j \zeta_{x}(z)+j \zeta_{y}(z)\right], \\
a_{2}=\frac{-\rho^{2}-W_{x}(z)^{2}}{\rho^{2} W_{x}(z)^{2}}, \\
b_{2}=\frac{k}{2 R_{x}(z)} \\
c=\frac{-\rho^{2}-W_{y}(z)^{2}}{\rho^{2} W_{y}(z)^{2}}
\end{gathered}
$$

and

$$
d=\frac{k}{2 R_{y}(z)},
$$

where $\rho$ is the radius of the receiving fiber. The second integral term in the denominator gives the same result as Equation 2.4-19. 


\subsubsection{Complex Error Function}

Integration in the numerator of Equation 2.4-16 results in an error function containing complex arguments. Because Matlab cannot handle complex numbers as input arguments to an error function, equations from [5] were used to determine the values for the error function terms in Equations 2.4-17 and 2.4-23. They were

$$
\operatorname{erf}(X+j Y)=1-\left\{\exp \left(Y^{2}-X^{2}\right)[\cos (2 X Y)-j \sin (2 X Y)] w(-Y+j X)\right\} \quad 2.4-30
$$

and

$$
w(Z)=\sum_{n=0}^{\infty} \frac{(j Z)^{n}}{\Gamma\left(\frac{n}{2}+1\right)}, Z=X+j Y
$$

To calculate $w(Z)$, the summation in Equation 2.4-31 was carried out over 100 terms and a result that matched tabulated values was obtained.

\subsubsection{Results}

A Matlab program was written to determine the complex error functions plot fiber to fiber and laser diode to fiber coupling efficiency as a function of z-axis separation (Appendix A, 'couple.m'). The single mode fiber specifications (used for both the optical input fiber and the receiver) at $1.3 \mu \mathrm{m}$ wavelength were given as: $n_{1}=1.4677, N A=0.14, n_{2}=1.4610$, and a core diameter of $8.2 \mu \mathrm{m}$ [59]. The laser diode was assumed to have an output area of $2 \times 10$ $\mu \mathrm{m}$ with an angular divergence in the $x$-direction (width) being $\lambda_{0} /$ width (rad), and a divergence of $\lambda_{0}$ /length in the $y$-direction. Figure 2.12 shows the results of calculating Equation 2.4-16 for fiber-to-fiber separation of 0-500 $\mu \mathrm{m}$. 


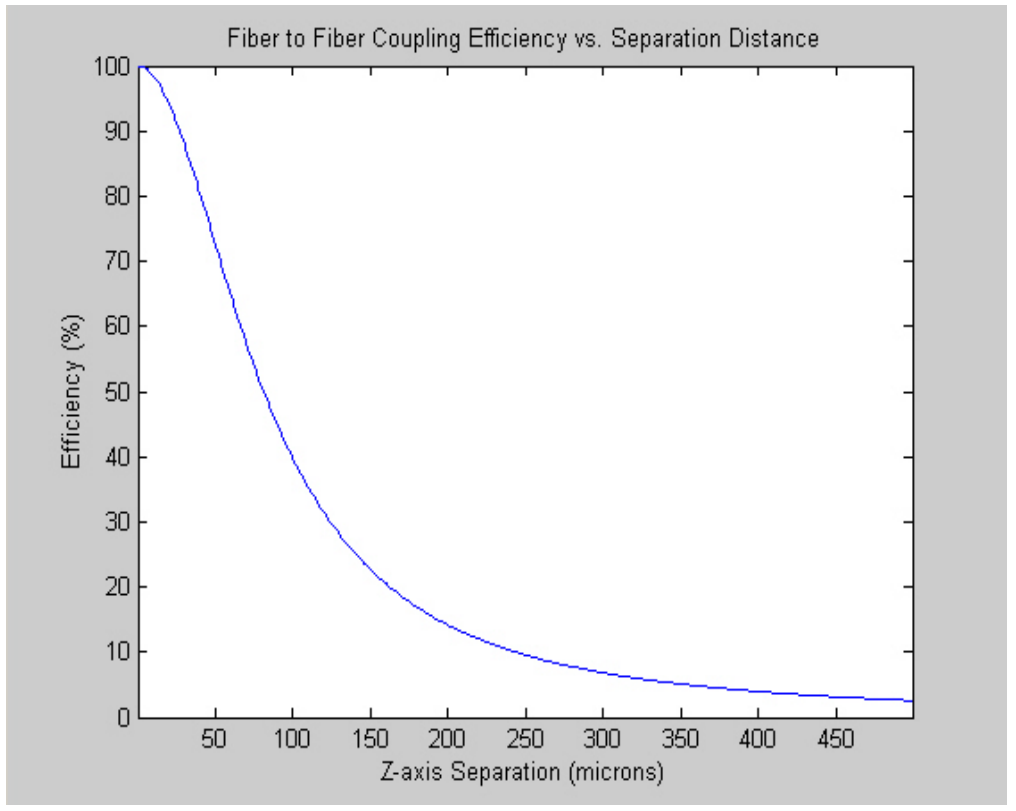

Figure 2.12: Fiber-to-fiber coupling efficiency as a function of z-axis separation.

It can be seen from Figure 2.12 that, because the optical output and receiving fibers are identical, the coupling efficiency is $100 \%$ when they are in butted contact. This falls off sharply as separation is increased. Figure 2.13 plots the coupling of a laser diode to a fiber over the same separation distance $(0-500 \mu \mathrm{m})$.

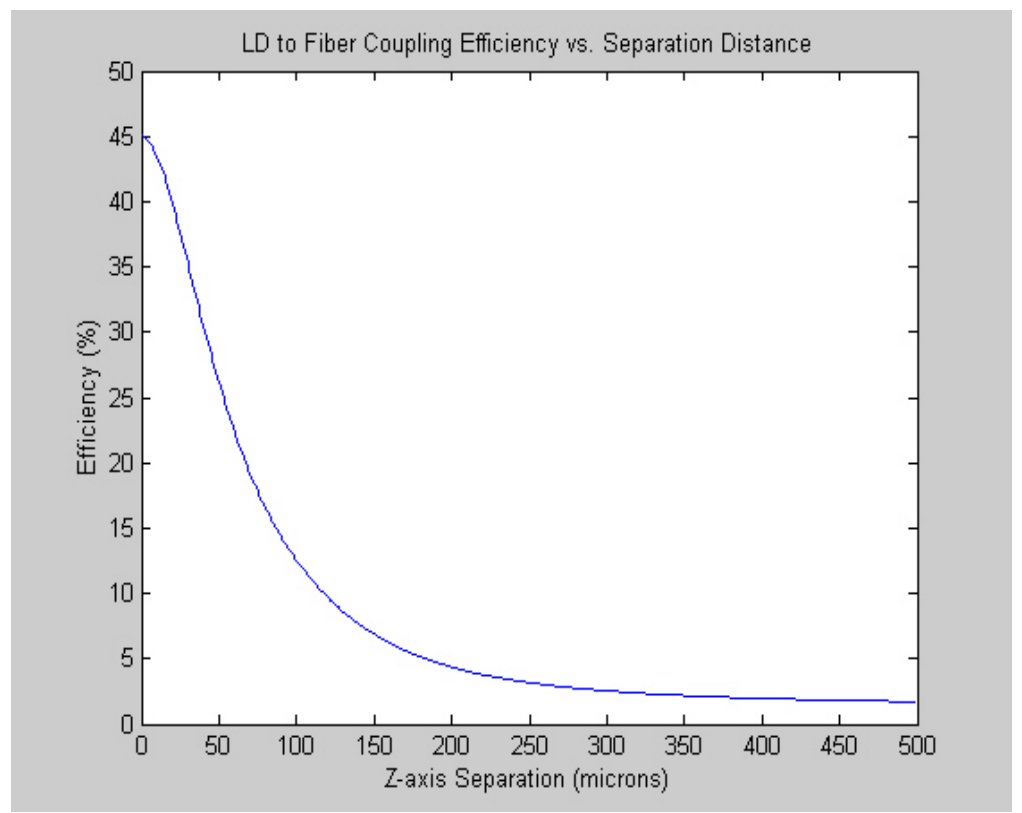

Figure 2.13: Laser diode-to-fiber coupling efficiency as a function of z-axis separation. 
Because the output intensity of the laser diode is elliptical in nature, coupling efficiency has a relatively low value, even when in contact with the receiving fiber. The size and angular divergence of the output also have an effect on the starting value. Figure 2.14 shows a comparison between fiber-to-fiber and LD-to-fiber coupling.

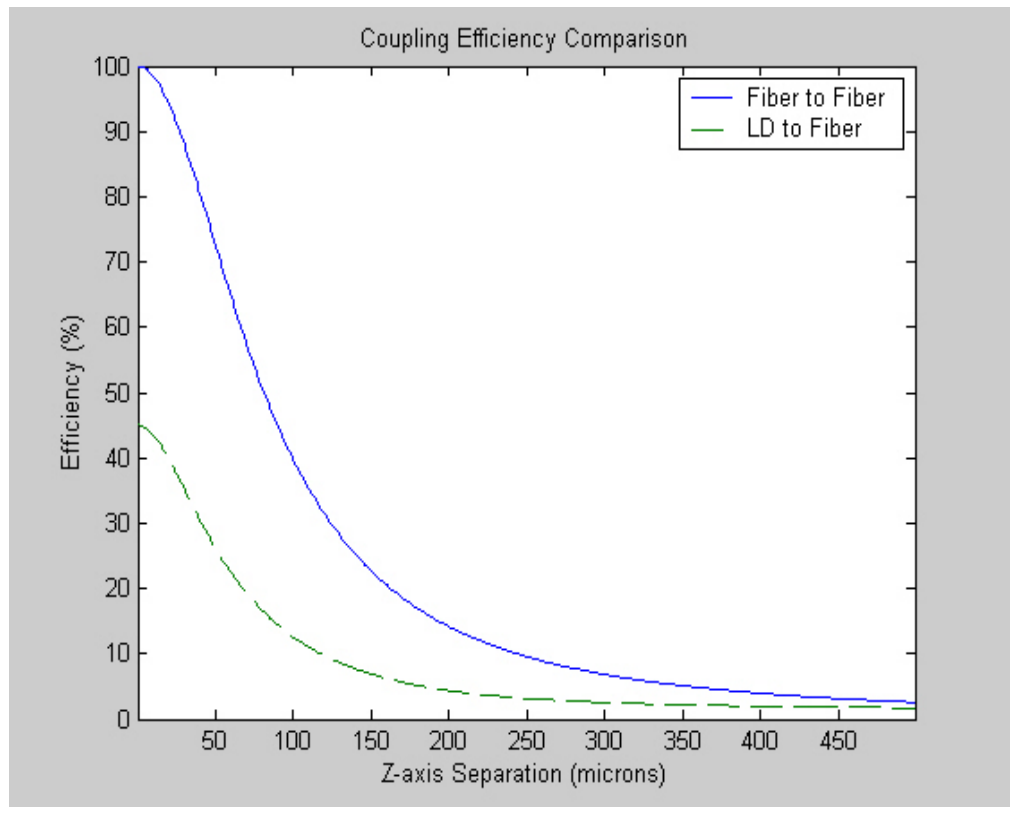

Figure 2.14: Comparison of fiber-to-fiber and LD-to-fiber coupling efficiency.

\section{Conclusion}

Coupling efficiency has been examined for two cases of basic optical element input/output configurations, fiber-to-fiber and LD-to-fiber coupling. In both cases, coupling efficiency drops greatly as a function of $z$-axis separation, mainly a result of the angular divergence of the output element. Decreasing the angular divergence with a collimating lens would reduce the rate at which coupling efficiency decreases. It has also been shown that coupling between two elements with similar input and output characteristics is more efficient than coupling between dissimilar elements. Coupling a laser diode to a fiber requires an intermediate element (such as a ball or GRIN lens) to shape the output optical waveform into one that more closely matches the receiving fiber in both amplitude distribution and phase. 


\section{Chapter 3 MEMS Lateral Comb Resonator and Optical Probe System Design}

Chapter 3 presents the parameters of the most current MUMPS MEMS lateral comb resonator design, created specifically for through-wafer optical monitoring. Basic device geometries are presented along with calculated mass and spring constant parameters. This chapter also describes mechanical reinforcement improvements made to the through-wafer optical monitoring setup to reduce external vibrational interference and to aid in the acquisition of optimum probe output signals.

\subsection{MEMS Design}

Previous through-wafer monitoring research was performed using devices designed for in-plane probing of microstructure motion. New lateral comb resonator devices were designed specifically for optimizing the dynamic range of the optical monitoring signal. The split-comb geometry of the previous design, created to allow for post-processed polymer waveguides to be added, was replace by only one set of interleaved comb fingers on each side of the device. This design lowered the surface area and, consequently, the mass, of the translation stage, allowing for higher resonant frequencies and increased lateral displacement. Figure 3.1 shows the basic geometry of the devices used in this research. 


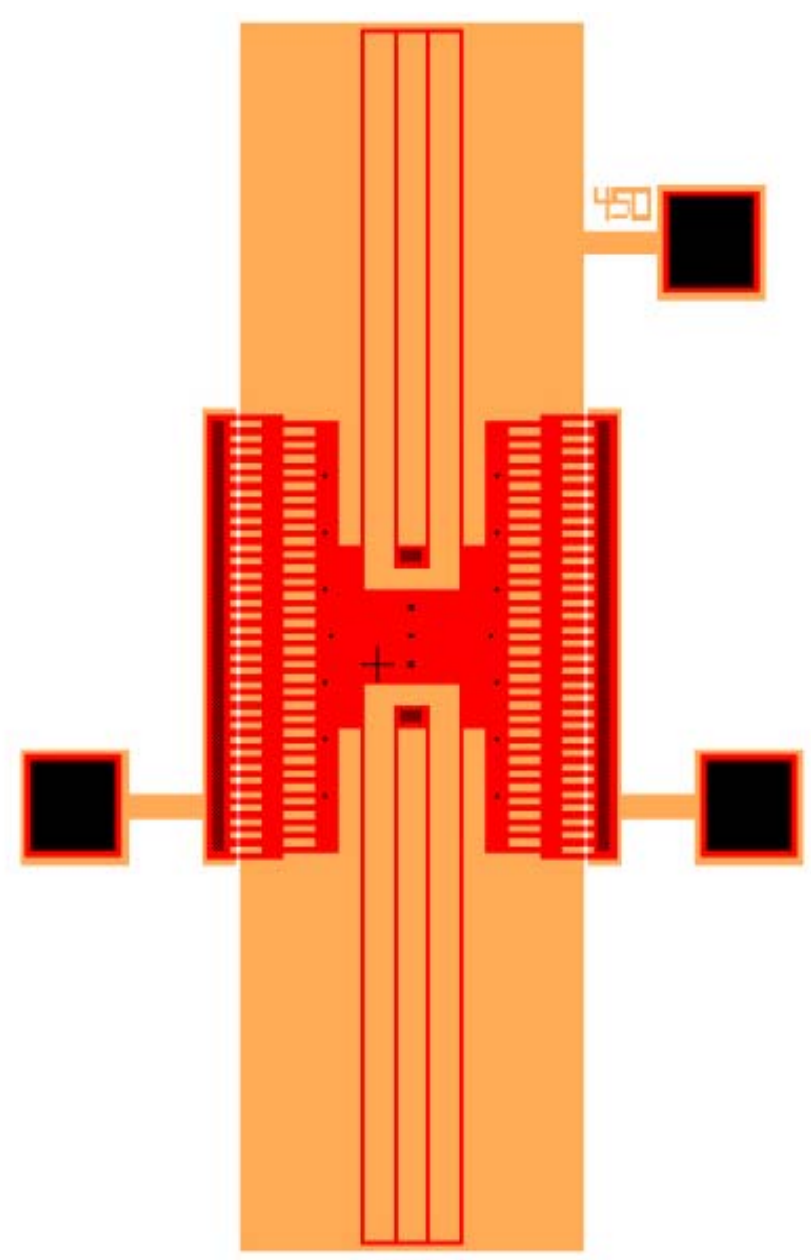

Figure 3.1: Basic comb resonator geometry.

This design has 32 sets of interleaved comb fingers on each side, and was designed with folded flexure lengths of $350,400,450$, and $500 \mu \mathrm{m}$. Two different translation stage structures were devised for obtaining the through-wafer optical signal. A 'POLY 1 openings with adjacent POLY 2 layers' arrangement was created to give high dynamic range of the signal. Some devices included metal deposited on top of the POLY 2 strips to further attenuate the probe beam. Grating structures were also employed as a means of obtaining greater positional resolution from the optical output waveform. The grating fabricated on the device translation stage consisted of 4 sections of $2 \mu \mathrm{m}$ holes and $2 \mu \mathrm{m}$ POLY 1 separations. Large grating areas were avoided to preserve the structural integrity of the stage. Both of these stage arrangements are presented in Figure 3.2. 


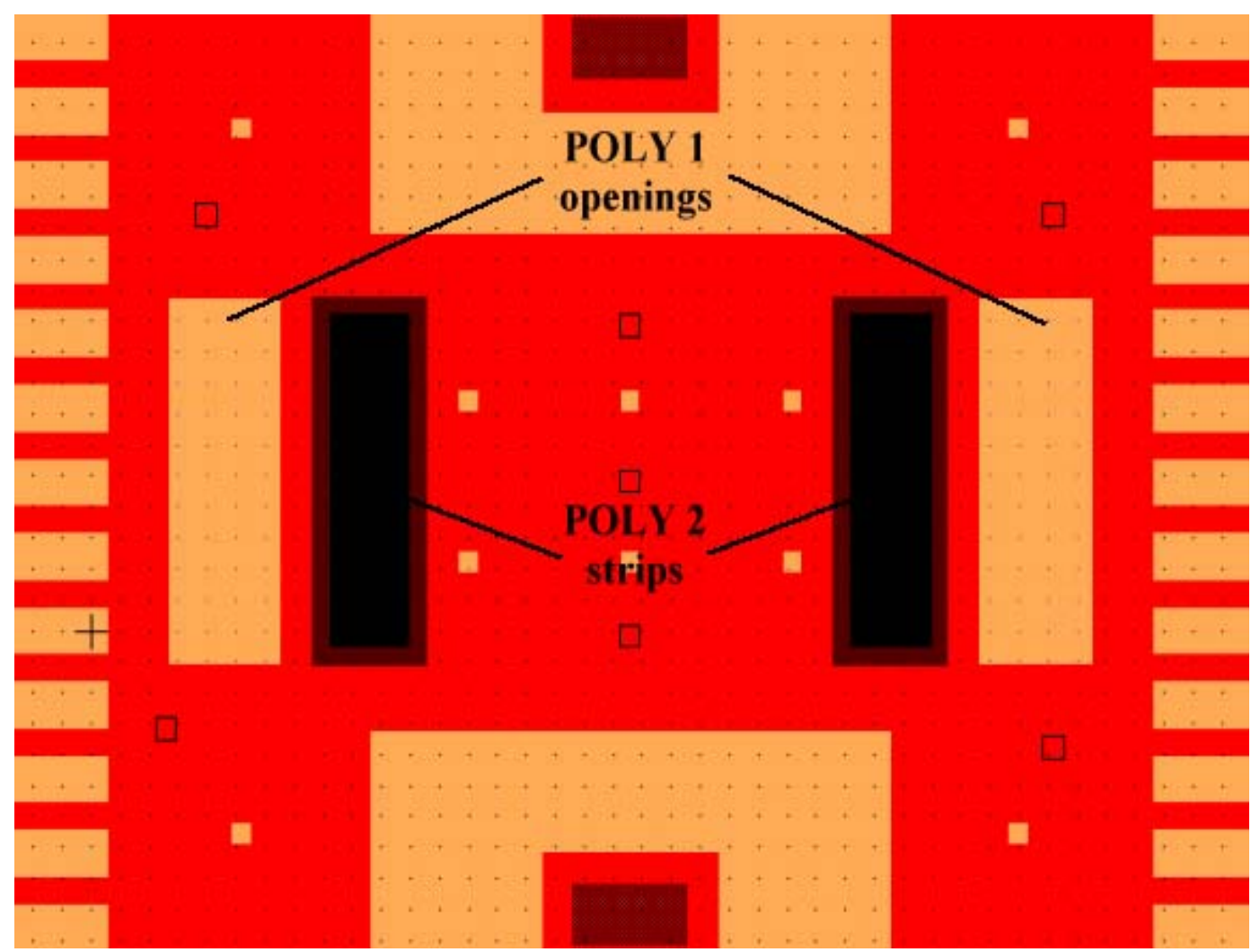

(a)

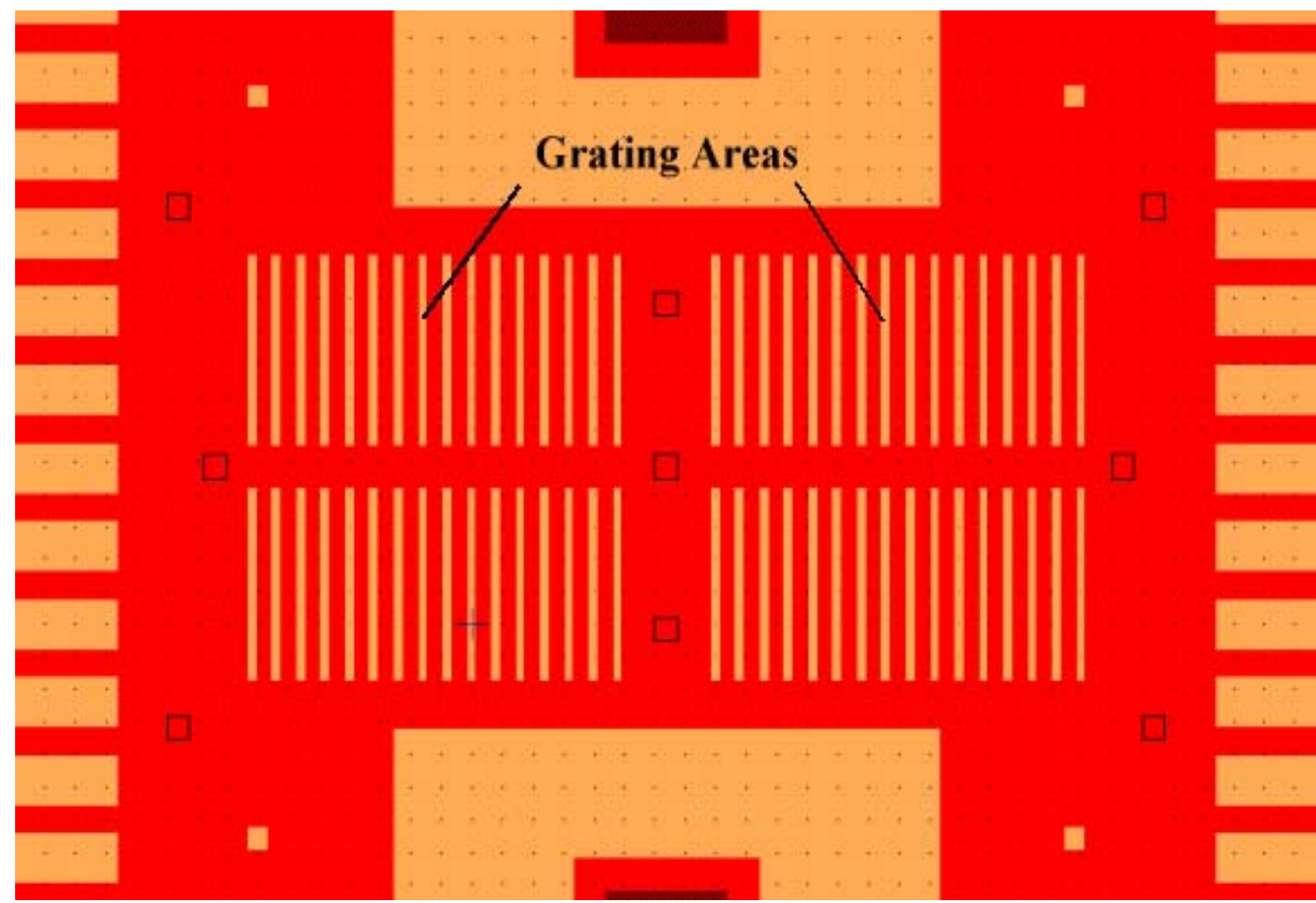

(b)

Figure 3.2: Single opening (a) and grating (b) through-wafer optical monitoring structures. 
The other types of resonator structures included in the new die layout were designed as MEMS generator devices. These devices were intended to provide an electrical signal or allow for a disturbance to be added at a set of secondary combs. Grating and single opening optical probing structures were included for through-wafer monitoring proposed. The geometry of this type of structure is shown in Figure 3.3.

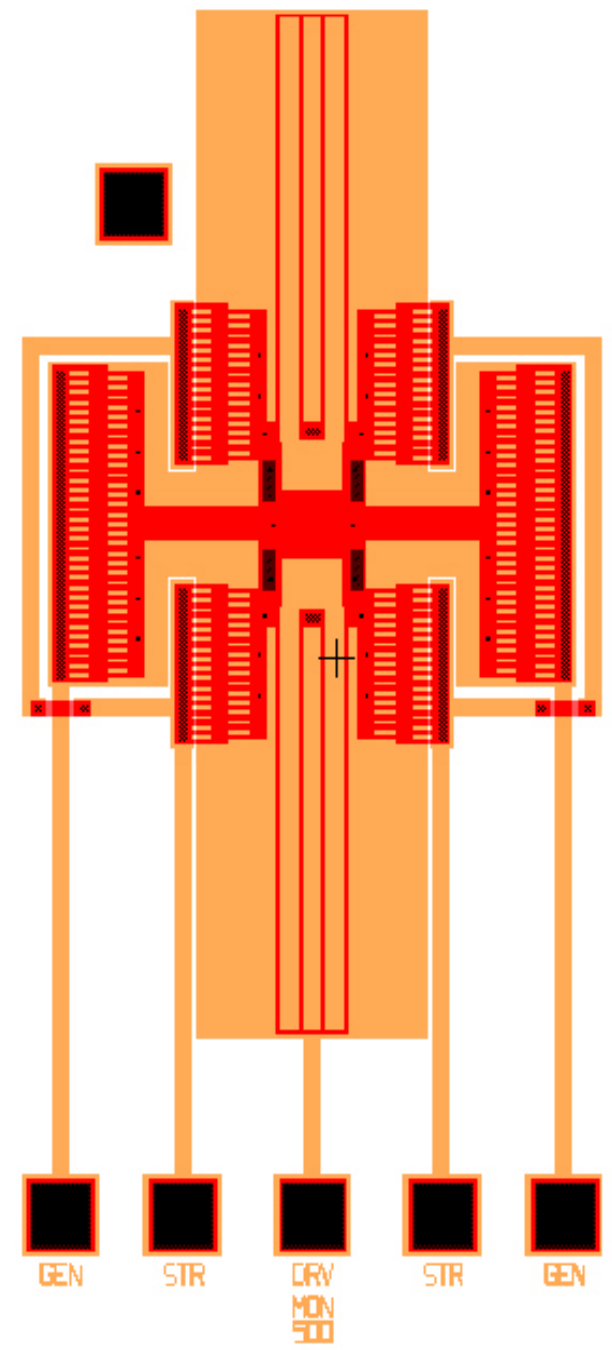

Figure 3.3: Resonator generator geometry.

The final device layout, shown in Figure 3.4, was fabricated by Chronos Integrated Microsystems (formerly MSNC) using their Multi-User MEMS Processing Service (MUMPS). The seven MUMPS mask levels and their cross sections are illustrated in Figures 3.5 - 3.7. Detailed information about the MUMPS process can be found in [60]. 


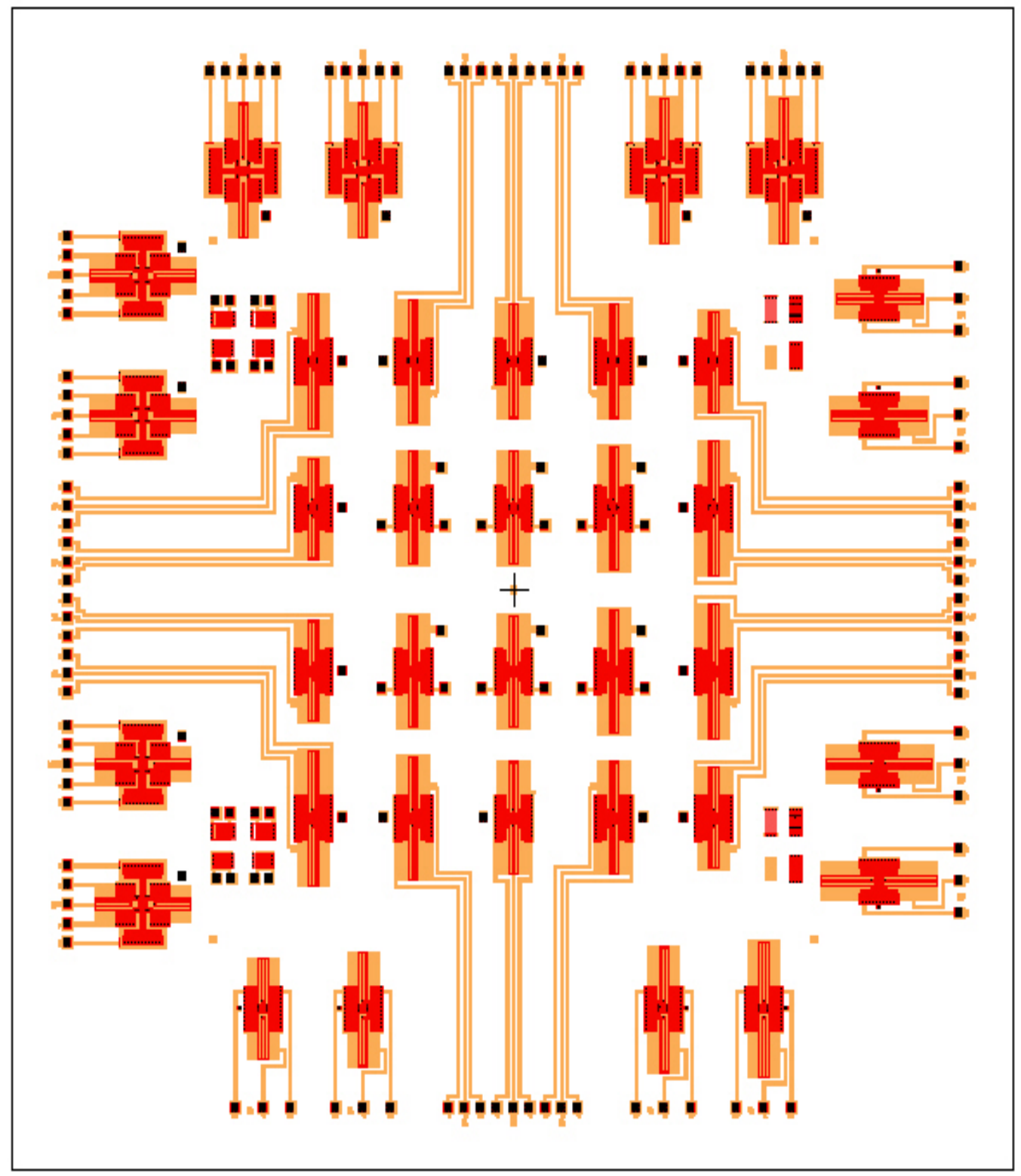

Figure 3.4: MEMS die layout. 


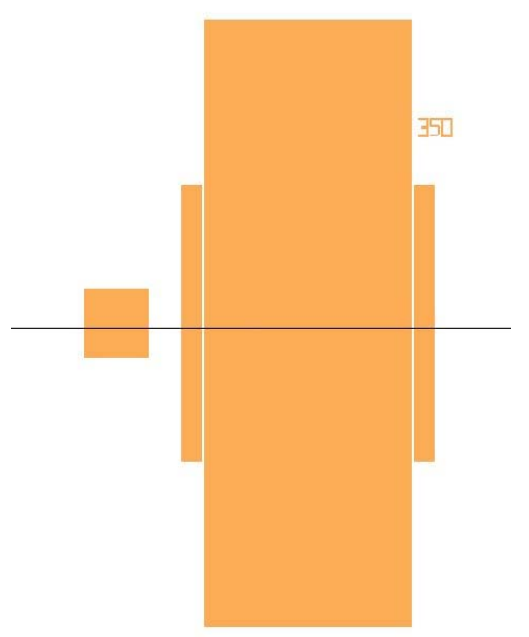

(a)

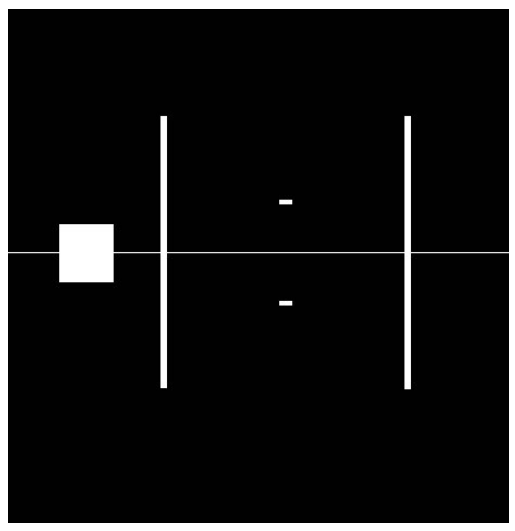

(b)

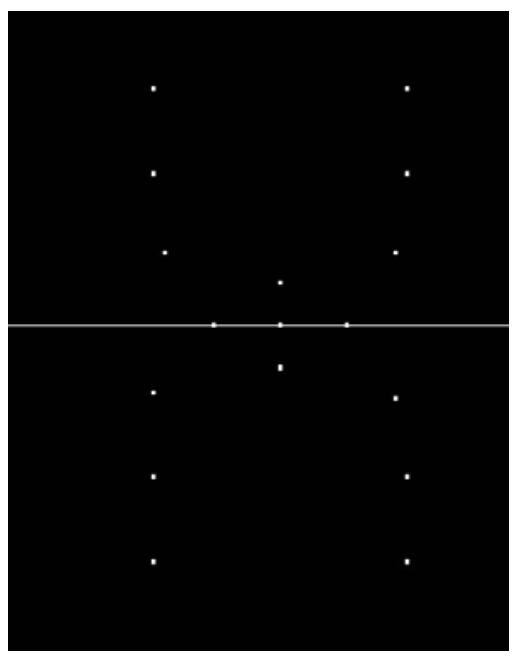

(c)

Figure 3.5: MUMPS mask levels and their corresponding cross sections: (a) POLY 0, (b) ANCHOR 1, and (c) DIMPLE. 
(a)
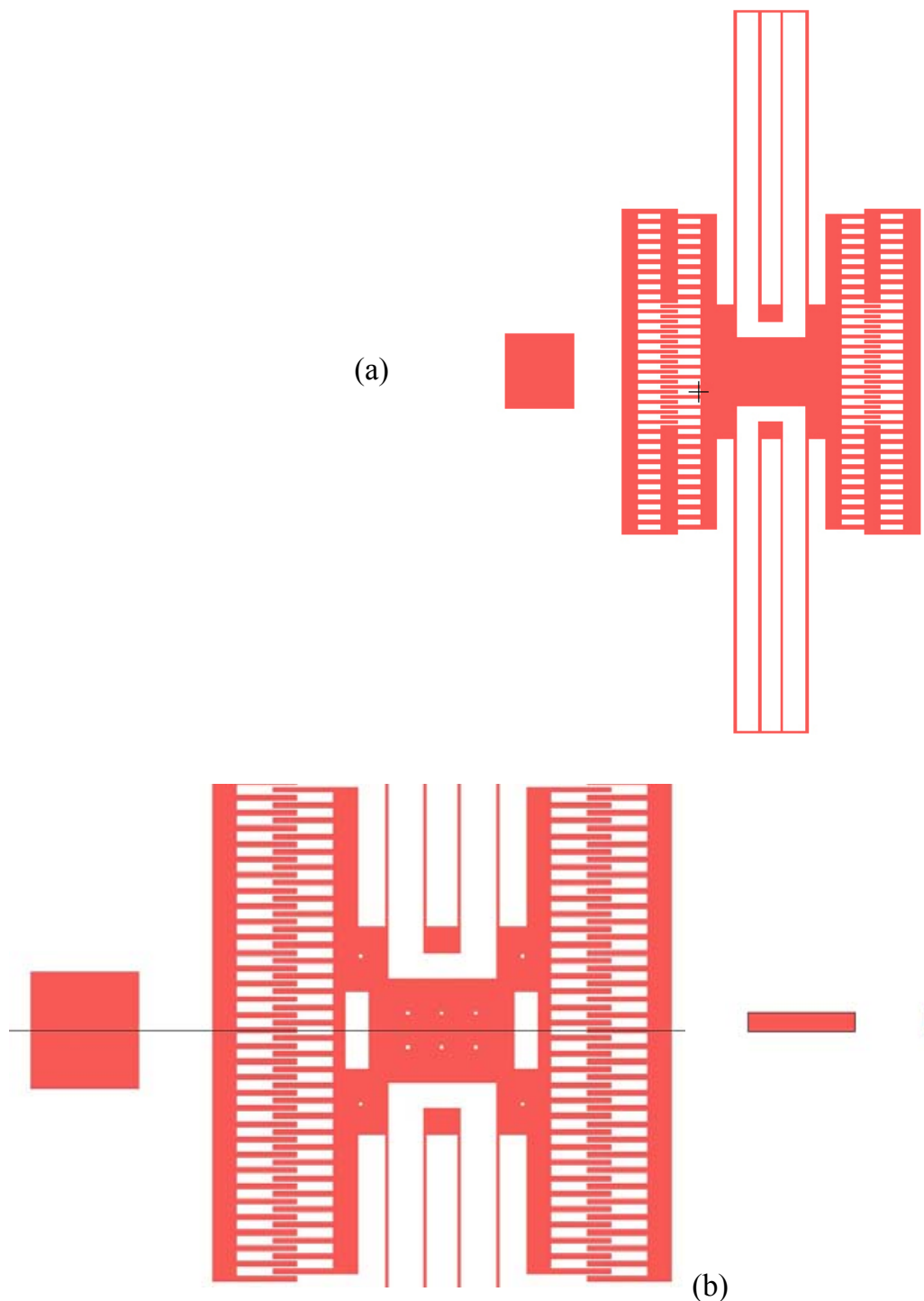

(b)

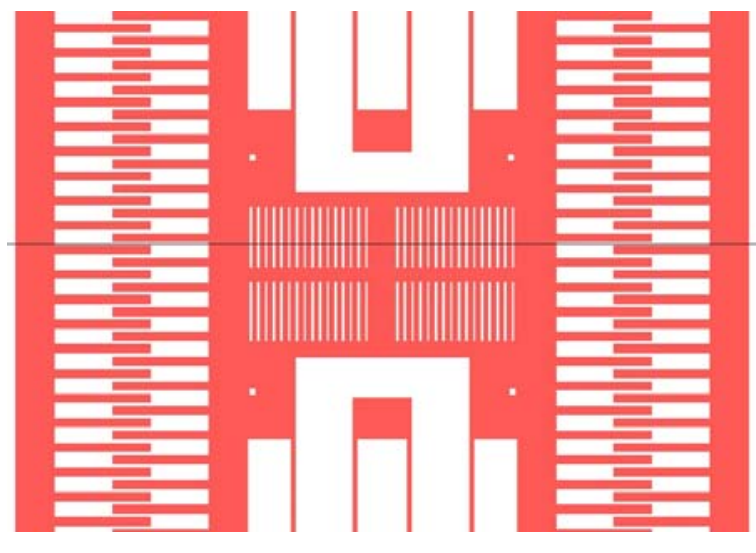

(c)

Figure 3.6: POLY 1 layer: (a) basic device geometry, (b) single opening and cross section, and (c) grating and cross section. 


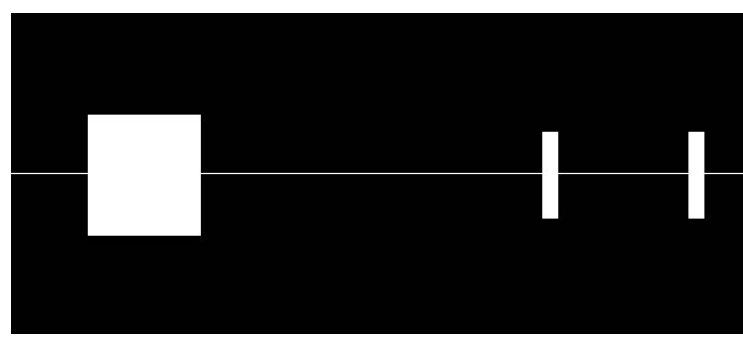

(a)

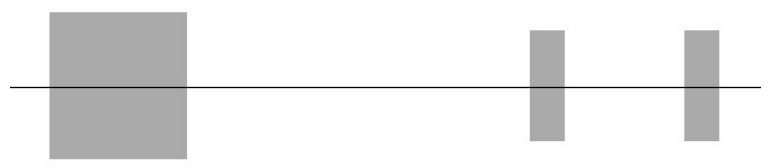

(b)

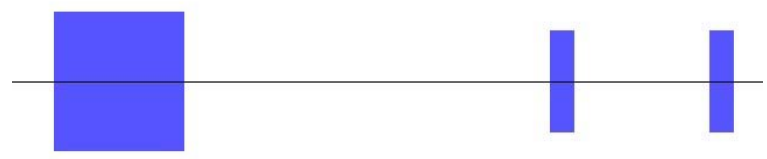

(c)

Figure 3.7: MUMPS mask levels and their corresponding cross sections: (a) POLY 1- POLY 2 VIA, (b) POLY 2, and (c) METAL.

As seen in Figure 3.4, most of the device voltage input pads for the 36 devices were routed to the edge of the die for packaging and bonding to provide more space for the detector fiber in optical probing experiments. A bonding pad (black squares in the layout illustration) was located next to each of the edge-routed devices to confirm the location of the focused probe beam spot and aid in probe beam positioning. As in previous research, each die was back-side polished before sacrificial oxide removal, or release, to improve throughwafer probe signal intensity. After polishing, the die was cleaned thoroughly using acetone and immersed in hydrofluoric acid for 2.5 minutes to release the moving structures of the devices.

To aid in system model development for control purposes, mass and resonant frequency for each type of device (basic resonators and generator structures) was calculated with asdrawn dimension values using Equations 2.1-3 and 2.1-4. Since the MUMPs process has a $\pm 0.3 \mu \mathrm{m}$ linewidth tolerance for $2 \mu \mathrm{m}$ features, high and low values of spring constant and resonant frequency were calculated (because of the cubed dependence on flexure width) to 
determine the range that the experimentally measured resonant frequency could fall within. These results are presented in Tables 3.1, 3.2 and 3.3.

Table 3.1: Theoretical effective mass.

\begin{tabular}{|c|c|c|c|c|c|}
\hline \multirow{3}{*}{$\begin{array}{l}\text { Flexure } \\
\text { Length }\end{array}$} & \multicolumn{5}{|c|}{ Device Mass (kg) } \\
\hline & \multicolumn{3}{|c|}{ Basic Structure } & \multicolumn{2}{|c|}{ Generator Structure } \\
\hline & Grating & POLY 2 & POLY $2 \&$ Metal & Grating & Basic \\
\hline 350 & $1.920 \mathrm{E}-10$ & $2.099 \mathrm{E}-10$ & $2.245 \mathrm{E}-10$ & $4.374 \mathrm{E}-10$ & $4.262 \mathrm{E}-10$ \\
\hline 400 & $1.930 \mathrm{E}-10$ & $2.109 \mathrm{E}-10$ & $2.255 \mathrm{E}-10$ & $4.384 \mathrm{E}-10$ & $4.272 \mathrm{E}-10$ \\
\hline 450 & $1.946 \mathrm{E}-10$ & $2.125 \mathrm{E}-10$ & $2.271 \mathrm{E}-10$ & $4.400 \mathrm{E}-10$ & $4.288 \mathrm{E}-10$ \\
\hline 500 & $1.959 \mathrm{E}-10$ & $2.138 \mathrm{E}-10$ & $2.284 \mathrm{E}-10$ & $4.413 \mathrm{E}-10$ & $4.301 \mathrm{E}-10$ \\
\hline
\end{tabular}

Table 3.2: Theoretical spring constant.

\begin{tabular}{|c|c|c|c|c|c|c|}
\hline \multirow{3}{*}{$\begin{array}{l}\text { Flexure } \\
\text { Length }\end{array}$} & \multicolumn{6}{|c|}{ Spring Constant } \\
\hline & \multirow{2}{*}{$\begin{array}{l}\text { Width }=2 \mu \mathrm{m} \\
\text { Nominal K }\end{array}$} & \multicolumn{2}{|c|}{ Process Error $+/-0.3 \mu \mathrm{m}$} & \multirow{2}{*}{$\begin{array}{c}\text { Width }=2 \mu \mathrm{m} \\
\text { Actual Nominal K }\end{array}$} & \multicolumn{2}{|c|}{ Process Error $+/-0.3 \mu \mathrm{m}$} \\
\hline & & High $\mathrm{K}$ & Low $\mathrm{K}$ & & Actual High K & $\begin{array}{c}\text { Actual Low } \\
\text { K }\end{array}$ \\
\hline 350 & 0.12315 & 0.18729 & 0.07563 & 0.11576 & 0.17606 & 0.07109 \\
\hline 400 & 0.08250 & 0.12547 & 0.05067 & 0.07692 & 0.11699 & 0.04724 \\
\hline 450 & 0.05794 & 0.08812 & 0.03558 & 0.05360 & 0.08151 & 0.03291 \\
\hline 500 & 0.04224 & 0.06424 & 0.02594 & 0.03877 & 0.05897 & 0.02381 \\
\hline
\end{tabular}


Table 3.3: Resonant frequency.

\begin{tabular}{||c||c|c|c||c|c|c||}
\hline \multirow{2}{*}{$\begin{array}{c}\text { Flexure } \\
\text { Length }\end{array}$} & \multicolumn{2}{c|}{ Resonance (Hz): Basic - Grating } & \multicolumn{3}{c||}{ Resonance (Hz): Basic - POLY 2 } \\
\cline { 2 - 7 } & Low & Nominal & High & Low & Nominal & High \\
\hline \hline 350 & 3062 & 3908 & 4819 & 2929 & 3738 & 4609 \\
\hline 400 & 2490 & 3177 & 3918 & 2382 & 3039 & 3748 \\
\hline 450 & 2070 & 2641 & 3257 & 1981 & 2528 & 3117 \\
\hline 500 & 1755 & 2239 & 2761 & 1680 & 2143 & 2643 \\
\hline
\end{tabular}

\begin{tabular}{||c||c|c|c||c|c|c||}
\hline \multirow{2}{*}{$\begin{array}{c}\text { Flexure } \\
\text { Length }\end{array}$} & \multicolumn{2}{c||}{ Resonance (Hz): Basic - POLY 2 \& Metal } & \multicolumn{3}{c||}{ Resonance (Hz): Generator - Grating } \\
\cline { 2 - 7 } & Low & Nominal & High & Low & Nominal & High \\
\hline \hline 350 & 2832 & 3614 & 4457 & 2029 & 2589 & 3193 \\
\hline 400 & 2304 & 2939 & 3625 & 1652 & 2108 & 2600 \\
\hline 450 & 1916 & 2445 & 3015 & 1376 & 1757 & 2166 \\
\hline 500 & 1625 & 2074 & 2557 & 1169 & 1492 & 1840 \\
\hline
\end{tabular}

\begin{tabular}{|c||c|c|c||}
\hline \multirow{2}{*}{$\begin{array}{c}\text { Flexure } \\
\text { Length }\end{array}$} & \multicolumn{3}{|c||}{ Resonance (Hz): Generator - Basic } \\
\cline { 2 - 4 } & Low & Nominal & High \\
\hline \hline 350 & 2055 & 2623 & 3235 \\
\hline 400 & 1674 & 2136 & 2634 \\
\hline 450 & 1394 & 1779 & 2194 \\
\hline 500 & 1184 & 1511 & 1864 \\
\hline
\end{tabular}

\subsection{Through-Wafer Probe System Improvement}

Although the previous through-wafer probing setup was constructed on an air damping table, preliminary optical monitoring and control experiments were often plagued by external vibrations due to the instability of the components in the setup itself. To reduce unwanted vibrations, the setup was reconstructed using sturdier, more compatible components. A custom die package, consisting of a modified bottomless chip package mounted on a glass slide, was created to eliminate the need for probes to deliver drive voltages. These probes often obstructed adjustment of the detector fiber in the previous setup. One probe platform was included in the redesign to allow for mechanical actuation of the stages after release to eliminate residual stiction. The addition of motorized translation stages for fine $(0.5 \mu \mathrm{m})$ adjustment of the die allowed for better positioning of the infrared probe spot, resulting in cleaner optical output signals. Figures 3.8 - 3.10 illustrate the probe setup and key components. 


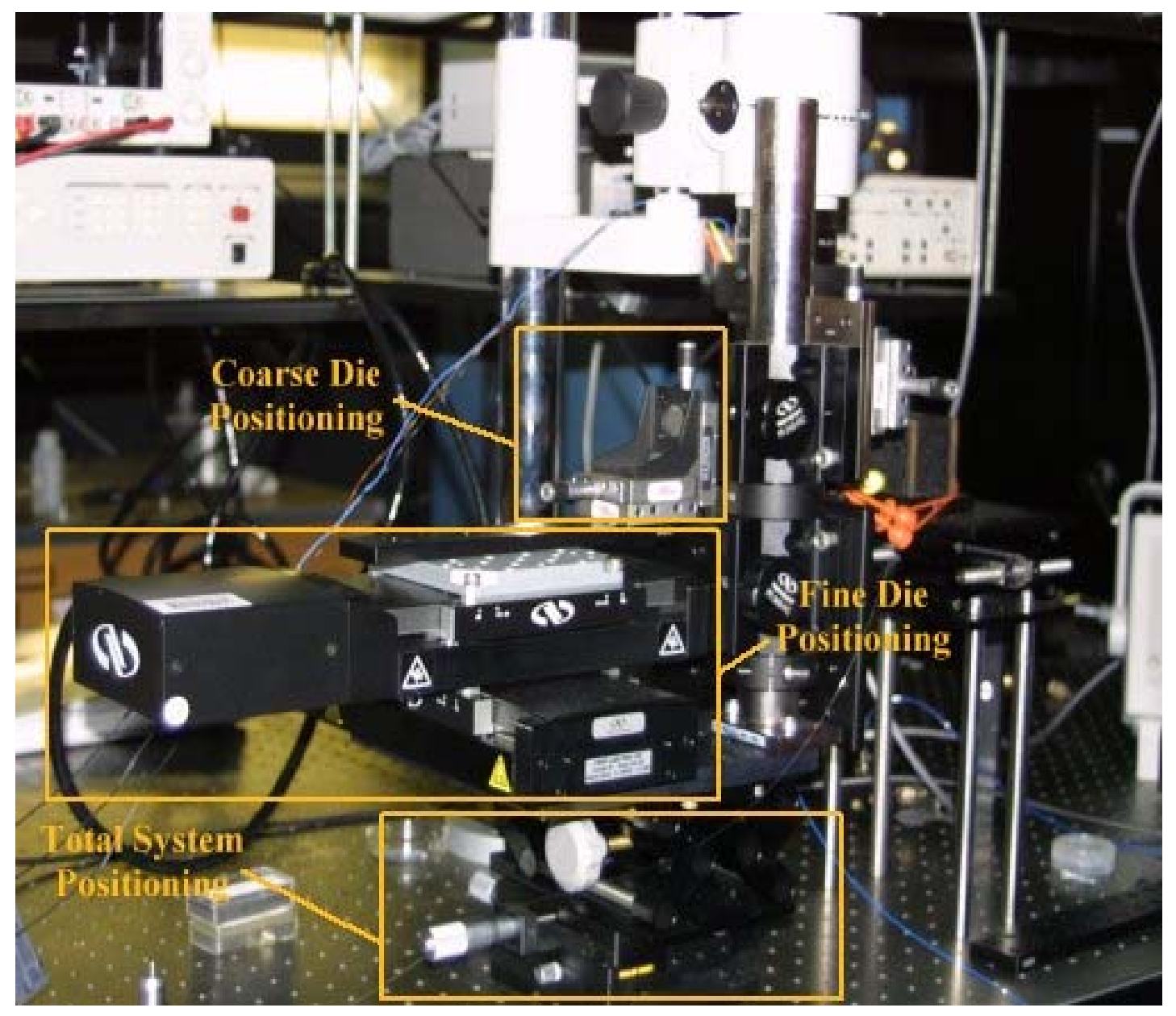

Figure 3.8: Die and total system positioning elements. 


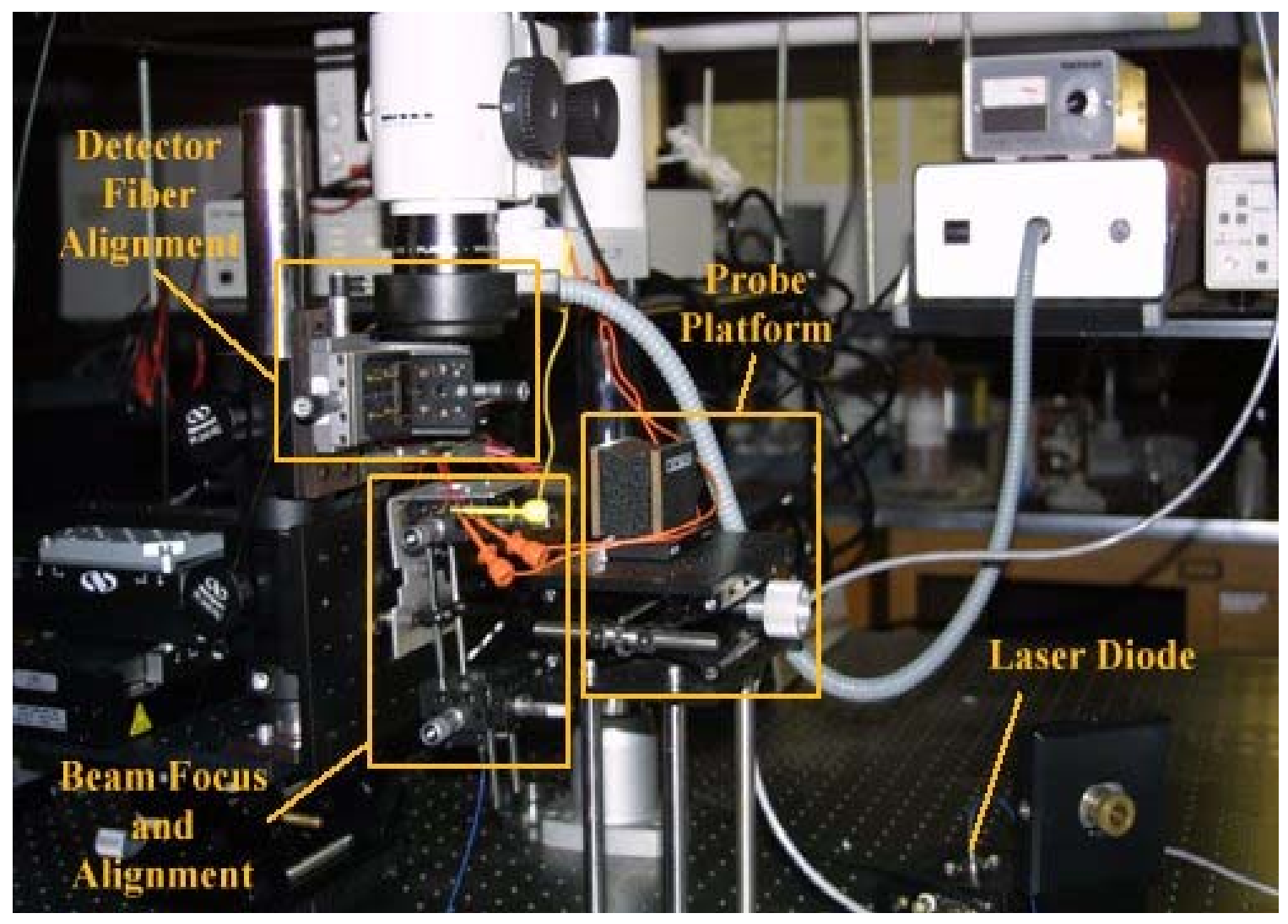

Figure 3.9: Probe beam and detector fiber adjustment. 


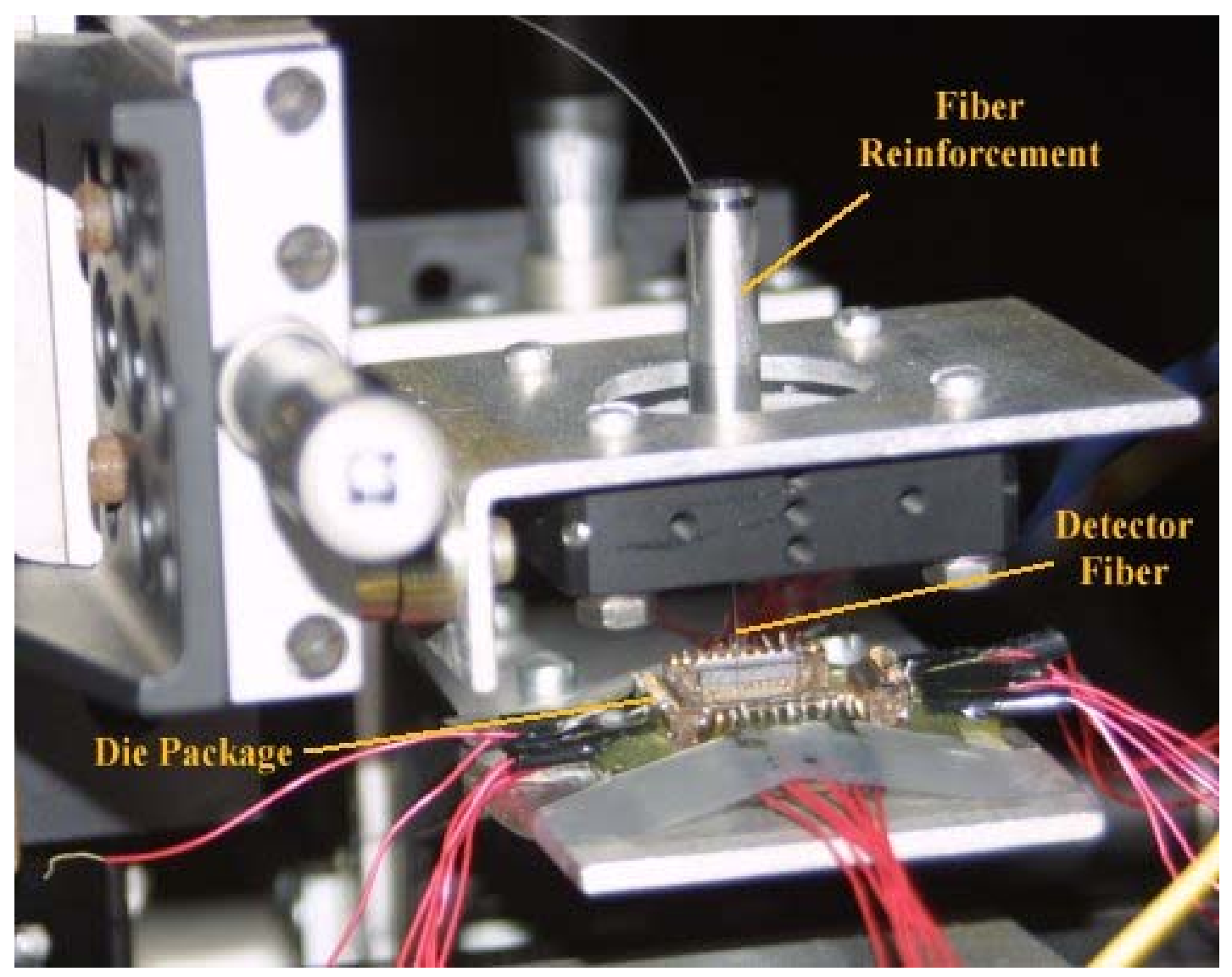

Figure 3.10: Close-up of die package and detector fiber.

As in the previous through-wafer monitoring setup, each major component was given independent three-axis motion for maximum fine tuning and adjustment capability. The dual lens spot focusing arrangement was also improved with the addition of 3-axis adjustment for each lens and by replacing the second GRIN lens in Figure 1.5(c) with a bulk optical lens. These changes allowed for more control over spot size and shape. 


\section{Chapter 4 Through-Wafer Diffraction Study}

This chapter presents the results of using theoretical optical field intensity determination theory presented in Chapter 2 to develop a software tool that can be used to examine the optical field intensity of the trough-wafer probe beam. Simple examples of a slit and rectangular opening are presented to determine the effectiveness of the Matlab simulation programs, allowing more complex stage geometries to be examined. Next, single-axis optical output waveform simulations are generated using the optical field determination program and the second-order forced harmonic oscillator system model with experimentally determined mass, spring constant, and damping parameters (for displacement determination). This simulated output is compared to actual through-wafer optical intensity signals to determine the effectiveness of the simulation and system model. The effects of vertical stage motion, as well as off-axis device probing on the output probe signal are also examined.

\subsection{Plane Wave Diffraction by a Slit}

To begin, a simple plane wave incident on a slit was examined. A Matlab program, found in Appendix A ('planeslit.m'), was developed that performed the convolution of the aperture function of a plane wave incident on a slit $20 \mu \mathrm{m}$ wide $(-10 \mu \mathrm{m}$ to $+10 \mu \mathrm{m})$ in the $x$ direction and infinitely long in the $y$-direction with the transfer function of free space. It used the Fresnel number input by the user to determine observation plane distance. The convolution was only performed in one direction to decrease the complexity of the program, 
but had no adverse effects on the output since the pupil function only varied along the $x$ dimension. Figures $4.1-4.7$ show the slit transmission profile, the aperture shadowed beam pattern, and the resulting output beam intensities for Fresnel numbers of 90, 10, 1, 0.5, and 0.1 .

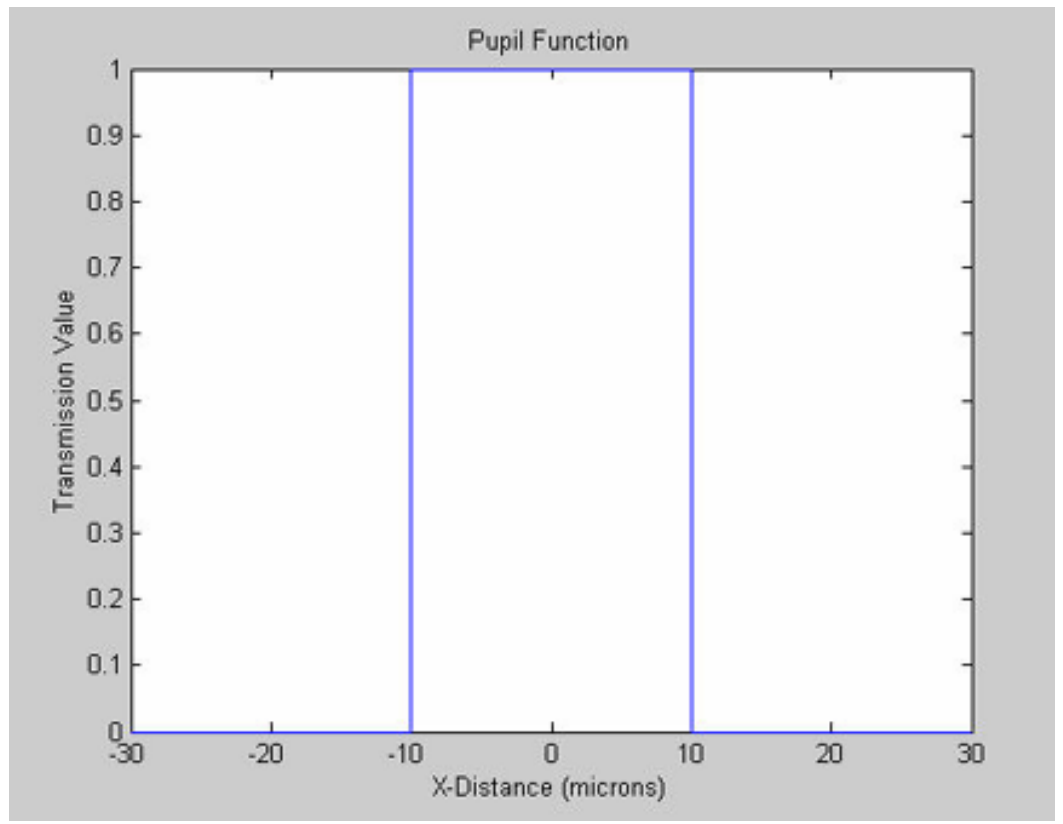

Figure 4.1: Slit transmission profile.

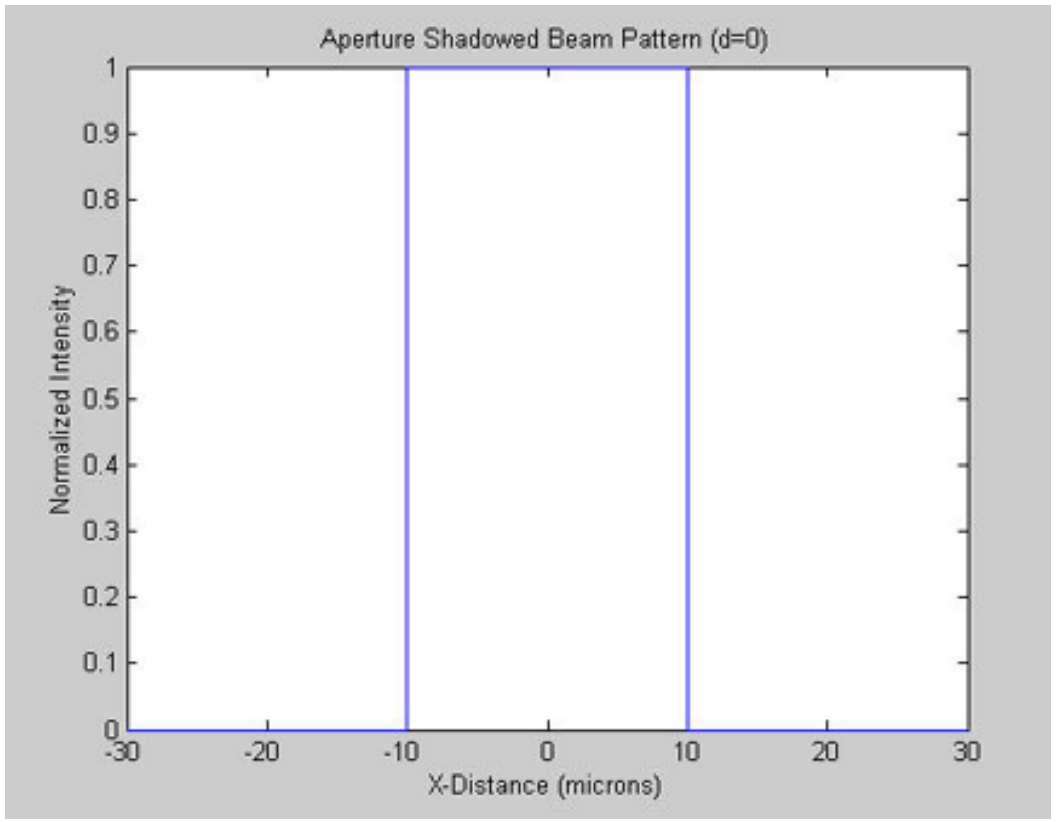

Figure 4.2: Aperture shadowed beam pattern. 


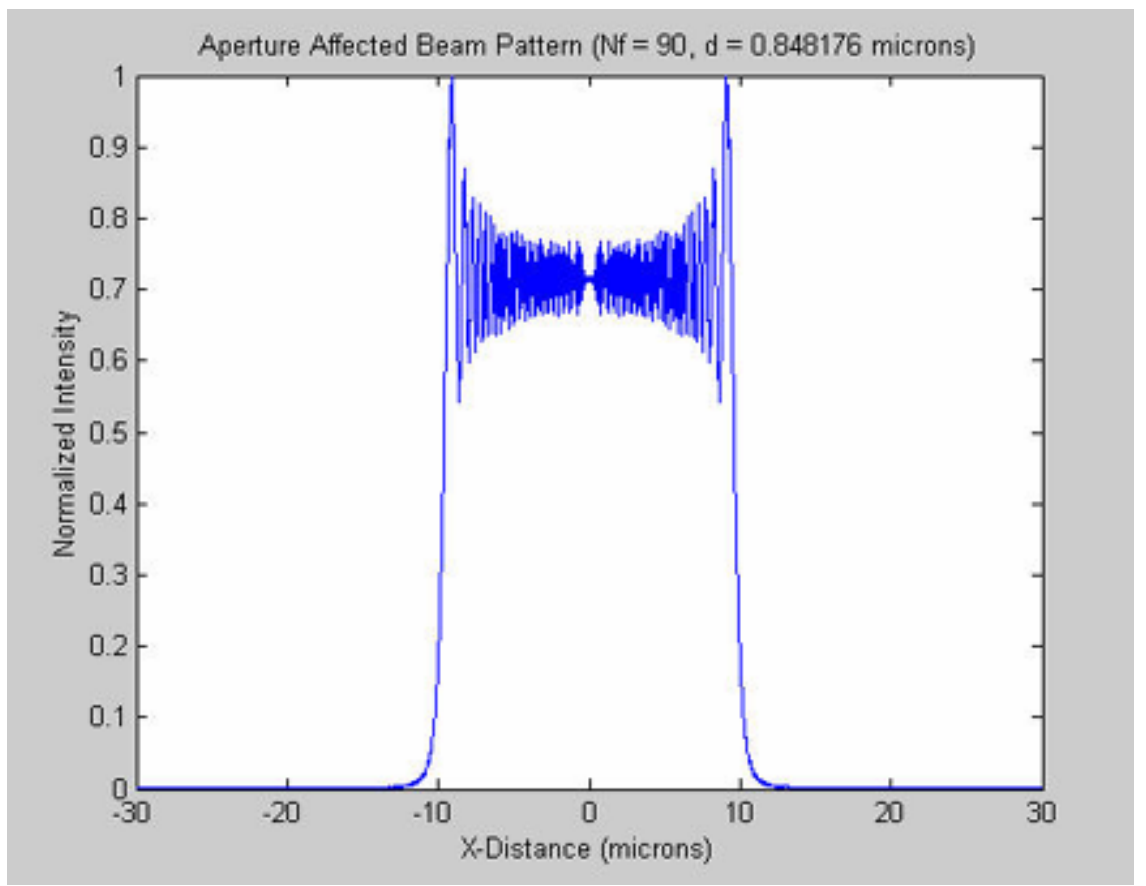

Figure 4.3: Slit effected output beam intensity profile, $\mathrm{N}_{\mathrm{F}}=90, \mathrm{~d}=0.85 \mu \mathrm{m}$.

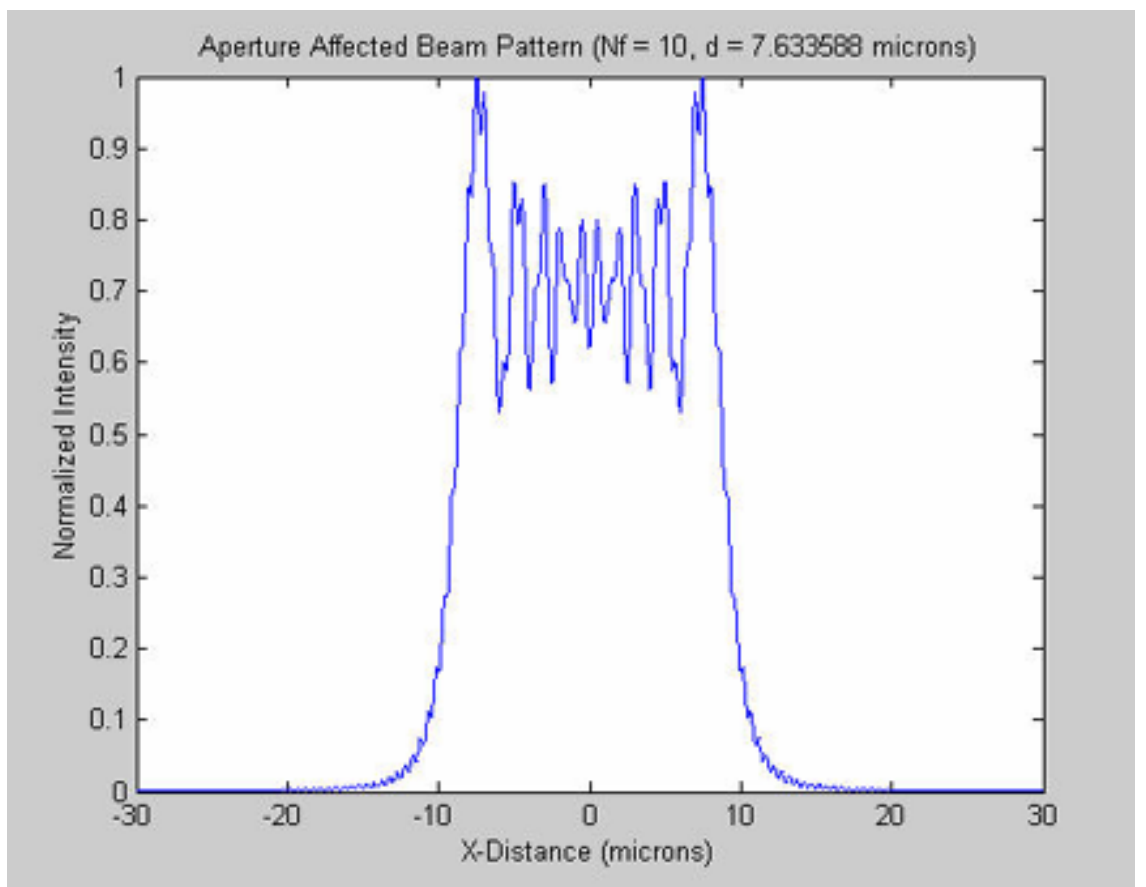

Figure 4.4: Slit effected output beam intensity profile, $\mathrm{N}_{\mathrm{F}}=10, \mathrm{~d}=7.63 \mu \mathrm{m}$. 


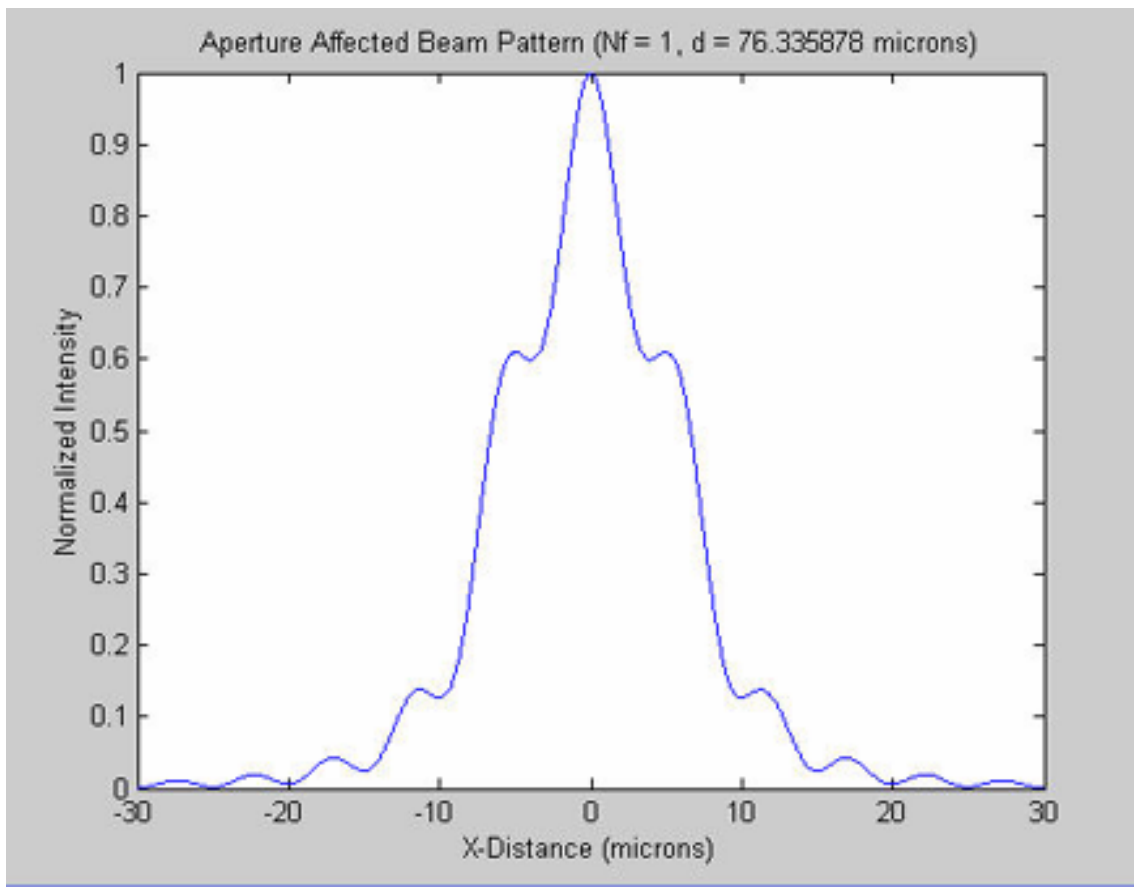

Figure 4.5: Slit effected output beam intensity profile, $\mathrm{N}_{\mathrm{F}}=1, \mathrm{~d}=76.33 \mu \mathrm{m}$.

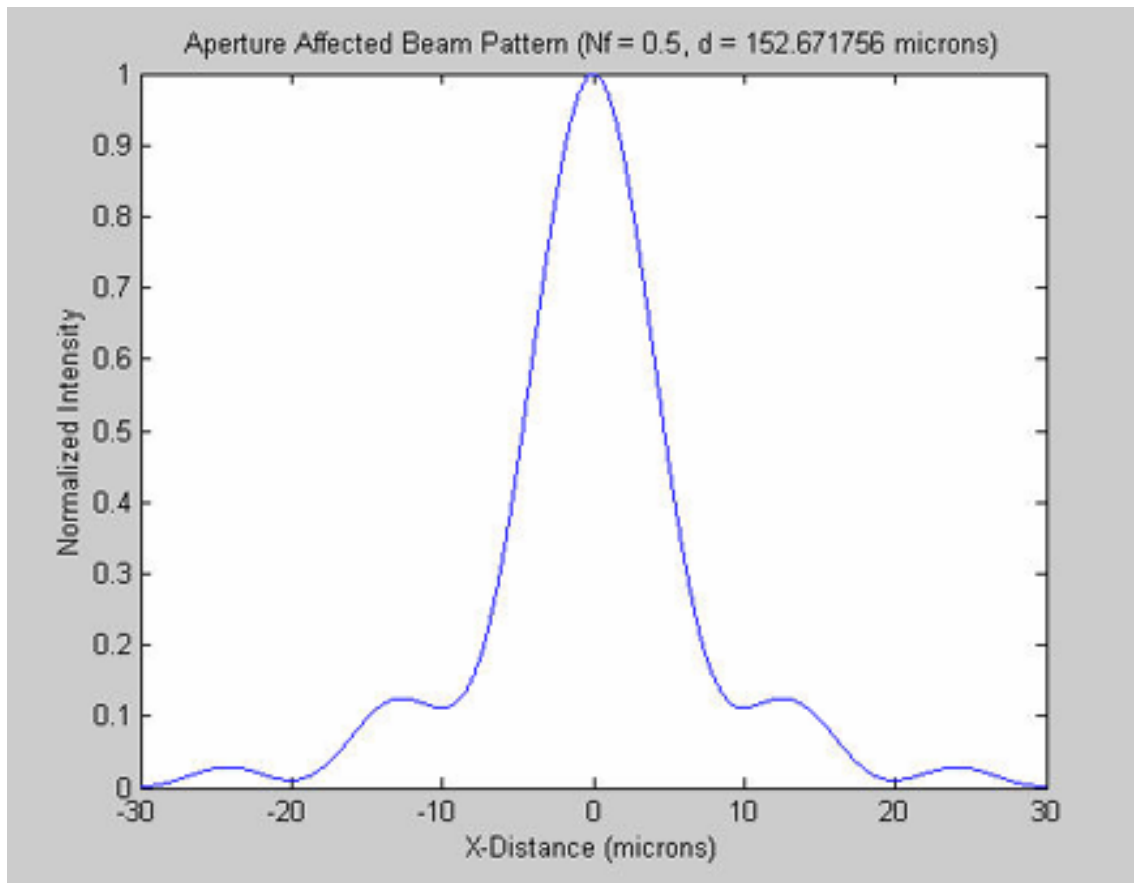

Figure 4.6: Slit effected output beam intensity profile, $\mathrm{N}_{\mathrm{F}}=0.5, \mathrm{~d}=152.67 \mu \mathrm{m}$. 


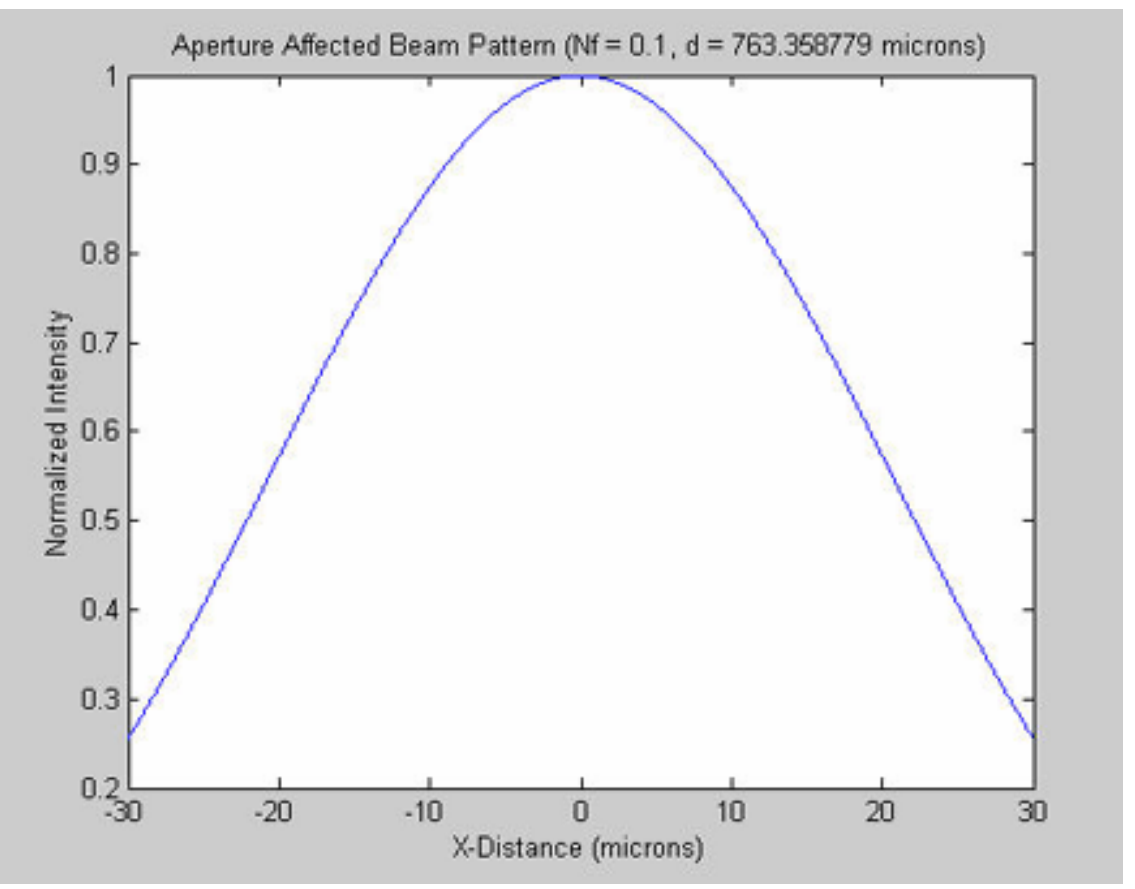

Figure 4.7: Slit effected output beam intensity profile, $\mathrm{N}_{\mathrm{F}}=0.5, \mathrm{~d}=76.33 \mu \mathrm{m}$.

It should be mentioned that certain parameters of the Matlab program have an effect on the output. If the arrays or matricies making up $U(r), p(x, y)$, and $h(x, y)$ do not contain enough zeros, or if the step size of the calculation loop is not small enough, there will be unnatural peaks in the output plane beam intensity at low Fresnel numbers or multiple intensity patterns at high Fresnel numbers, as shown in Figure 4.8 (a) \& (b).

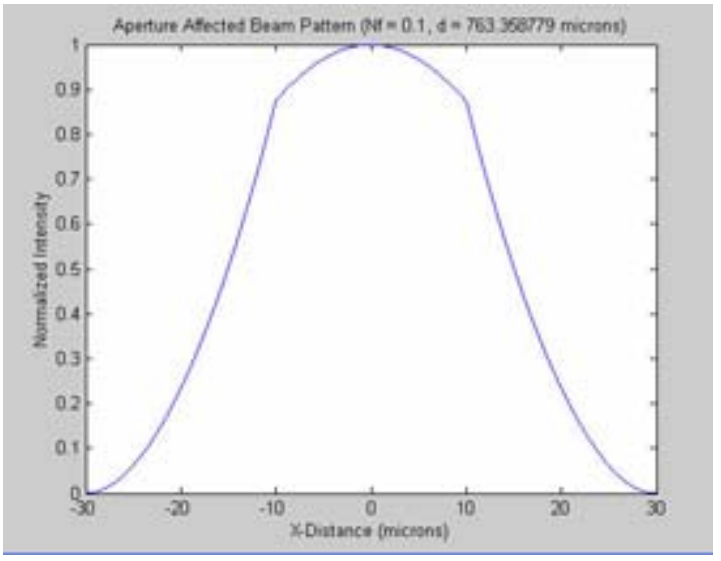

(a)

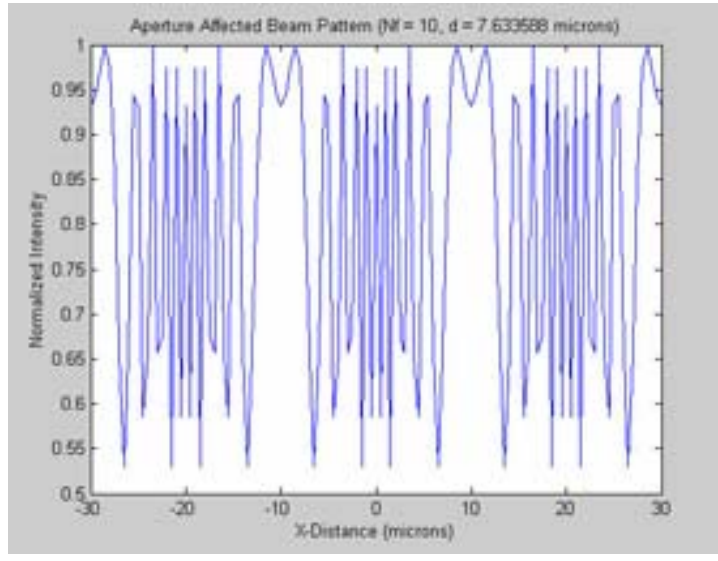

(b)

Figure 4.8: Program output errors: (a) $\mathrm{N}_{\mathrm{F}}=0.1$, not enough zeros included in arrays for convolution (b) $\mathrm{N}_{\mathrm{F}}=10$, calculation loop step size too small. 
These effects were thought to be a result of the transfer function of free space being a Fresnel approximation, but were later eliminated by increasing the zero padding and decreasing the computational iteration.

Figure 4.2 shows the aperture shadowed beam pattern, which is the beam intensity at a distance of zero. At the Fresnel number is decreased (corresponding to an increase in distance from zero, and eventually a transition from the Fresnel diffraction region to the Fraunhofer diffraction region), diffraction effects can be seen clearly, causing multiple intensity peaks and the spreading of the beam intensity over a larger area. This result follows exactly the examination performed in [50].

\subsection{Plane Wave Diffraction by a Rectangular Aperture}

The beam intensity pattern after passing through a rectangular aperture $10 \mu \mathrm{m}$ by $30 \mu \mathrm{m}$ was examined by adding another dimension to the functions in a new Matlab program (Appendix A, 'planerect.m') written for the study of slit diffraction. Due to the small step size and large matrices needed to overcome computational anomalies (shown in Figure 4.8), only $\mathrm{N}_{\mathrm{F}}=1,0.5,0.25,0.1$, and 0.05 were simulated. The results are illustrated in Figures 4.9 -4.14 . 


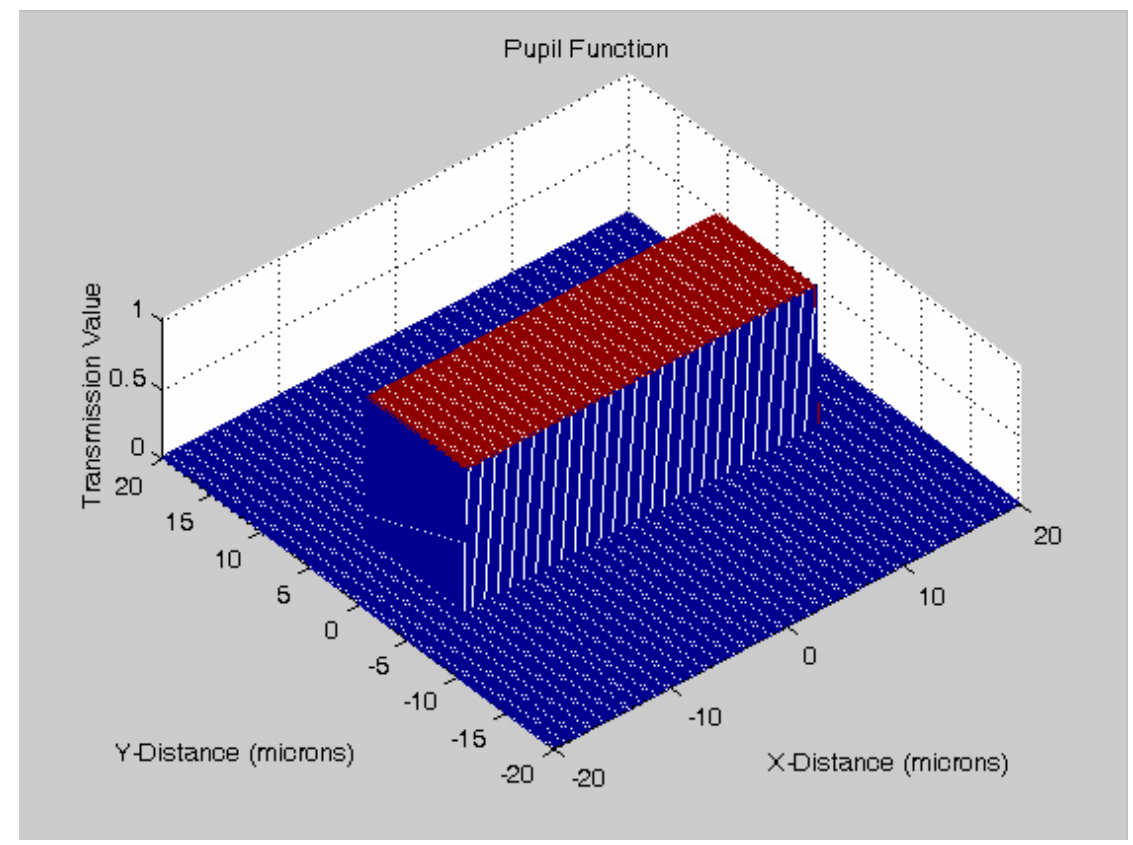

(a)

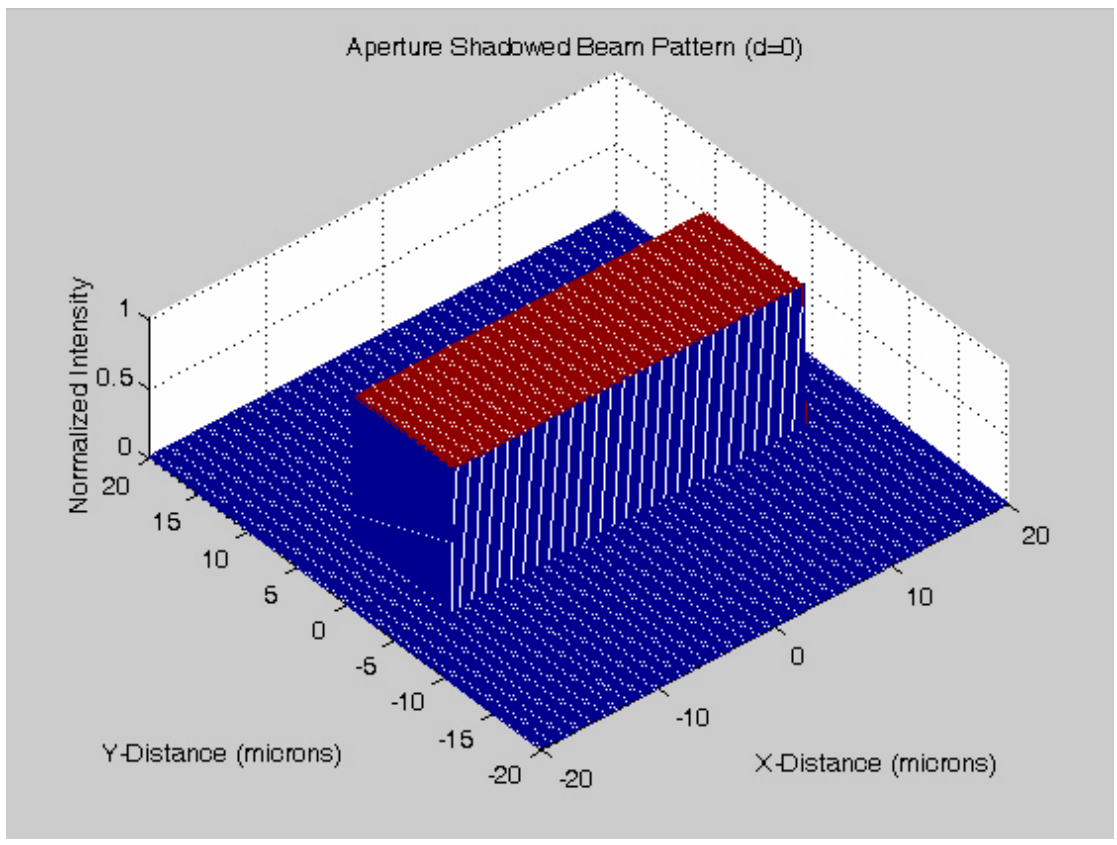

(b)

Figure 4.9: Rectangular aperture pupil function (a) and its corresponding aperture shadowed beam pattern (b). 


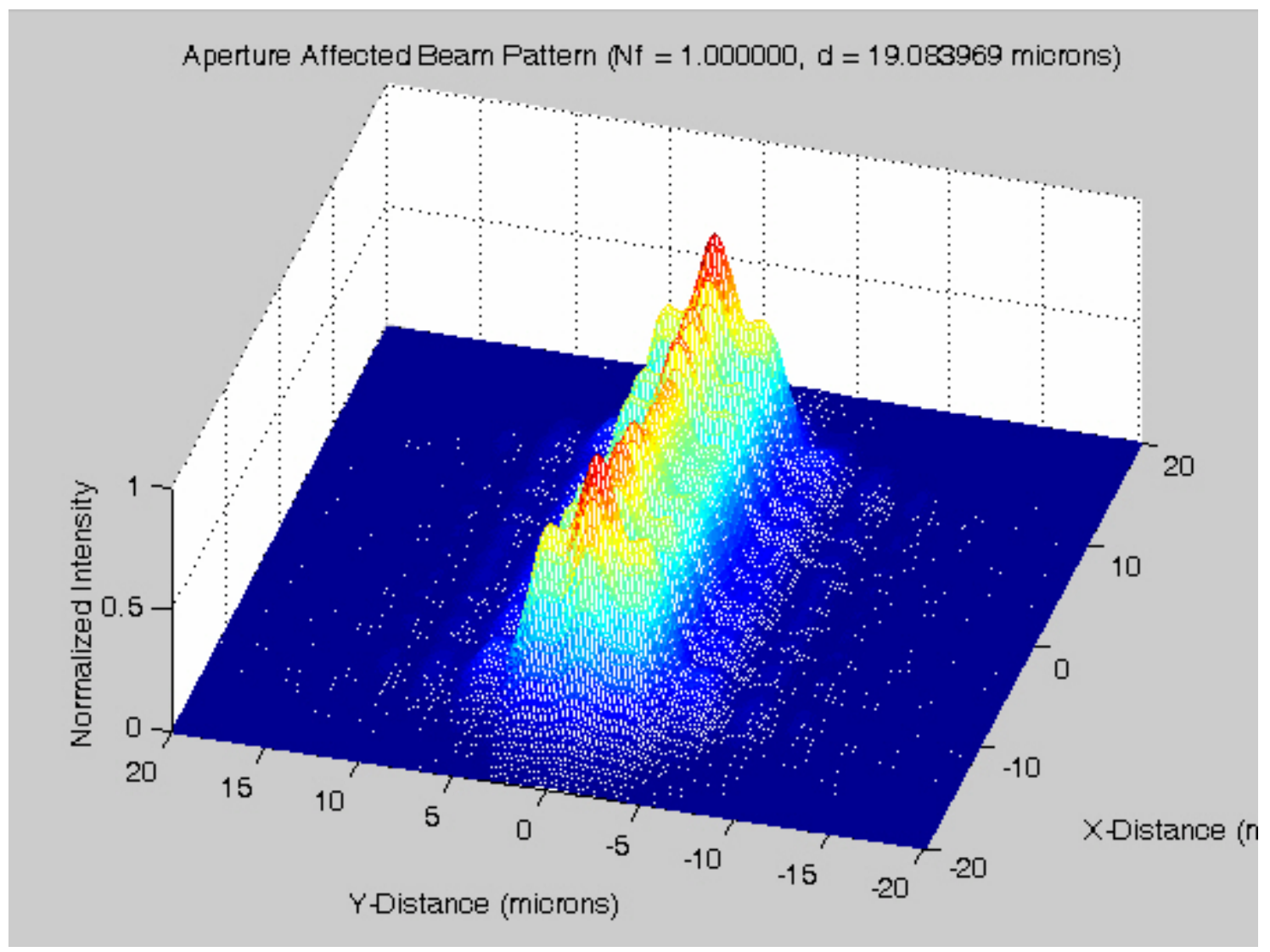

(a)

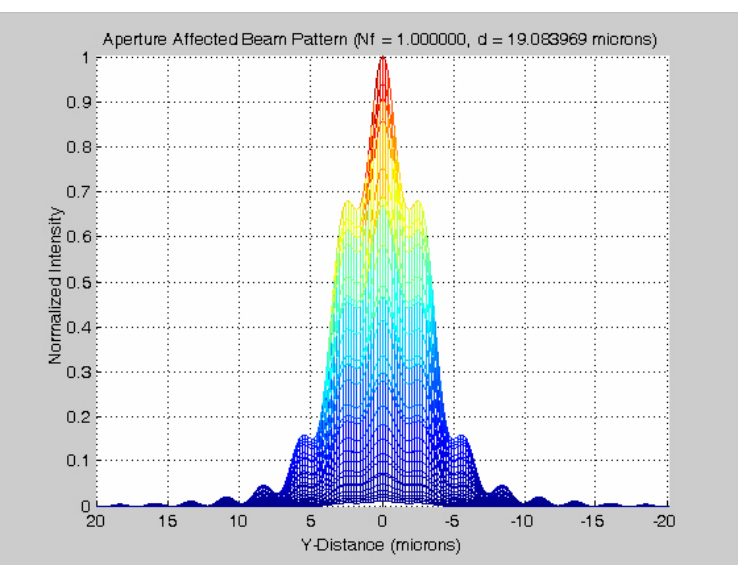

(b)

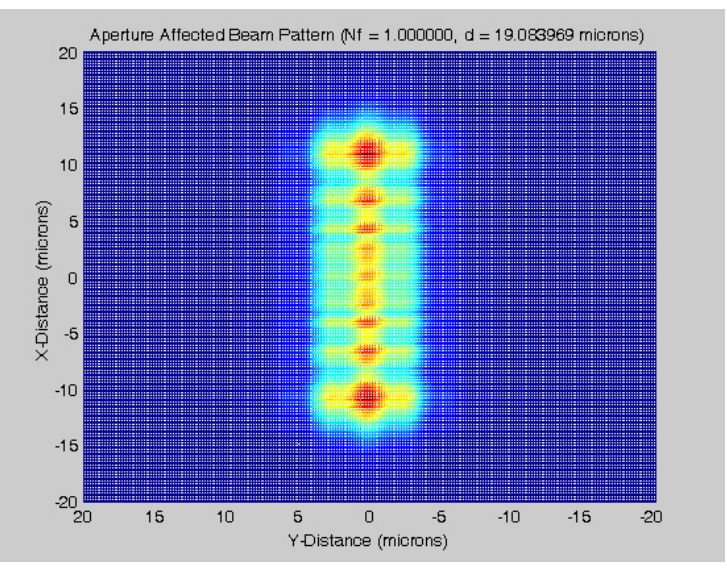

(c)

Figure 4.10: Beam intensity at $\mathrm{N}_{\mathrm{F}}=1, \mathrm{~d}=19 \mu \mathrm{m}$ (a), side view (b), and top view (c). 


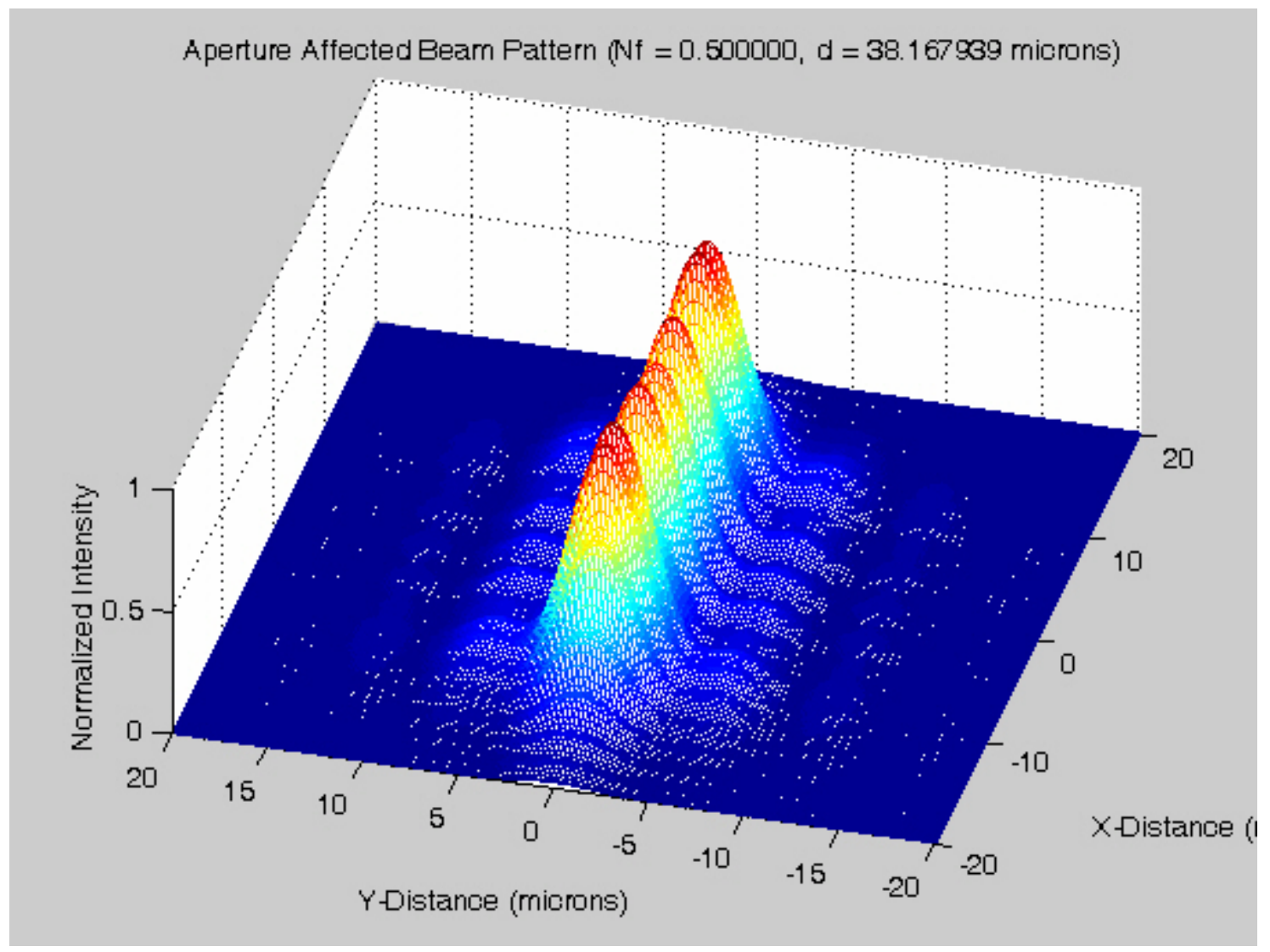

(a)

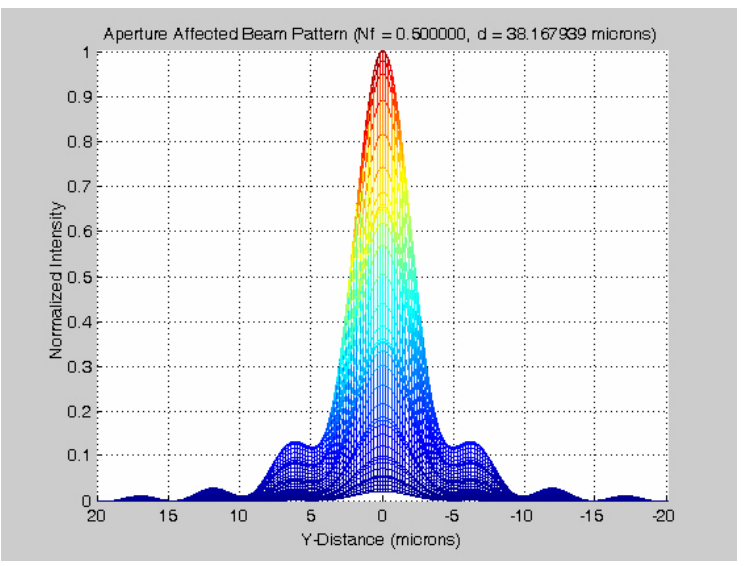

(b)

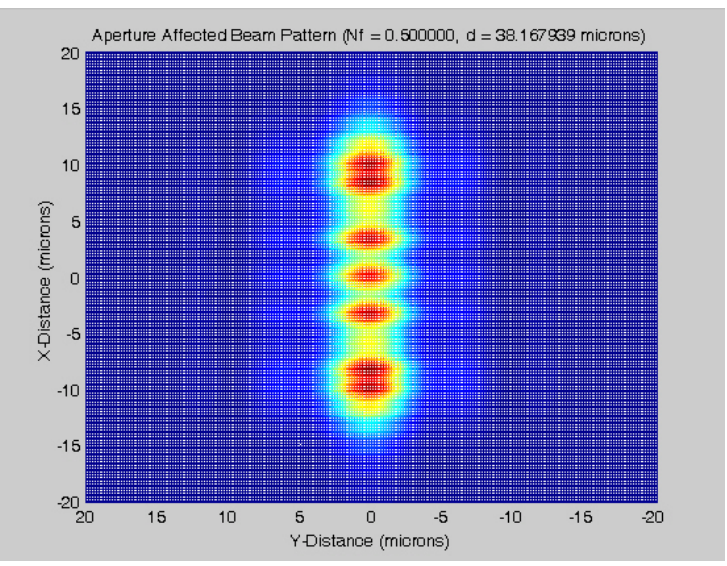

(c)

Figure 4.11: Beam intensity at $\mathrm{N}_{\mathrm{F}}=0.5, \mathrm{~d}=38.2 \mu \mathrm{m}$ (a), side view (b), and top view (c). 


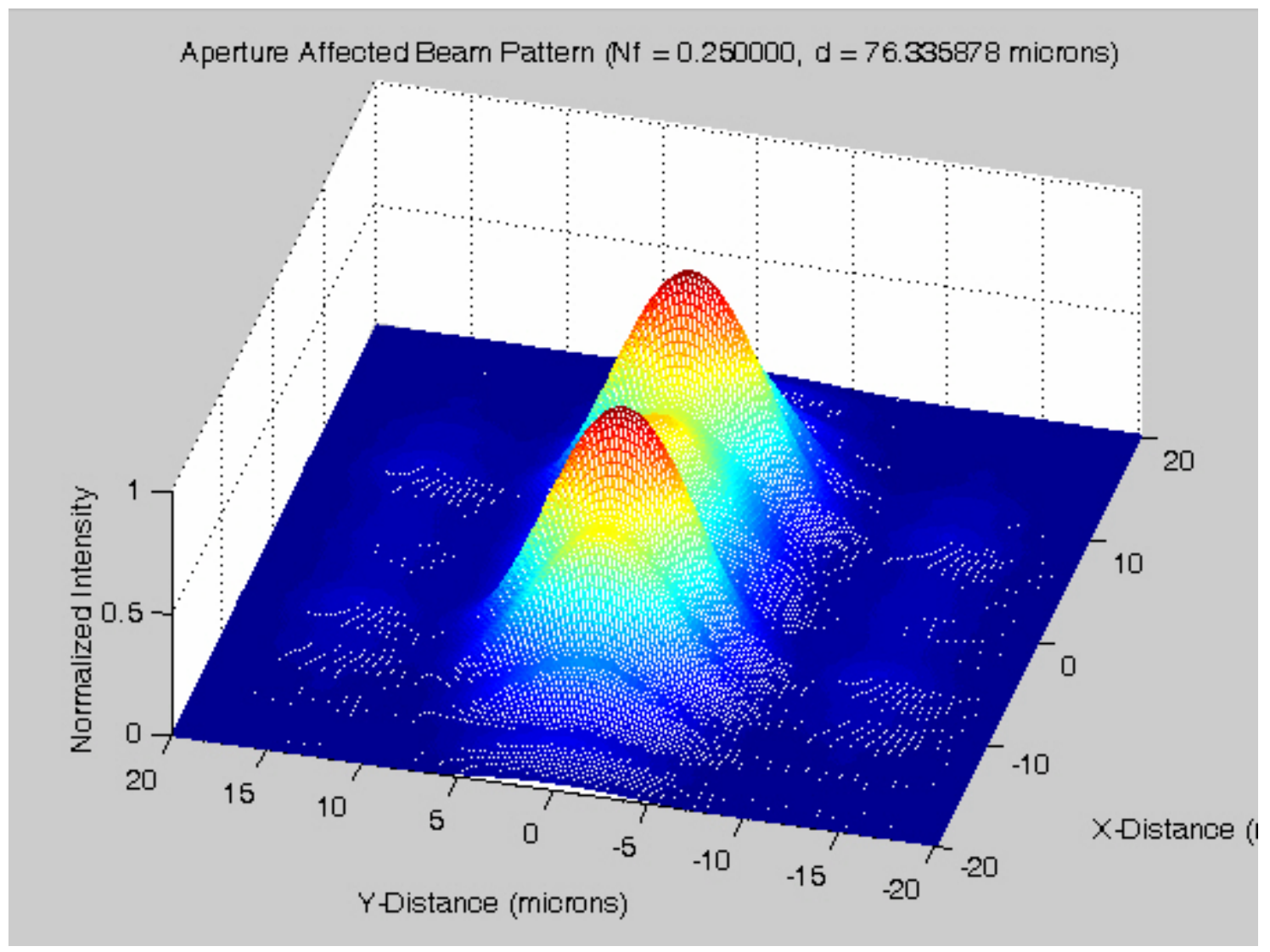

(a)

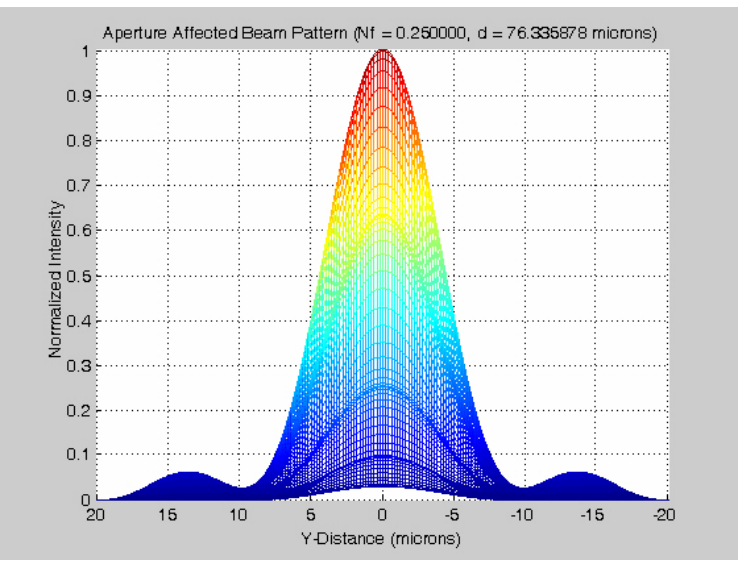

(b)

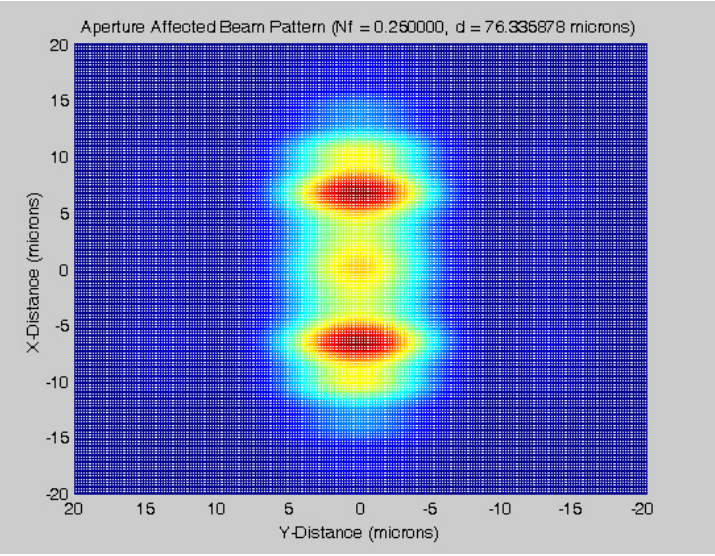

(c)

Figure 4.12: Beam intensity at $\mathrm{N}_{\mathrm{F}}=0.25, \mathrm{~d}=76.3 \mu \mathrm{m}$ (a), side view (b), and top view (c). 


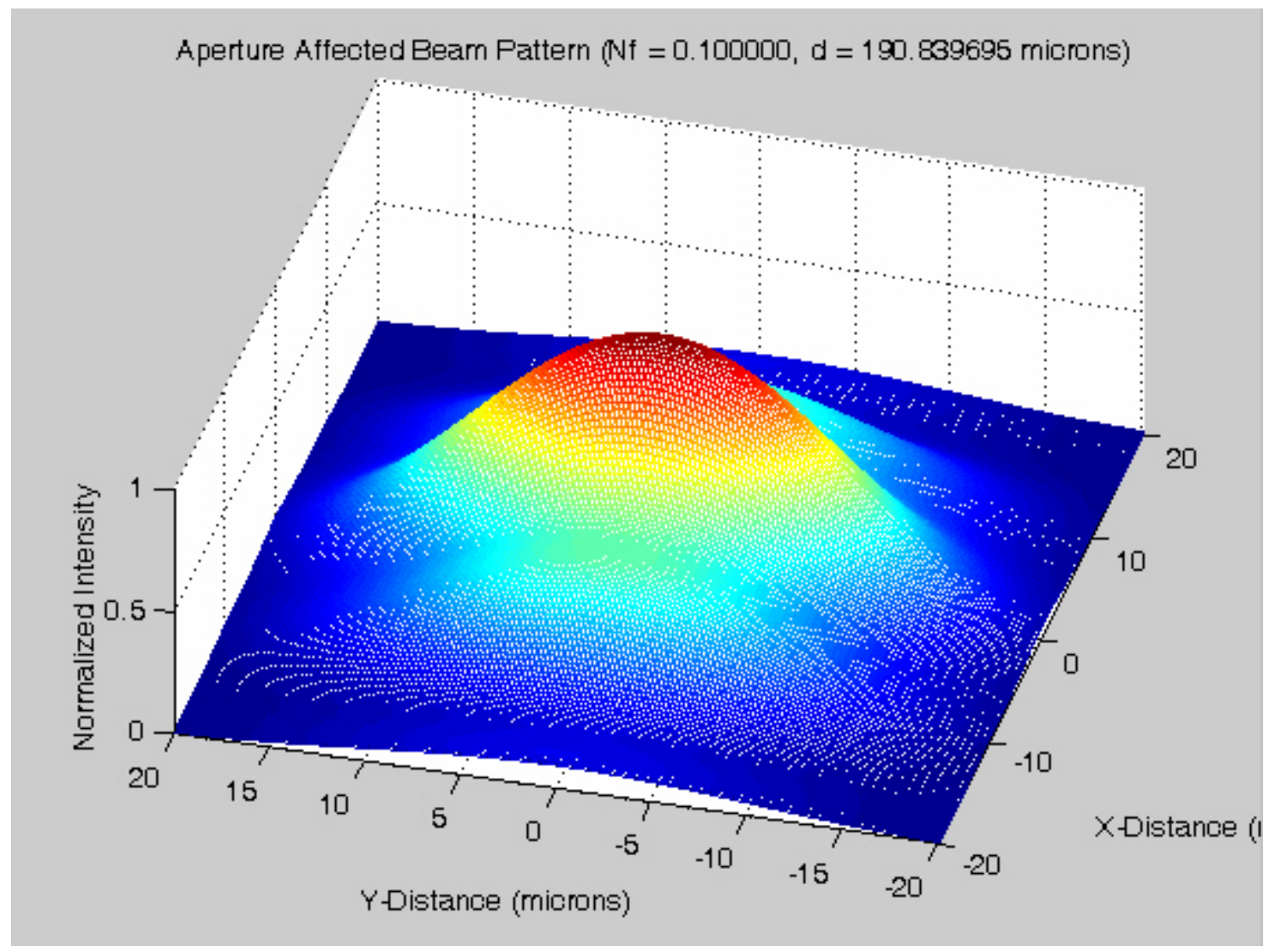

(a)

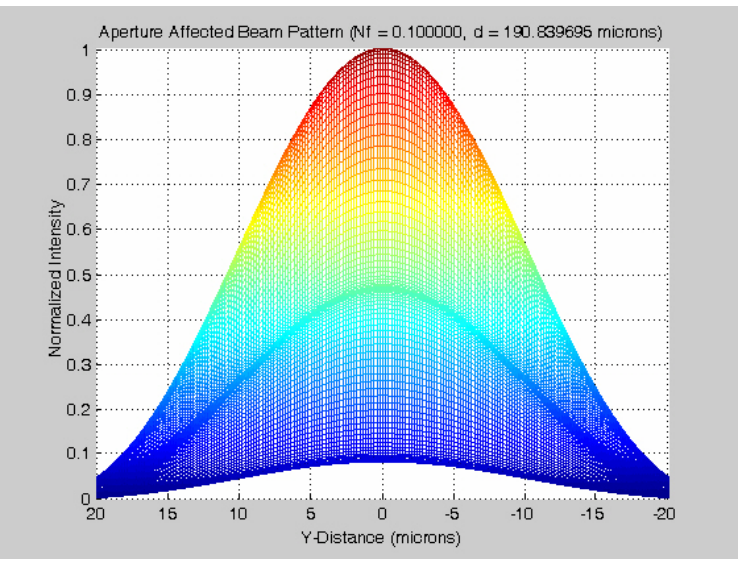

(b)

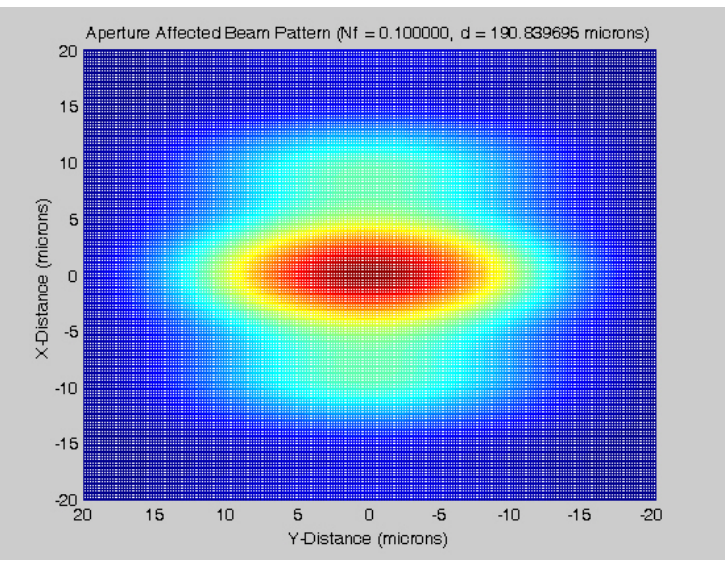

(c)

Figure 4.13: Beam intensity at $\mathrm{N}_{\mathrm{F}}=0.1, \mathrm{~d}=190.8 \mu \mathrm{m}$ (a), side view (b), and top view (c). 


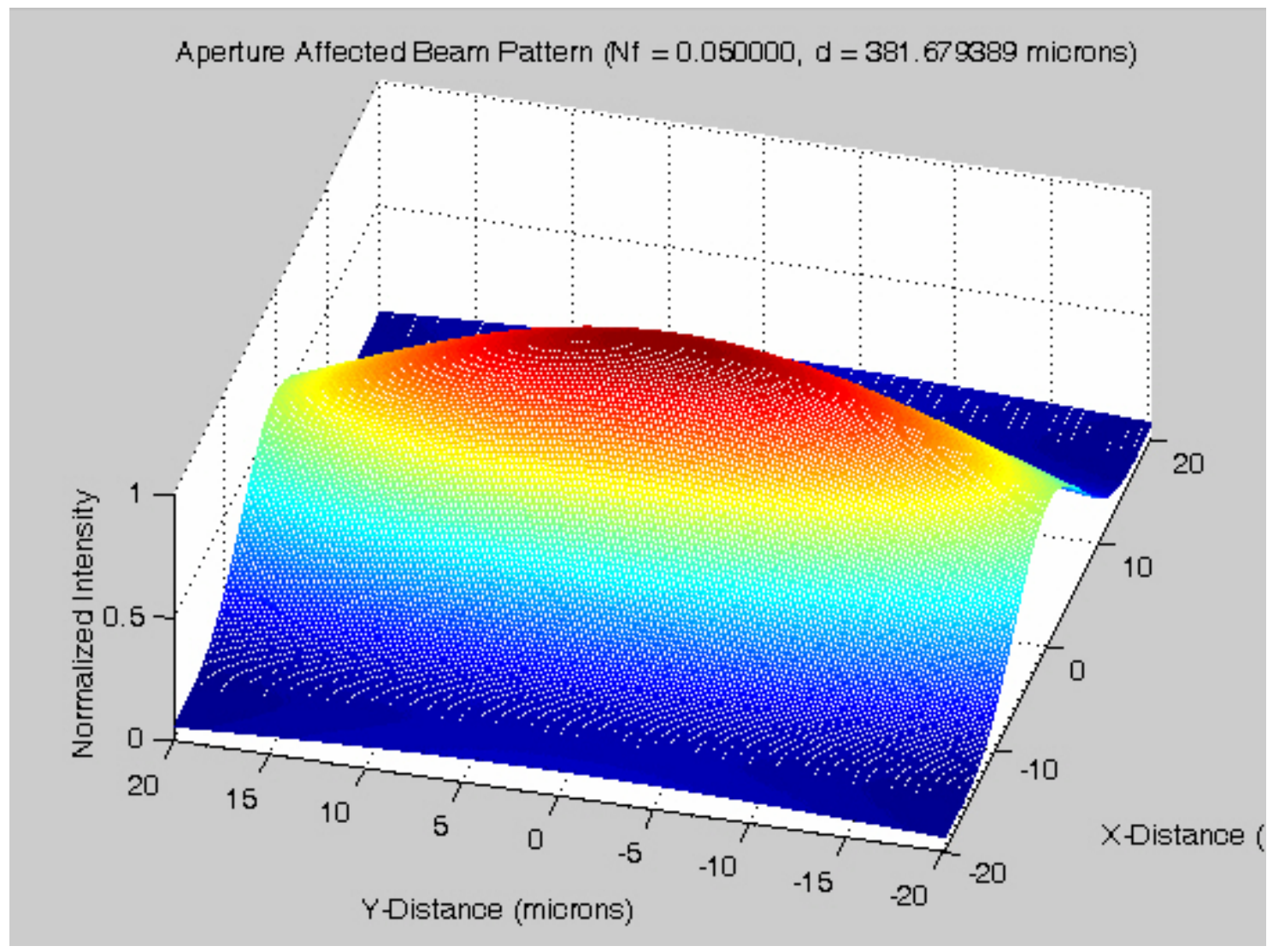

(a)

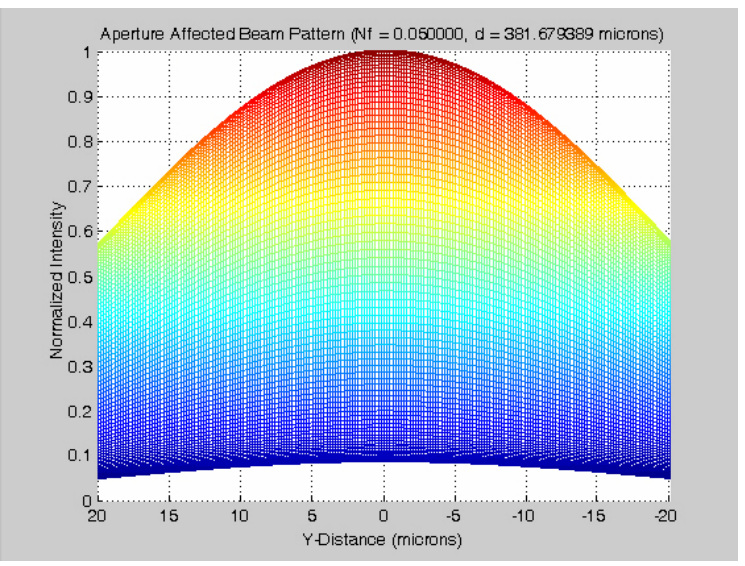

(b)

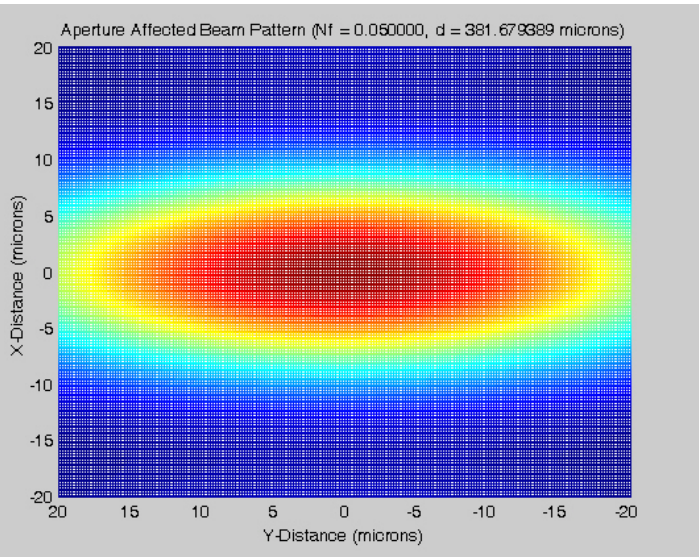

(c)

Figure 4.14: Beam intensity at $\mathrm{N}_{\mathrm{F}}=0.05, \mathrm{~d}=381.7 \mu \mathrm{m}$ (a), side view (b), and top view (c). 
It can be seen that these results follow those of the slit examination when viewed from the side. Visible in the top views, the beam also exhibits diffraction effects in the $\mathrm{x}$-direction as well due to the dimensionality of the aperture.

\subsection{Plane Wave Diffraction Through a Grating}

To determine the effectiveness of using Matlab to simulate diffraction in more complicated structures, a plane wave diffraction through a periodic grating was examined next (Appendix A, 'planegrate.m'). The grating was defined as $2 \mu \mathrm{m}$ openings separated by 2 $\mu \mathrm{m}$ opaque regions. Fresnel numbers of 90, 10, 1, 0.5, and 0.1 were examined, with the results presented in Figures 4.15 - 4.19. The pupil function and aperture shadowed beam pattern are similar to that of a single slit, except they are periodic in nature.

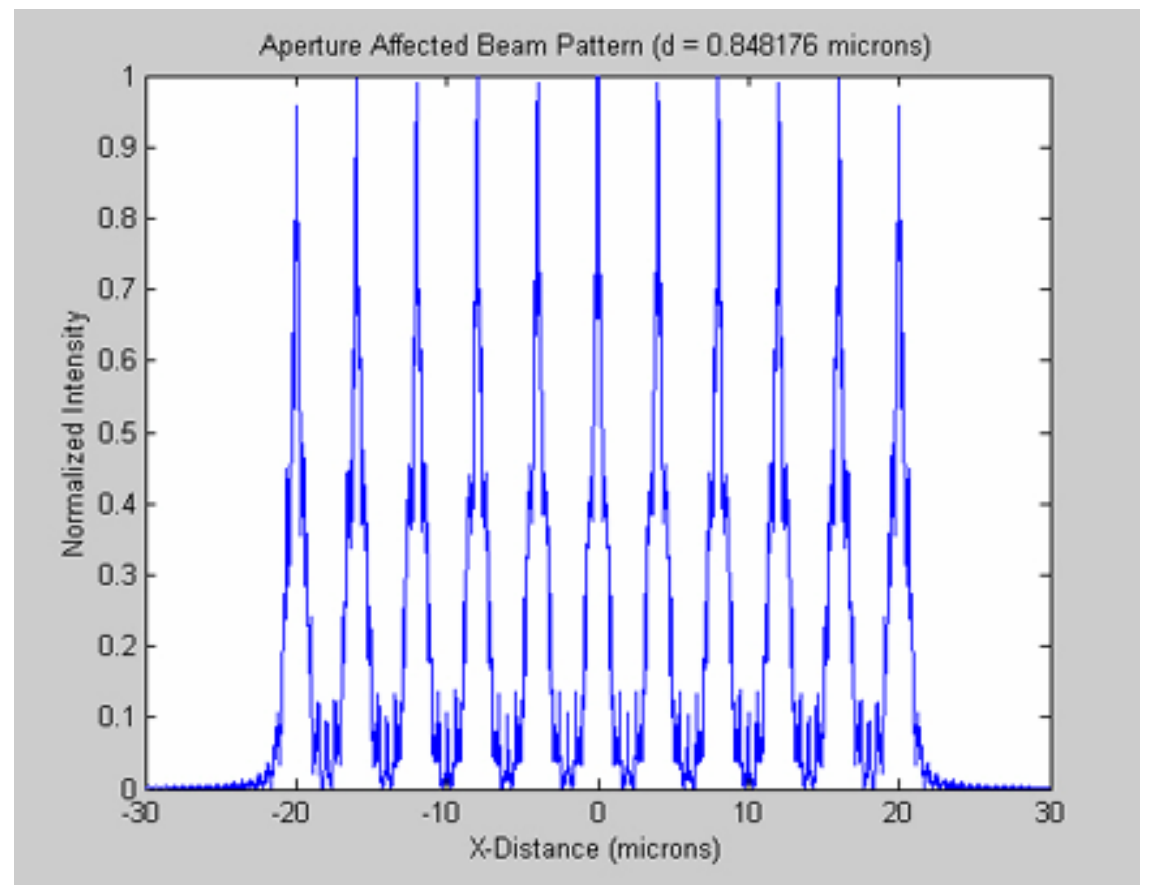

Figure 4.15: Grating effected output beam intensity profile, $\mathrm{N}_{\mathrm{F}}=90, \mathrm{~d}=0.85 \mu \mathrm{m}$. 


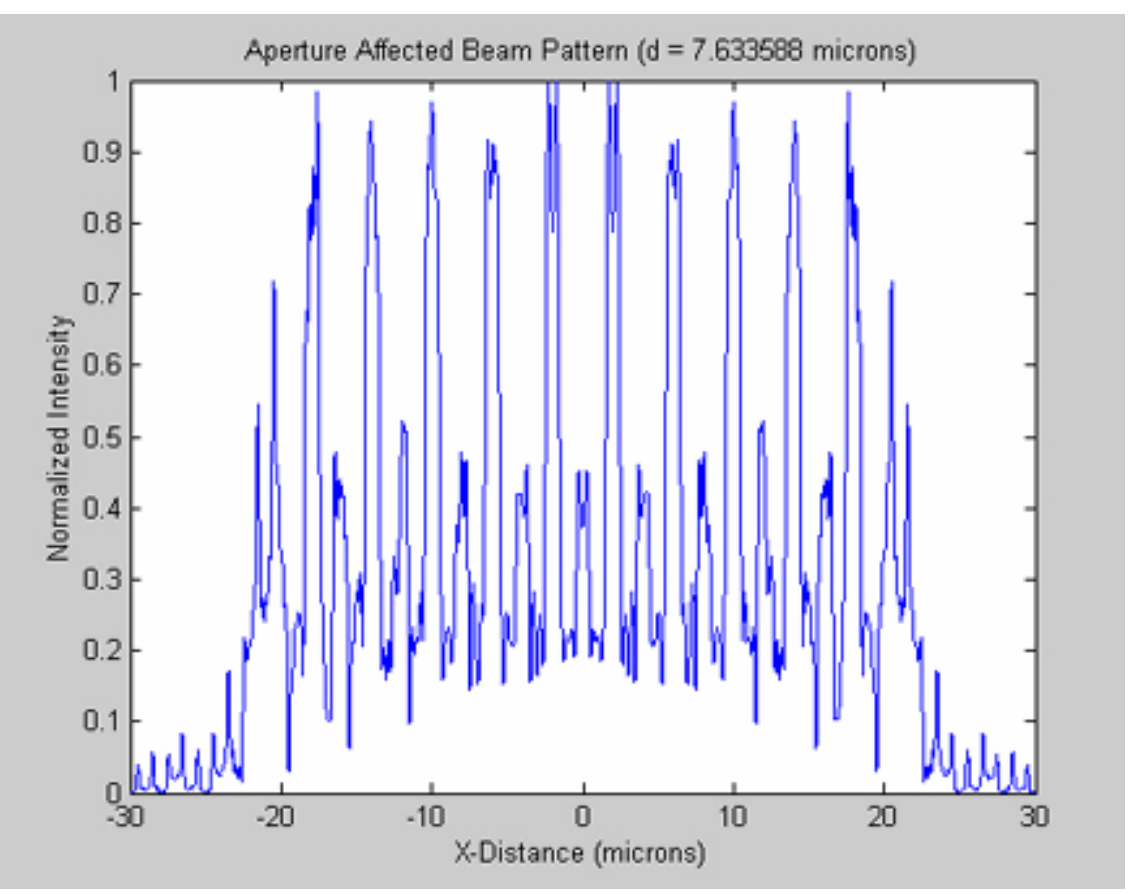

Figure 4.16: Grating effected output beam intensity profile, $\mathrm{N}_{\mathrm{F}}=10, \mathrm{~d}=7.63 \mu \mathrm{m}$.

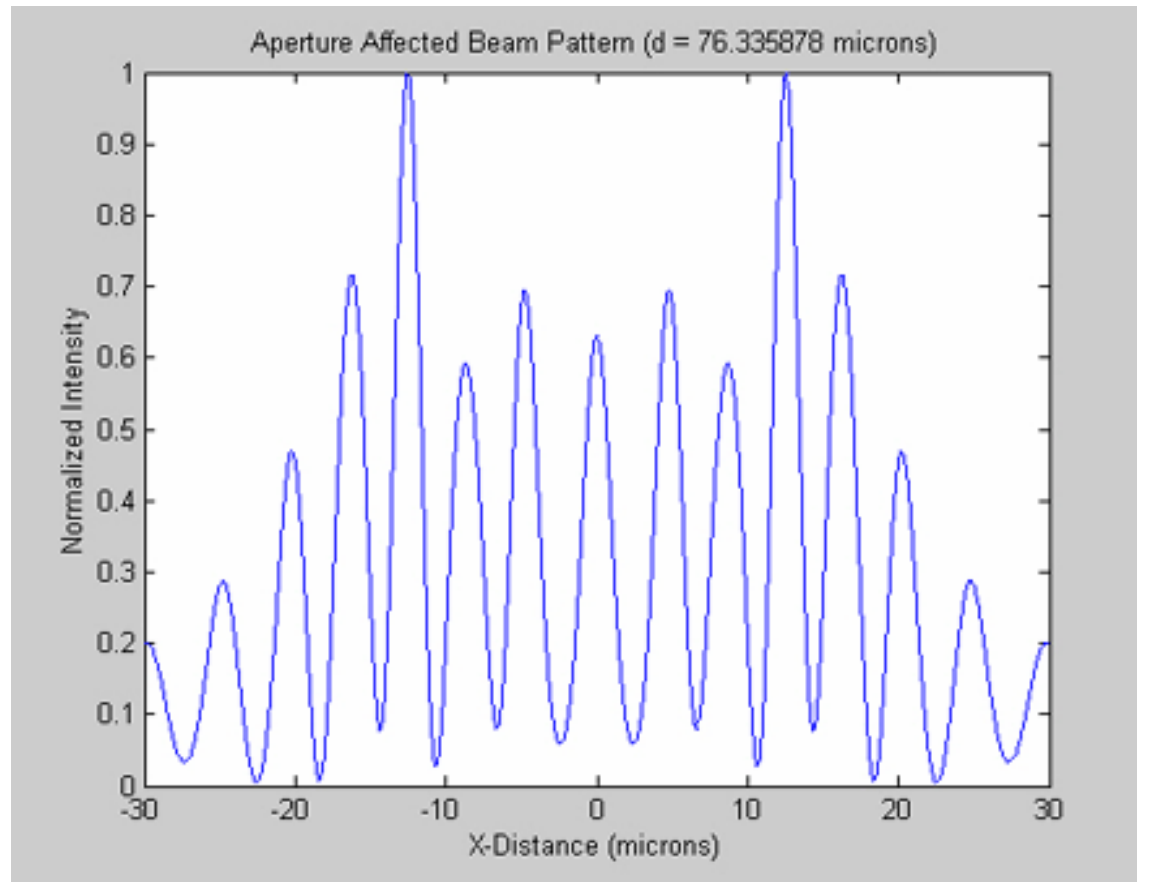

Figure 4.17: Grating effected output beam intensity profile, $N_{F}=1, d=76.33 \mu \mathrm{m}$. 


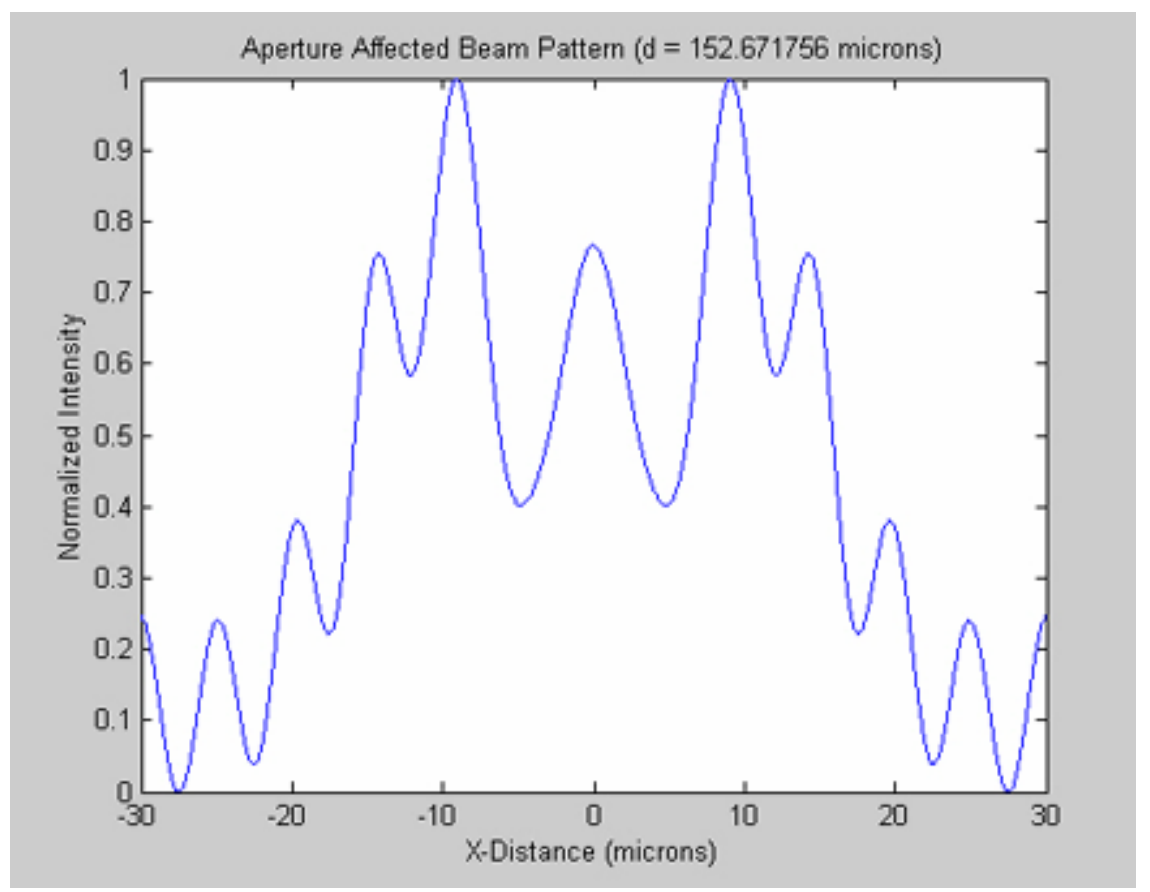

Figure 4.18: Grating effected output beam intensity profile, $\mathrm{N}_{\mathrm{F}}=0.5, \mathrm{~d}=153.67 \mu \mathrm{m}$.

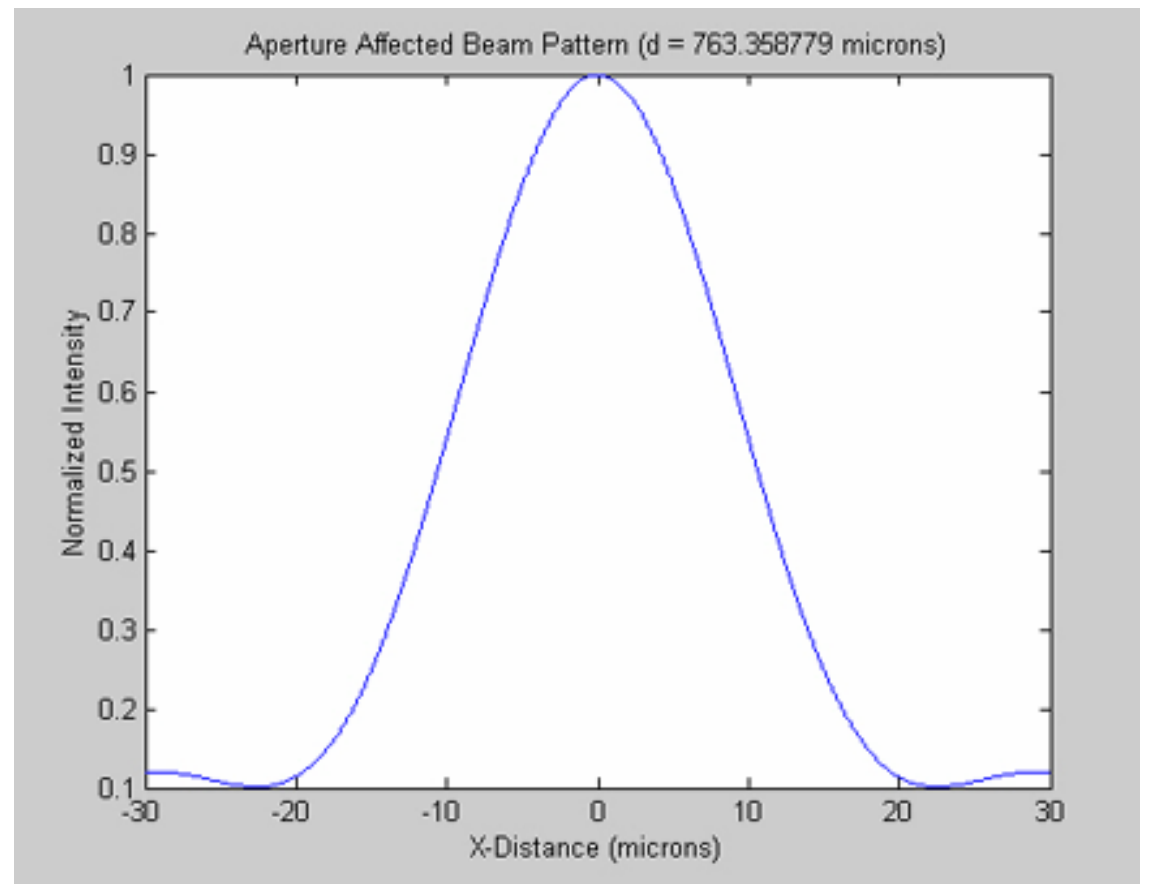

Figure 4.19: Grating effected output beam intensity profile, $N_{F}=0.1, d=763.36 \mu \mathrm{m}$. 


\subsection{Gaussian Beam Diffraction Through the Device Stage}

The next step in this examination is to determine the diffraction affects of the MEMS device stage on the through-wafer probe beam. In the current through-wafer probing setup, the output of a fiber is focused to a small spot and passed through the stage. This beam is Gaussian, having complex amplitude that can be expressed as described in Chapter 2 (Equations 2.2-7 - 2.2-12). The beam waist, $W_{0}$, or spot size in the plane of the device translation stage, was determined to be $\sim 10 \mu \mathrm{m}$ by inspection with an IR camera, and $z_{0}$ was calculated to be $\sim 20 \mu \mathrm{m}$ by measuring the spot size at the output of the lens system and the zaxis distance to W0. These values were used to compute the beam parameters included in $\mathrm{U}(\mathrm{r})$.

\subsubsection{Single Opening Diffraction Region}

The single-opening MEMS lateral comb resonator design (illustrated in Figure 4.20) was examined by treating the device stage as a transparent medium of varying thickness with a single rectangular opening (Appendix A, 'gausstrans.m'). The goal was to find the number of signal maxim incident on the detector as a result of 8 microns of stage motion $(4 \mu \mathrm{m}$ in both directions) in order to determine if enough information could be gathered from the output to accurately determine the position of the stage. These results are presented in Figure 4.21.

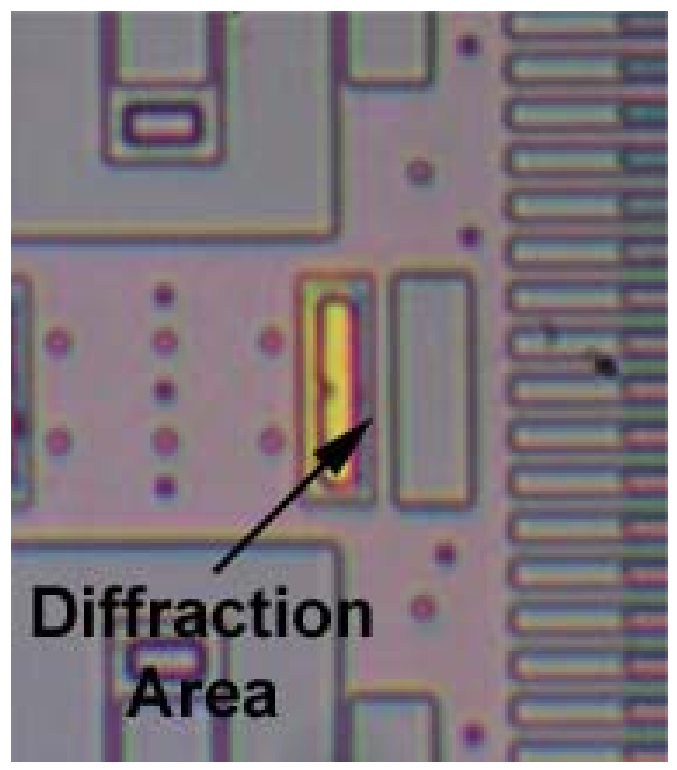

Figure 4.20: Single opening through-wafer probing area. 

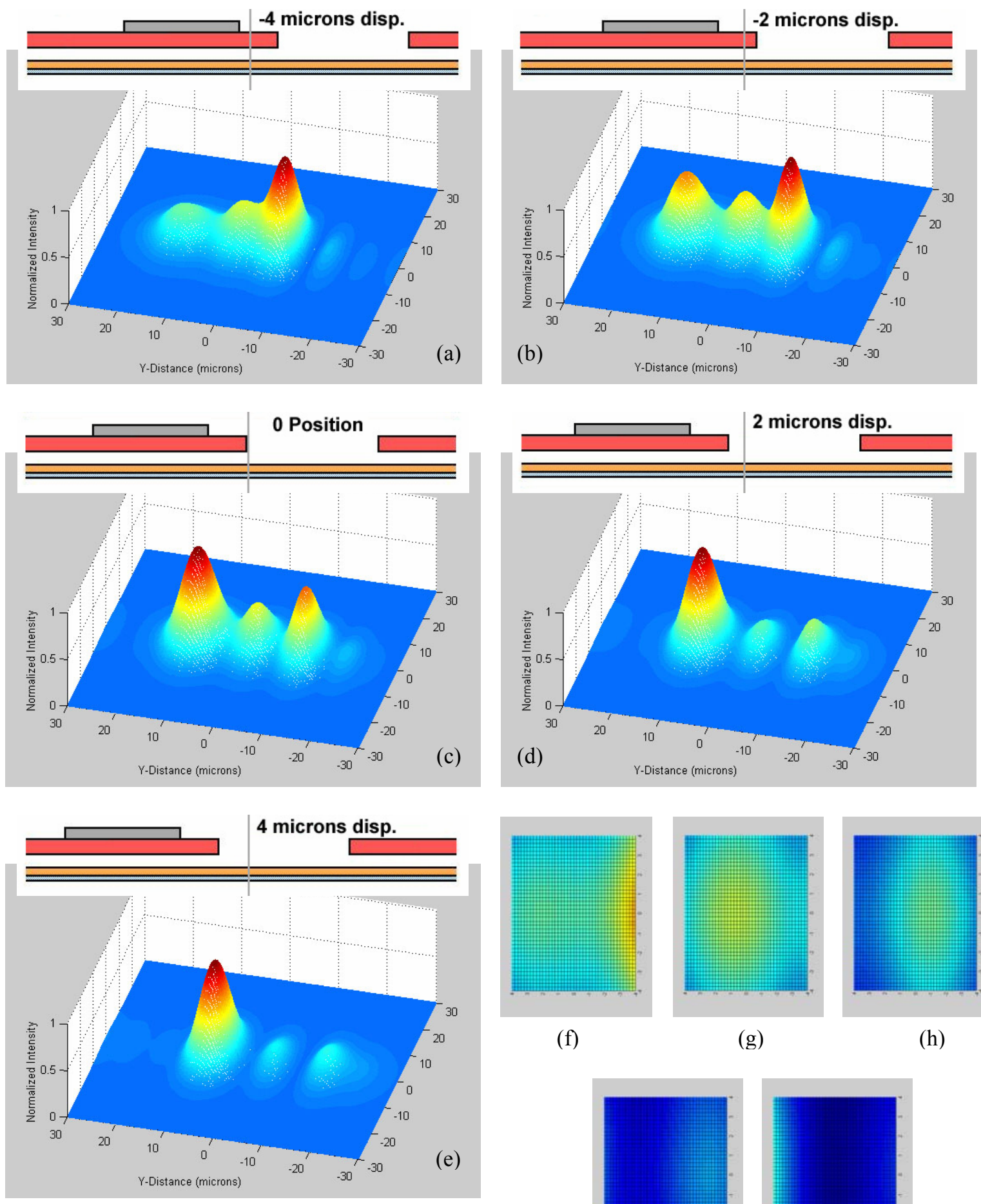

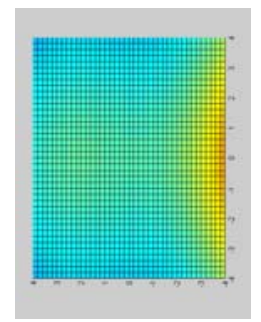

(f)

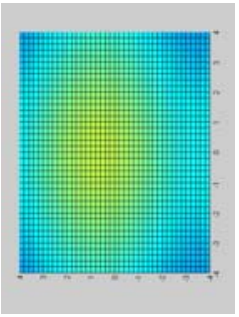

$(\mathrm{g})$

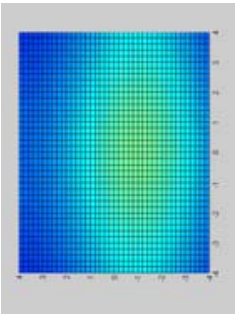

(h)

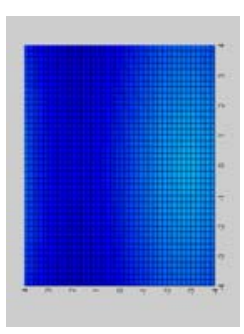

(i)

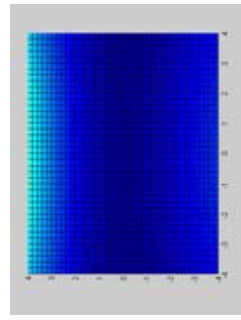

(j)

Figure 4.21: Through wafer diffraction pattern of a MEMS lateral comb resonator translation stage with a single single-opening for $8 \mu \mathrm{m}$ of travel and corresponding stage cross sections (to scale) (a)-(e). Detector intensity for the same range of motion (f)-(j). 
The optical field intensity at a distance of $100 \mu \mathrm{m}$ away from the device plane for each stage displacement was determined by theoretically passing a $20 \mu \mathrm{m}$ diameter spot through the aperture area. Cross sectional views illustrate the position of the stage opening and Poly 2 features for each displacement. It can be observed from these results that only one intensity maximum passes through the detector area over the entire range of motion exhibited.

\subsubsection{Closed-Loop Control Results}

Even though only on intensity change was evident over the entire range of device motion for single-opening stage geometries, the improved design of the optical microprobe setup, along with a real-time data acquisition board, allowed for simple linear proportional-integral (PI) and proportional-integral-differential (PID) control studies to be performed. Figure 4.22 shows the result of applying PI control to perform pulse impulse disturbance damping on a single-opening resonator device. 


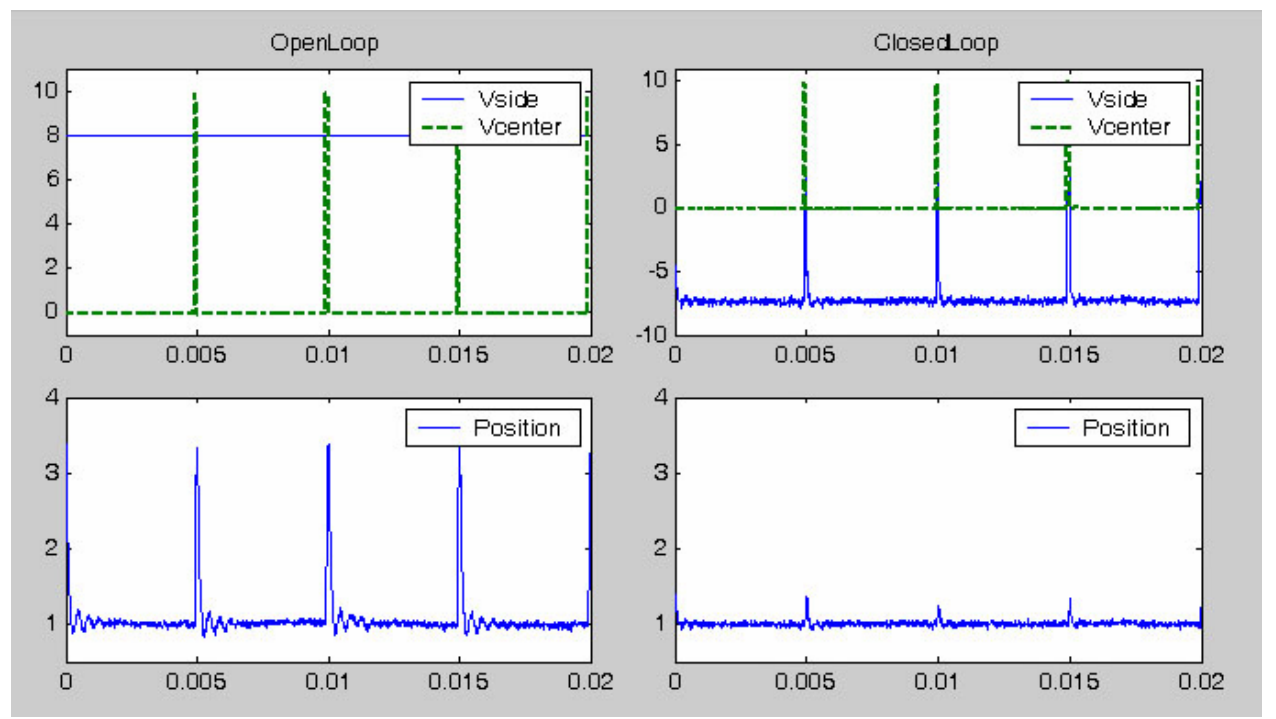

(a)

(b)
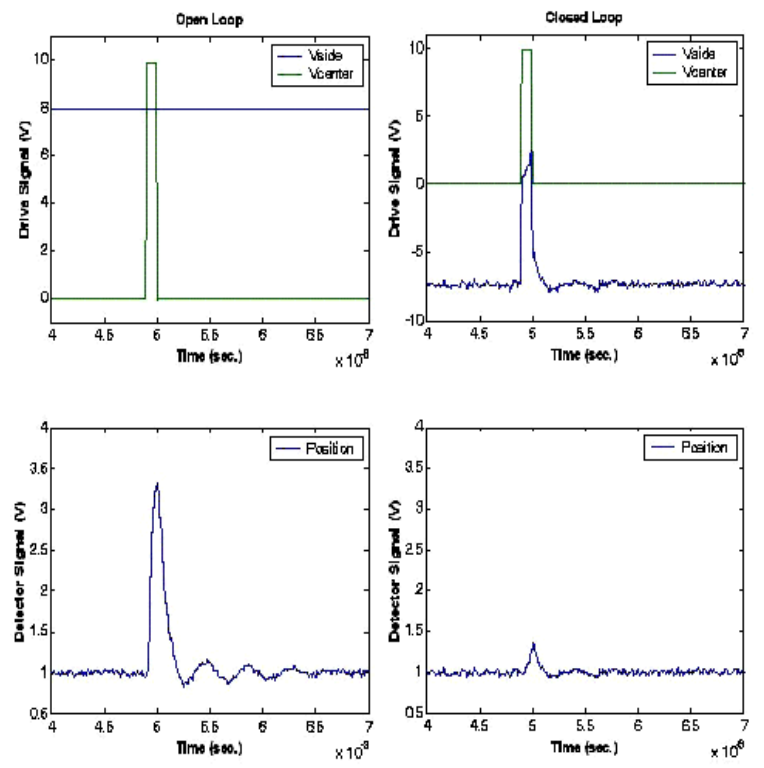

Figure 4.22: Active impulse disturbance damping (a) with close-up of single pulse response time (b). 
In steady state, the stator combs were held at positive and negative $8 \mathrm{~V}\left(\mathrm{~V}_{\text {SIDE }}\right)$ with the stage at rest at $0 \mathrm{~V}\left(\mathrm{~V}_{\text {CENTER }}\right)$. A $10 \mathrm{~V}$ impulse $100 \mu$ s in duration was then applied to the stage (top left, Figure 4.22(a) \& (b)) resulting in the received optical signal (in volts) shown in the bottom left plot of Figure 4.22(a) \& (b) when no control was applied, or the open-loop condition. Rapid displacement followed by mechanical ringing of the stage is clearly evident. The plots on the right hand side correspond to those on the left, but with the device under closed loop PI control, the effect of the voltage impulse on the position of the is nearly completely damped as a result of the adaptive response of the voltage on the stator combs with a response time of $\sim 1 \mu \mathrm{s}$.

Successful linear PID control has also been demonstrated under normal sinusoidal drive voltage operation of a lateral comb resonator using a peak detection algorithm. This result is shown in Figure 4.23.

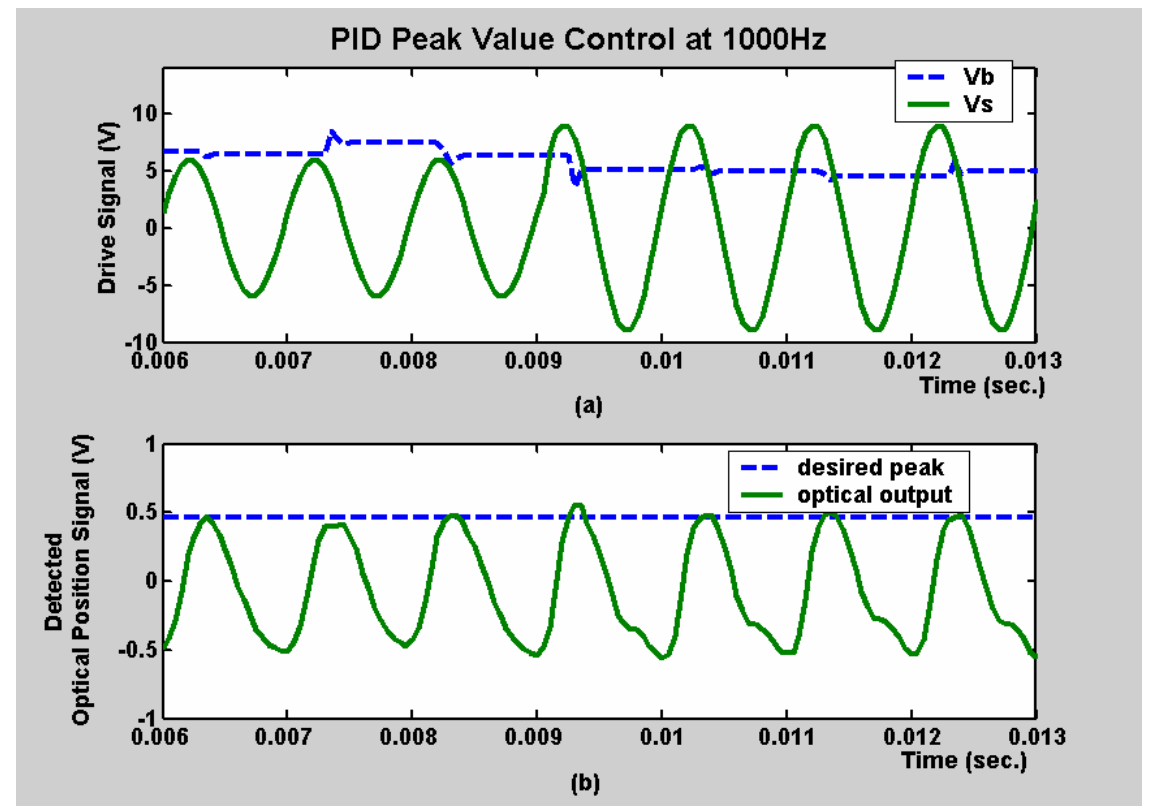

Figure 4.23: PID peak value control results.

The single-opening geometry device was driven with a $1 \mathrm{kHz} 12 \mathrm{~V}$ peak-to-peak on the stage of the device with stator voltages of positive and negative $7 \mathrm{~V}$. In the open-loop condition (not shown), a change in the magnitude of the sinusoidal drive voltage changes the amount of displacement exhibited by the stage, and, consequently, the dynamic range of the optical output voltage waveform. In the closed-loop condition illustrated in Figure 4.23, the stage 
was initially driven at the same voltage as the open-loop condition, and the sinusoidal voltage magnitude was increased (close to $9 \mathrm{~ms}$ in Figure 4.23). At this point, the peak detection algorithm changes the stator voltage to compensate for the increase in sinusoidal voltage and the consequent increase in displacement. This adjustment forces the peak optical output voltage level to return to the desired value (500 $\mathrm{mV}$ in this case) in a response time of $1 \mathrm{~ms}$.

Although the new optical microprobe setup provided a more stable platform with which to perform control experiments, the response times for dynamic MEMS closed-loop control remained slow compared to the resonance frequency of the devices tested $(2.5-3.5 \mathrm{kHz})$. More robust control methods could increase response times, but require a detailed system model and detailed positional information. Increased positional resolution can be achieved by examining grating structure geometries and the diffraction effects they have on the optical probe beam.

\subsubsection{Grating Diffraction Region}

A grating structure (Figure 4.24) was explored next using the same method, with the stage modeled as $2 \mu \mathrm{m}$ wide openings separated by $2 \mu \mathrm{m}$ wide polysilicon areas (Appendix A, 'gaussgrate.m'). Total grating area in the simulation is $30 \mu \mathrm{m}$ by $20 \mu \mathrm{m}$. Stage displacement was defined as a movement of 4 microns in one direction from the at-rest position. Results are presented in Figure 4.25.

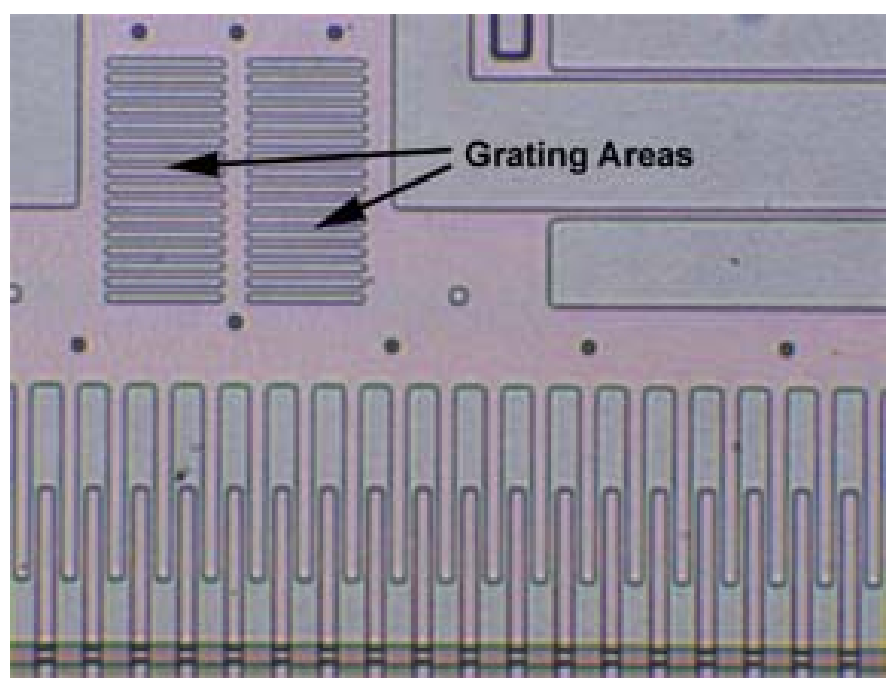

Figure 4.24: Grating structure through-wafer probing area. 

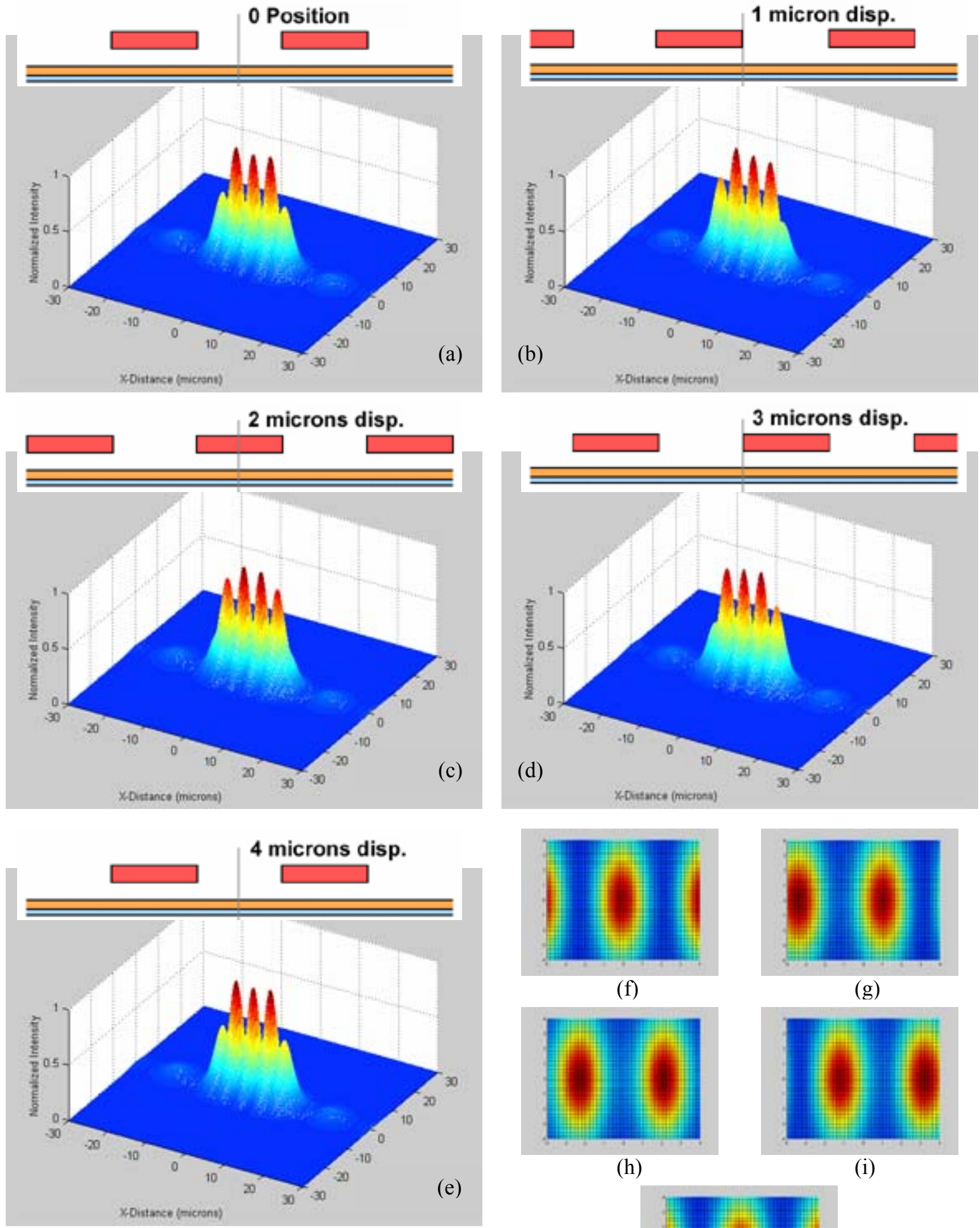

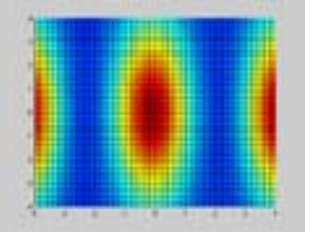

(f)

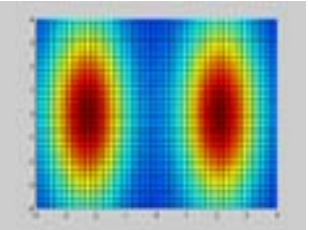

(h)

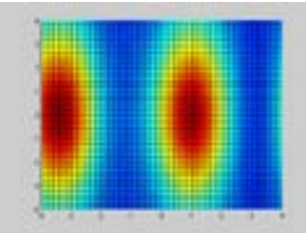

$(\mathrm{g})$

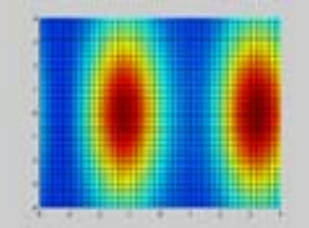

(i)

Figure 4.25: Through wafer diffraction pattern of a MEMS lateral comb resonator translation stage with a diffraction grating for $4 \mu \mathrm{m}$ of travel and corresponding stage cross sections (not to scale) (a)(e). Detector intensity for the same range of motion

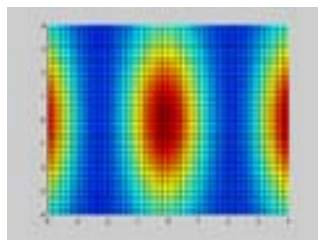

(j) 
In this case, a spot size of $10 \mu \mathrm{m}$ was used and the optical field intensity was observed $25 \mu \mathrm{m}$ away from the device plane. It can be seen in Figure 4.25 (f)-(j) that stage travel from the zero position to $4 \mu \mathrm{m}$ displacement causes two intensity maxim to pass through the area of the detector, giving a resolution of 4 microns per intensity maximum.

\subsubsection{Theoretical and Experimental Grating Signal Examination}

After the positional resolution of the moving grating structure was determined, a study to determine the validity of the optical signal pattern of the moving device stage was undertaken. The program used to calculate the optical field intensity was modified to accept a vector of displacement values obtained from a Matlab SimuLink system model representation of the device, shown in Figure 4.26 (Appendix A, 'outputsim1d.m').

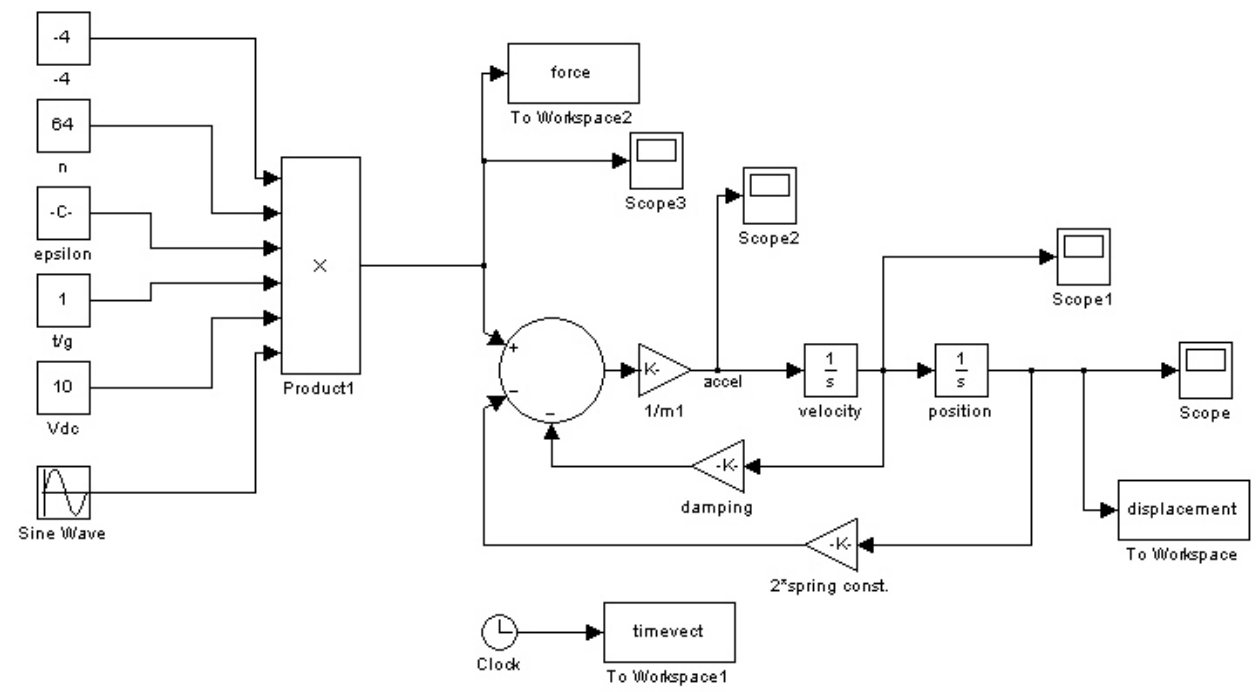

Figure 4.26: Lateral comb resonator system model.

This model was developed using Equation 2.1-1, with mass, spring constant, and damping values determined by the frequency sweep and curve fitting method described in Chapter $2\left(m=3.2 \cdot 10^{-10} \mathrm{~kg}, k=0.05346\right.$, and $\left.\beta=1.15 \cdot 10^{-6}\right)$. The output from the simulation program gives a time varying cross-section of the intensity incident on the area of the $8 \mu \mathrm{m}$ diameter detector fiber as the grating of the device stage moves sinusiodally. Simulation results for various frequencies between 1000 and $5000 \mathrm{~Hz}$ were performed and compared to 
experimental optical output data at the same frequencies. Results are presented in Figures 4.27-4.45.

To assure that peak intensity would occur at the zero position of the stage in experimental data, die adjustment was performed with no voltage applied to the device to give the maximum intensity incident on the detector fiber when the probe beam was located under the grating area. This zero position is identical to the stage position indicated in Figure 4.25(a). 
(a)

Theoretical Optical Output and Drive Force at $1000 \mathrm{~Hz}$

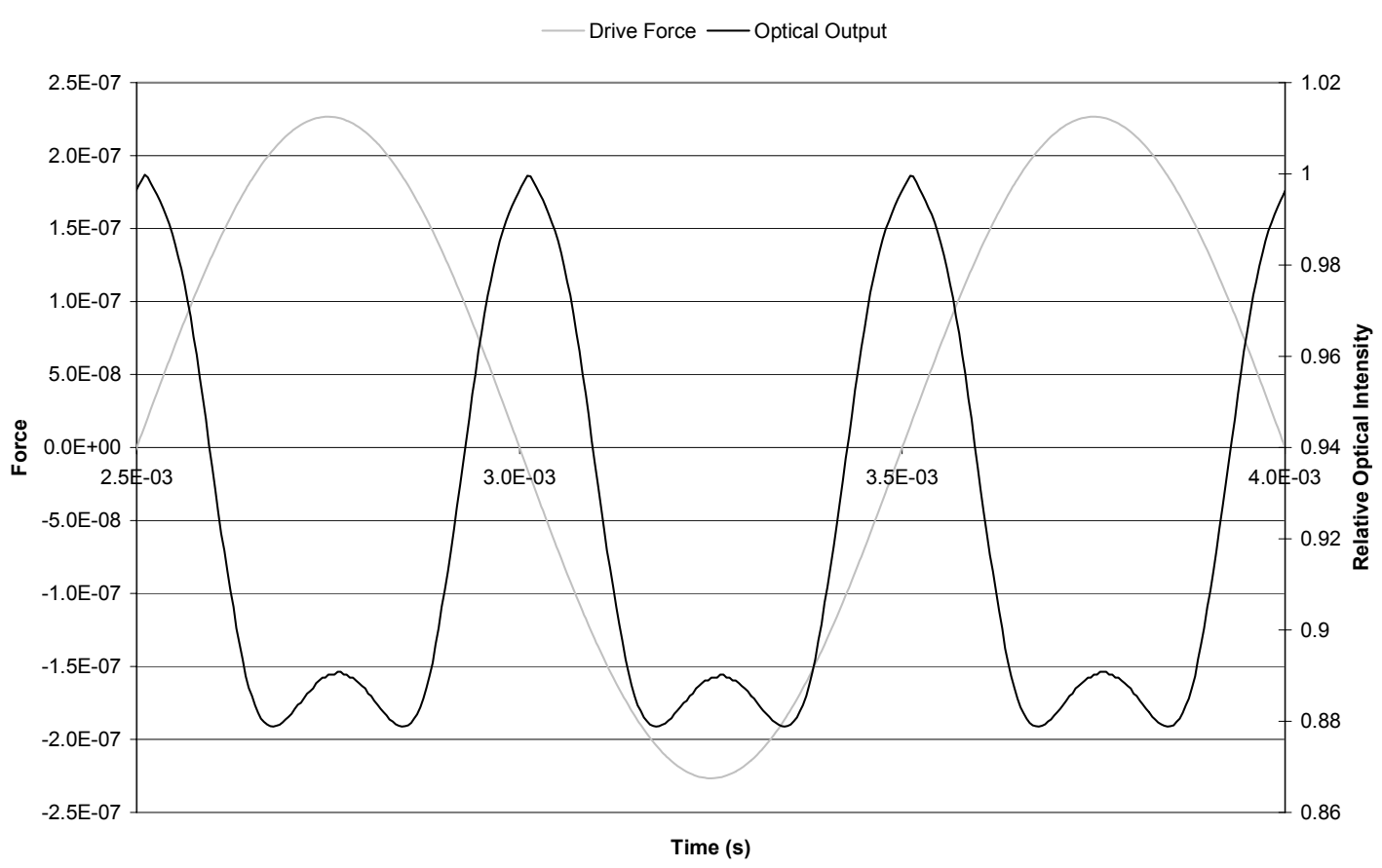

(b)

Experimental Optical Output and Drive Voltage at $1000 \mathrm{~Hz}$

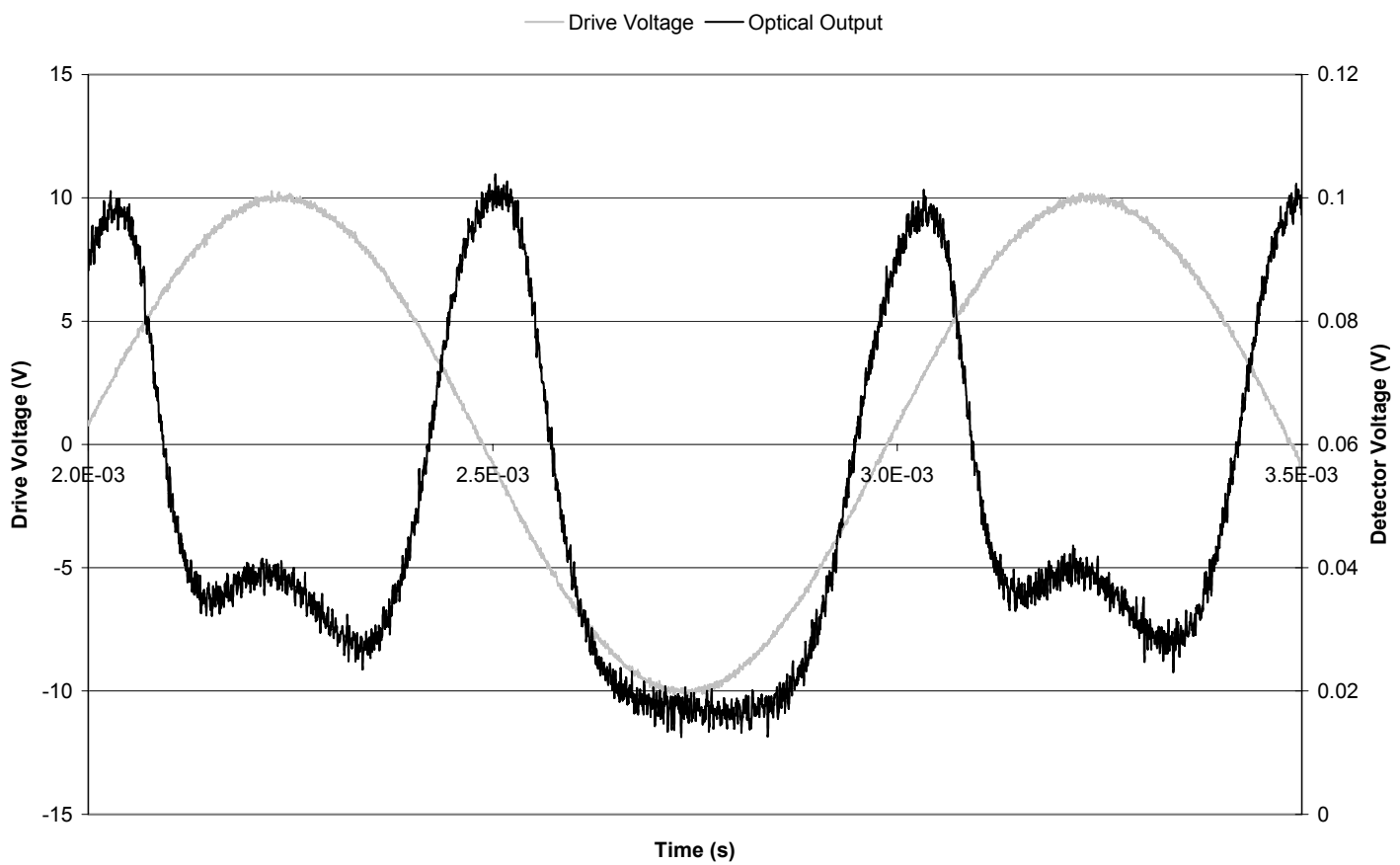

Figure 4.27: Optical output comparison at $1000 \mathrm{~Hz}$ : (a) theoretical results and (b) experimental data. 
(a)

Theoretical Optical Output and Drive Force at $1500 \mathrm{~Hz}$

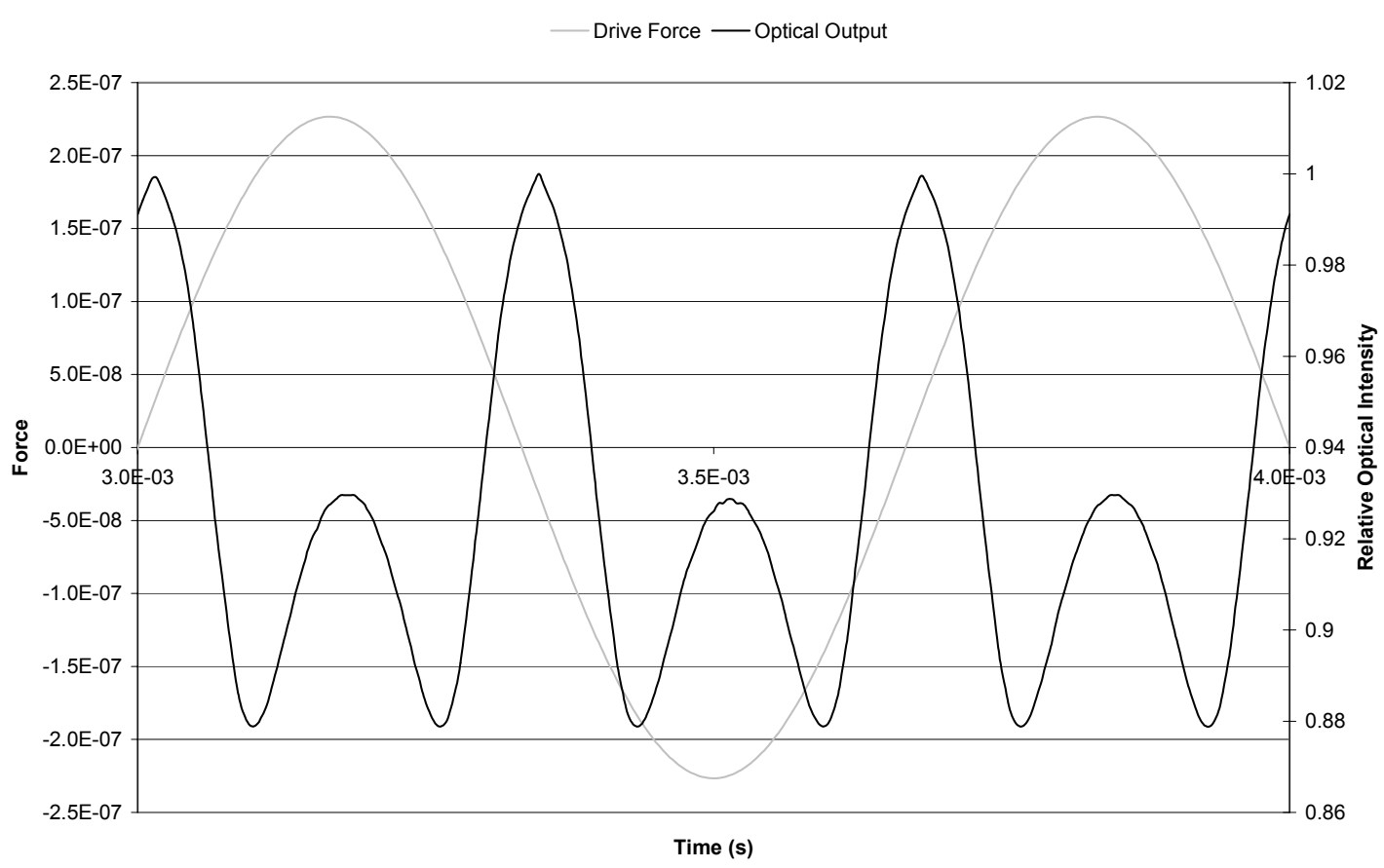

(b)

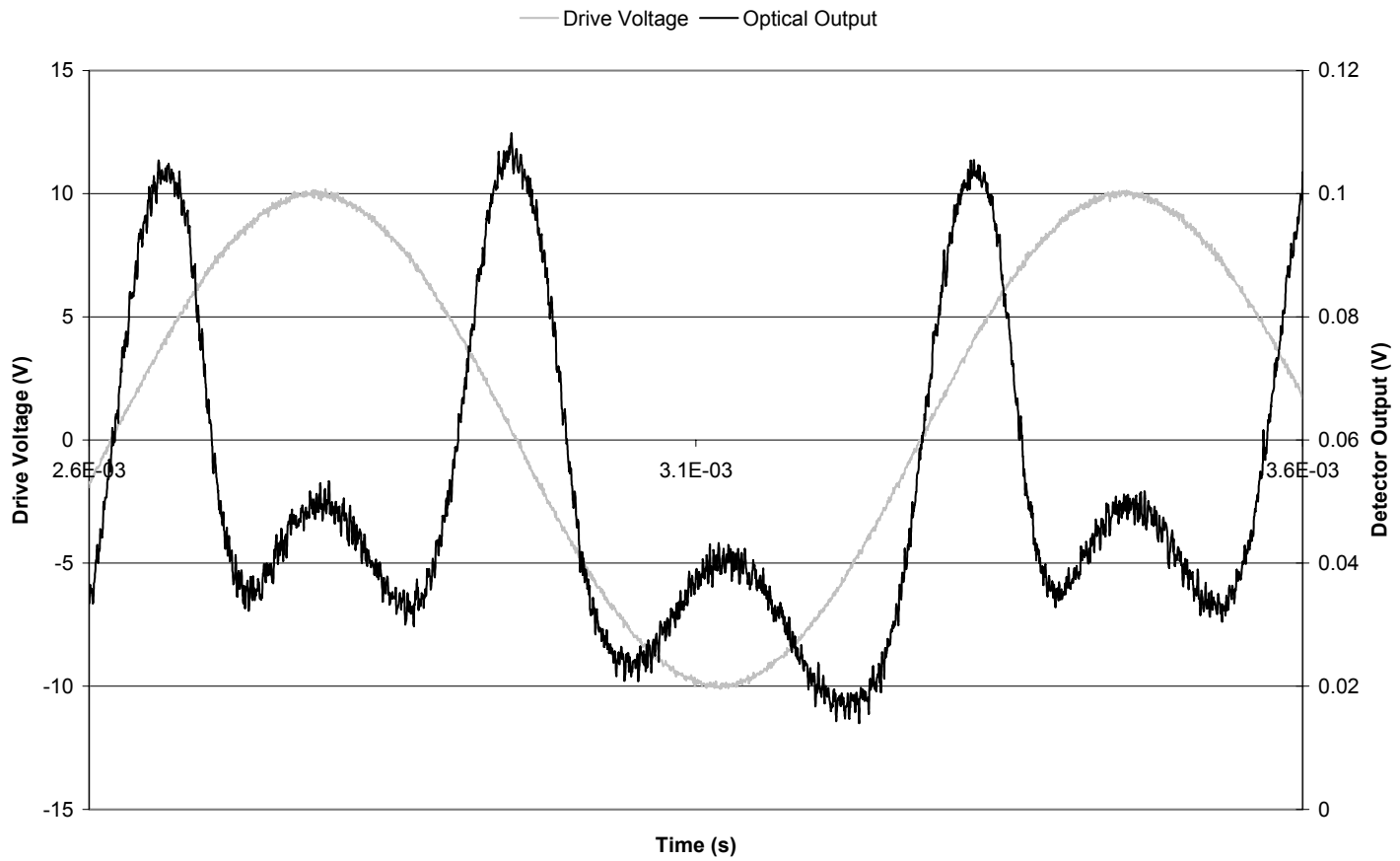

Figure 4.28: Optical output comparison at $1500 \mathrm{~Hz}$ : (a) theoretical results and (b) experimental data. 
(a)

Theoretical Optical Output and Drive Force at $2000 \mathrm{~Hz}$

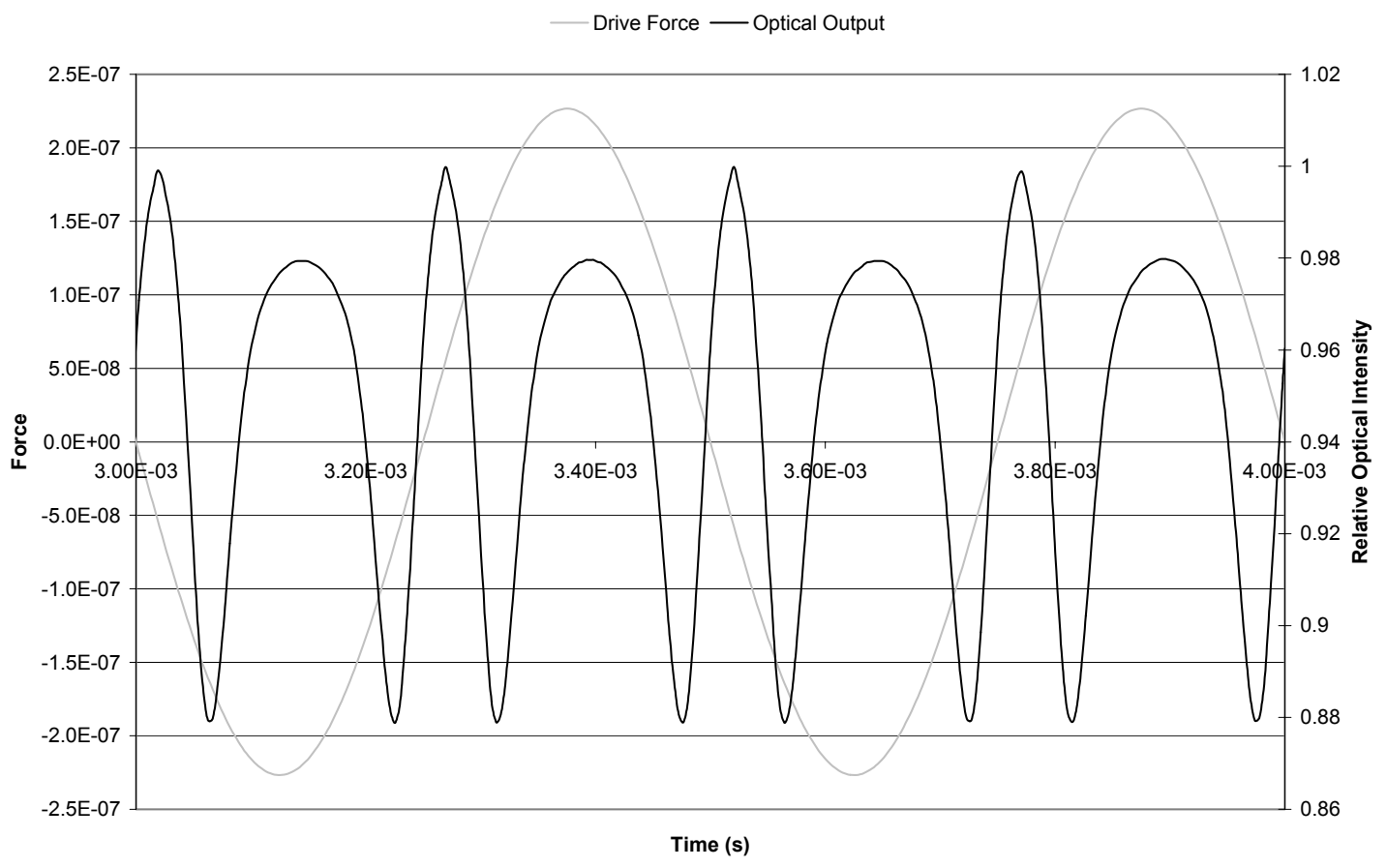

(b)

Experimental Optical Output and Drive Voltage at $2000 \mathrm{~Hz}$

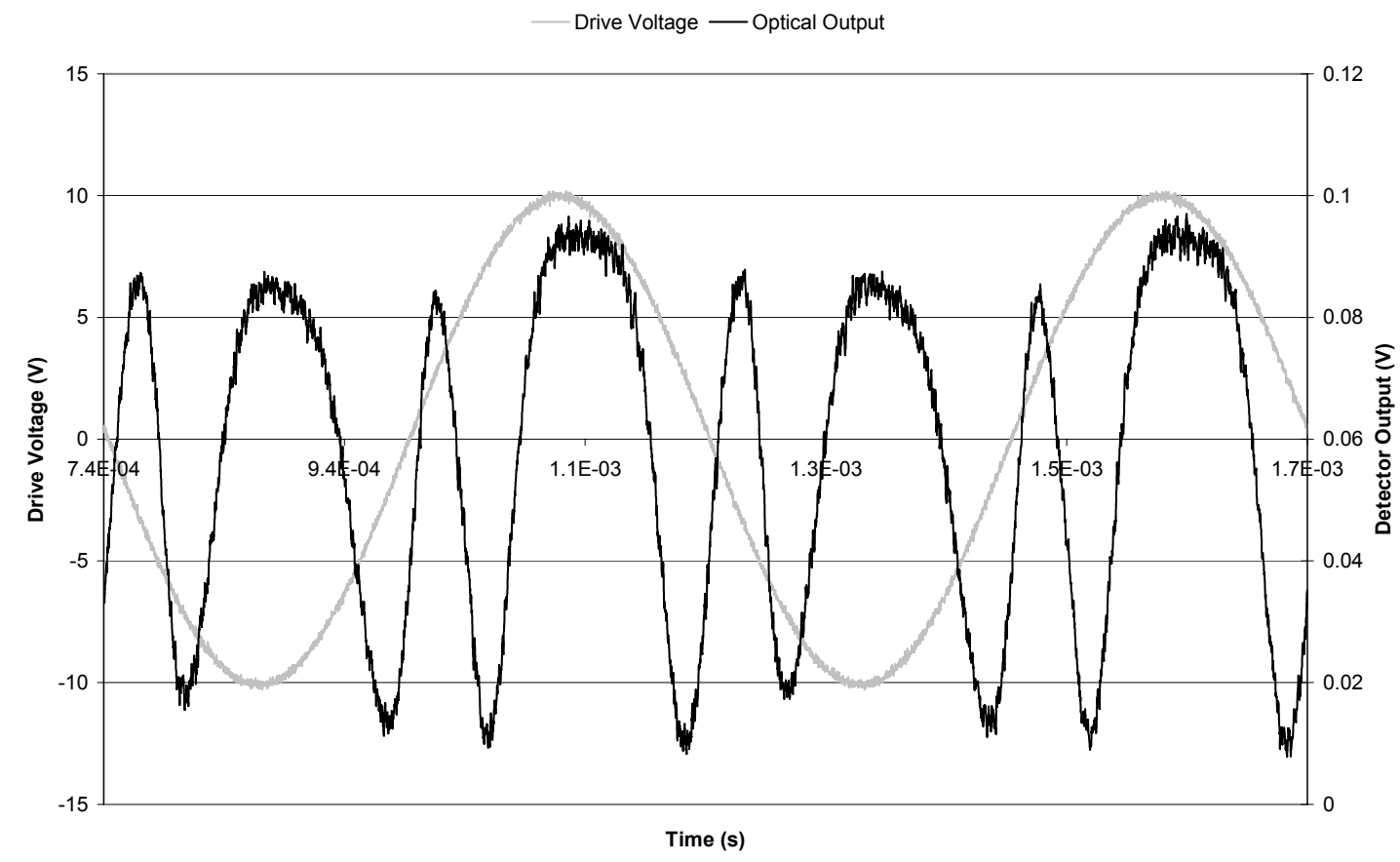

Figure 4.29: Optical output comparison at $2000 \mathrm{~Hz}$ : (a) theoretical results and (b) experimental data. 
(a)

Theoretical Optical Output and Drive Force at $2200 \mathrm{~Hz}$

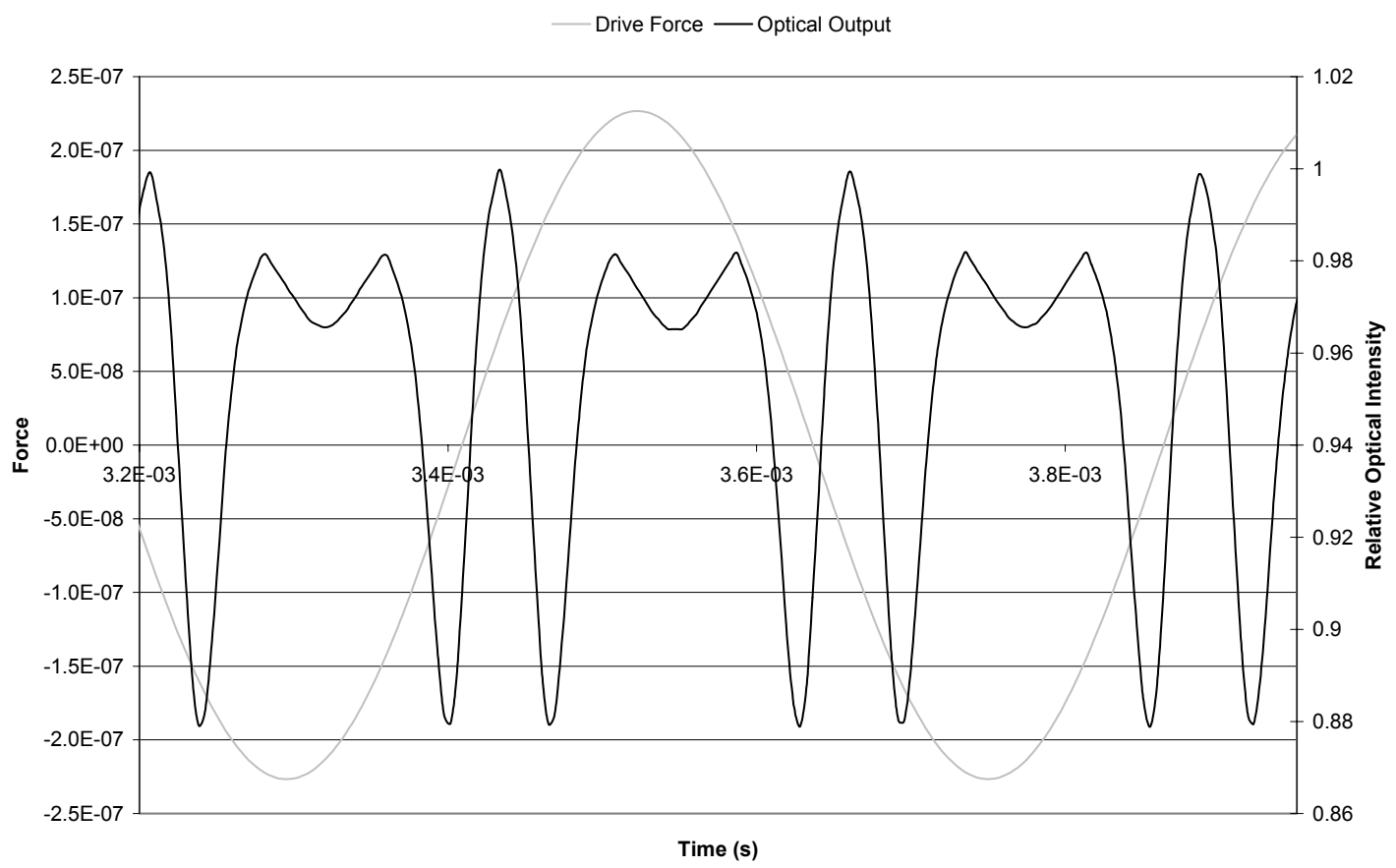

(b)

Experimental Optical Output and Drive Voltage at $2200 \mathrm{~Hz}$

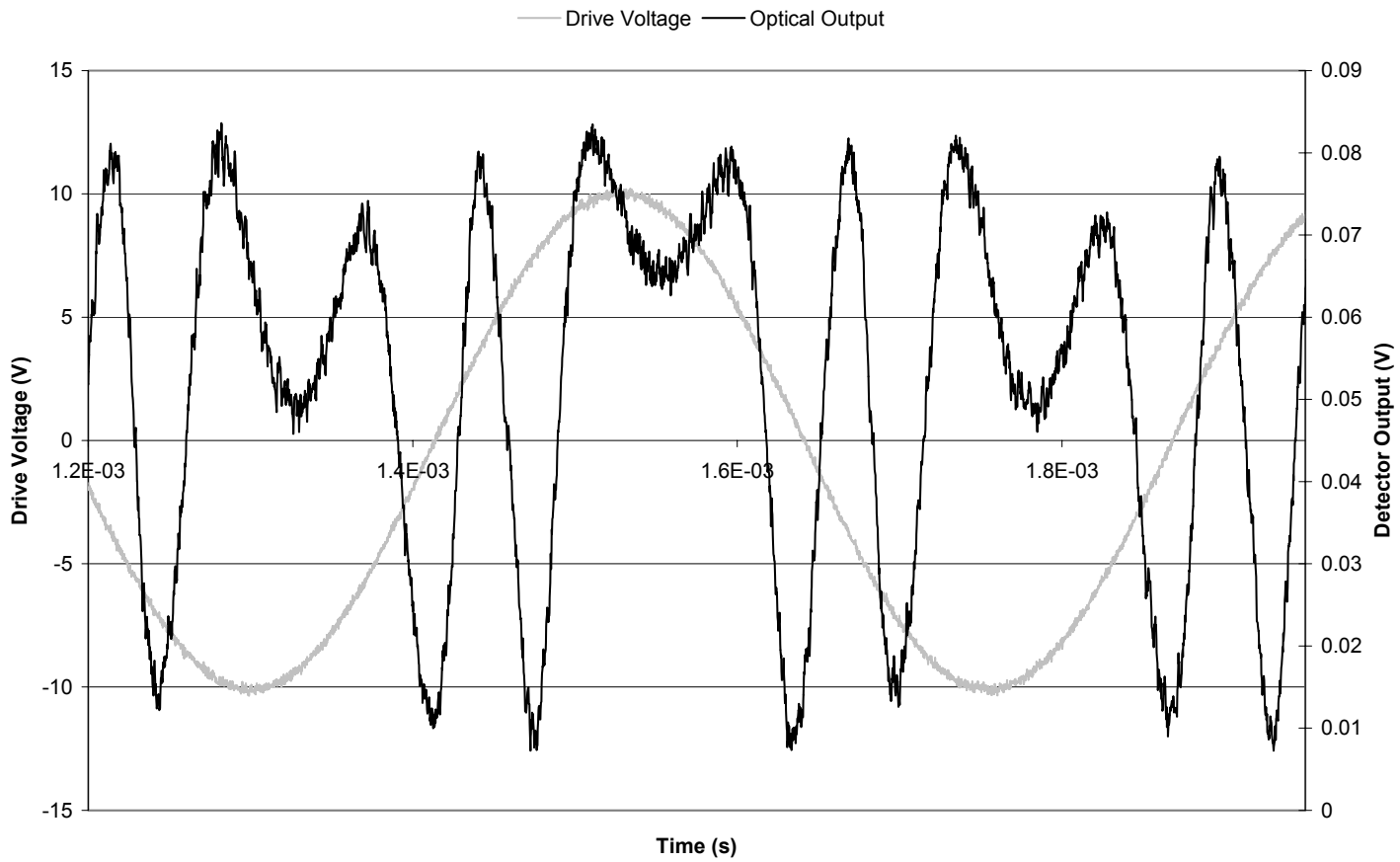

Figure 4.30: Optical output comparison at $2200 \mathrm{~Hz}$ : (a) theoretical results and (b) experimental data. 
(a)

Theoretical Optical Output and Drive Force at $2400 \mathrm{~Hz}$

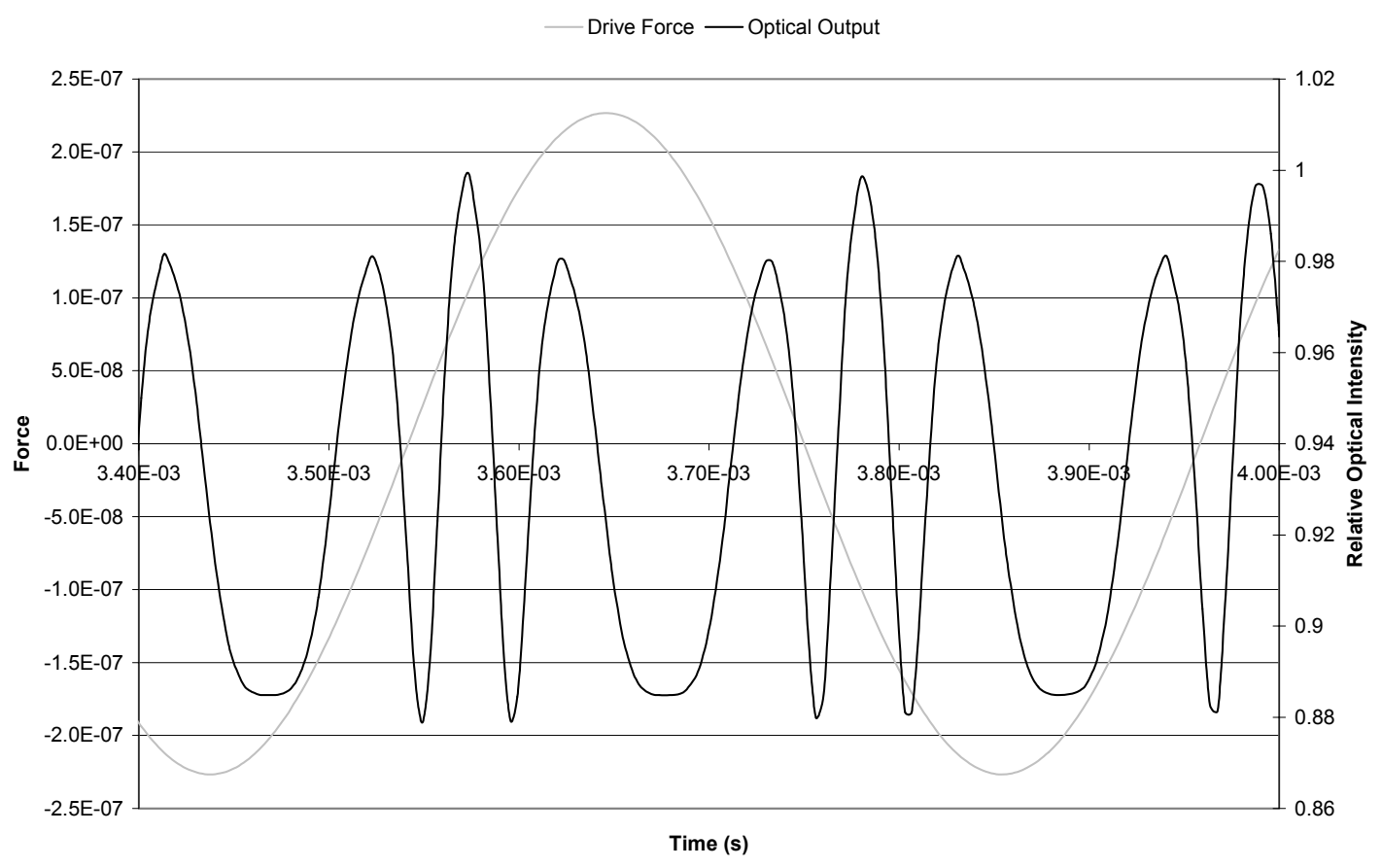

(b)

Experimental Optical Output and Drive Voltage at $2400 \mathrm{~Hz}$

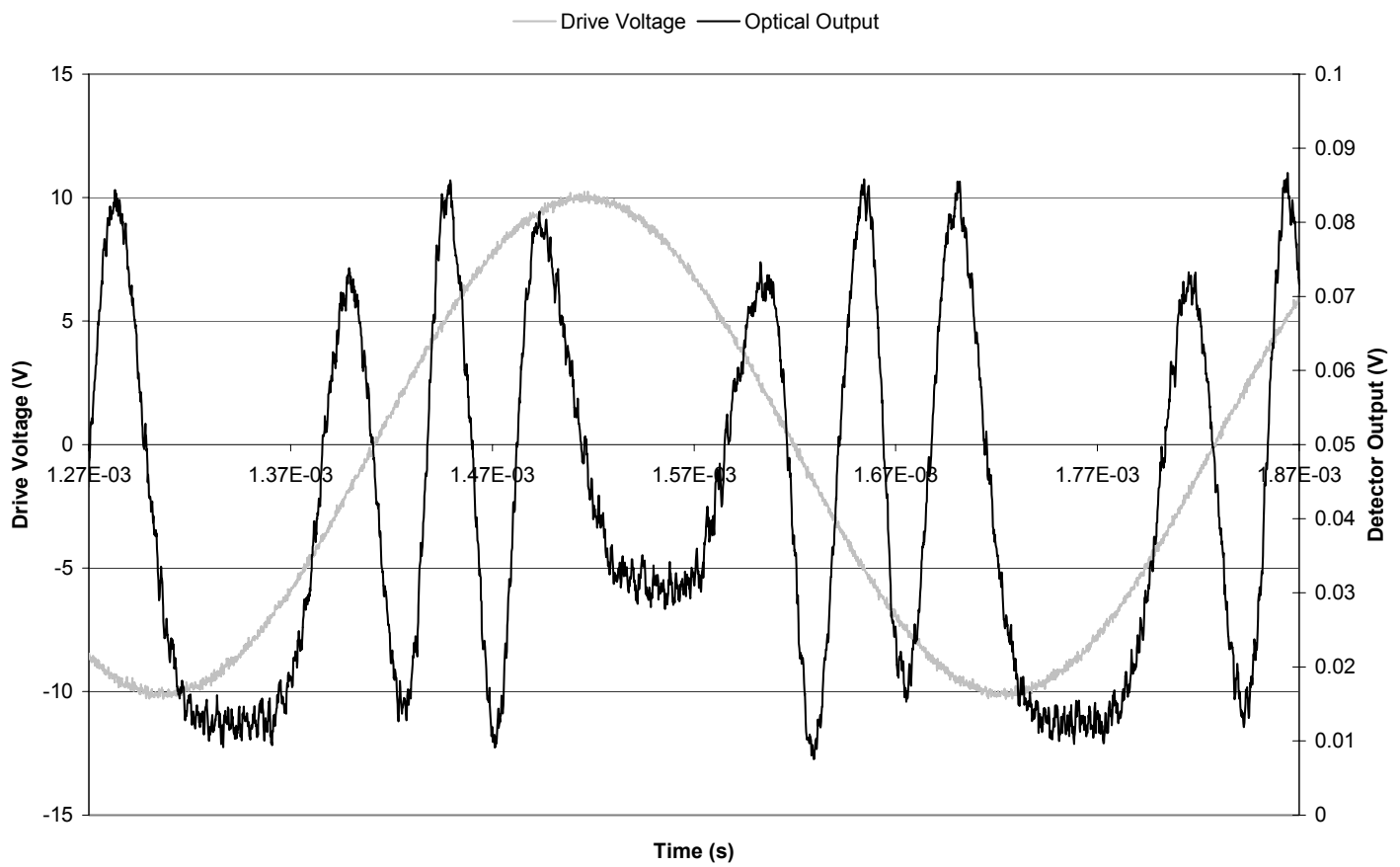

Figure 4.31: Optical output comparison at $2400 \mathrm{~Hz}$ : (a) theoretical results and (b) experimental data. 
(a)

Theoretical Optical Output and Drive Force at $2600 \mathrm{~Hz}$

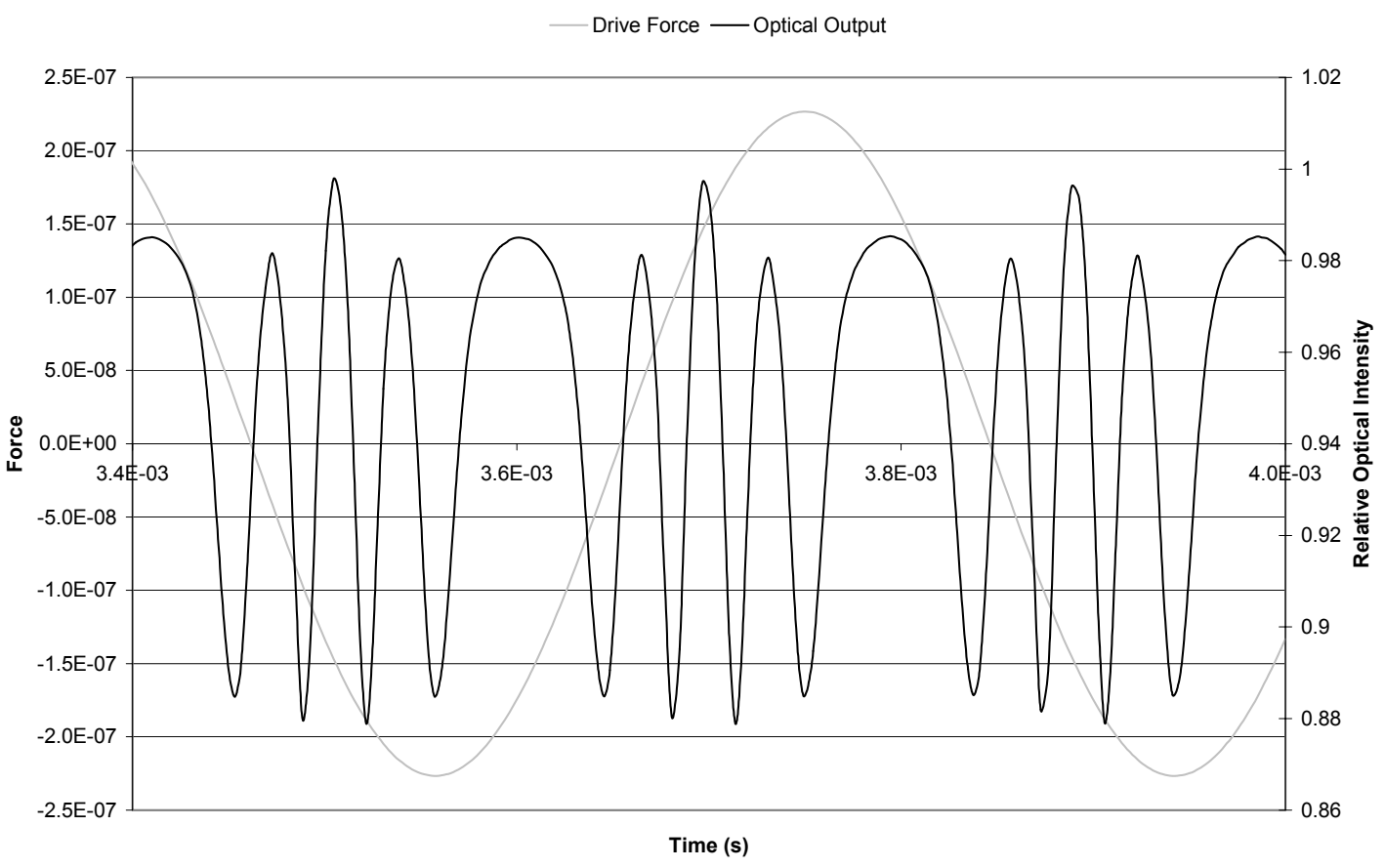

(b)

Experimental Optical Ouput and Drive Voltage at $2600 \mathrm{~Hz}$

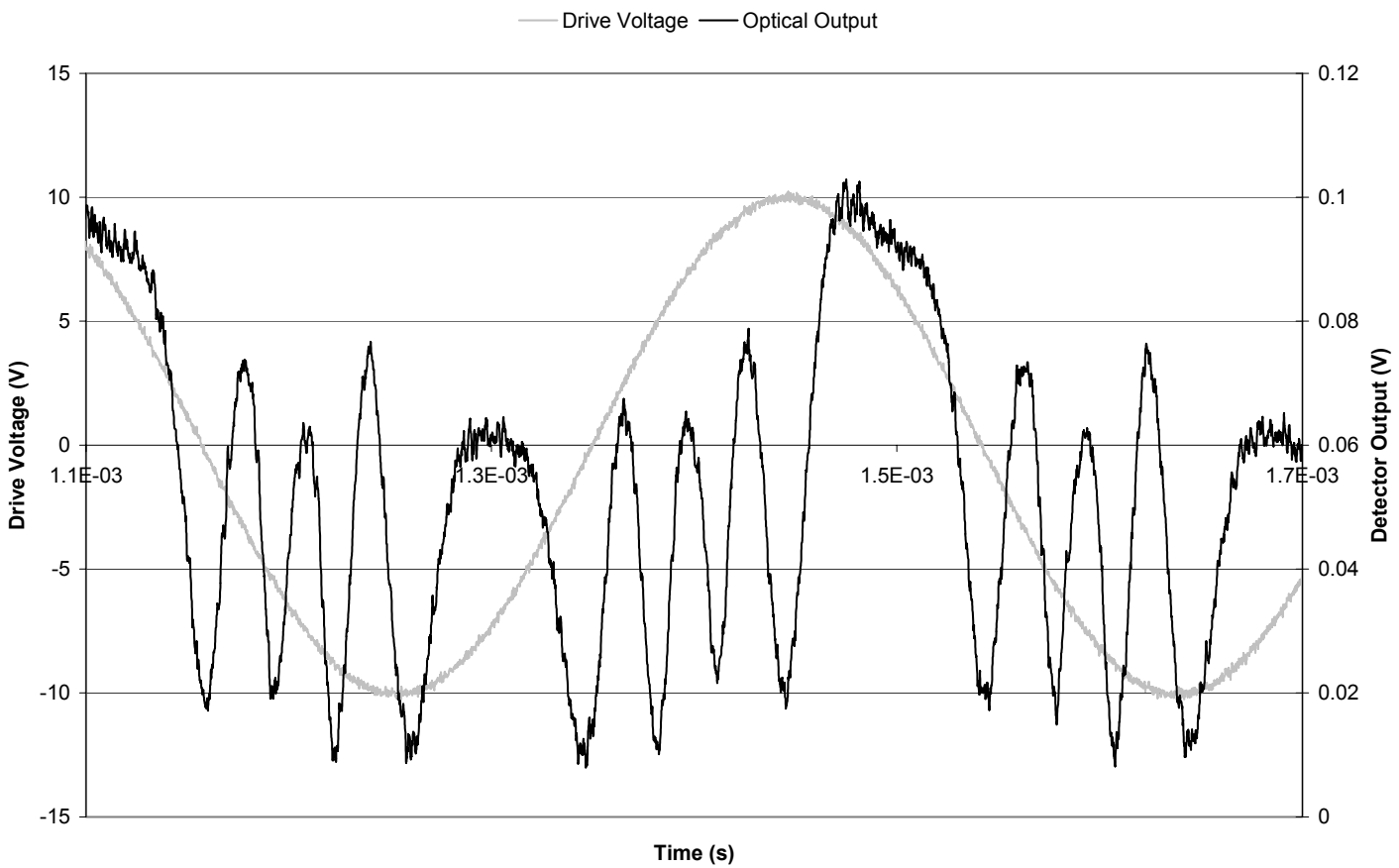

Figure 4.32: Optical output comparison at $2600 \mathrm{~Hz}$ : (a) theoretical results and (b) experimental data. 
(a)

Theoretical Optical Output and Drive Force at $2700 \mathrm{~Hz}$

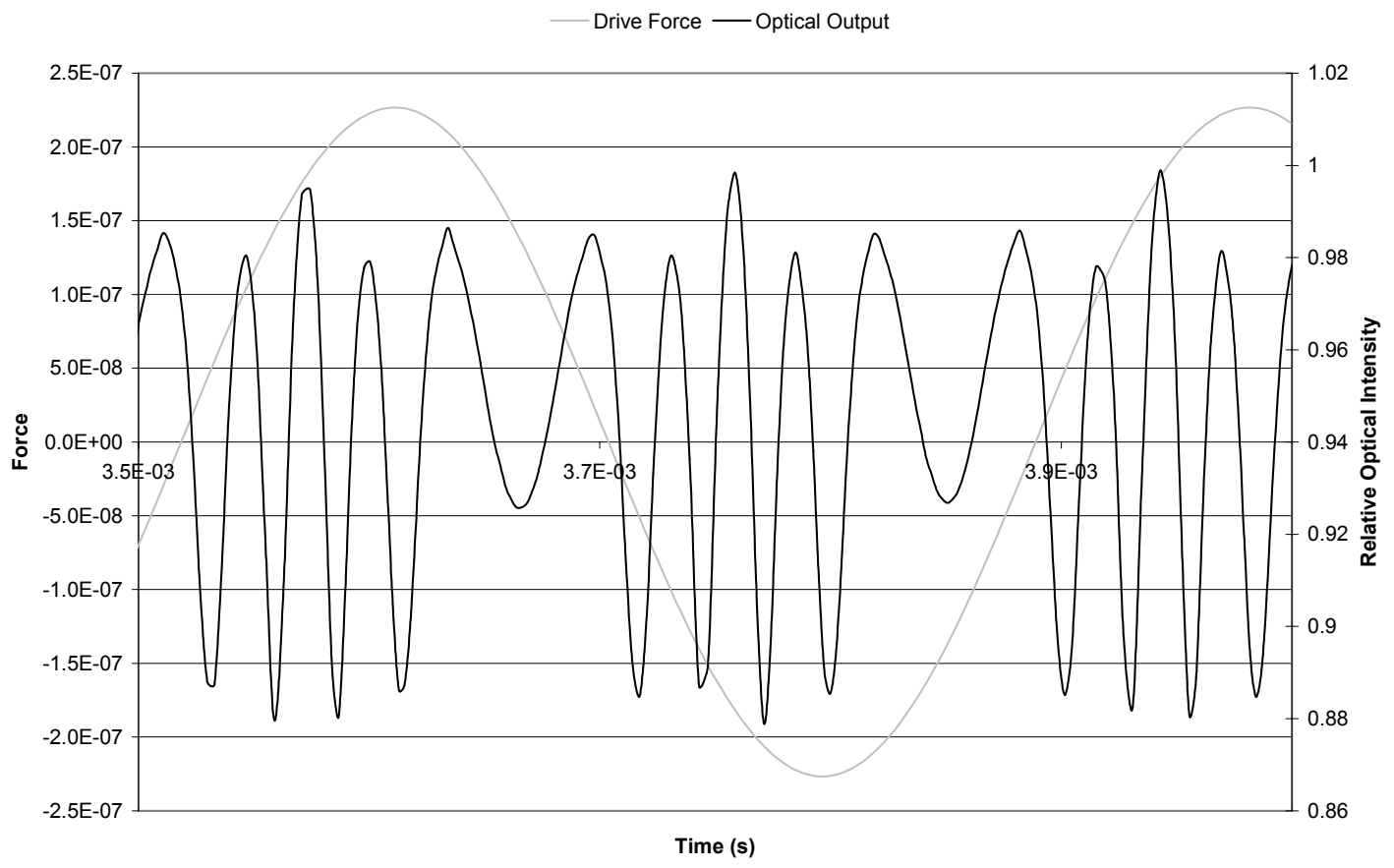

(b)

Experimental Optical Output and Drive Voltage at $2700 \mathrm{~Hz}$

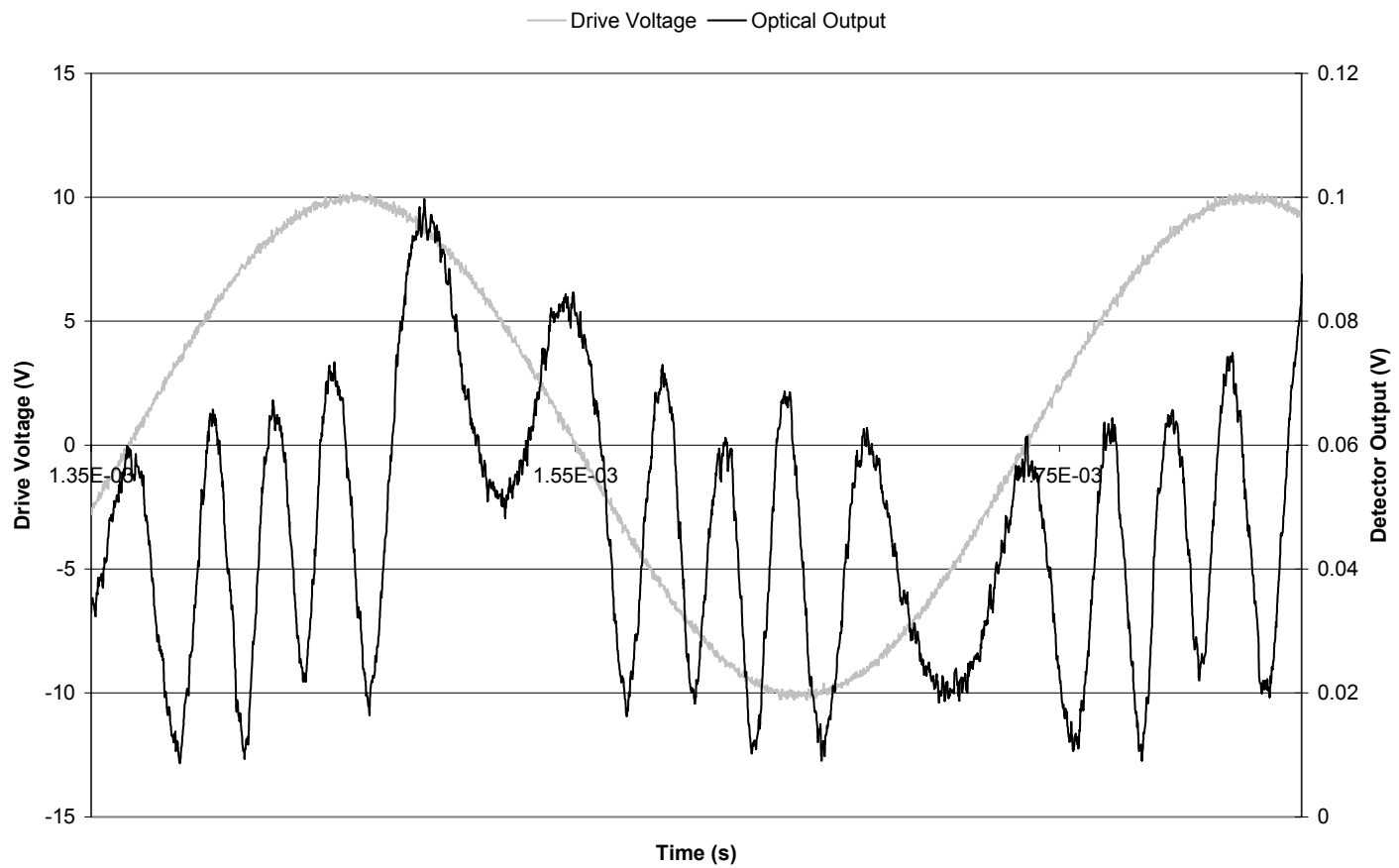

Figure 4.33: Optical output comparison at $2700 \mathrm{~Hz}$ : (a) theoretical results and (b) experimental data. 
(a)

Theoretical Optical Output and Drive Force at $2800 \mathrm{~Hz}$

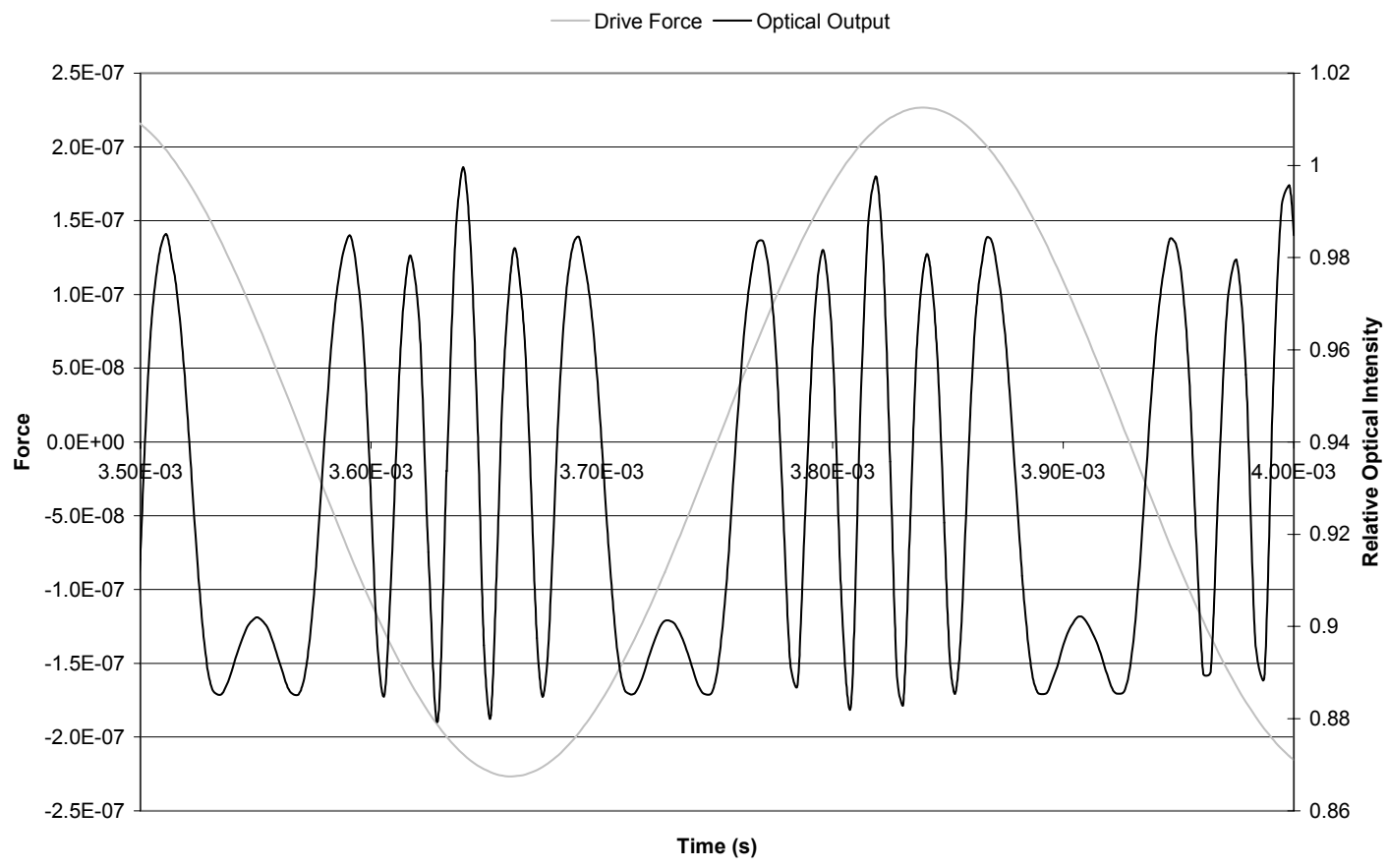

(b)

Experimental Optical Output and Drive Force at $2800 \mathrm{~Hz}$

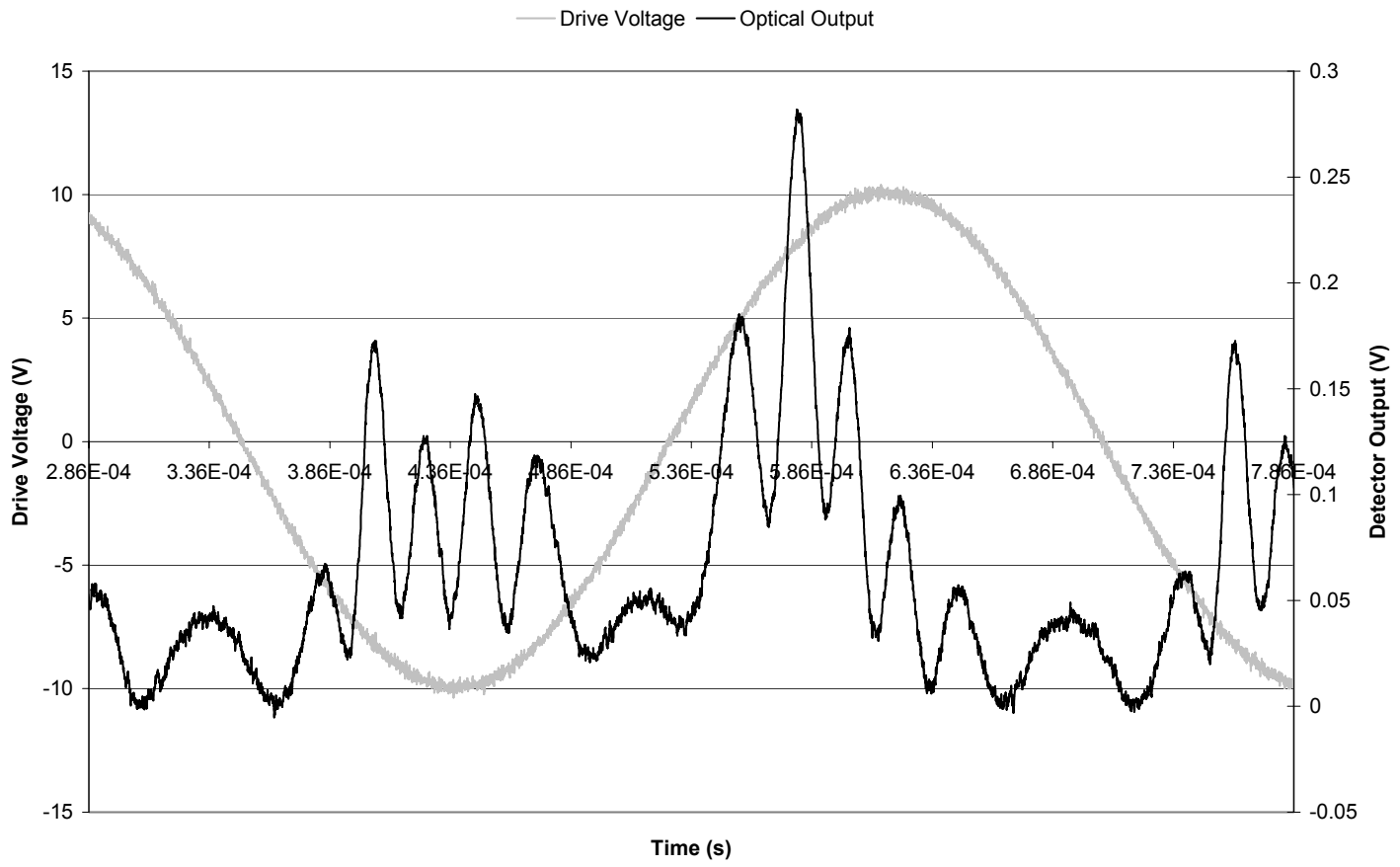

Figure 4.34: Optical output comparison at $2800 \mathrm{~Hz}$ : (a) theoretical results and (b) experimental data. 
(a)

Theoretical Optical Output and Drive Force at $2900 \mathrm{~Hz}$

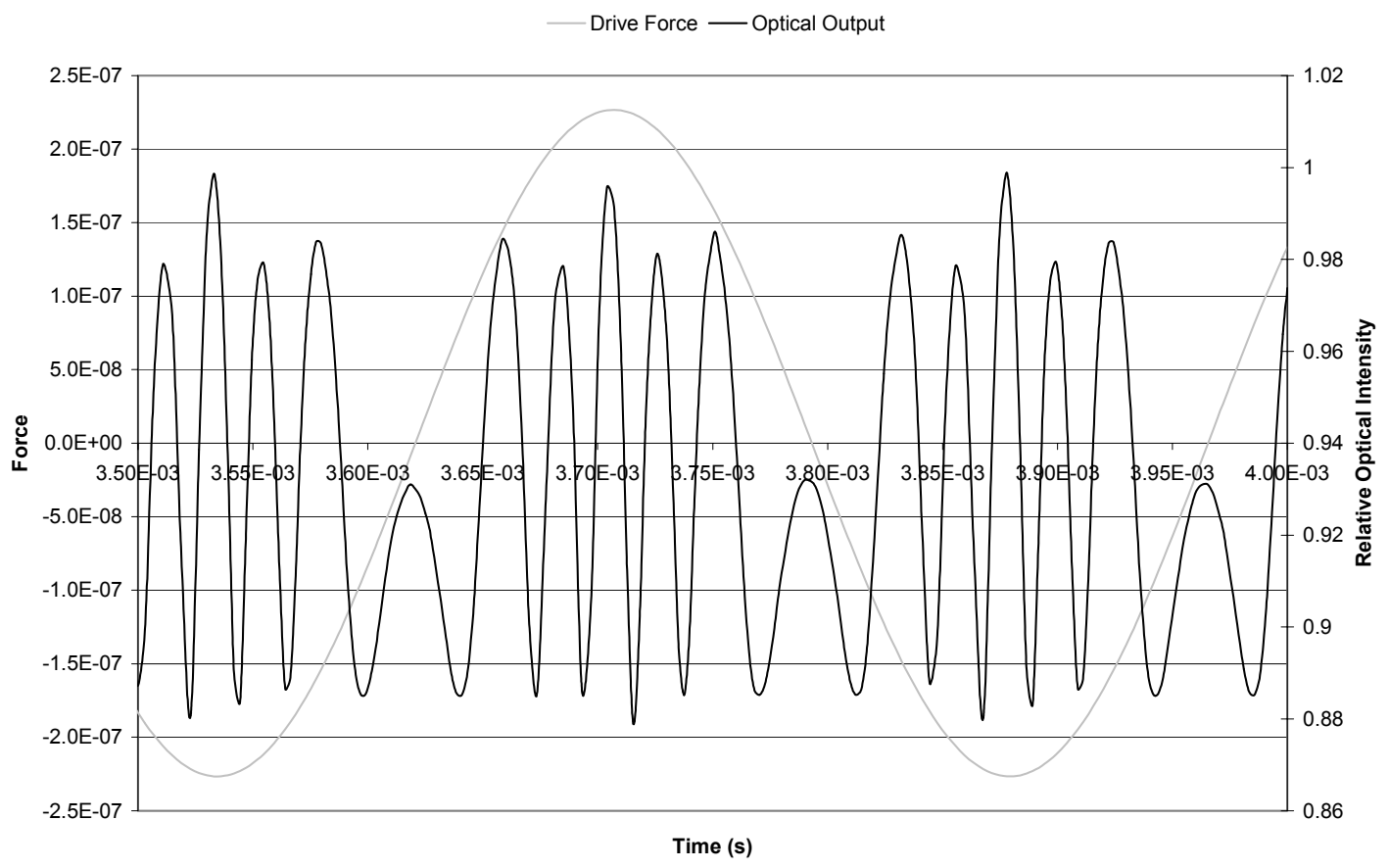

(b)

Experimental Optical Output and Drive Voltage at $2900 \mathrm{~Hz}$

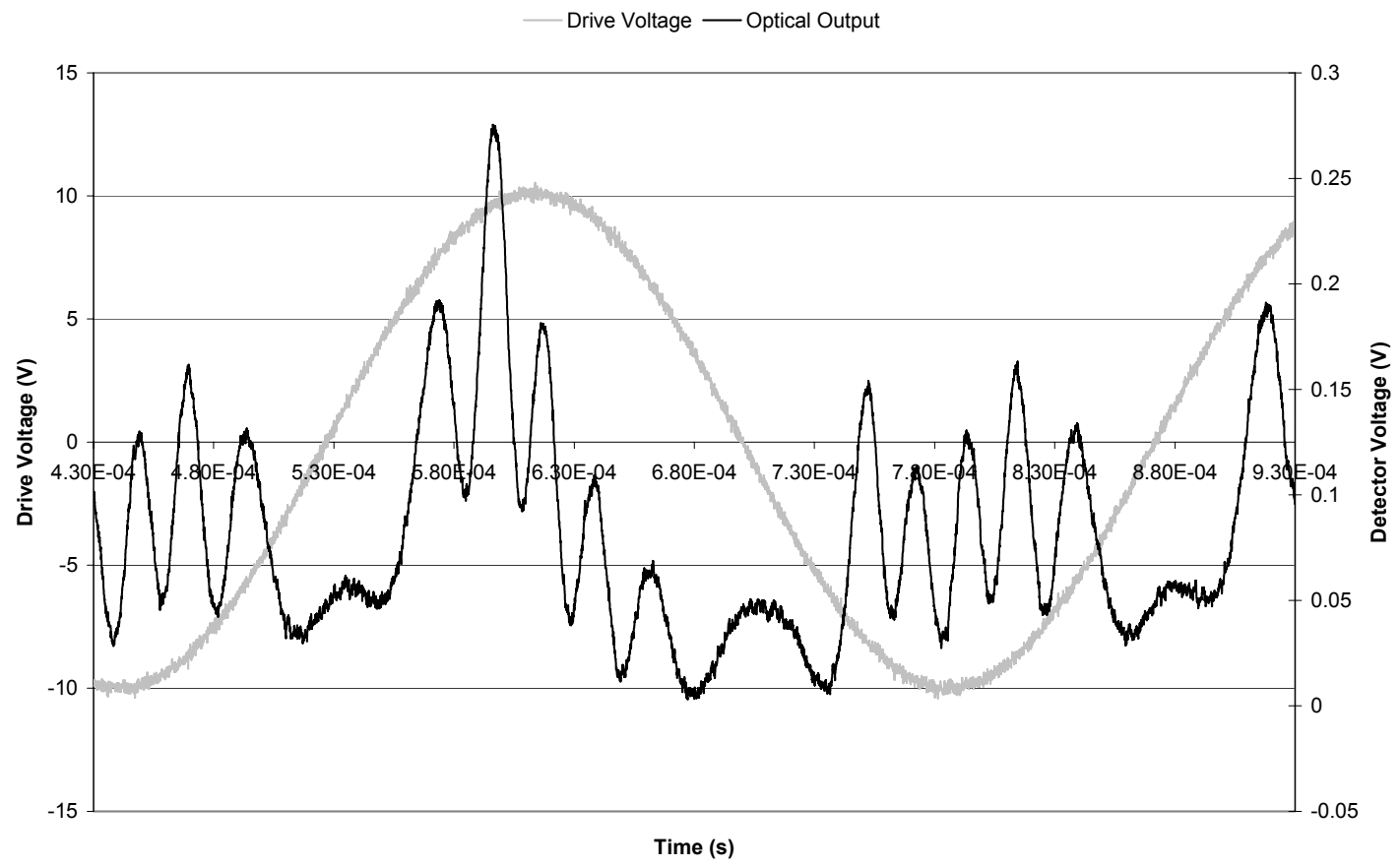

Figure 4.35: Optical output comparison at $2900 \mathrm{~Hz}$ : (a) theoretical results and (b) experimental data. 
(a)

Theoretical Optical Output and Drive Force at $3000 \mathrm{~Hz}$

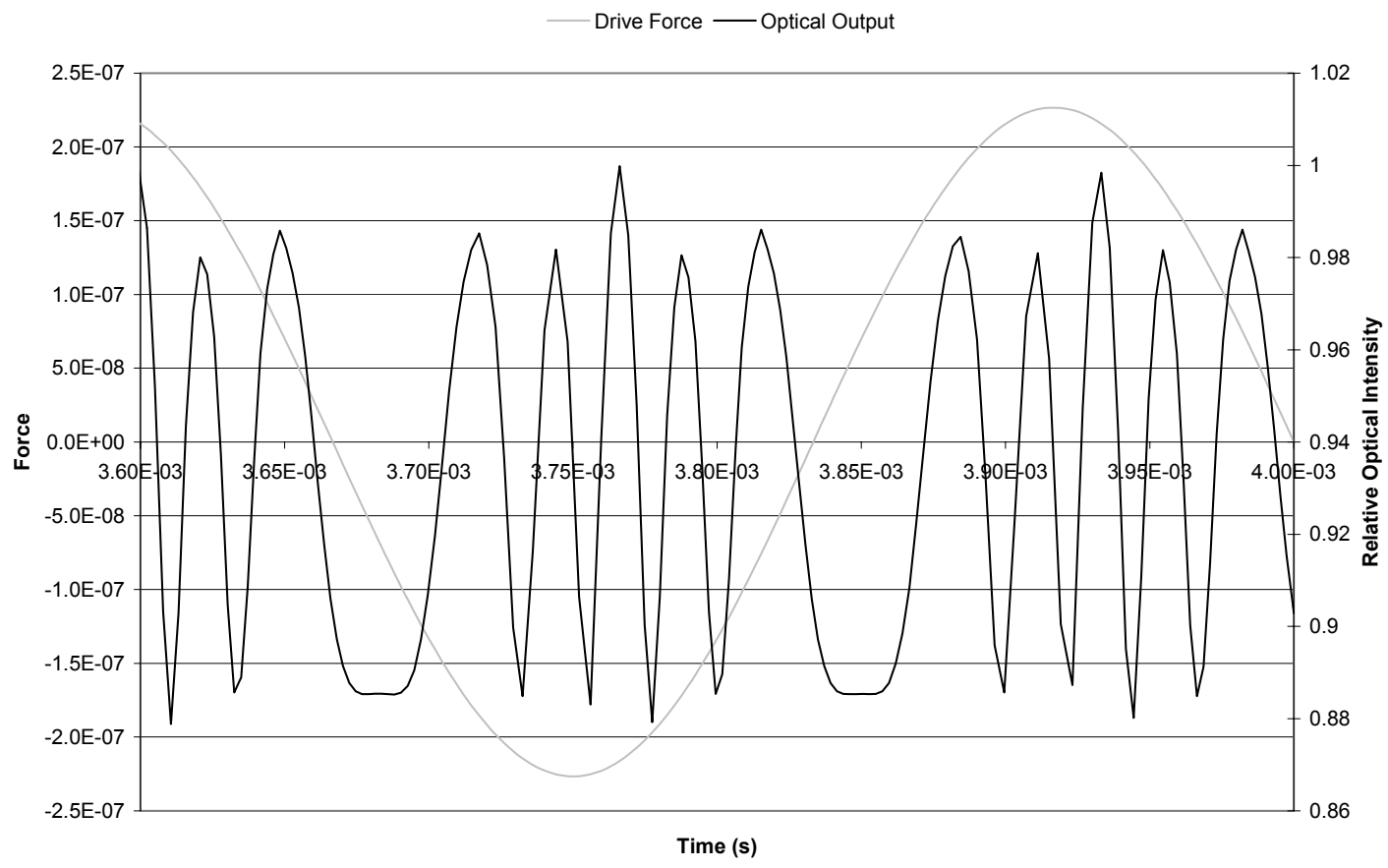

(b)

Experimental Optical Output and Drive Voltage at $3000 \mathrm{~Hz}$

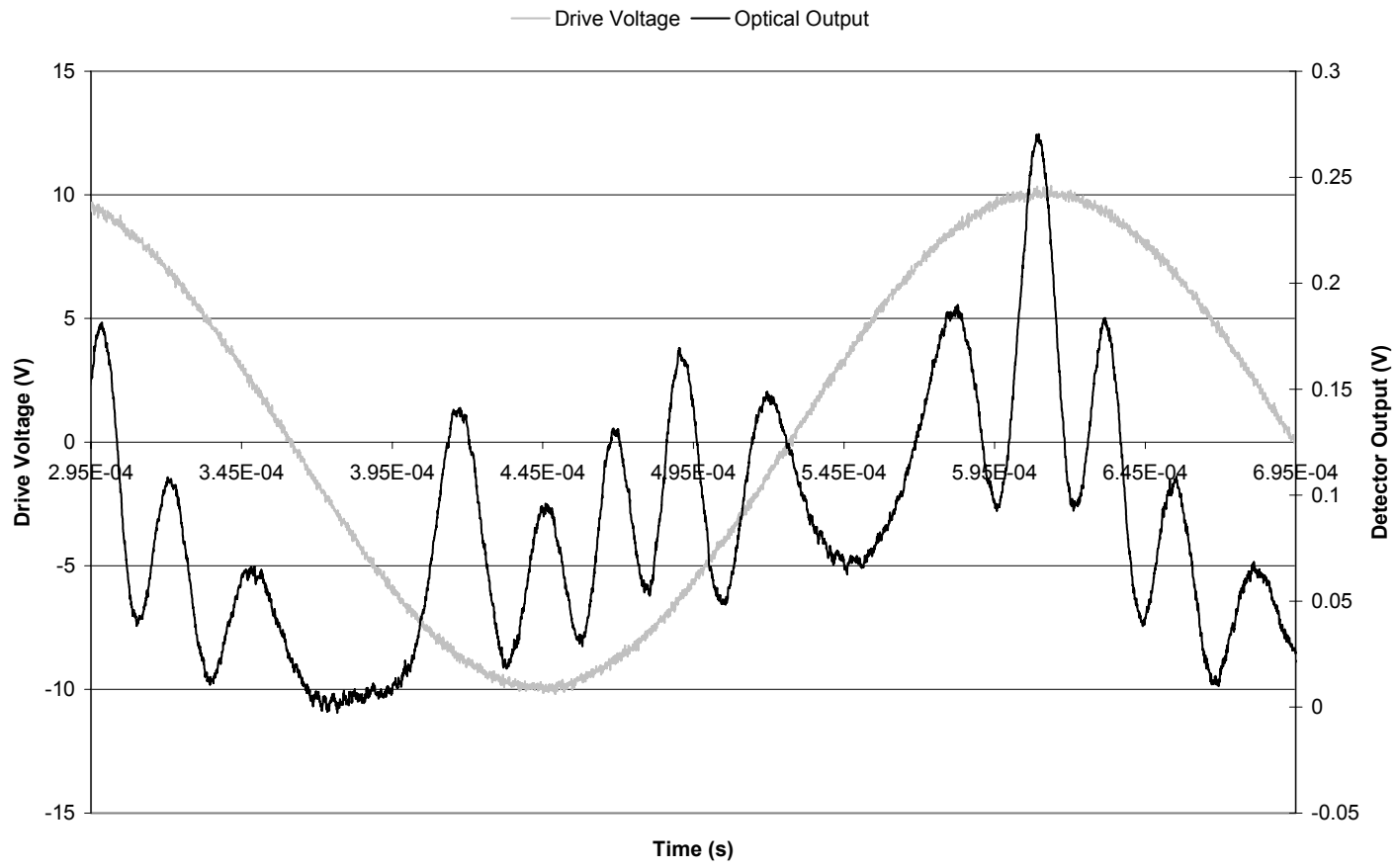

Figure 4.36: Optical output comparison at $3000 \mathrm{~Hz}$ : (a) theoretical results and (b) experimental data. 
(a)

Theoretical Optical Output and Drive Force at $3100 \mathrm{~Hz}$

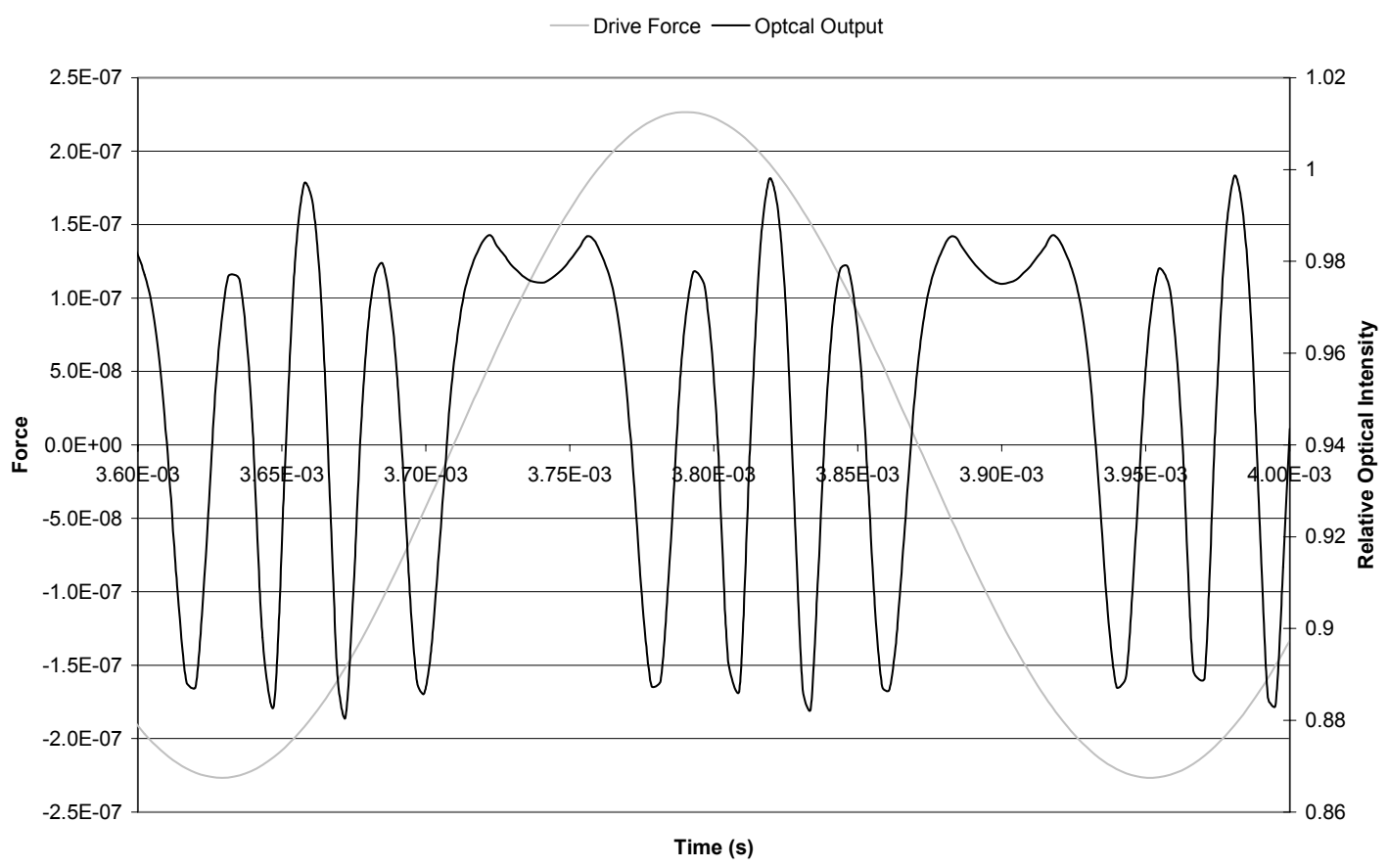

(b)

Experimental Optical Output and Drive Voltage at $3100 \mathrm{~Hz}$

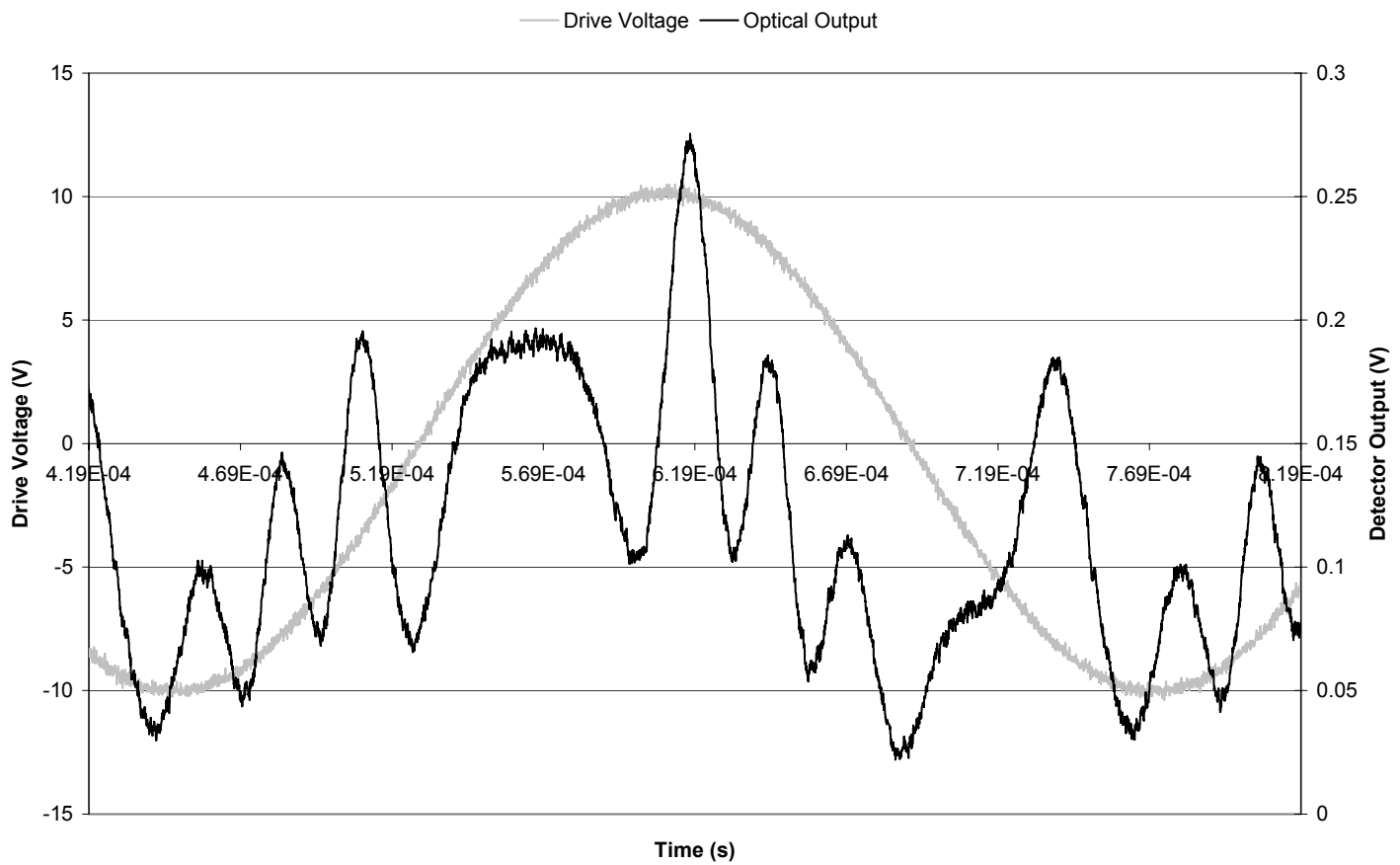

Figure 4.37: Optical output comparison at $3100 \mathrm{~Hz}$ : (a) theoretical results and (b) experimental data. 
(a)

Theoretical Optical Output and Drive Force at $3200 \mathrm{~Hz}$

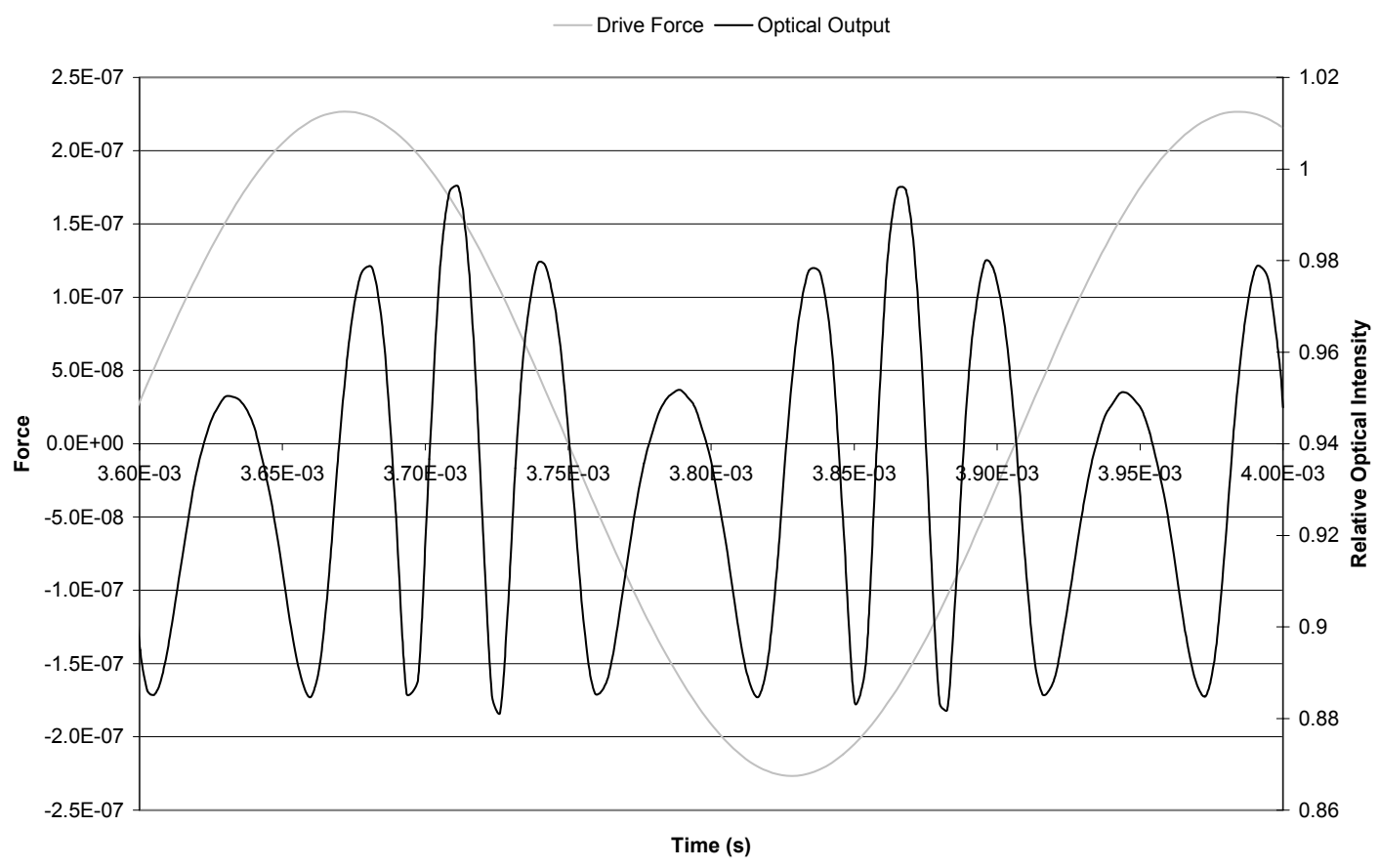

(b)

Experimental Optical Output and Drive Voltage at $3200 \mathrm{~Hz}$

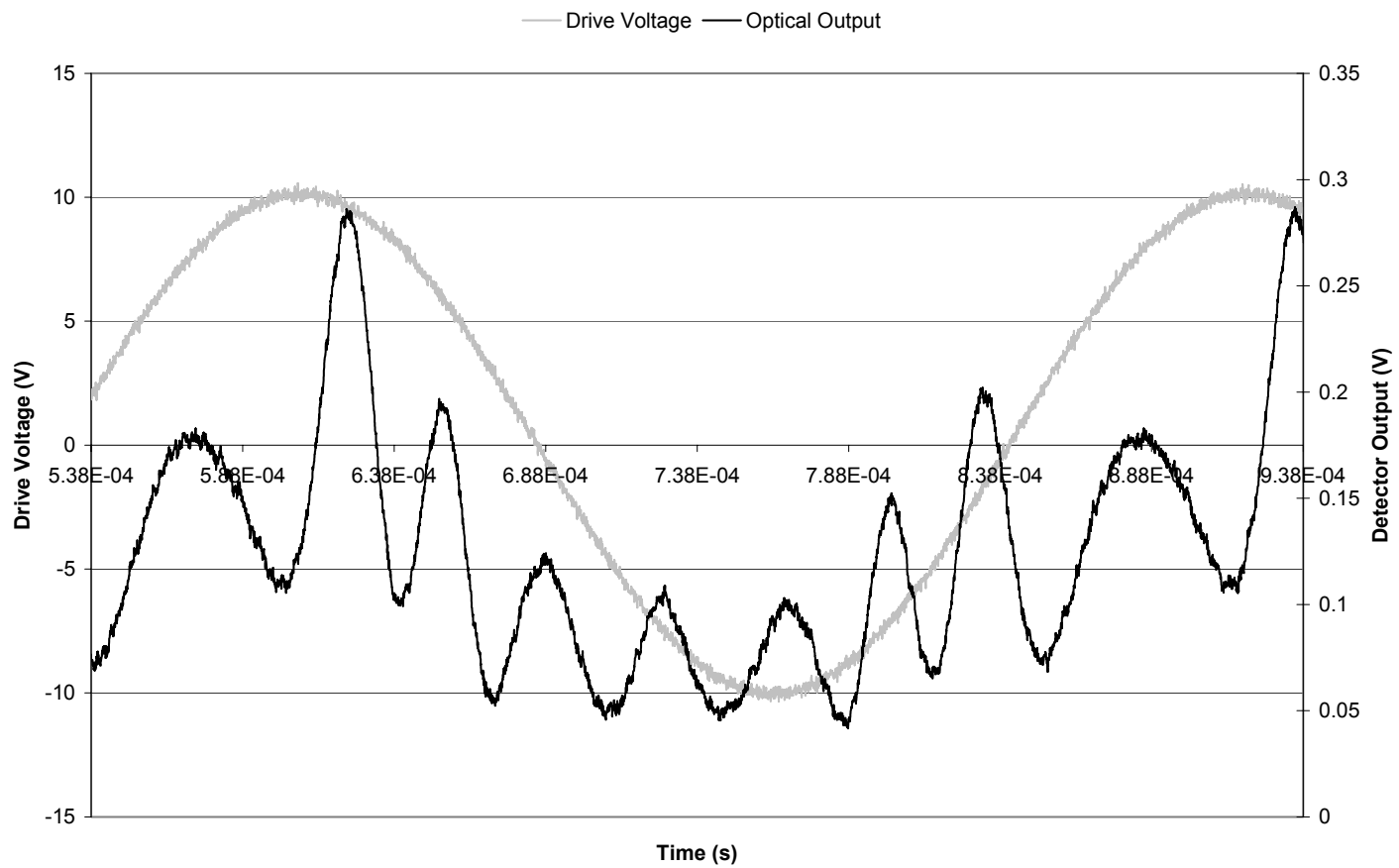

Figure 4.38: Optical output comparison at $3200 \mathrm{~Hz}$ : (a) theoretical results and (b) experimental data. 
(a)

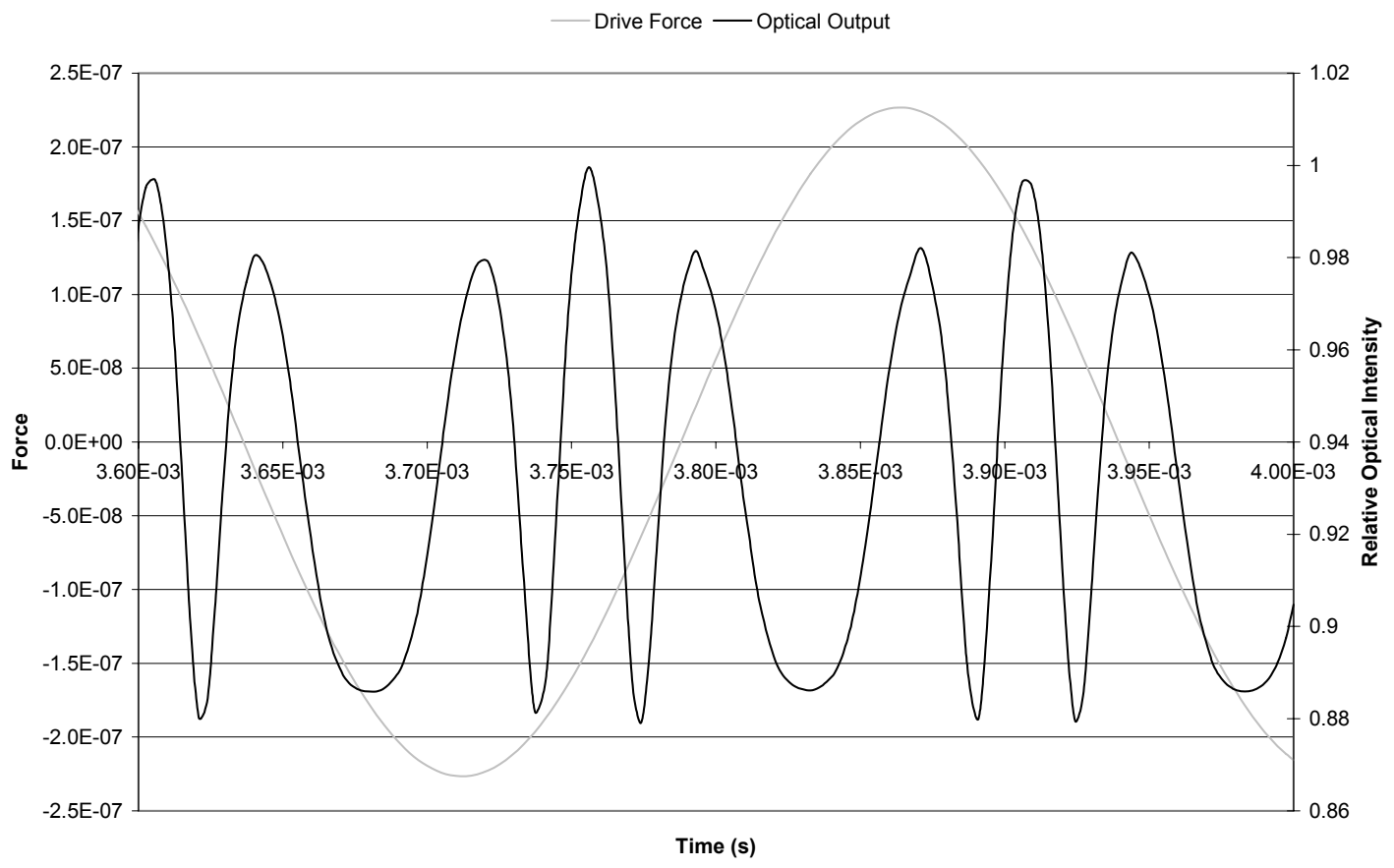

(b)

Experimental Optical Output and Drive Voltage at $3300 \mathrm{~Hz}$

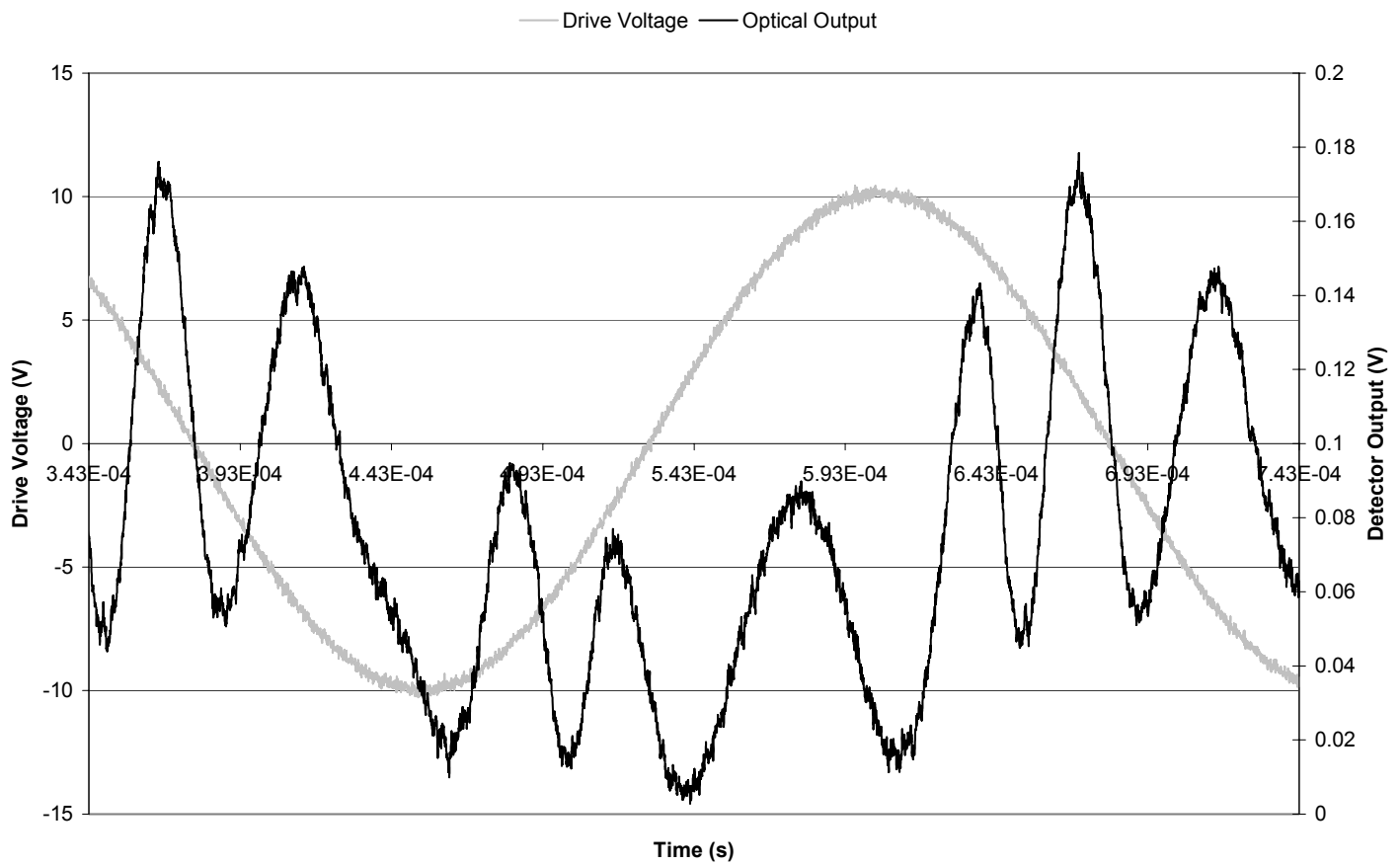

Figure 4.39: Optical output comparison at $3300 \mathrm{~Hz}$ : (a) theoretical results and (b) experimental data. 
(a)

Theoretical Optical Output and Drive Force at $3400 \mathrm{~Hz}$

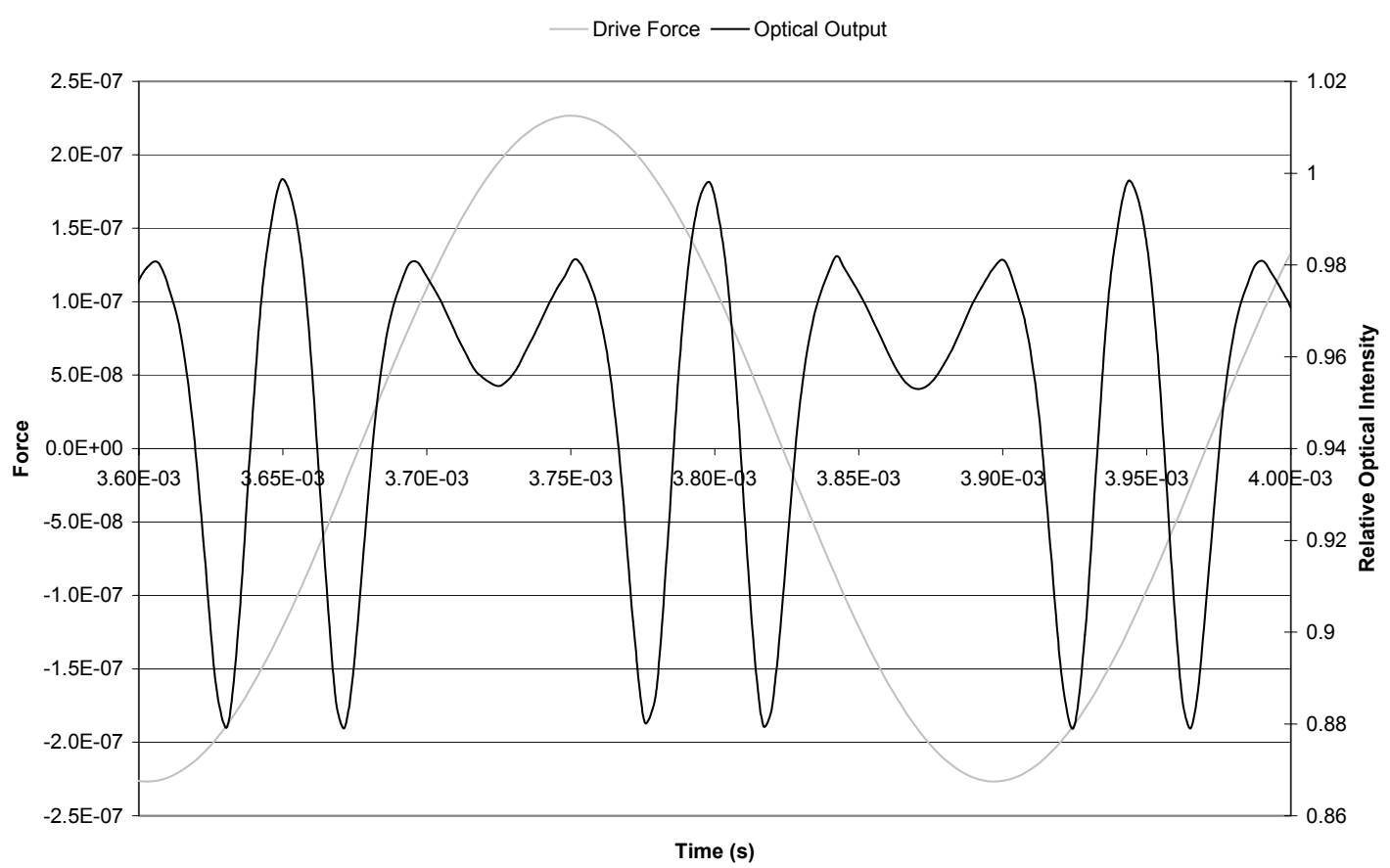

(b)

Experimental Optical Output and Drive Voltage at $3400 \mathrm{~Hz}$

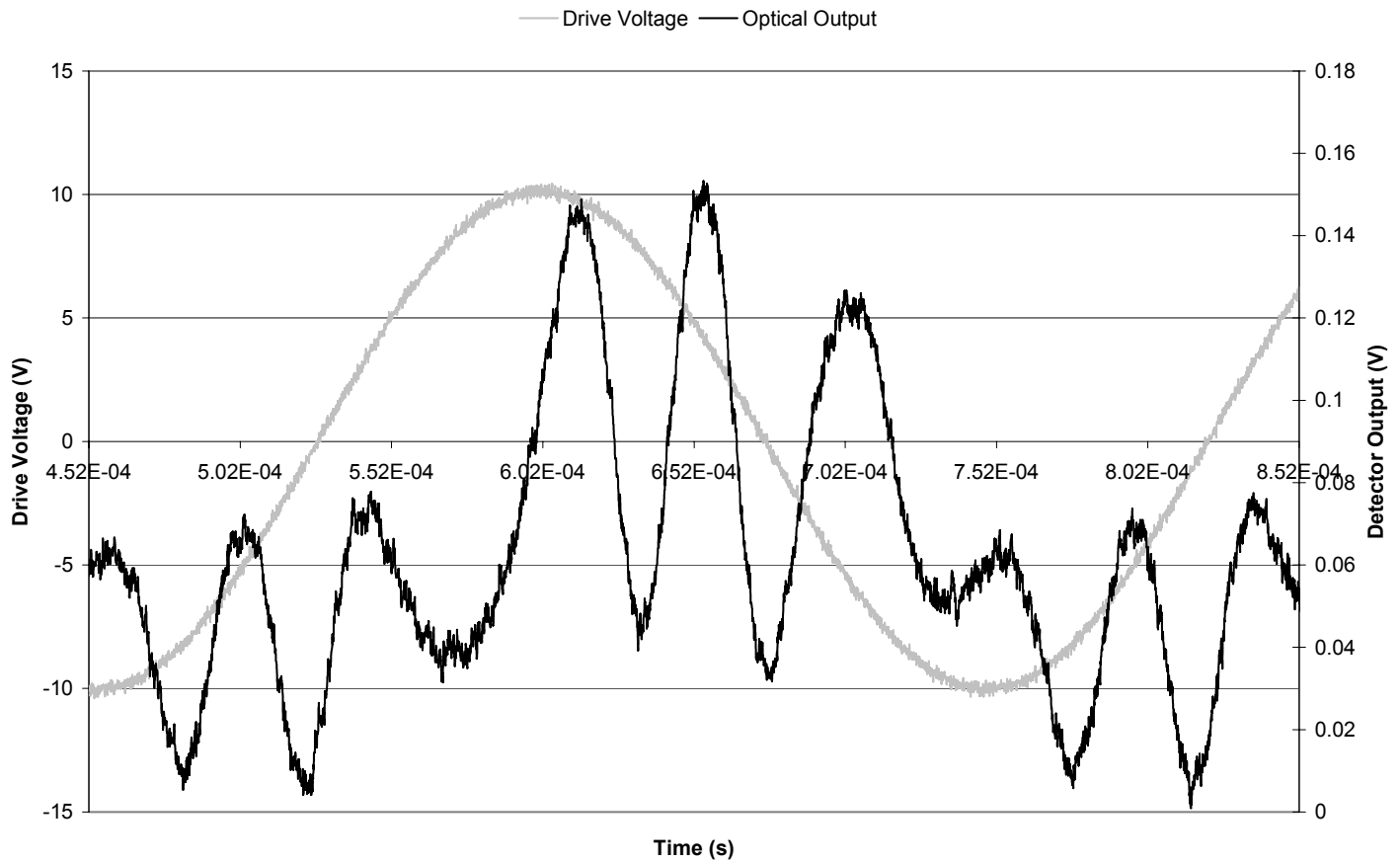

Figure 4.40: Optical output comparison at $3400 \mathrm{~Hz}$ : (a) theoretical results and (b) experimental data. 
(a)

Theoretical Optical Output and Drive Force at $3600 \mathrm{~Hz}$

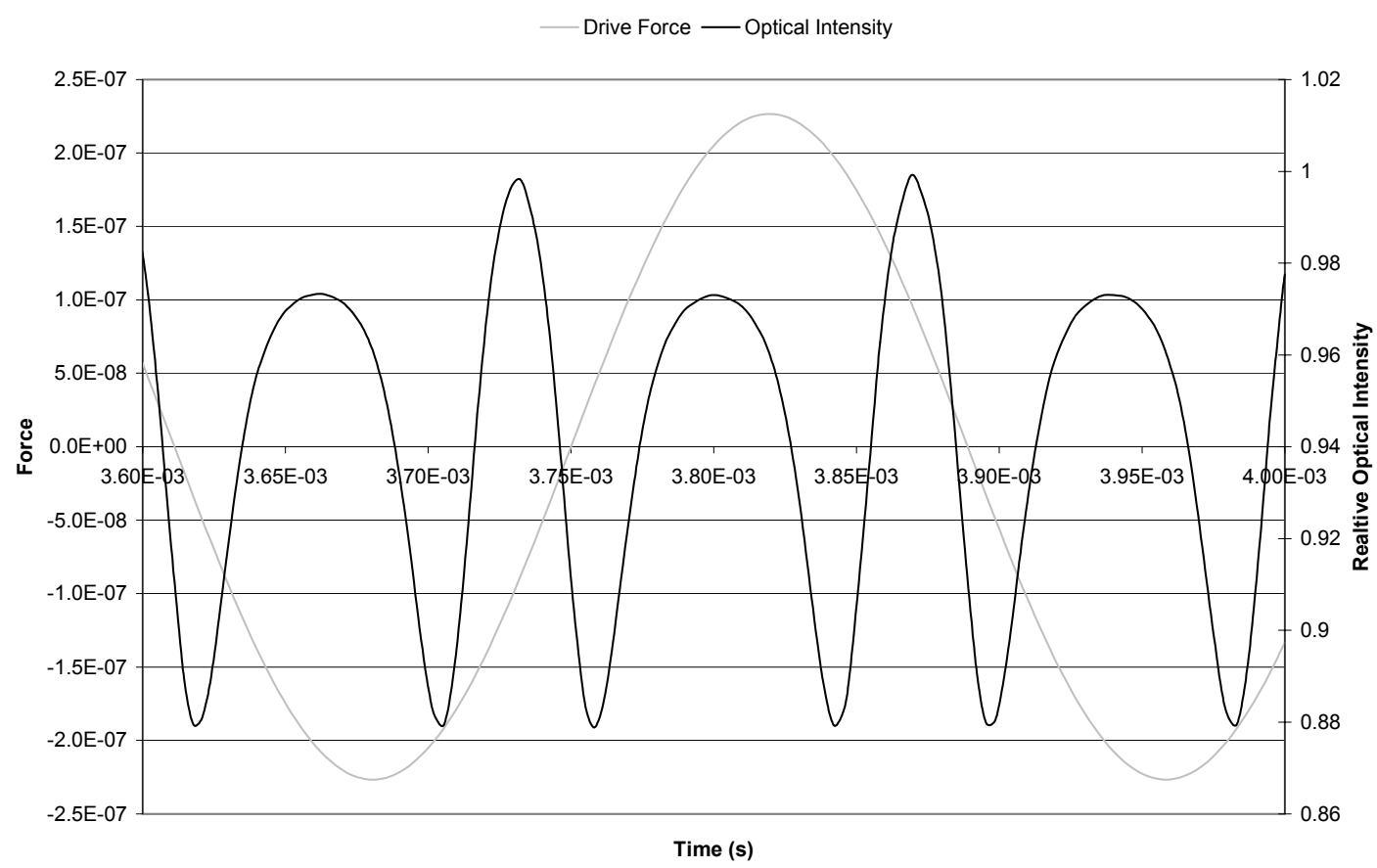

(b)

Experimental Optical Output and Drive Voltage at $3600 \mathrm{~Hz}$

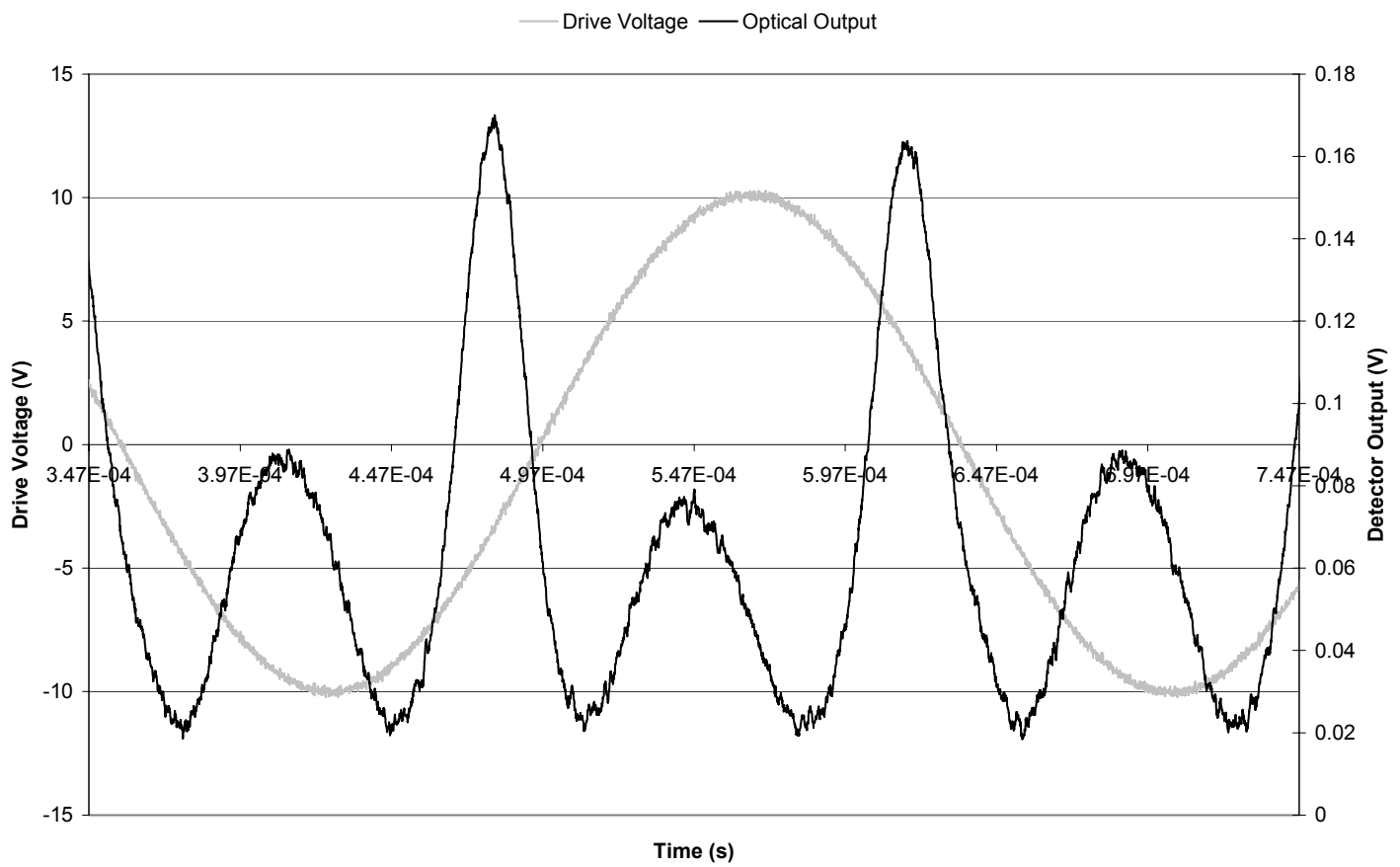

Figure 4.41: Optical output comparison at $3600 \mathrm{~Hz}$ : (a) theoretical results and (b) experimental data. 
(a)

Theoretical Optical Output and Drive Force at $3800 \mathrm{~Hz}$

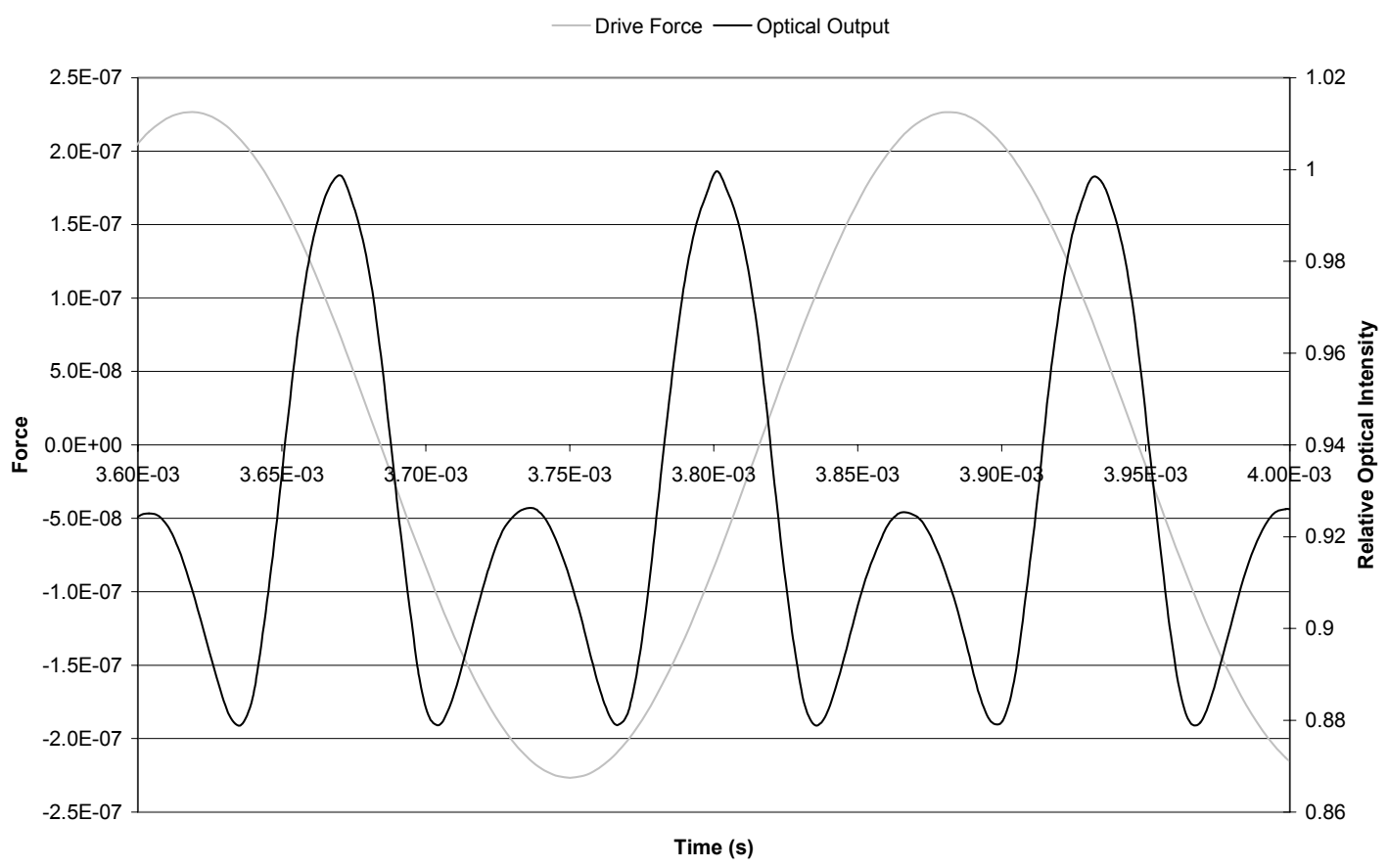

(b)

Experimental Optical Output and Drive Voltage at $3800 \mathrm{~Hz}$

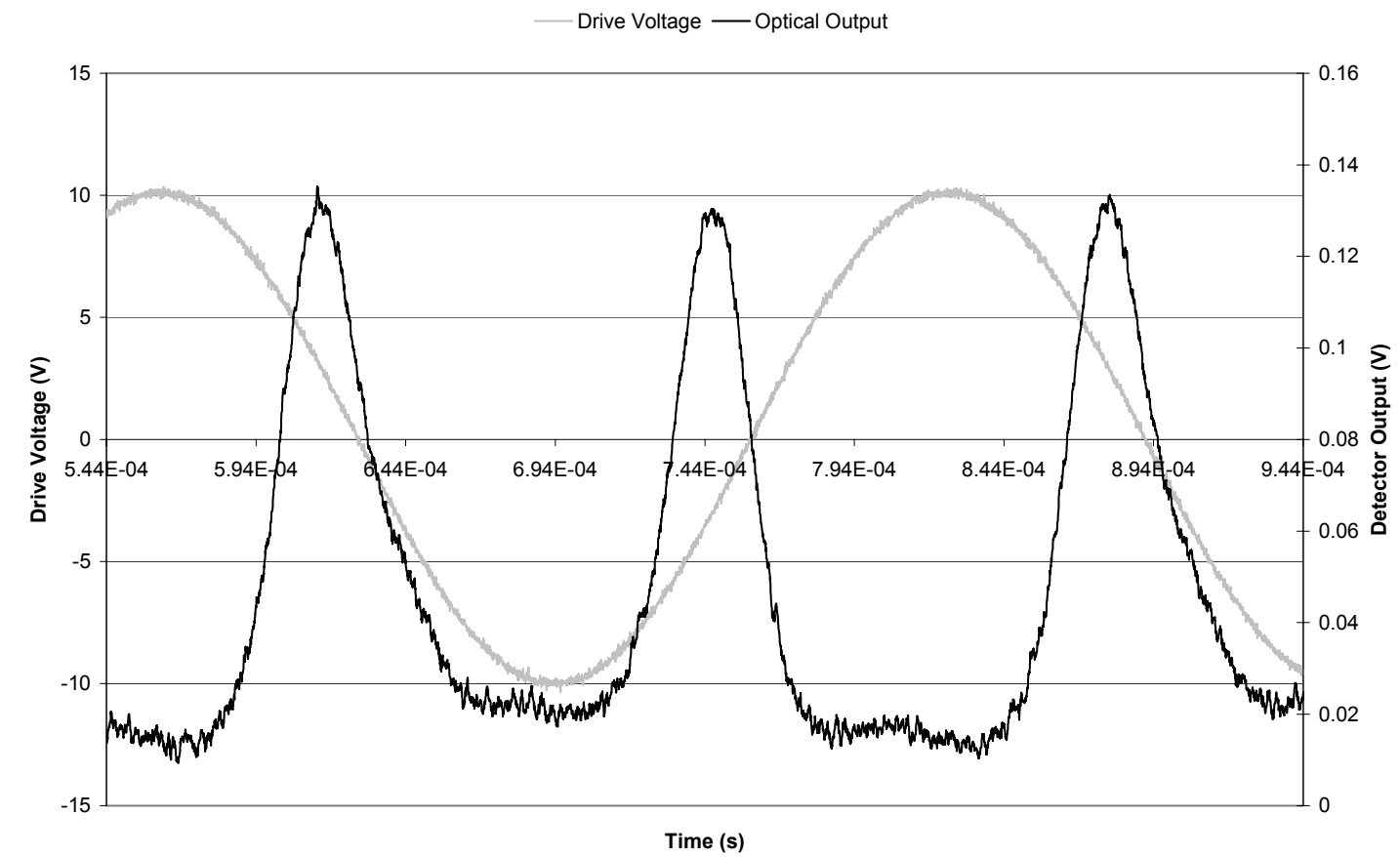

Figure 4.42: Optical output comparison at $3800 \mathrm{~Hz}$ : (a) theoretical results and (b) experimental data. 
(a)

Theoretical Optical Output and Drive Force at $4000 \mathrm{~Hz}$

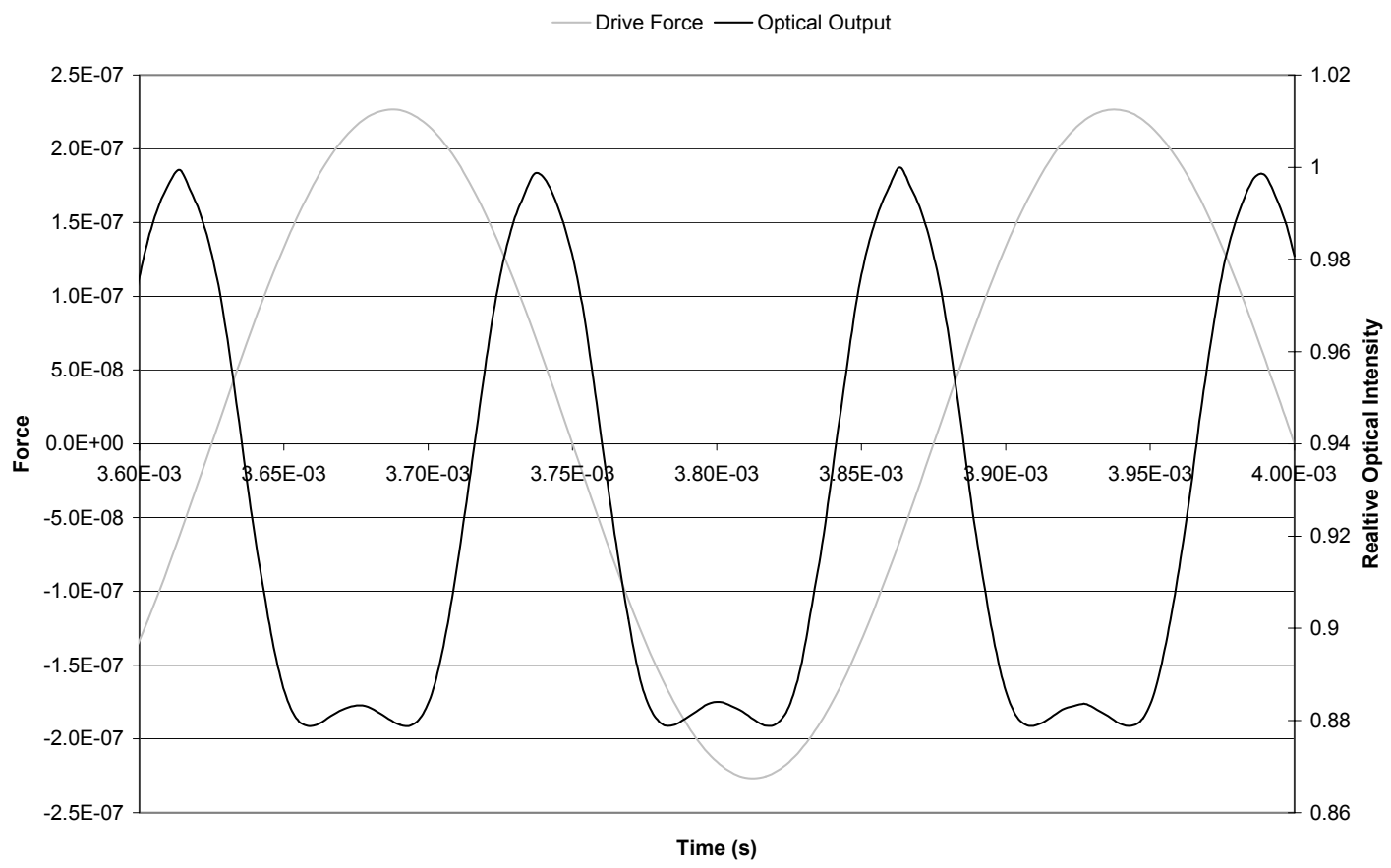

(b)

Experimental Optical Output and Drive Voltage at $4000 \mathrm{~Hz}$

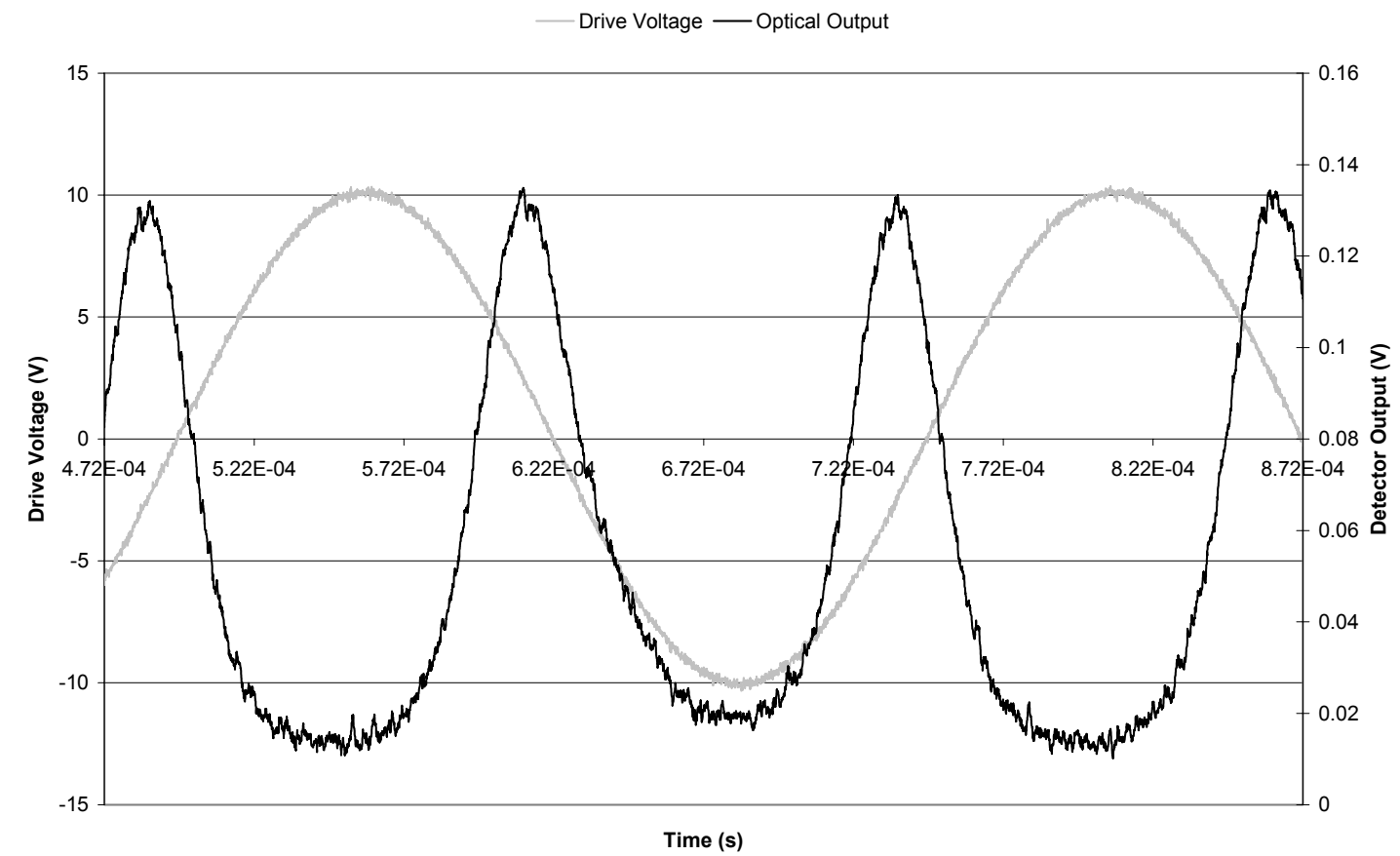

Figure 4.43: Optical output comparison at $4000 \mathrm{~Hz}$ : (a) theoretical results and (b) experimental data. 
(a)

Theoretical Optical Output and Drive Force at $4500 \mathrm{~Hz}$

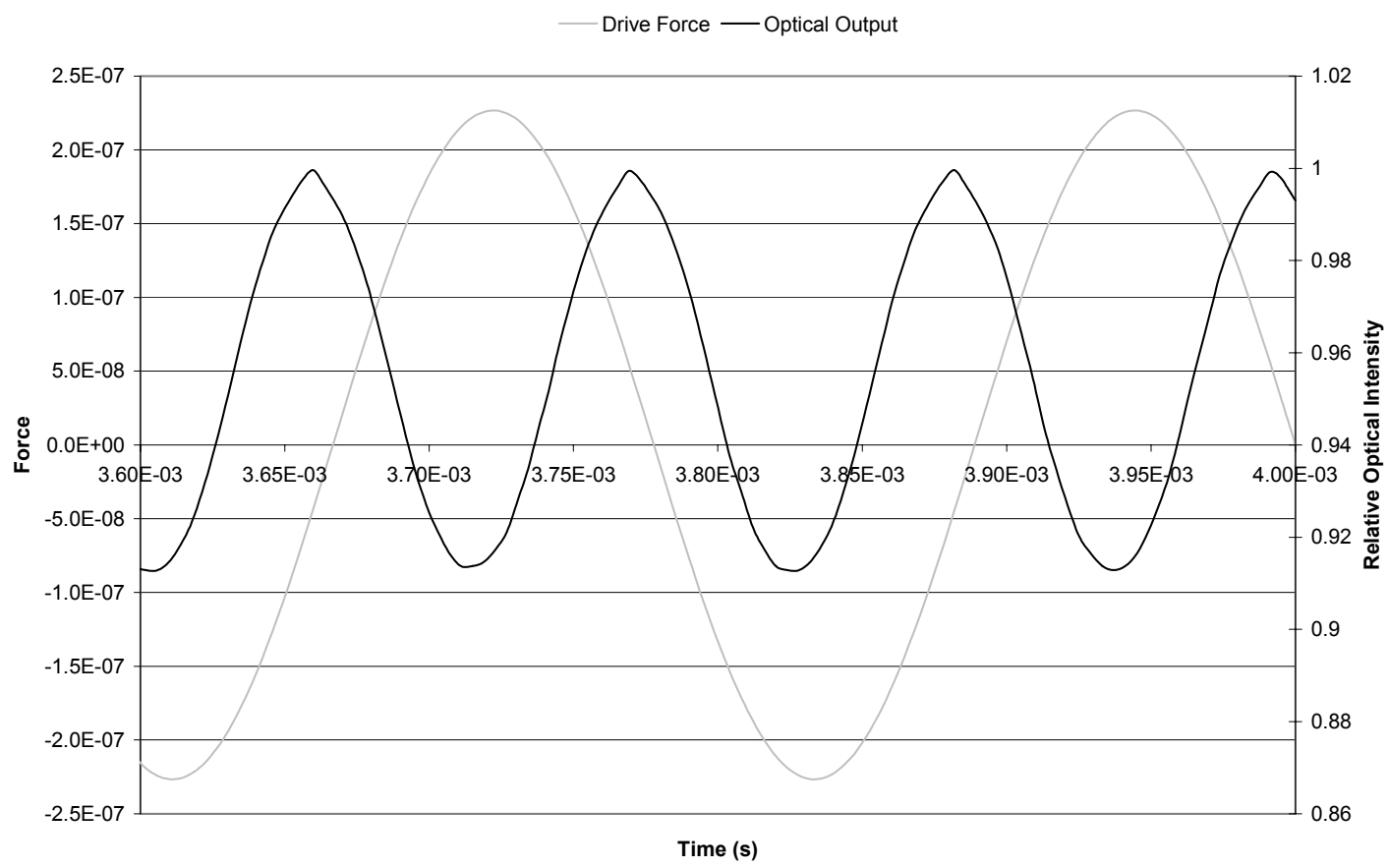

(b)

Experimental Optical Output and Drive Voltage at $4500 \mathrm{~Hz}$

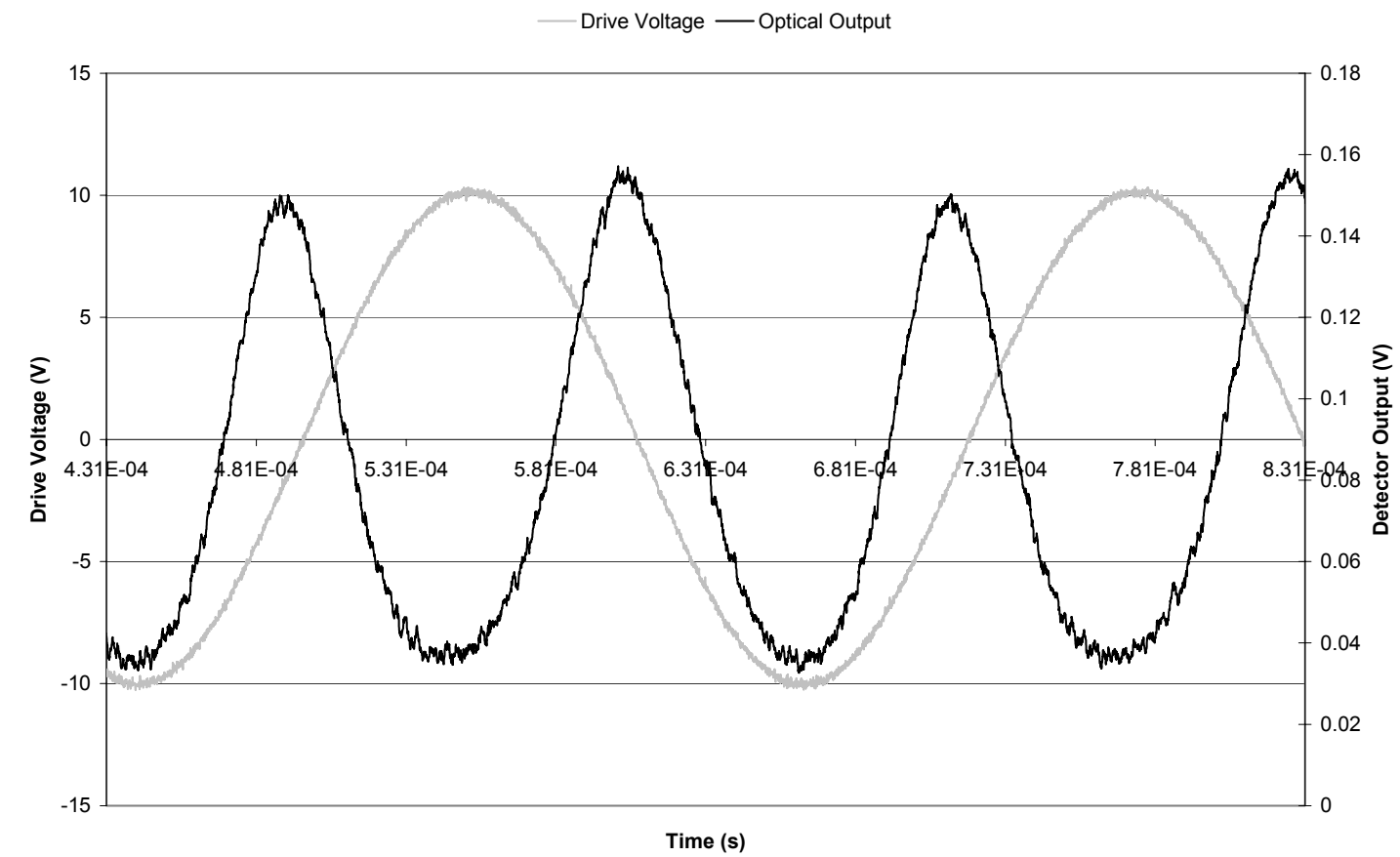

Figure 4.44: Optical output comparison at $4500 \mathrm{~Hz}$ : (a) theoretical results and (b) experimental data. 
(a)

Theoretical Optical Output and Drive Force at $5000 \mathrm{~Hz}$

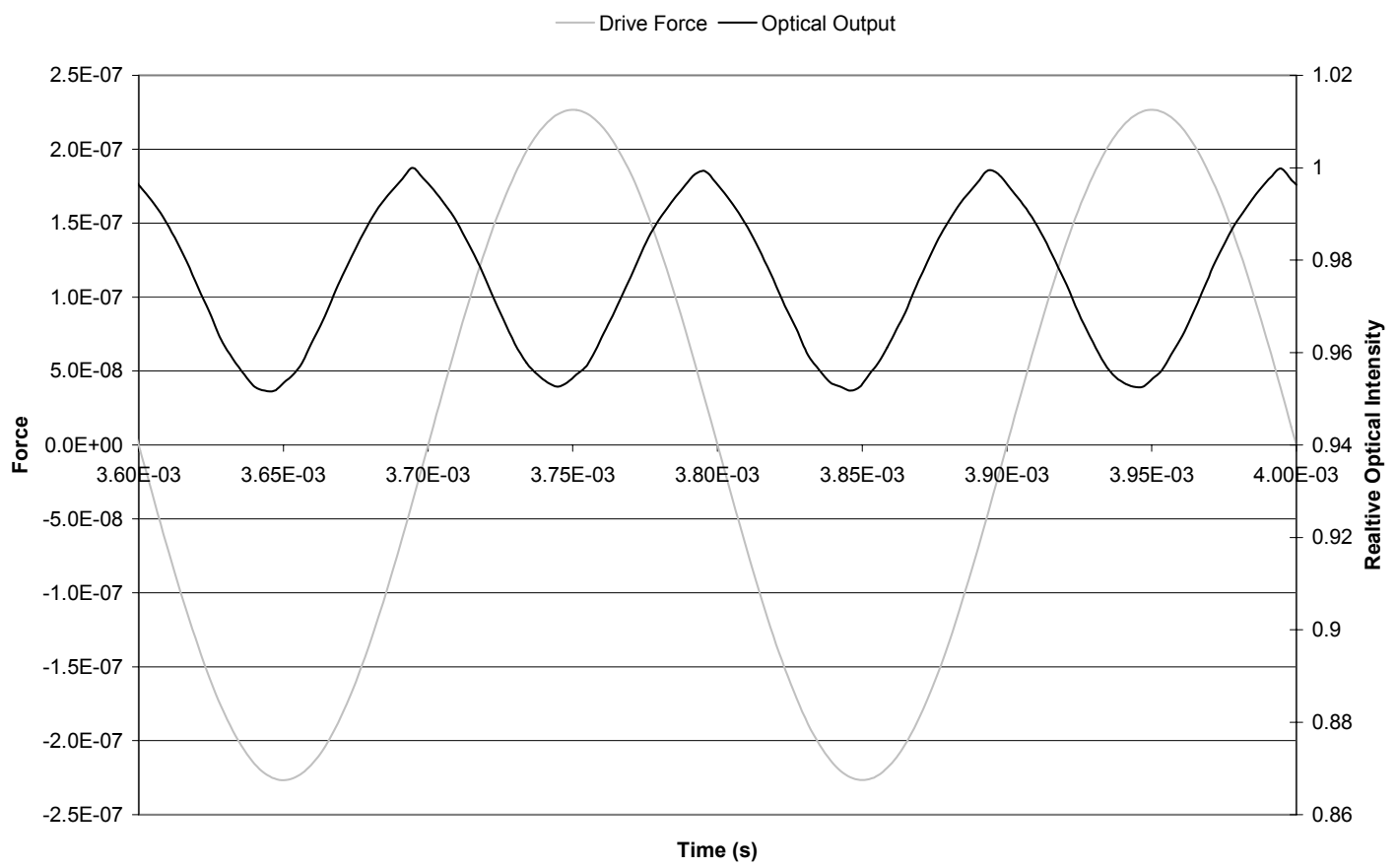

(b)

Experimental Optical Output and Drive Voltage at $5000 \mathrm{~Hz}$

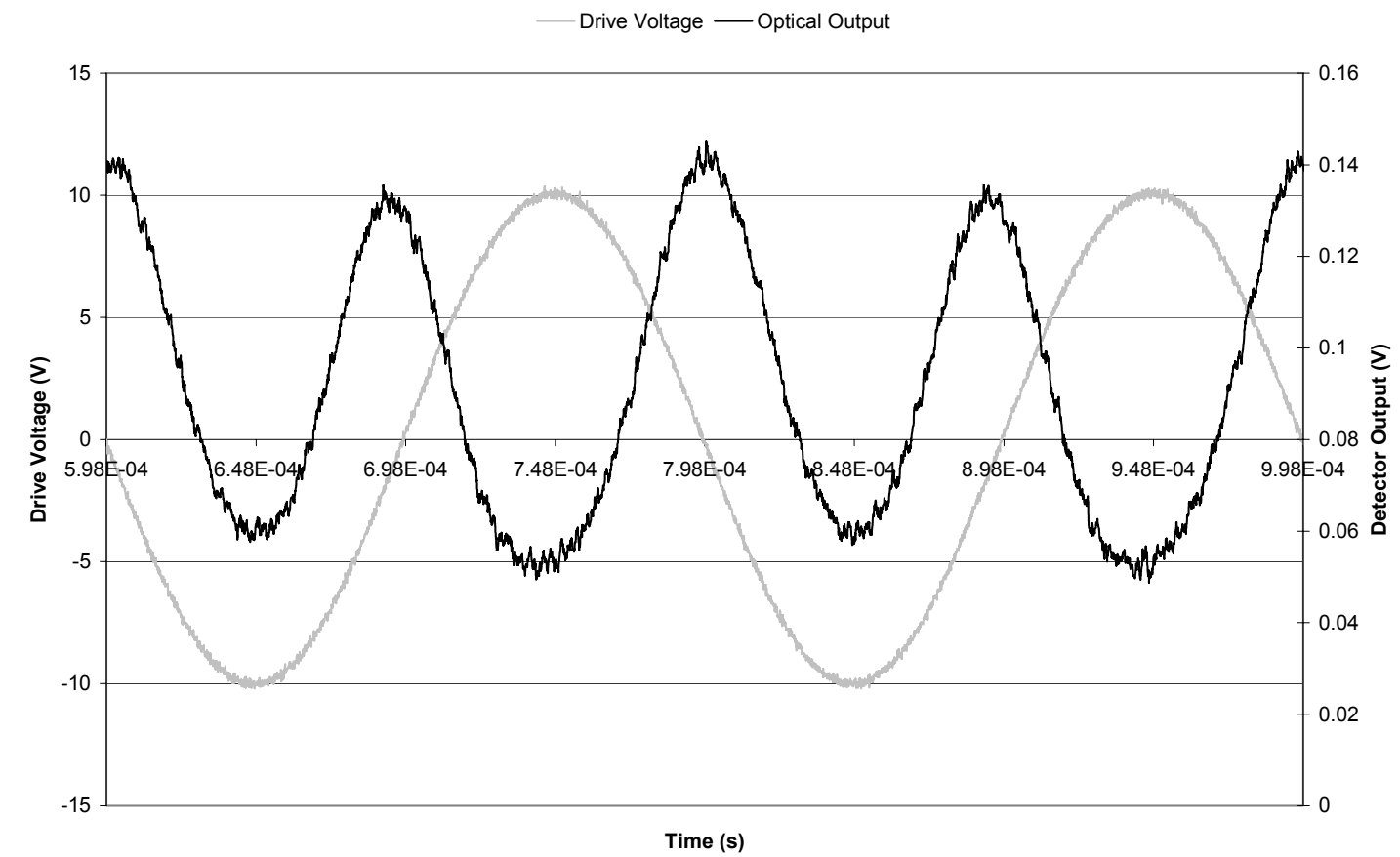

Figure 4.45: Optical output comparison at $5000 \mathrm{~Hz}$ : (a) theoretical results and (b) experimental data. 
It can be seen in the comparison of theoretical and actual data that optical outputs match in both number of major peaks and peak width. The phase differential between sinusoidal drive voltage and output optical signal, due to the behavior of a forced harmonic oscillator with damping, is in agreement as well. Peak width variation is in accordance with the velocity of the translation stage at different positions over its range of motion. Near the maximum range of motion, the stage moves slowest, causing broadened intensity peaks. Near the zero position, the stage moves fastest, resulting in narrowed peaks. Slight variations in theoretical and optical data at 3600 and $3800 \mathrm{~Hz}$ are mainly due to asymmetry caused by the motion resolution of the motorized translation stages used to position the device grating in the path of the probe beam.

This data confirms the optical output waveform resolution of $4 \mu \mathrm{m}$ peak to peak and 2 $\mu \mathrm{m}$ peak to valley when examining theoretical stage translation determined by system model parameters extracted from previous experimental results and grating signal experimental output data. Values for displacement in one direction are listed in Table 4.1.

Table 4.1: Comparison of theoretical and experimentally determined displacement values.

\begin{tabular}{||c||c|c||}
\hline Frequency (Hz) & $\begin{array}{c}\text { Theoretical } \\
\text { Displacement }(\boldsymbol{\mu} \mathbf{m})\end{array}$ & $\begin{array}{c}\text { Experimentally } \\
\text { Determined } \\
\text { Displacement }(\boldsymbol{\mu} \mathbf{m})\end{array}$ \\
\hline \hline 1000 & 2.37 & $>2$ \\
\hline 1500 & 2.84 & $>2$ \\
\hline 2000 & 3.83 & $<4$ \\
\hline 2200 & 4.56 & $>4$ \\
\hline 2400 & 5.92 & 6 \\
\hline 2600 & 7.84 & 8 \\
\hline 2700 & 9.23 & 10 \\
\hline 2800 & 10.4 & 10 \\
\hline 2900 & 10.7 & $>10$ \\
\hline 3000 & 9.95 & 10 \\
\hline 3100 & 8.46 & 8 \\
\hline 3200 & 7.03 & 8 \\
\hline 3300 & 5.84 & 6 \\
\hline 3400 & 4.84 & $>4$ \\
\hline 3600 & 3.63 & $<4$ \\
\hline 3800 & 2.8 & 2 \\
\hline 4000 & 2.27 & 2 \\
\hline 4500 & 1.48 & 2 \\
\hline 5000 & 1.07 & 2 \\
\hline
\end{tabular}


This table shows that theoretical and experimental displacement values have a high degree of correlation, and the resonant frequency of $2900 \mathrm{~Hz}$ clearly visible. This value falls within the calculated range frequencies for this device given in Table 3.3. Although the experimental output has a resolution of only $2 \mu \mathrm{m}$, secondary peaks significantly lower in amplitude than the normal optical output intensity are present in the experimental data, indicating motion between 0 and $2 \mu \mathrm{m}$, a value that falls below the resolution of the optical signal. Greater-than or less-than symbols in the experimental displacement column of Table 4.1 give a qualitative assessment of the amplitude of motion below the resolution of waveform.

\section{Secondary Signal Frequency}

For frequencies within the range of 2.4-3.4 kHz, experimental data shows the optical output having a secondary frequency close to that of the drive frequency. Two possible explanations for this are vertical stage motion and the interaction of the beam with nongrating areas of the stage.

Using the reflectance calculation given in Equation 2.1-19, Figure 4.46 illustrates how transmitted intensity changes as the oxide gap is varied from 0 to $2 \mu \mathrm{m}$ (Appendix A, 'reflect.m'). 


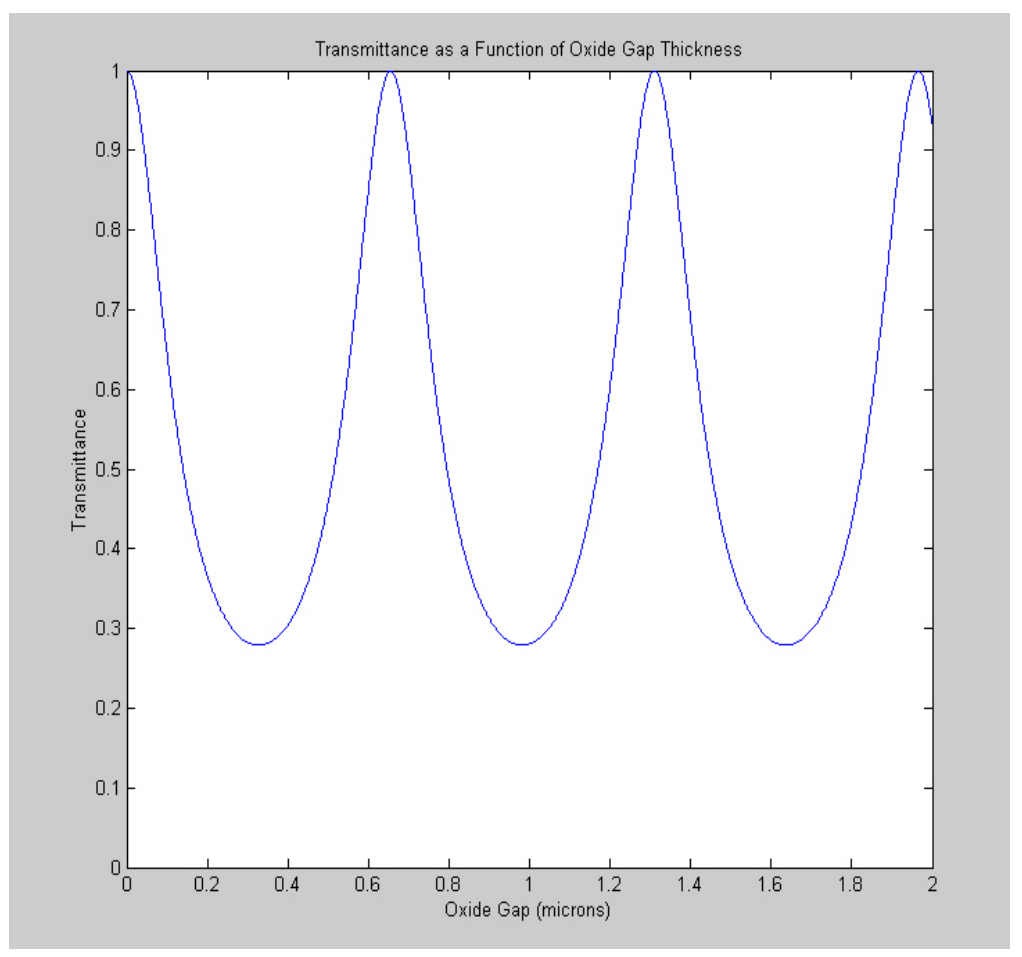

Figure 4.46: Change in transmission due to variation in oxide gap thickness.

At gap distances equal to odd multiples of quarter wavelengths of the probe beam, the gap acts as a Fabry-Perot etalon, or resonance cavity, as evident in the low transmission intensities at these gap values. At even multiples of quarter wavelengths, the gap acts as an anti-reflection layer, theoretically resulting in transmittance values of unity.

A sinusoidal change in transmitted intensity due to vertical motion will only occur if the motion is varied in the regions between maxim and minima on the transmission plot. The most reasonable assumption of where this motion takes place is between 1.7 and 1.9 microns. This allows for some initial sag in the vertical stage position due to its effective mass, and is a small enough value to allow the comb fingers to remain fully coupled. Figure 4.47 shows how transmitted intensity changes due to $0.2 \mu \mathrm{m}$ of vertical motion at a frequency of $2.9 \mathrm{kHz}$ (Appendix A, 'vertsim1d.m'). This frequency is where the experimental optical output shows the highest amplitude of secondary signal. 


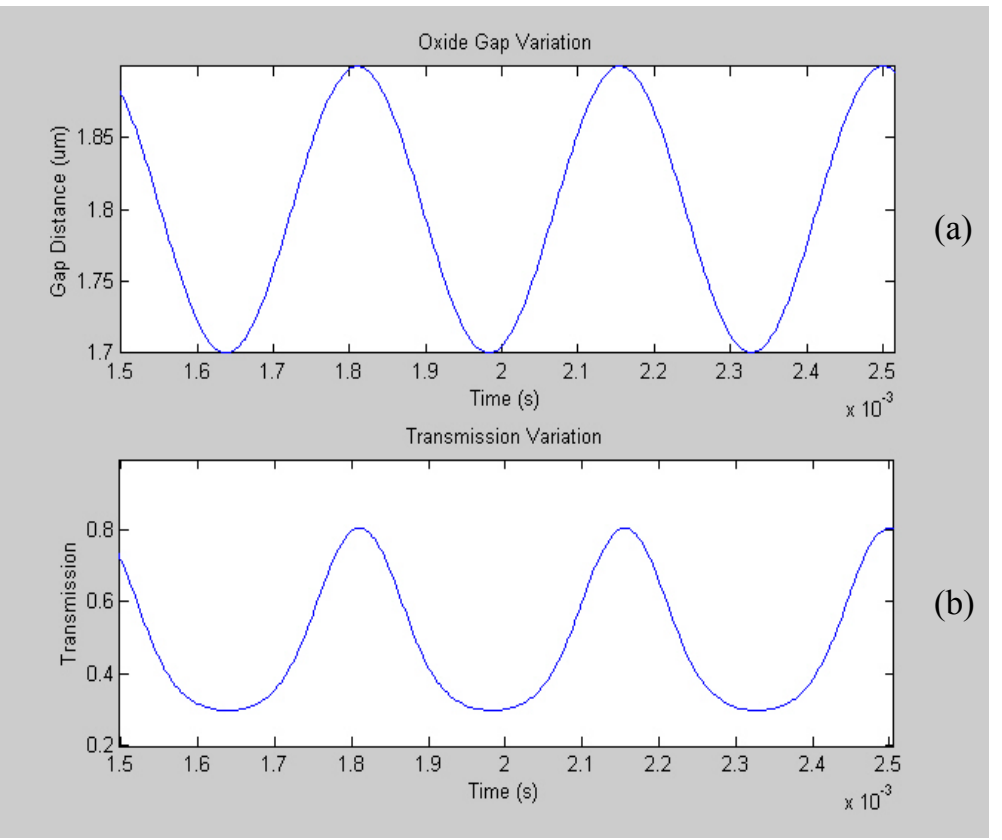

Figure 4.47: Oxide gap variation at $2.9 \mathrm{kHz}$ (a) and the resulting transmission intensity change (b).

The $0.2 \mu \mathrm{m}$ change in vertical stage position causes the theoretical through-wafer transmission intensity to change from 30 to $80 \%$. This change in transmitted intensity can be added to the theoretical output waveform to simulate the secondary frequency of the experimental output, as illustrated in Figure 4.48.

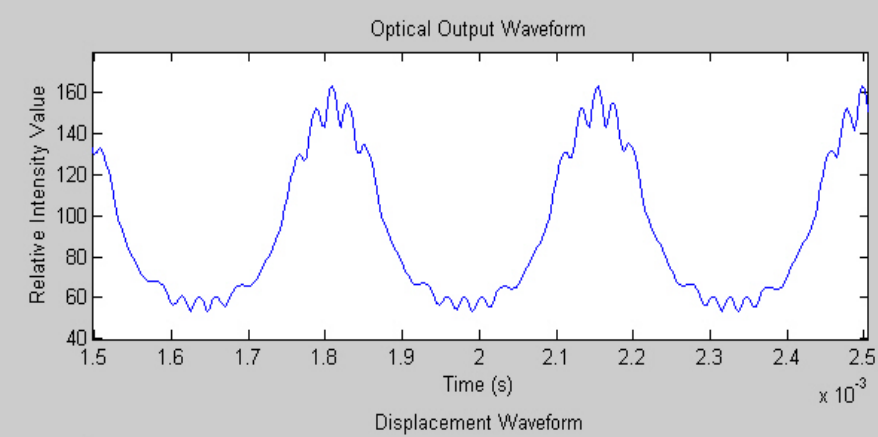

(a)

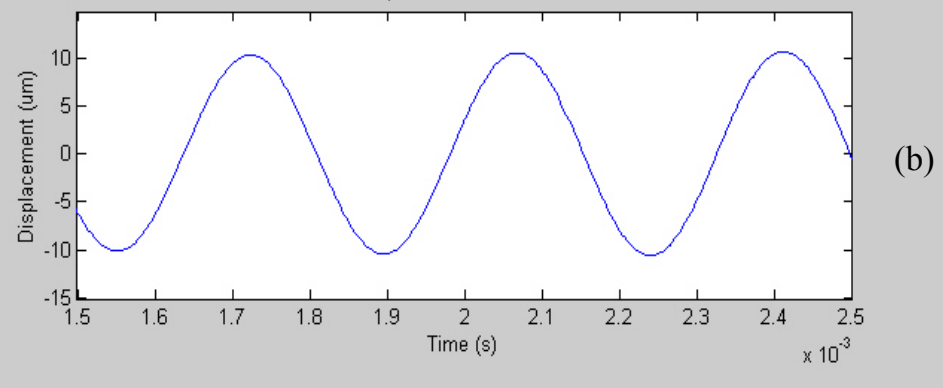

Figure 4.48: The theoretical optical output waveform resulting from vertical stage motion (a) and lateral displacement (b). 
This result does show a secondary signal frequency due to vertical stage motion, but it is significantly larger in amplitude than in experimental data. To achieve the smaller amount of secondary frequency amplitude present in experimental data, the vertical gap change must be reduced to only $0.01 \mu \mathrm{m}$ in the simulation. The resonant frequency of the flexures in the $z$ direction theoretically should be much higher than $x$-direction resonance due to the stiffness of the folded flexure design, causing the secondary signal frequency to be different than that of the drive voltage. These differences suggest that the secondary frequency must come from another source.

Another cause of the secondary frequency in the experimental data could be interaction of the probe beam with non-grating (solid) areas on the device stage. Large displacements at high frequencies or angular misalignment of the grating with respect to direction of motion, along with a large spot sizes $20 \mu \mathrm{m}$ or larger could cause a secondary sinusoidal frequency in the optical output waveform that has the same frequency as the drive voltage. Figure 4.49 illustrates this assumption.
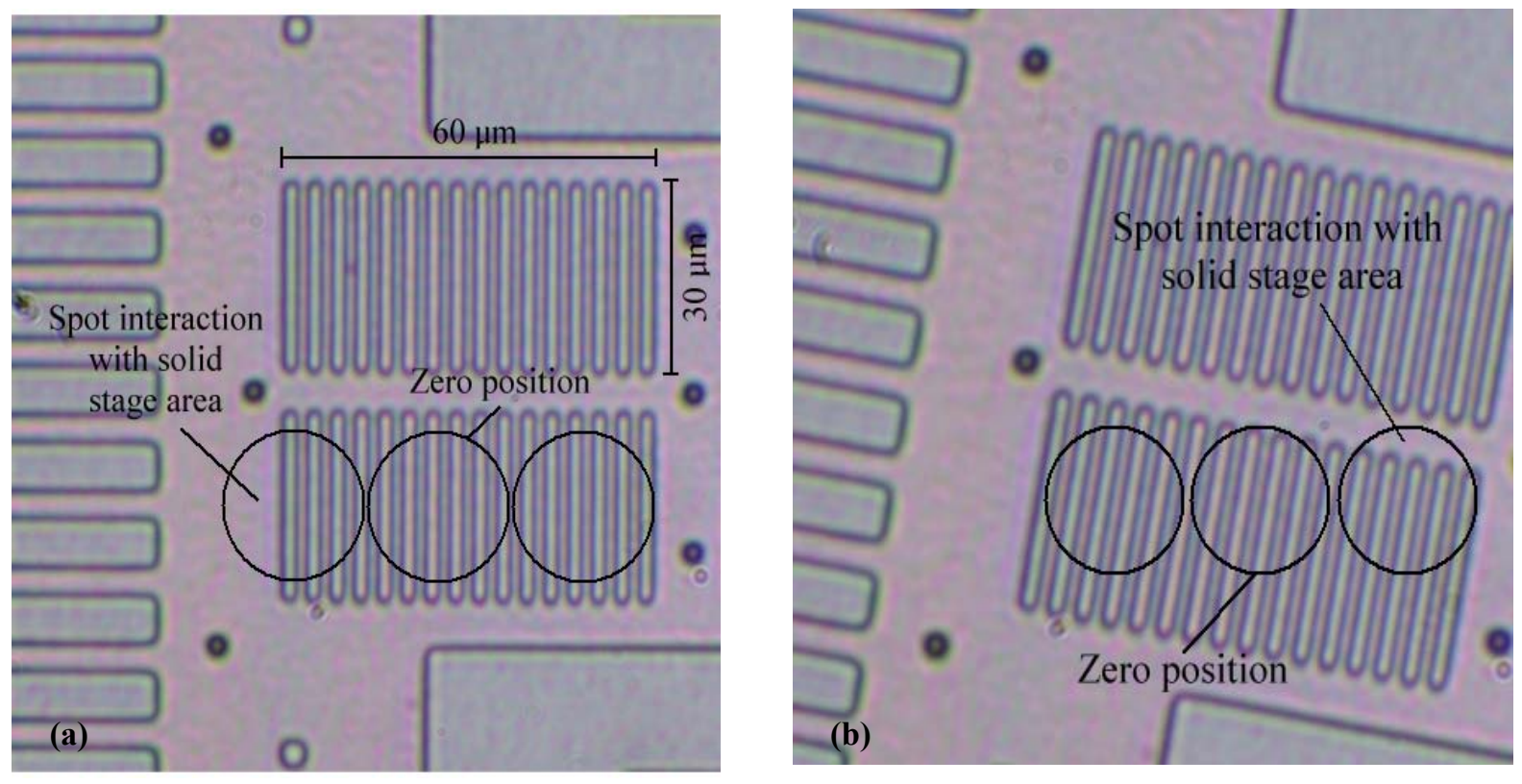

Figure 4.49: Spot interaction with solid stage areas due to large spot size and increased lateral displacement (a) and angular stage alignment (b).

This probe beam and stage interaction would have a secondary effect similar to the single opening probing area, causing one intensity change over the entire range of motion of the device, similar to the behavior present in the experimental grating optical output data. 


\section{Chapter 5 Diffractive Optical Plane Layout and Integrated Fresnel Lens Design, Fabrication, and Testing}

Chapter 5 describes the process of integrated binary Fresnel zone plate lens design and fabrication, as well as the results of their use in the through-wafer optical monitoring setup. The procedure used to generate the lens masks for the quartz waveguide substrate is described, followed by photolithography and fabrication process refinement. The final part of this chapter will present qualitative lens analysis results using visible light, and reflective path MEMS monitoring results using the waveguide substrate to deliver the probe beam.

\subsection{Lens Mask Generation}

The Fresnel diffraction theory presented in Chapter 2 was used in a C-language program that calculated the radii for the zones of the lens based on user inputs of lens size, focal length, and angle of focus. The program writes the coordinates of the calculated radii to a Caltech Intermediate Format (.cif) file that can later be converted to GDSII standard format using semiconductor design software such as L-Edit. Both on and off-axis lenses were included in the design for focus characterization purposes. Table 5.1 shows the different types of lenses included on the lens mask and the smallest zone width for each lens. The lenses are labeled on the mask according to focal distance in millimeters, length of one side of the lens square in millimeters, and angle of focus in degrees respectively. 
Table 5.1: Lens types and the smallest feature size on each lens.

\begin{tabular}{|c||c||}
\hline Layout Label & $\begin{array}{c}\text { Smallest Feature } \\
(\boldsymbol{\mu} \mathbf{m})\end{array}$ \\
\hline $5-1-0$ & 4.85 \\
\hline $5-2-0$ & 2.48 \\
\hline $5-2-10$ & 1.7 \\
\hline $5-2-15$ & 1.45 \\
\hline $5-2-20$ & 1.28 \\
\hline $5-3-0$ & 1.72 \\
\hline $5-3-10$ & 1.35 \\
\hline $5-3-20$ & 1.12 \\
\hline $10-1-0$ & 9.75 \\
\hline $10-2-0$ & 4.82 \\
\hline $10-2-10$ & 2.34 \\
\hline $10-2-15$ & 1.85 \\
\hline $10-2-20$ & 1.54 \\
\hline $10-3-0$ & 3.25 \\
\hline $10-3-10$ & 1.97 \\
\hline $10-3-20$ & 1.39 \\
\hline
\end{tabular}

This table shows that the smallest linewidth of any lens is $1.39 \mu \mathrm{m}$, a size well within the limits of the Suss MA6 alignment system. Two mask layout files, named 'toplenses_metal' and 'bottomlenses_metal', were submitted for fabrication to Photo-Sciences Inc. Both were fabricated as clear field masks, with e-beam written chrome lines on 4 in. square quartz. Quartz was chosen over soda lime to allow for short exposure times at $320 \mathrm{~nm} \mathrm{UV}$.

Figure 5.1 shows both top and bottom lens mask layouts, with each square being a lens. Alignment marks for top-to-bottom substrate alignment are located around the perimeter of the substrate area, as well as in the areas where the corners of the opposite substrate's lenses will be after final fabrication. Figure 5.1 (c) indicates the four different optical path lengths designed for coupling between input and output lenses. With a substrate thickness of 3.048 $\mathrm{mm}$ (0.12 in.), each path distance allows for 5 internal reflections at angles above the TIR requirement: $46.2^{\circ}, 51.2^{\circ}, 56.1^{\circ}$, and $61^{\circ}$. Figure 5.2 shows how lens geometry changes with changing focal length and off-axis focal angle. This figure is a good illustration of how the center point of the lens shifts as the off-axis focus angle increases. 


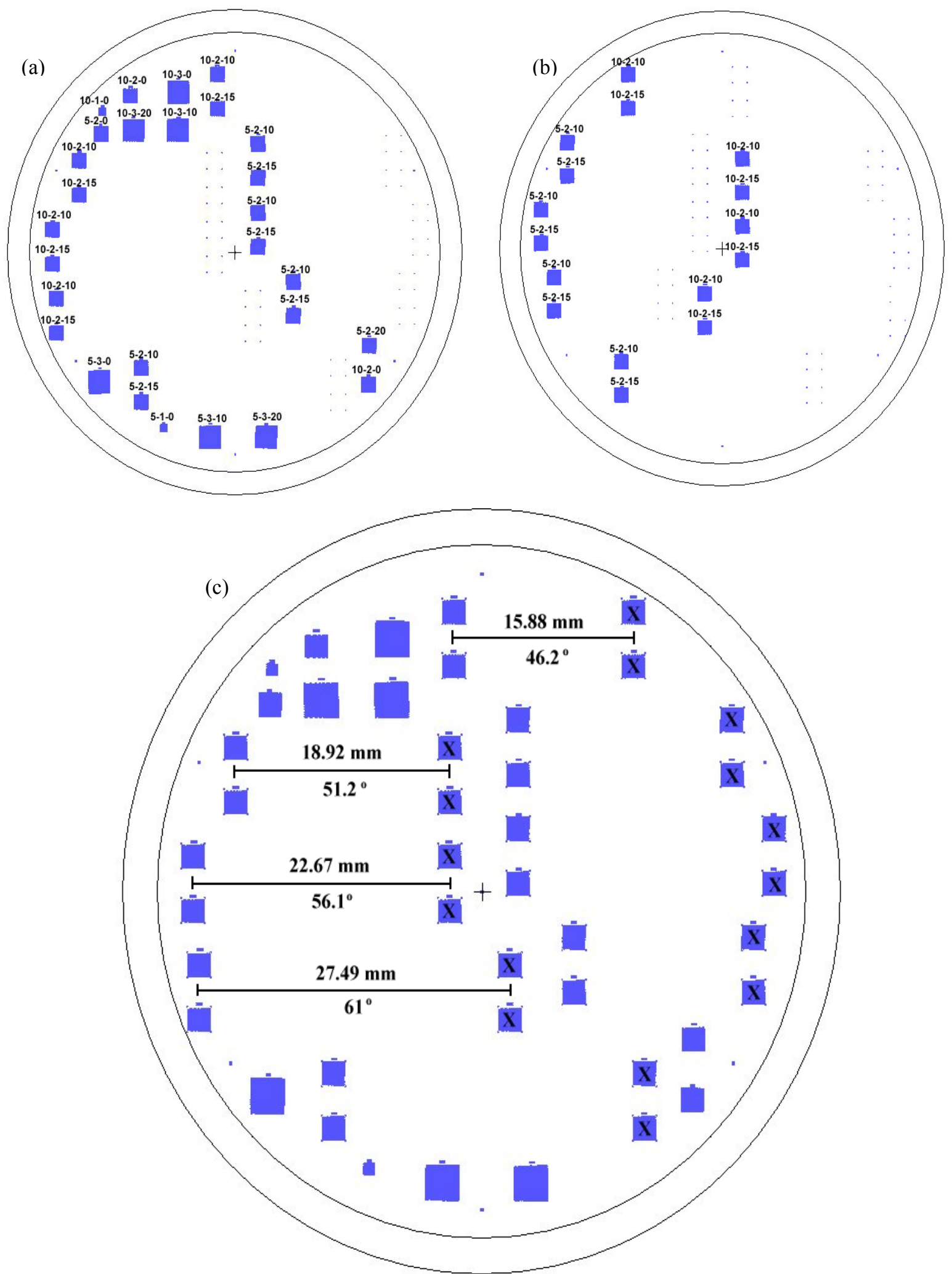

Figure 5.1: Lens mask layout: (a) top lens mask, (b) bottom lens mask, (c) both masks superimposed. Bottom lenses are denoted with an ' $x$ '. Propagation lengths indicated are for 5 internal reflections at the given angle. 


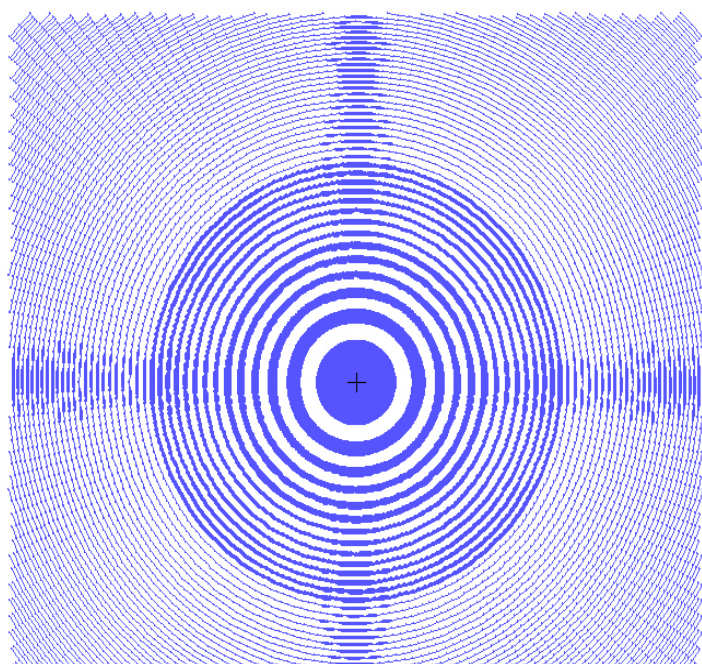

(a)

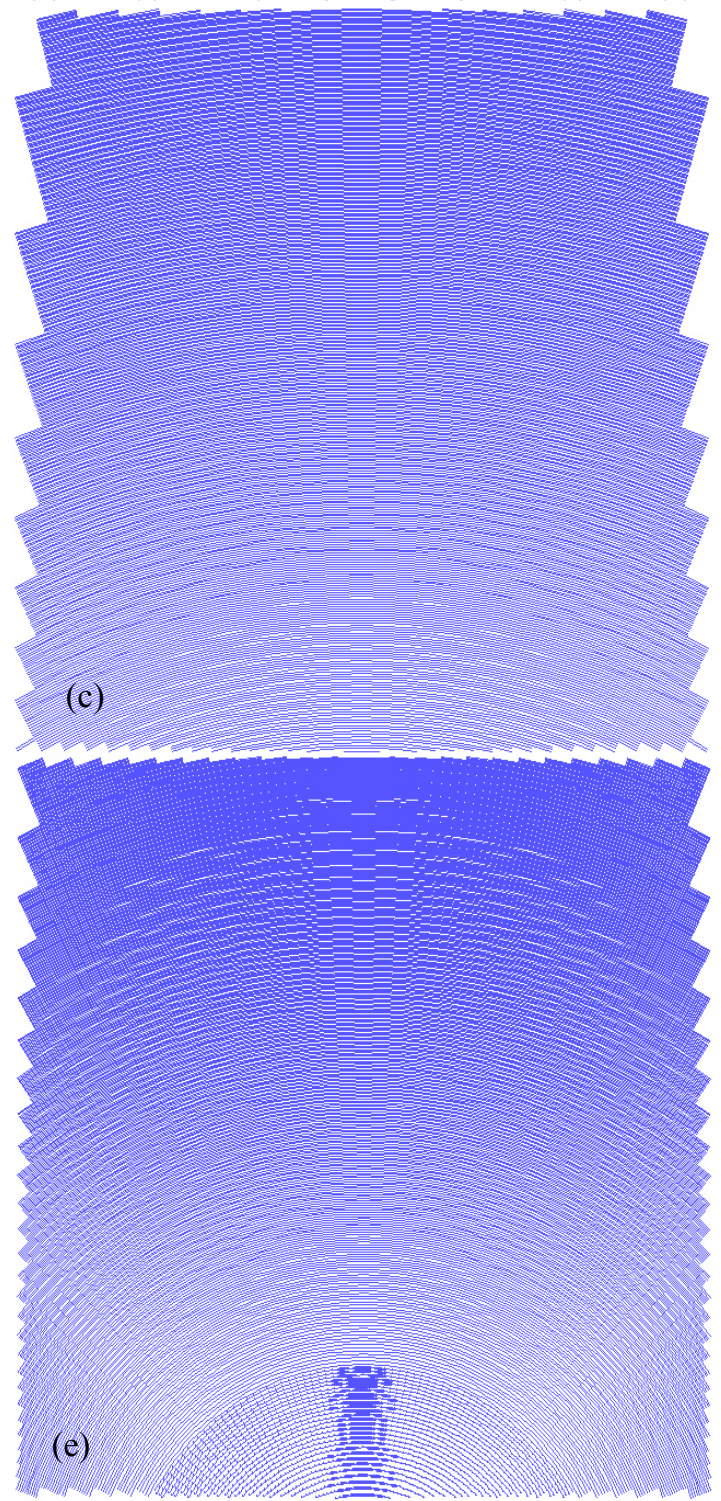

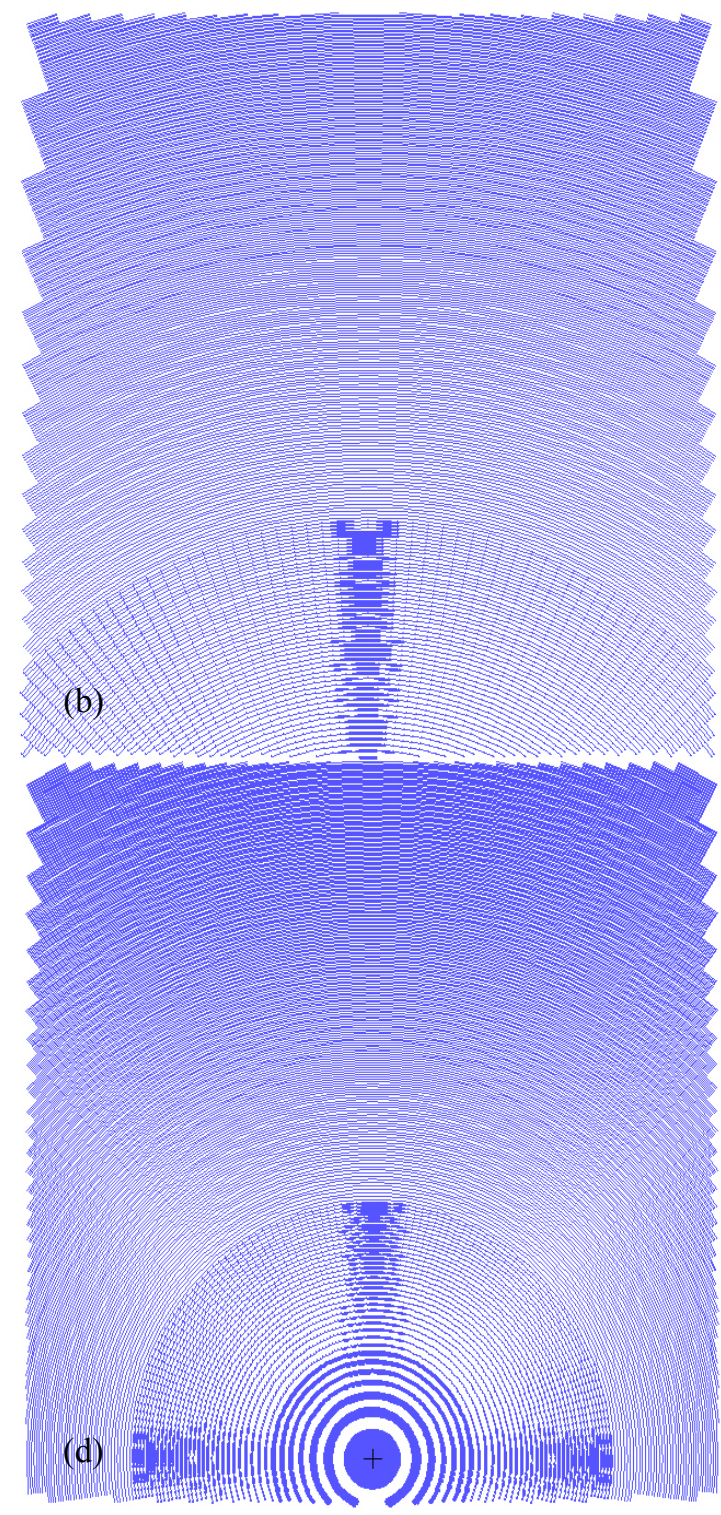

Figure 5.2: Sample lens geometries: (a) 102-0, (b) $10-2-10$, (c) $10-2-15$, (d) $5-2-10$, (e) 5-2-15. Line roughness and secondary diffraction patterns are a result of the zoom level of the layout software. 


\section{Off-Axis Device Probing}

Due to the angle of the probe beam resulting from the off-axis focusing properties of the proposed integrated optical monitoring system, the detector may experience noise caused by adjacent multiple beam intensities due to internal reflections in the oxide air gap, as illustrated in Figure 5.3.

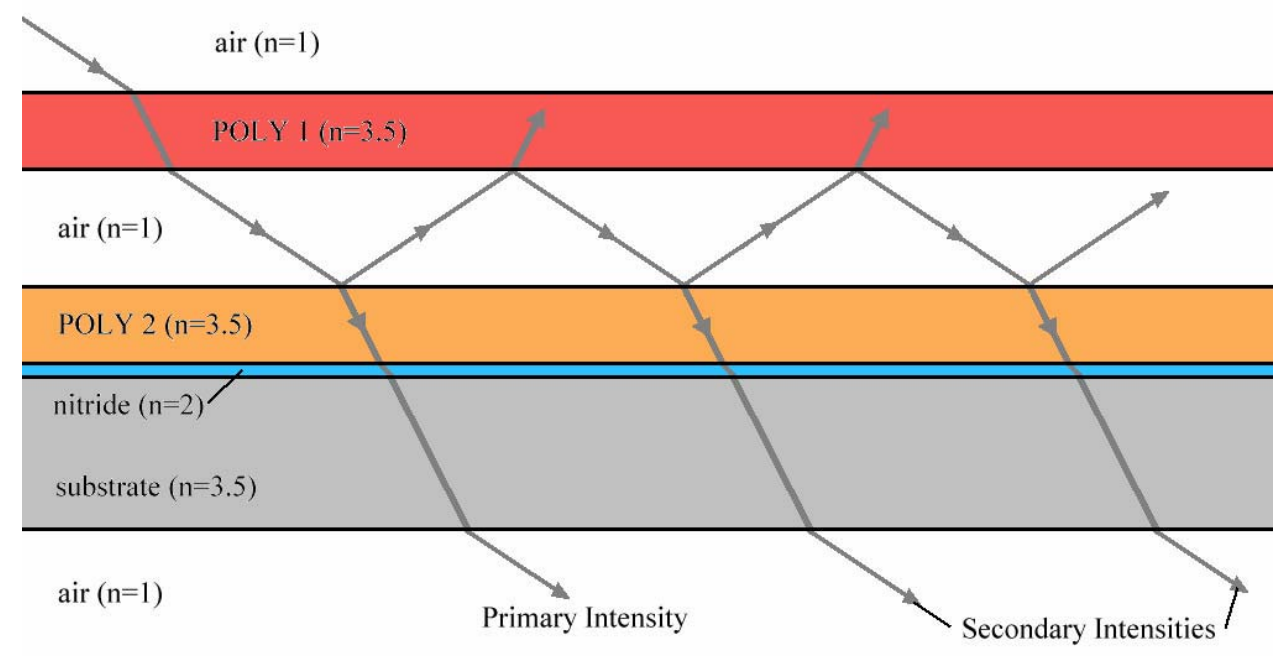

Figure 5.3: Secondary intensities caused by internal reflections in the air gap.

The relative intensity value of these secondary output intensities relative to the primary output beam intensity can be calculated using Equations 2.1-21 and 2.1-22 through 24 to find the resulting reflected and transmitted intensity values at each interface in the multilayer interaction pictured. All surfaces were assumed to be perfectly smooth in this case. Actual surface roughness will result in lower intensity values. Intensity comparison results for input angles of 0 through 90 degrees are shown in Figure 5.4. 


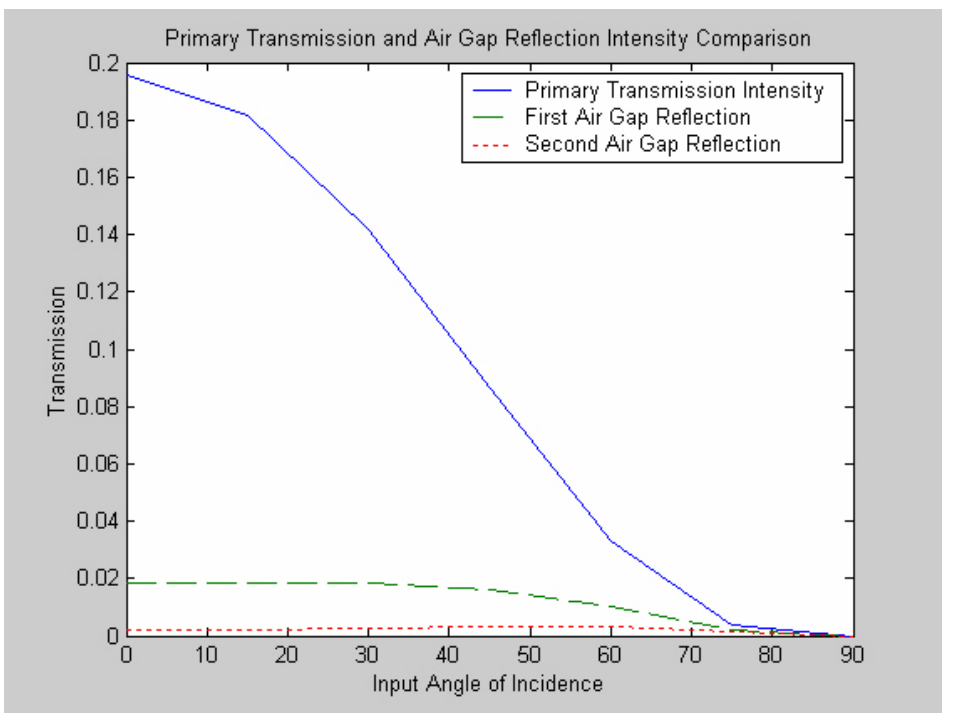

Figure 5.4: Comparison of primary off-axis probe intensity and secondary intensities caused by oxide gap reflections.

At input angles of less than 60 degrees the intensity value of the primary probe intensity is significantly larger than the secondary reflected intensities. At angles above 60 degrees, the relative intensity values are close in magnitude and could create noise in the off-axis output signal.

Figure 5.5 gives the separation distance (in microns) of the intensities for input angles of 15, 30, 45, 60, and 75 degrees. An oxide gap variation of $0.5 \mu \mathrm{m}$ has been added to determine the effects of vertical stage motion on separation distance.

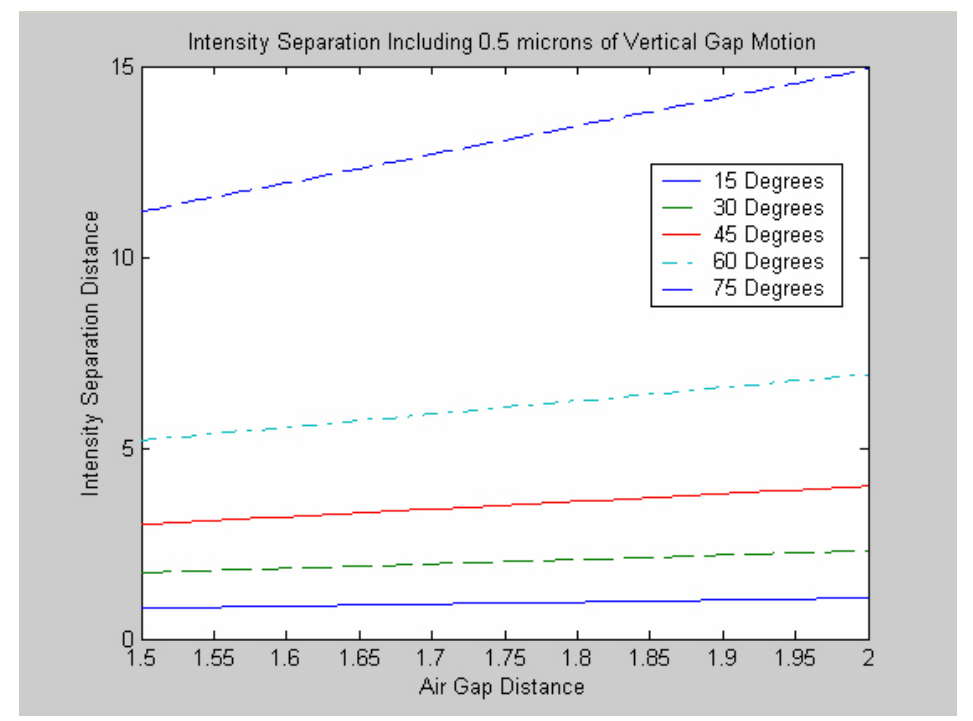

Figure 5.5: Intensity separation distance for various input angles. Separation distance change for a gap variation of 0.5 microns is included 
Intensity separation is greatest at $15 \mu \mathrm{m}$ with an input angle of 75 degrees and decreases with decreasing input angle to $\sim 1 \mu \mathrm{m}$ at 15 degrees. Maximum separation distance change over $0.5 \mu \mathrm{m}$ of oxide gap variation is $4 \mu \mathrm{m}$ for $75^{\circ}$ input. For angles below 60 degrees, the proximity of the intensities will be insignificant due to relative intensity values of the secondary intensities. No matter the input angle, output signal noise will be a concern for large spot sizes that may result in overlapping output intensity patterns.

\subsection{Photolithography}

After the masks were fabricated, characterization of the photolithograpy process was started. This was performed on 3 and 4 inch oxide-coated silicon wafers, bare silicon, and 2.5 inch quartz to determine the exposure type and time required for the small features of the lens mask. Initial studies were done using AZ5214 photoresist, a positive photoresist that has image reversal capabilities. When spun on at 5000 RPM, this photoresist is $1.4 \mu \mathrm{m}$ thick and is $\sim 1.7 \mu \mathrm{m}$ thick at 3000 RPM. The solution used to develop AZ5200 series of photoresists is AZ312 MF developer.

Image reversal was performed due to an oversight in the mask design. The clear field pattern for the lens areas is correct, but leaves the rest of the substrate bare after exposure and development, causing it to be susceptible to the etch process. A more desirable mask design would be square clear field lens patterns surrounded with metal to prevent etching of the waveguiding substrate. Due to the costly nature (because of the lengthy e-beam write time) of redesigning the masks, image reversal photoresist was chosen as a more cost effective solution, with no adverse effects on the final lens pattern. After the first exposure, the substrate is baked for 45 seconds and placed under flood exposure for 45-60 seconds to reverse the image.

The Suss MA6 aligner allowed for soft contact, hard contact, and vacuum contact exposure. For both soft and hard contact, partial pattern development occurred for lenses in the center area of the mask for all exposure times tried (ranging from 18-22 s). Longer exposure times led to overexposure of the small radius rings with no improvement on the partially developed areas. An example of this is illustrated in Figure 5.6. 


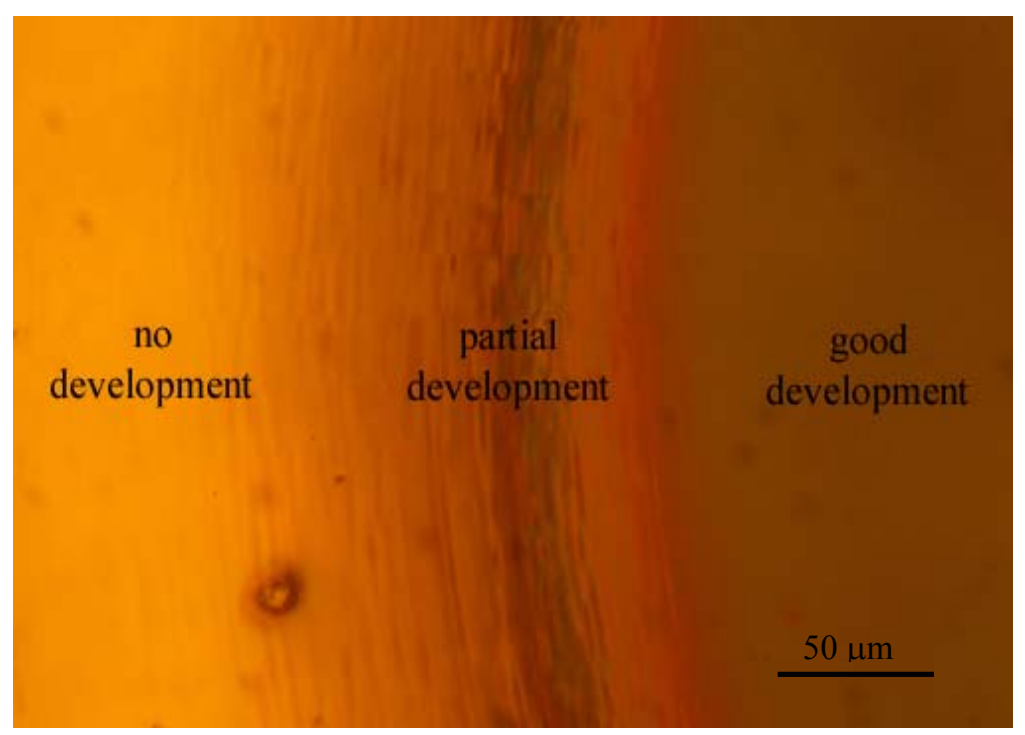

Figure 5.6: Illustration of partial pattern development due to exposure type.

This problem was solved by using vacuum contact exposure and performing photoresist lip removal. Due to the viscosity of the photoresist, a bead of photoresist exists on the edge of the substrate after spinning. The thickness of the bead prevents the substrate from coming into even contact with the mask during vacuum contact exposure. Removal of the bead was performed using a cotton swab and acetone prior to the 1 minute pre-exposure bake. Results from vacuum contact with PR lip removal were considerably better, as illustrated in Figure 5.7. Again, exposure times ranged from 18-22 seconds. Development time was $45 \mathrm{~s}$ with a developer-to-de-ionized water concentration of 1:1.5. 

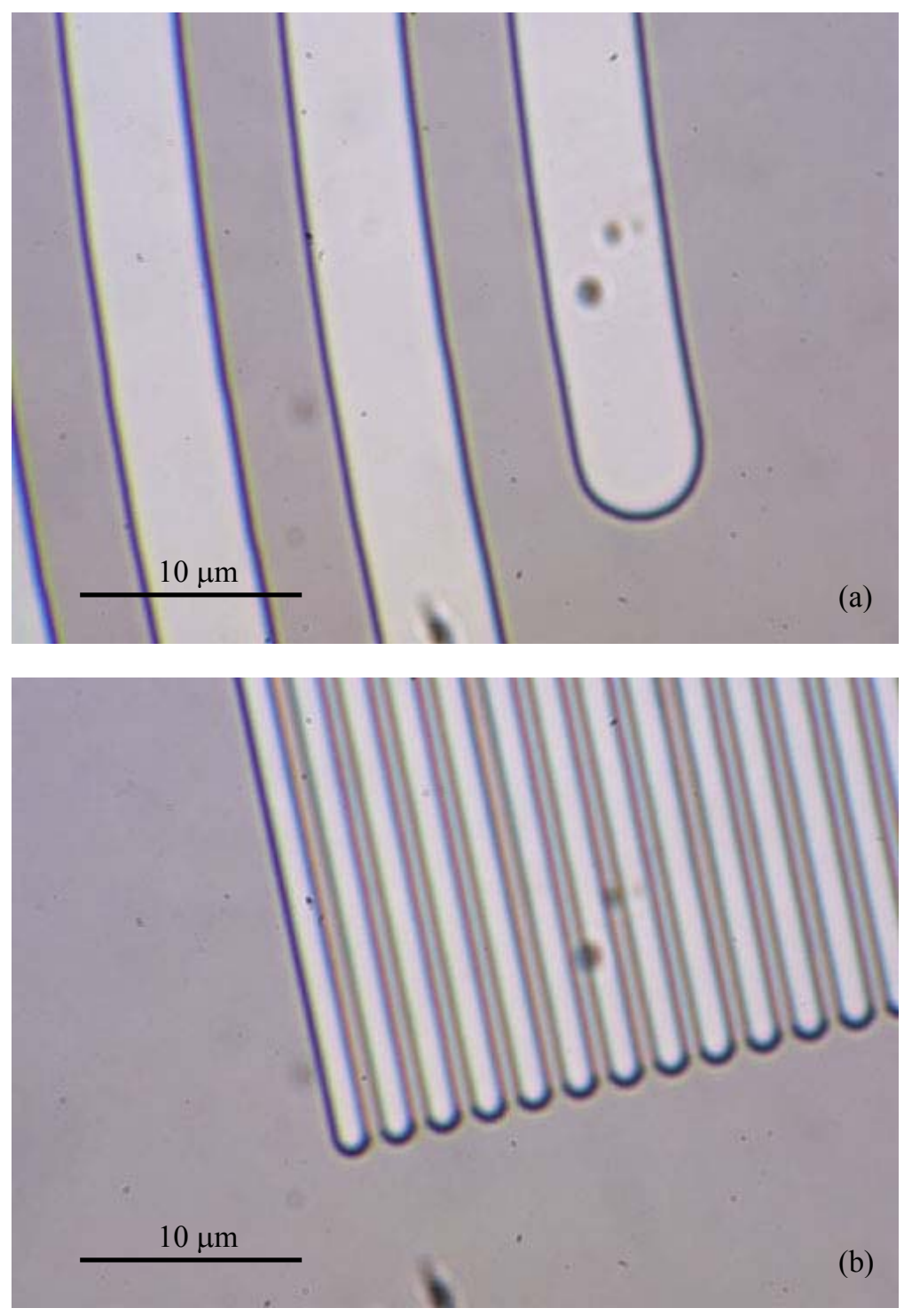

Figure 5.7: Photoresist pattern achieved with vacuum contact exposure and PR lip removal: (a) large features and (b) small lines.

Another problem occurred after successful resolution of small linewidths was acheived, photoresist de-adhesion. Because of the relatively large spacing between un-bonded atoms of the polymer photoresist at the PR-to-substrate, small lines of photoresist are less likely to adhere to the substrate surface. This is illustrated in Figure 5.8. 


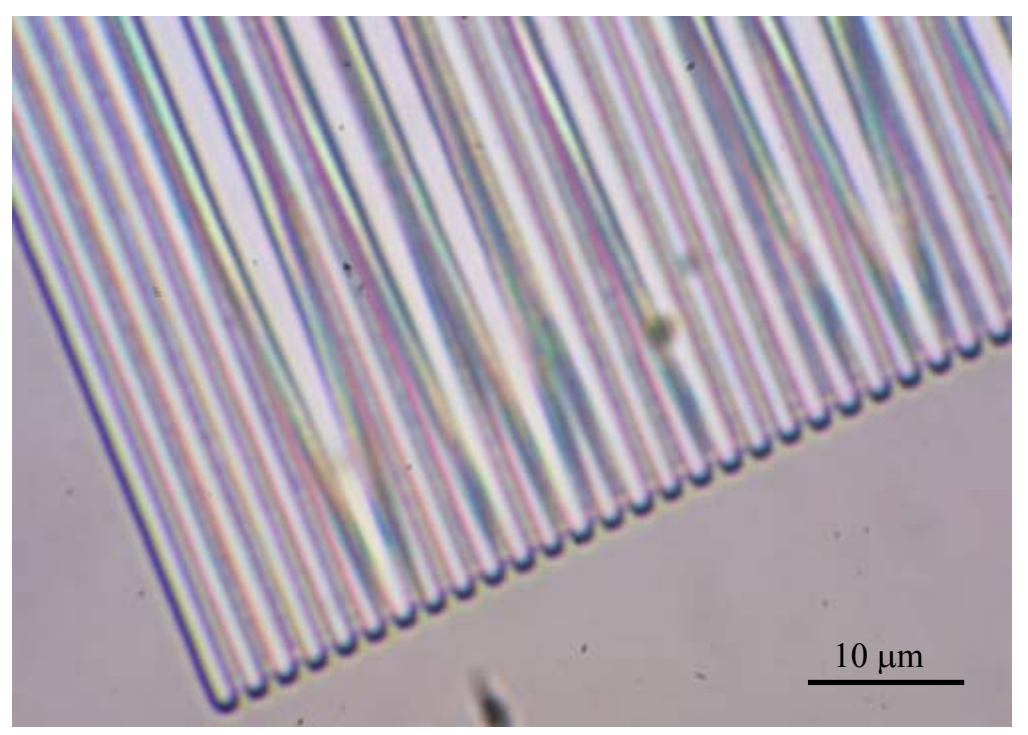

Figure 5.8: Photoresist de-adhesion.

This problem was corrected by treating the substrates with hexamethyldisilazane (HMDS), a photoresist adhesion promoter, prior to processing. This greatly improved results, but deadhesion would still occur if development times were too long.

Once the mask pattern was successfully transferred to the test substrates, the same parameters were used to begin processing the 3 inch diameter, 0.12 inch thick quartz substrates that would be used for the final product. The substrates had a surface planarity of $\pm 0.005 \mu \mathrm{m}$ and a surface quality of $40 / 20 \mathrm{scratch} / \mathrm{dig}$. Due to the reflectivity of the quartz, exposure times were increased to $28 \mathrm{~s}$ in order to fully develop the larger radii (small linewidth) rings of the off-axis lenses. Shorter exposure times led to linewidths that were too small due to the image reversal technique used, as shown in Figure 5.9. 


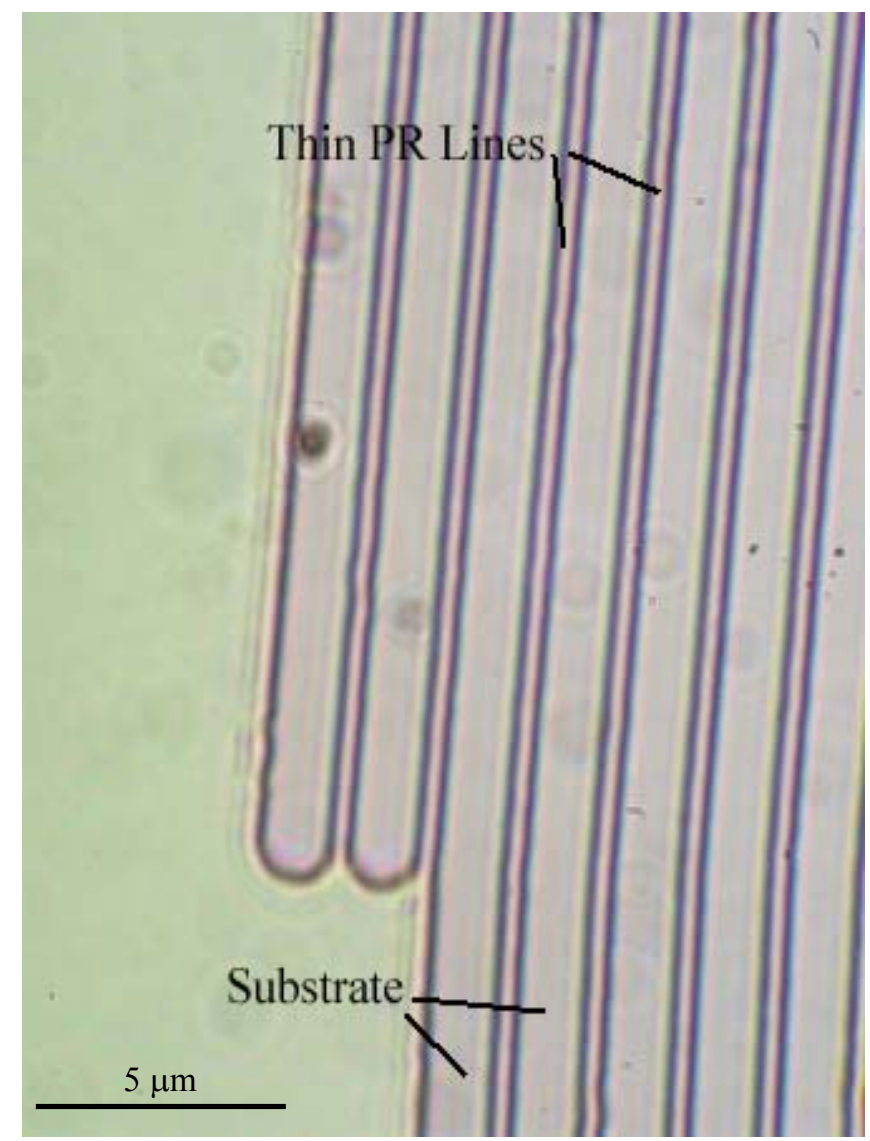

Figure 5.9: Thin photoresist lines due to underexposure.

Development time remained at 35-40 seconds, leaving the pattern slightly underdeveloped to prevent periodic PR de-adhesion. Residual photoresist would be removed by performing a short oxygen plasma descum before etching the substrate.

The type of photoresist used was changed as well. The vacuum chuck used to hold the substrates while spinning did not have enough surface area to securely hold the heavy quartz substrates in place when spun at speeds over 3500 RPM. This slow spindle speed resulted in photoresist thicknesses that were larger than the smallest linewidths in some of the lens patterns, leading to difficulty in achieving fine linewidth resolution. To alleviate this, AZ5206-E photoresist, a thinned version of AZ5214, was used. This photoresist gave thicknesses of $\sim 750 \mathrm{~nm}$ when spun on at $3500 \mathrm{RPM}$, well below the linewidth of any feature on the mask. Using this photoresist, the developer-to-DI concentration was changed to 1:2. Figure 5.10 shows successful photoresist patterning on 3 inch quartz using the adjusted photolithography parameters. 


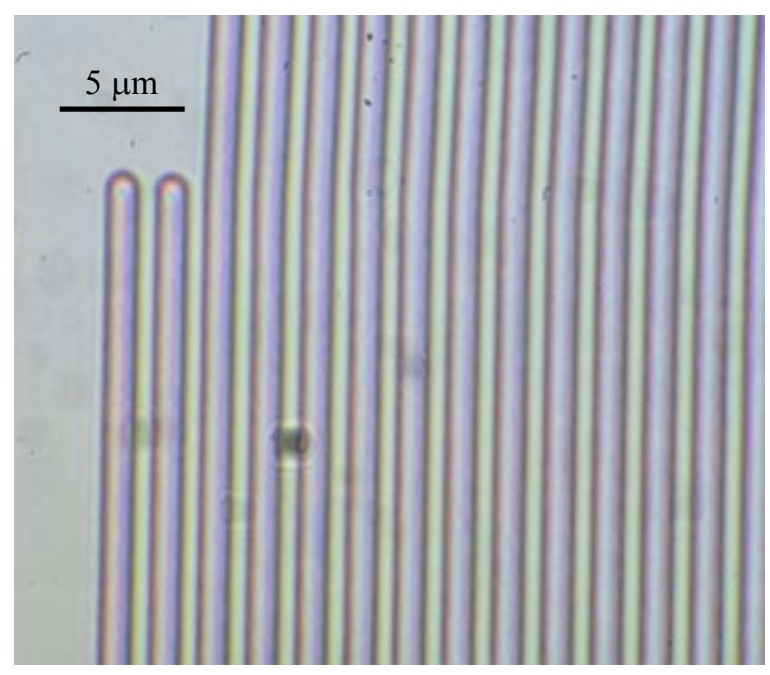

(a)

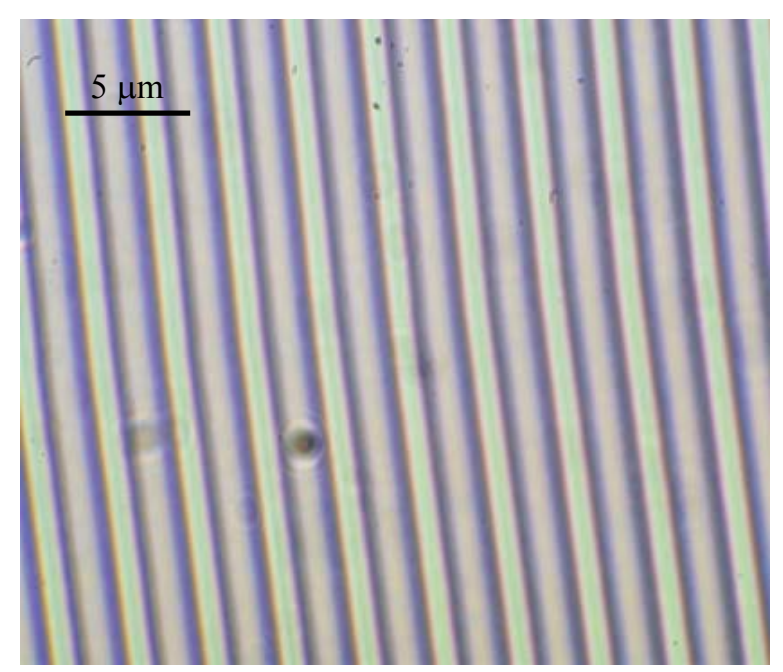

(b)

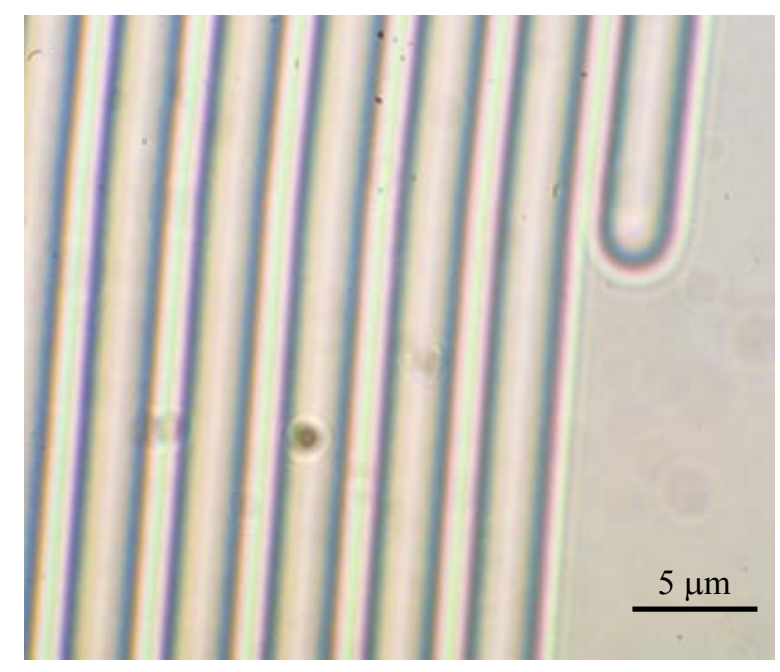

(c)

Figure 5.10: Successful photoresist patterning on 3 in. quartz: (a) small radii rings, (b) medium radii rings, and (c) large radii rings. 
Table 5.2 presents a comparison of selected mask (desired) linewidths to actual PR linewidths obtained using the optimum photolithography process described. Actual linewidths were determined using a microscope objective reticule.

Table 5.2: Comparison of desired and photoresist linewidths.

\begin{tabular}{||c|c|c|c|c||}
\hline Lens Label & $\begin{array}{c}\text { Small Feature PR } \\
\text { Linewidth }(\boldsymbol{\mu m})\end{array}$ & $\begin{array}{c}\text { Mask Linewidth } \\
(\boldsymbol{\mu} \mathbf{m})\end{array}$ & $\begin{array}{c}\text { Large Feature PR } \\
\text { Linewidth }(\boldsymbol{\mu} \mathbf{m})\end{array}$ & $\begin{array}{c}\text { Mask Linewidth } \\
(\boldsymbol{\mu} \mathbf{m})\end{array}$ \\
\hline \hline $5-2-10$ & 1.9 & 1.8 & 32 & 33.5 \\
\hline $5-2-15$ & 1.4 & 1.5 & 9.8 & 9.4 \\
\hline $10-2-10$ & 2.3 & 2.4 & 9.2 & 8.6 \\
\hline $10-2-15$ & 1.6 & 1.9 & 8 & 5 \\
\hline $10-3-20$ & 1.2 & 1.4 & 2.8 & 3.1 \\
\hline $5-3-20$ & 0.9 & 1.1 & 10.3 & 9.9 \\
\hline
\end{tabular}

This data shows an average small linewidth variation of $0.2 \mu \mathrm{m}$ and an average large linewidth variation of $0.66 \mu \mathrm{m}$, within the range of acceptable transferred linewidth error.

\subsection{Reactive Ion Etch}

After a repeatable photolithography process was developed, etch studies were begun. Before patterning substrates for etching, the photomasks were cleaned in a room temperature ultrasonic methanol bath for 20 minutes to remove photoresist residue present between the metal mask lines as a result of repetitive processing. Because of the limited number of quartz substrates available, initial etch processes were first performed on patterned oxide coated 4 inch silicon wafers due to its similarities to quartz. Table 5.3 outlines the parameters for the $\mathrm{SiO}_{2}$ Fluoroform etch process and corresponding etch depths. The substrate etch was preceded by a $30 \mathrm{~s}$ Oxygen descum at $300 \mathrm{mT}$ with a gas flow of $25 \mathrm{sccm}$ and $150 \mathrm{~W}$ of RF power. The etch itself was performed at the parameters in Table 5.3 with a gas flow of 110 sccm, followed by a 10-12 min Oxygen resist strip at $300 \mathrm{mT}, 30 \mathrm{sccm}$, and $200 \mathrm{~W}$ of RF power. All quartz etching was performed at $110 \mathrm{sccm}$ because the mass flow controller could not stabilize gas flows below this level, causing RF power failure and abortion of the process. 
Table 5.3: Silicon oxide etch test results.

\begin{tabular}{|c|c|c|c|c|}
\hline \multicolumn{5}{|c|}{ Change Process Pressure } \\
\hline \hline Time $(\mathrm{m})$ & Process Pressure $(\mathrm{mT})$ & RF Power $(\mathrm{W})$ & Etch Depth $(\mathrm{kA})$ & Text "0" Linewidth (microns) \\
\hline 10 & 30 & 150 & 1.48 & 20.4 \\
\hline 10 & 60 & 150 & 1.07 & 24 \\
\hline
\end{tabular}

\begin{tabular}{|c|c|c|c|c|}
\hline \multicolumn{5}{|c|}{ Change Process Time } \\
\hline \hline Time $(\mathrm{m})$ & Process Pressure $(\mathrm{mT})$ & RF Power $(\mathrm{W})$ & Etch Depth $(\mathrm{kA})$ & Text "0" Linewidth (microns) \\
\hline 5 & 30 & 150 & 0.81 & 22 \\
\hline 10 & 30 & 150 & 1.48 & 20.4 \\
\hline 15 & 30 & 150 & 2.72 & 20 \\
\hline
\end{tabular}

\begin{tabular}{|c|c|c|c|c|}
\hline \multicolumn{5}{|c|}{ Change Process Power } \\
\hline \hline Time $(\mathrm{m})$ & Process Pressure $(\mathrm{mT})$ & RF Power $(\mathrm{W})$ & Etch Depth $(\mathrm{kA})$ & Text "0" Linewidth (microns) \\
\hline \hline 10 & 30 & 100 & 1.01 & 21.6 \\
\hline 10 & 30 & 150 & 1.48 & 20.4 \\
\hline 10 & 30 & 200 & 1.86 & 21.6 \\
\hline
\end{tabular}

It can be seen in this data that increasing etch time had the greatest effect in increasing etch depth. Process pressures above $30 \mathrm{mT}$ slowed etch rates. Power levels above $150 \mathrm{~W}$ caused excessive photoresist loss during the fluoroform etch, resulting in a degradation of the etched pattern. Photoresist loss at $150 \mathrm{~W}$ was measured to be $\sim 10 \mathrm{~nm}$ per minute. Desired etch depth was determined to be $1.31 \mu \mathrm{m}$ by using half of the value obtained using Equation 2.3-4, the value needed for a binary lens. This etch depth would result in etch times in excess of one hour, allowing the photoresist to be removed before the etch process was finished. To accommodate this fact, and etch depth of $436 \mathrm{~nm}\left(\lambda / 2 n_{\text {medium }}\right)$ was chosen to shorten the time to achieve the desired depth.

After determining the optimal power, gas flow, and process pressure parameters, variation in etch time was performed on both 2.5 and 3 in. quartz substrates. The results are presented in Table 5.4 . 
Table 5.4: Etch depths for quartz. All times performed with fluoroform at $30 \mathrm{mT}, 100 \mathrm{sccm}$, and $150 \mathrm{~W}$.

\begin{tabular}{|c|c|}
\hline Time (m) & Etch Depth (nm) \\
\hline \hline 15 & 180 \\
\hline 20 & 235 \\
\hline 25 & 285 \\
\hline 30 & 345 \\
\hline 35 & 395 \\
\hline 45 & 525 \\
\hline
\end{tabular}

This data indicated that an etch time of 40 minutes would result in a depth close to the desired depth of $436 \mathrm{~nm}$.

After the process time required to achieve the desired etch depth was determined, front and back side processing was performed. To protect the small etched features on the top side of the substrate during back side processing, the top surface of the substrate was coated with photoresist and processing proceeded as normal. Top-to-bottom side alignment was performed using the back side alignment feature of the MA6 mask aligner. Figure 5.11 shows the results of a reactive ion fluoroform etch at $30 \mathrm{mT}, 100 \mathrm{sccm}$, and $150 \mathrm{~W}$ for 40 minutes. 


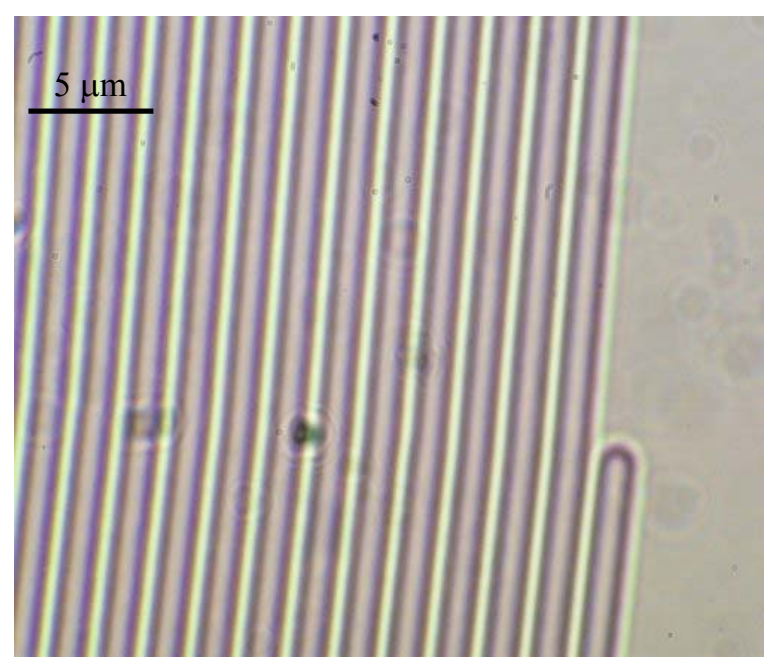

(a)

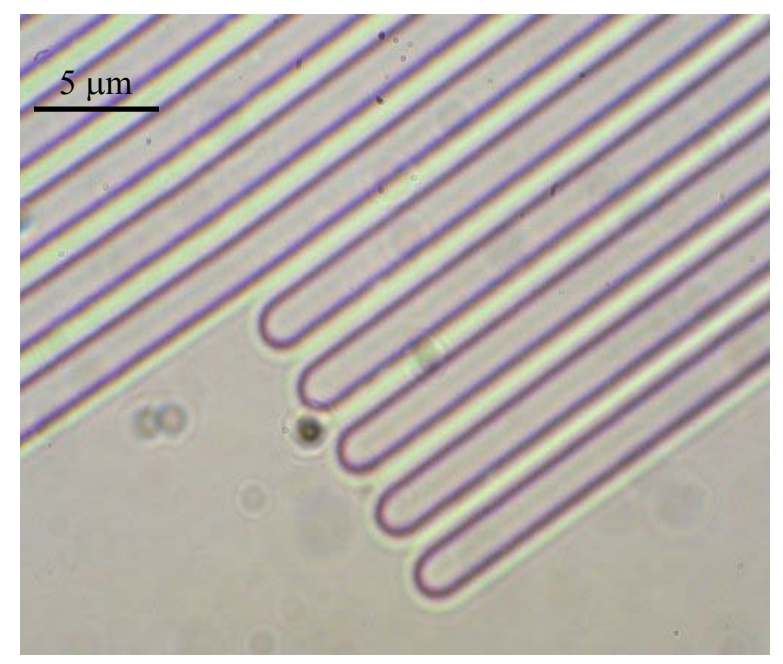

(b)

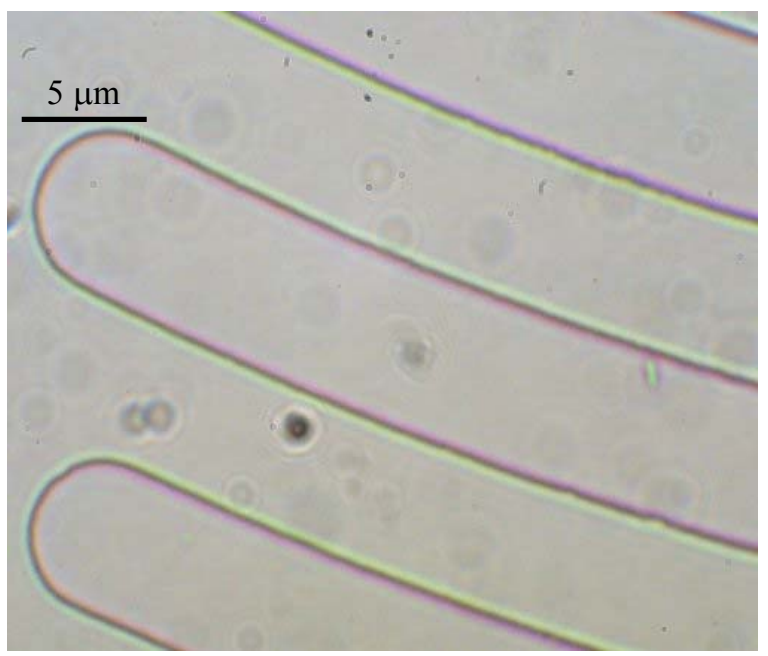

(c)

Figure 5.11: Successful etch patterning on 3 in. quartz: (a) small radii rings, (b) medium radii rings, and (c) large radii rings. 
Table 5.5 compares desired mask linewidths and actual linewidths resulting from the etch process.

Table 5.5: Comparison of desired and etched linewidths.

\begin{tabular}{||c|c|c|c|c||}
\hline \hline Lens Label & $\begin{array}{c}\text { Small Feature } \\
\text { Etched Linewidth } \\
(\boldsymbol{\mu} \mathbf{m})\end{array}$ & $\begin{array}{c}\text { Mask Linewidth } \\
(\boldsymbol{\mu} \mathbf{m})\end{array}$ & $\begin{array}{c}\text { Large Feature } \\
\text { Etched Linewidth } \\
(\boldsymbol{\mu} \mathbf{m})\end{array}$ & $\begin{array}{c}\text { Mask Linewidth } \\
(\boldsymbol{\mu} \mathbf{m})\end{array}$ \\
\hline \hline $5-2-10$ & 1.9 & 1.8 & 32 & 33.5 \\
\hline $5-2-15$ & 1.4 & 1.5 & 9.2 & 9.4 \\
\hline $10-2-10$ & 1.9 & 2.4 & 8 & 8.6 \\
\hline $10-2-15$ & 1.2 & 1.9 & 4.6 & 5 \\
\hline $10-3-20$ & 1.4 & 1.4 & 3.4 & 3.1 \\
\hline $5-3-20$ & 0.9 & 1.1 & 10.3 & 9.9 \\
\hline
\end{tabular}

This data shows an average small linewidth error of $0.26 \mu \mathrm{m}$. This error, resulting in linewidths smaller than the desire mask feature size, is most likely due to photoresist loss during the plasma etch process. Average large linewidth error is $0.56 \mu \mathrm{m}$. Figure 5.12 shows a completed substrate with top and bottom lenses, with an etch depth measured to be $\sim 450$ nm.

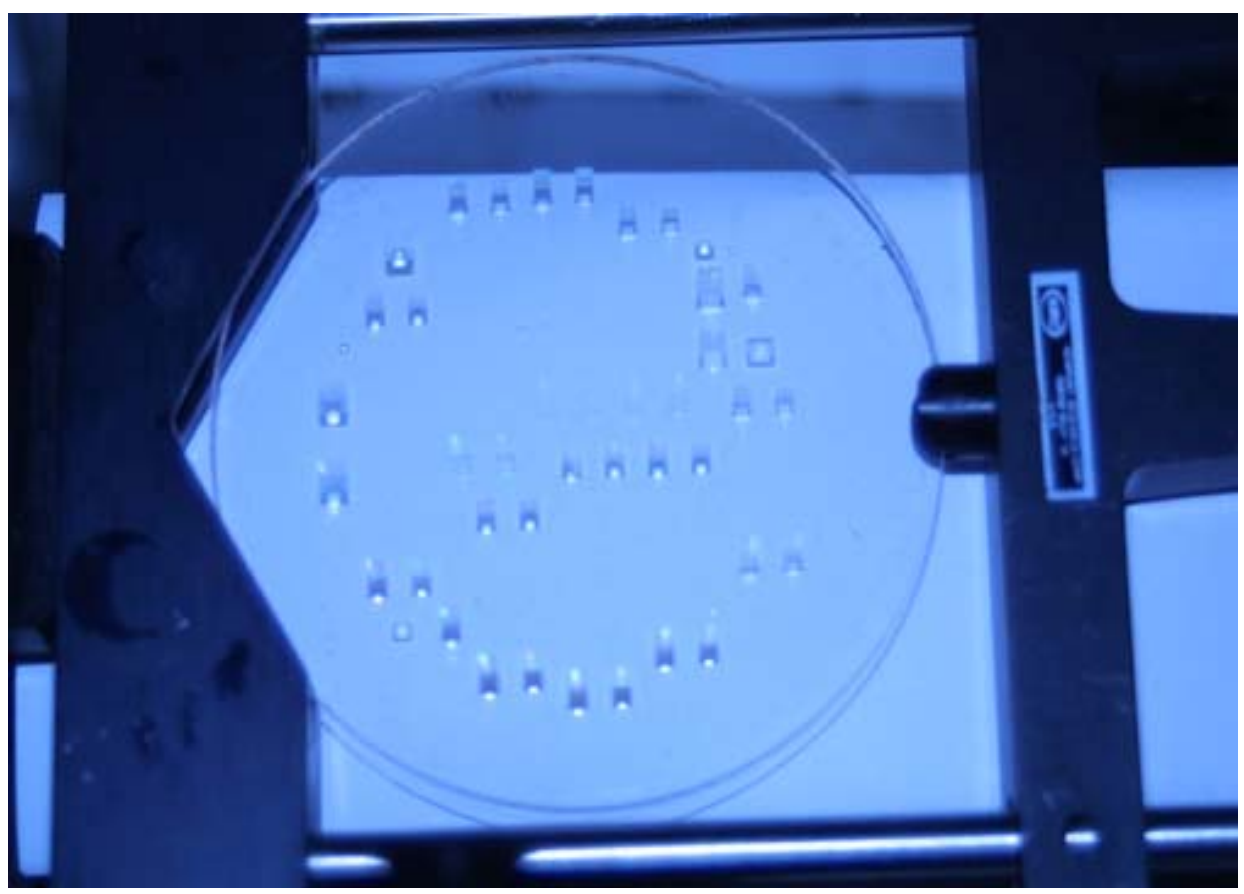

Figure 5.12: Completed lens substrate with top and bottom lenses. Spots are diffracted and focused light from the camera flash. 
The central area of the back side etched substrate pictured experienced excessive photoresist loss during etch processing, resulting in less than optimal etch depth $(\sim 250 \mathrm{~nm})$, but bottom side lenses near the edge of the substrate were close to the desired value. Spots near each lens in Figure 5.12 are diffracted and focused light form the camera flash.

Figure 5.13 outlines the fabrication process flow used to create lens substrates from photolithography to RIE processing.

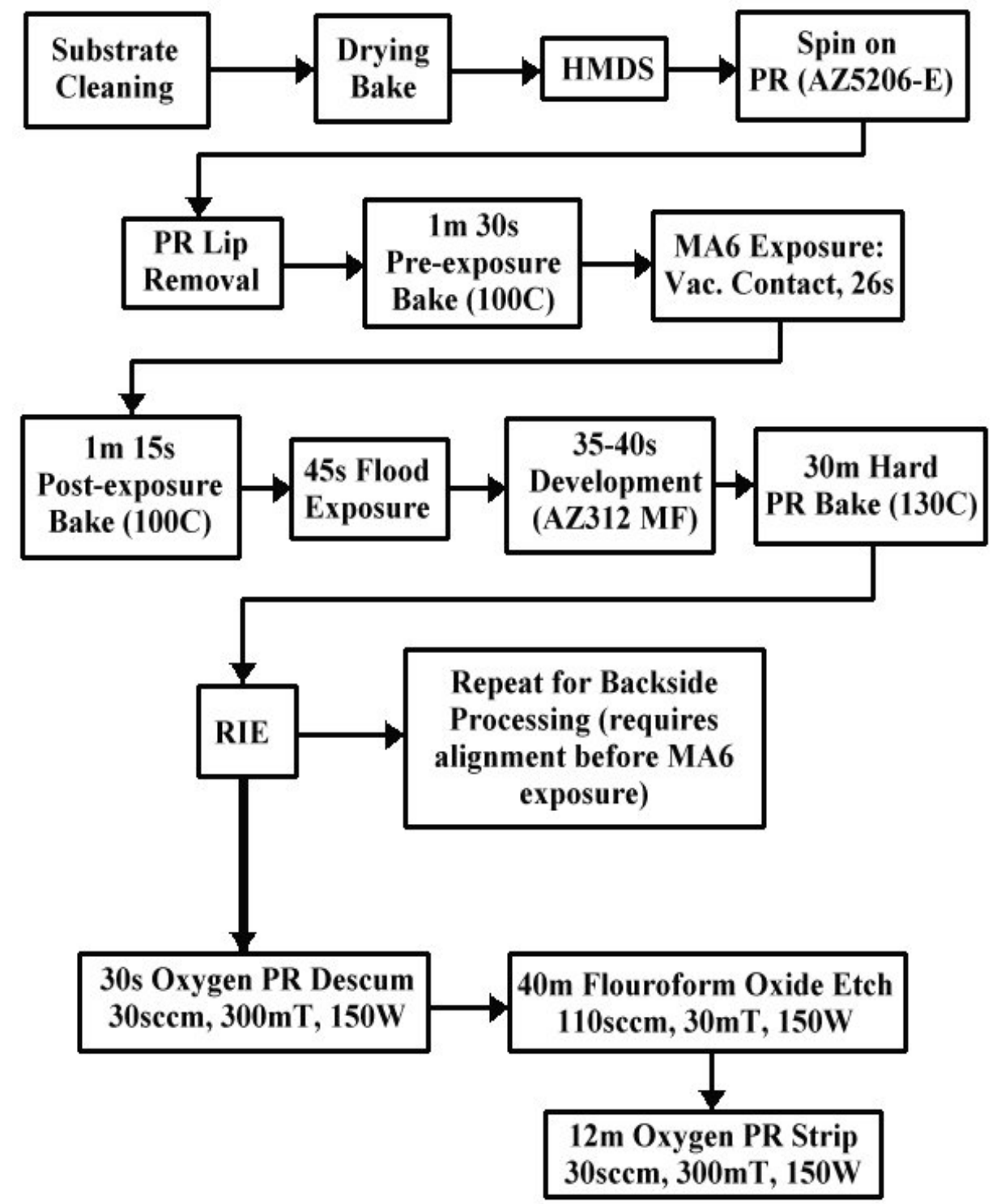

Figure 5.13: Lens substrate process flow. 


\subsection{Beam Coupling and Analysis}

Successful fabrication of Fresnel lenses on the front and back sides of the quartz waveguide substrate was followed by an analysis of the coupling and focusing abilities of the microoptical elements. This examination was performed using visible light $(630 \mathrm{HeNe})$ to aid in alignment and focal point determination. Figure 5.14 gives a schematic representation of the testing setup used.

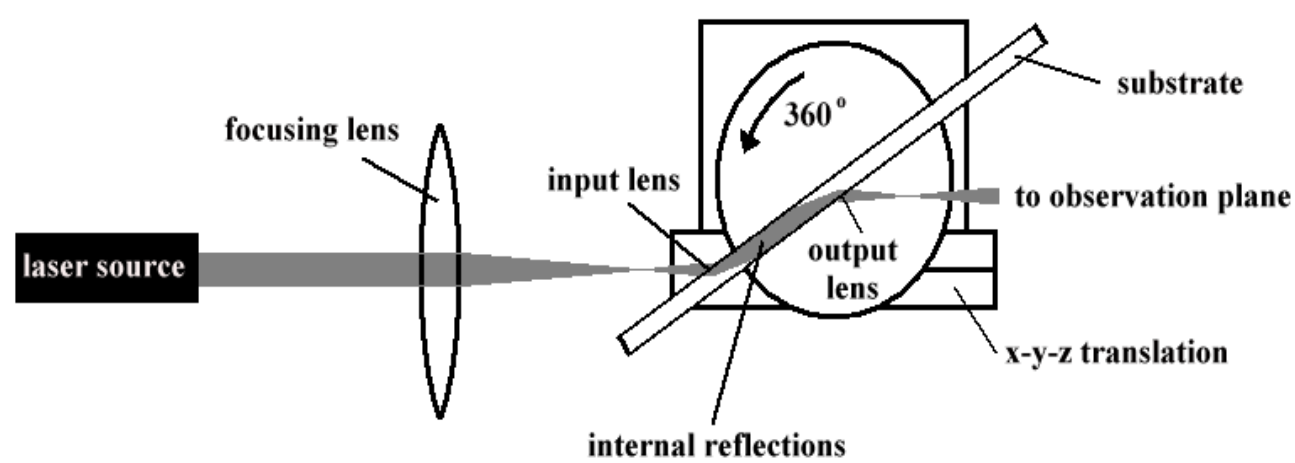

Figure 5.14: Fresnel lens analysis setup.

The lens substrate was mounted on an $x-y-z$ translation stage for directional adjustment and a 360 degree rotational stage for input beam angle adjustment. The input beam was focused by a bulk optical lens, and the position of the substrate was adjusted to put the focal point of the bulk optical lens $(40 \mathrm{~mm})$ at the location of the focal point of the integrated lens. By using the $\pi / 2$ radius equation from Table 2.1 to solve for focal length, coupling input light at 630 $\mathrm{nm}$ results in a doubling of the designed focal distance.

As a pre-etch evaluation of the diffraction effectiveness of the lens patterns, a beam was coupled into the quartz substrate using a $1.7 \mu \mathrm{m}$ thick AZ5214 PR top-lens pattern. Results are shown in Figure 5.15, with internal reflection readily visible, indicating propagation in the substrate. 


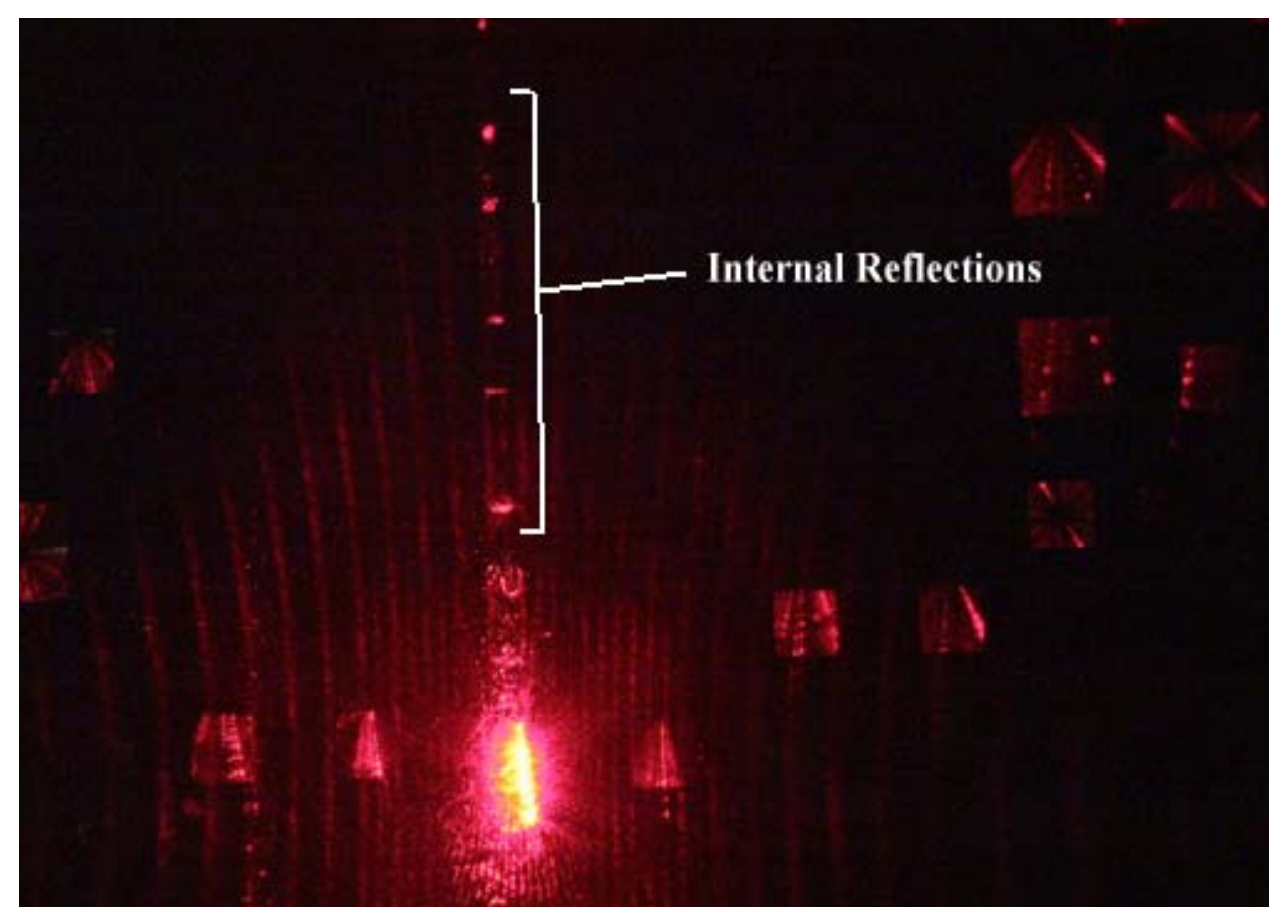

Figure 5.15: Beam coupling using an early AZ5214 photoresist pattern.

An examination of the possible effects that the etch process could have on the transmitted beam intensity was performed using a substrate that was half etched and half un-etched. Results are shown in Figure 5.16.
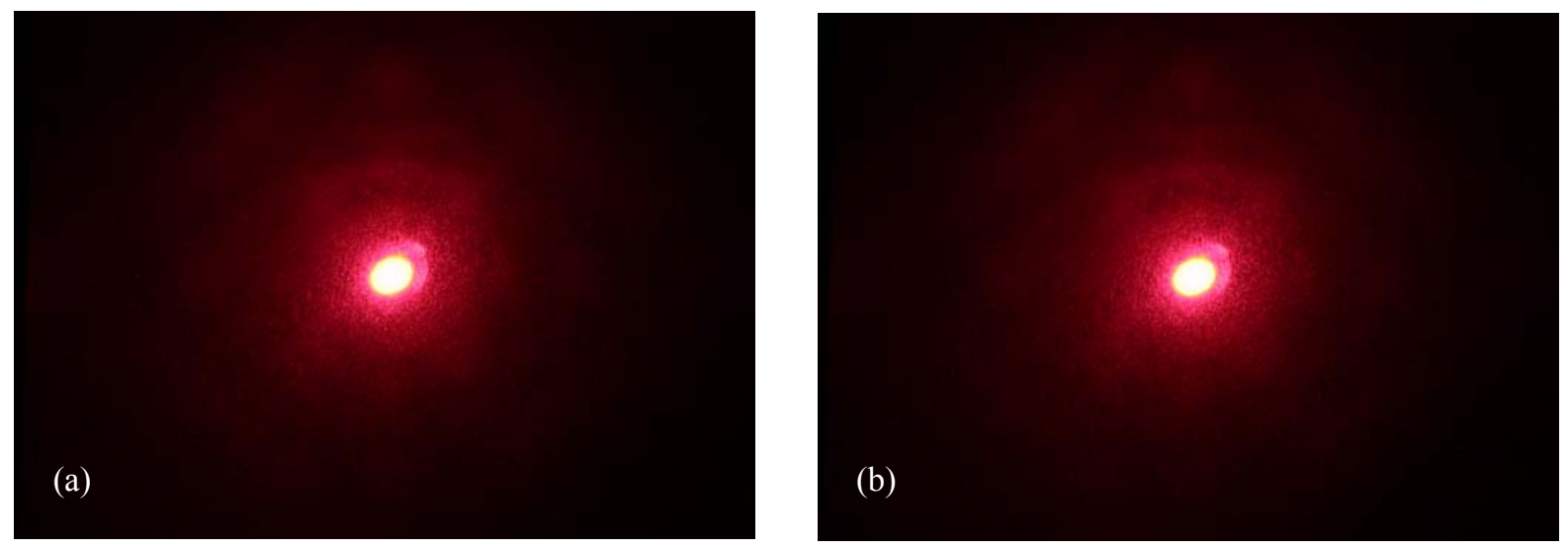

Figure 5.16: Etch effect on transmitted beam intensity: (a) un-etched substrate area and (b) etched substrate area.

It can be seen from this figure that the etch process had no appreciable effect on the output intensity, mainly because the surface variations are significantly less than the beam wavelength. 
The lens substrate pictured in Figure 5.12 was examined next to analyze the coupling and focusing properties of the etched lens patterns. On-axis lenses designed for 5 and $10 \mathrm{~mm}$ focal lengths were examined next with the bulk optical lens removed from the setup and the substrate situated 90 degrees to the incoming beam. Both behaved as predicted, focusing light at 1 and $2 \mathrm{~cm}$, respectively. Near and far-field intensity patterns for a $2 \mathrm{~cm}$ focus lens are shown in Figure 5.17.
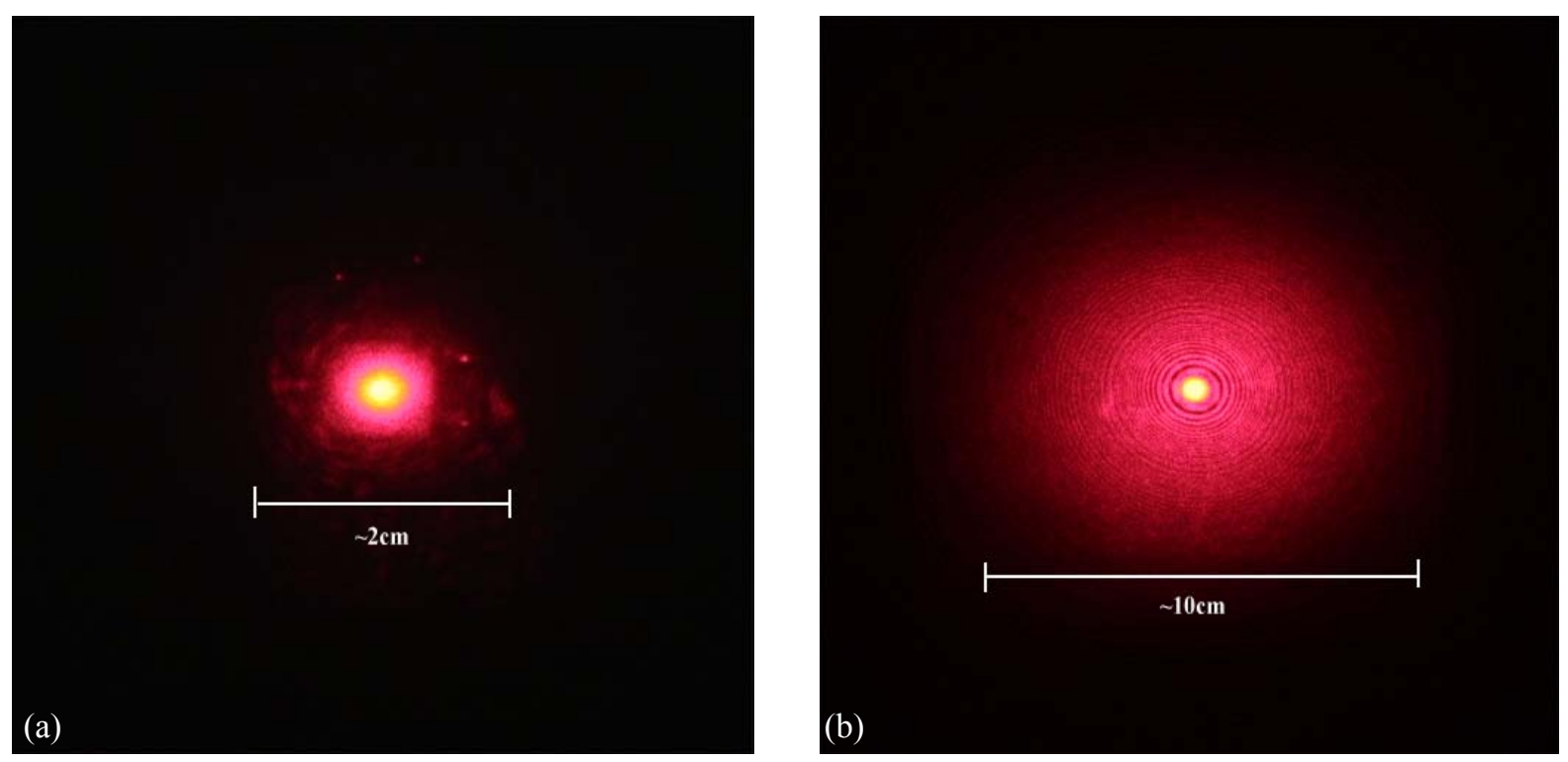

Figure 5.17: On-axis intensity patterns at (a) $2 \mathrm{~cm}$ and (b) $20 \mathrm{~cm}$.

Intensity patterns appeared circularly symmetric at both distances, with lens diffraction effects readily visible in the far field photo.

An off-axis lens with HeNe focal length of $1 \mathrm{~cm}$ and 10 degrees of off-axis focus was examined next. The lens substrate was adjusted to a 36 degree tilt to achieve the proper internal reflection angle for 5 reflections from input to output. The resulting near-field intensity pattern is shown in Figures 5.18 and 5.19. 


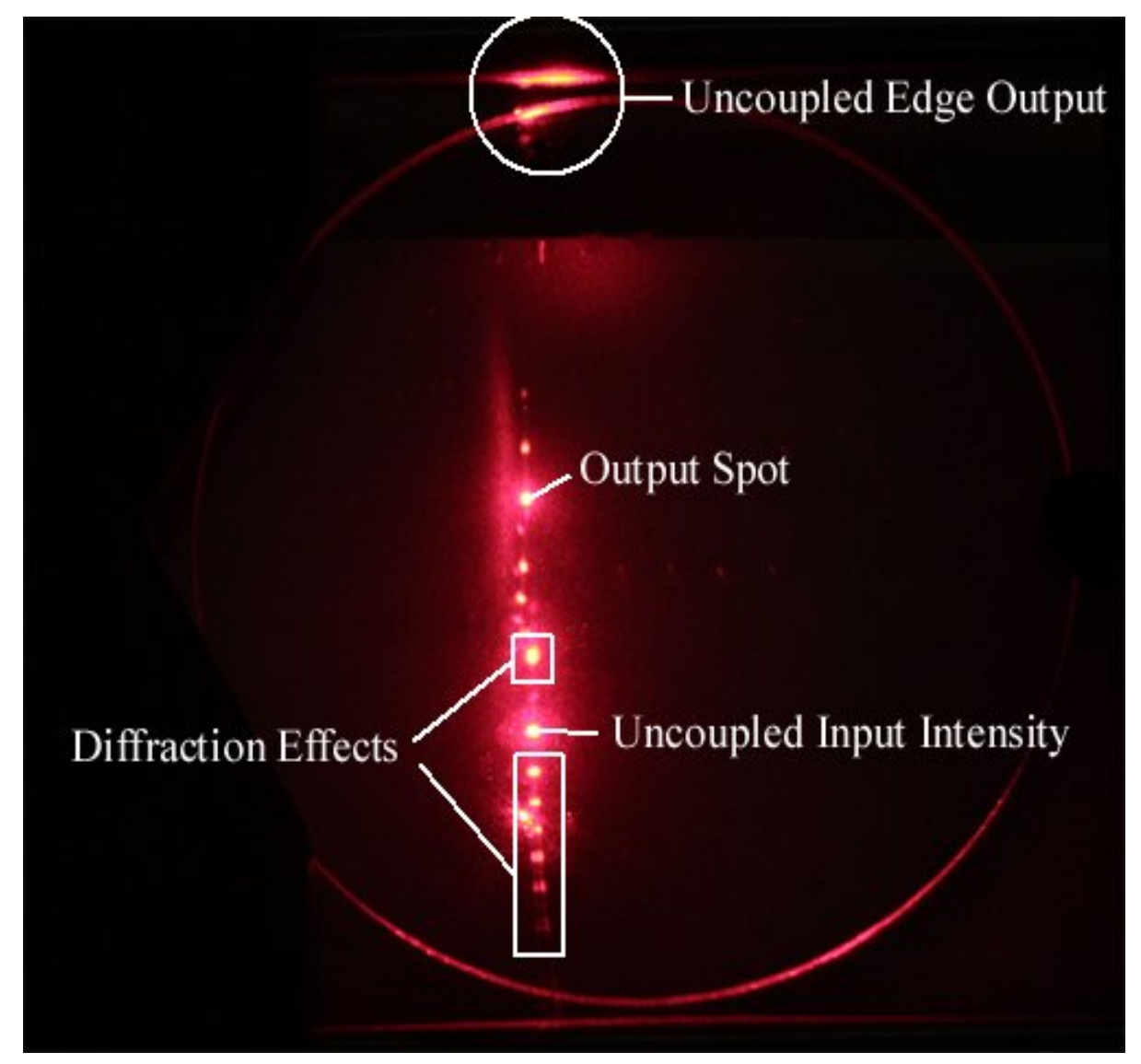

Figure 5.18: Coupled off-axis beam intensity at $1 \mathrm{~cm}$. 


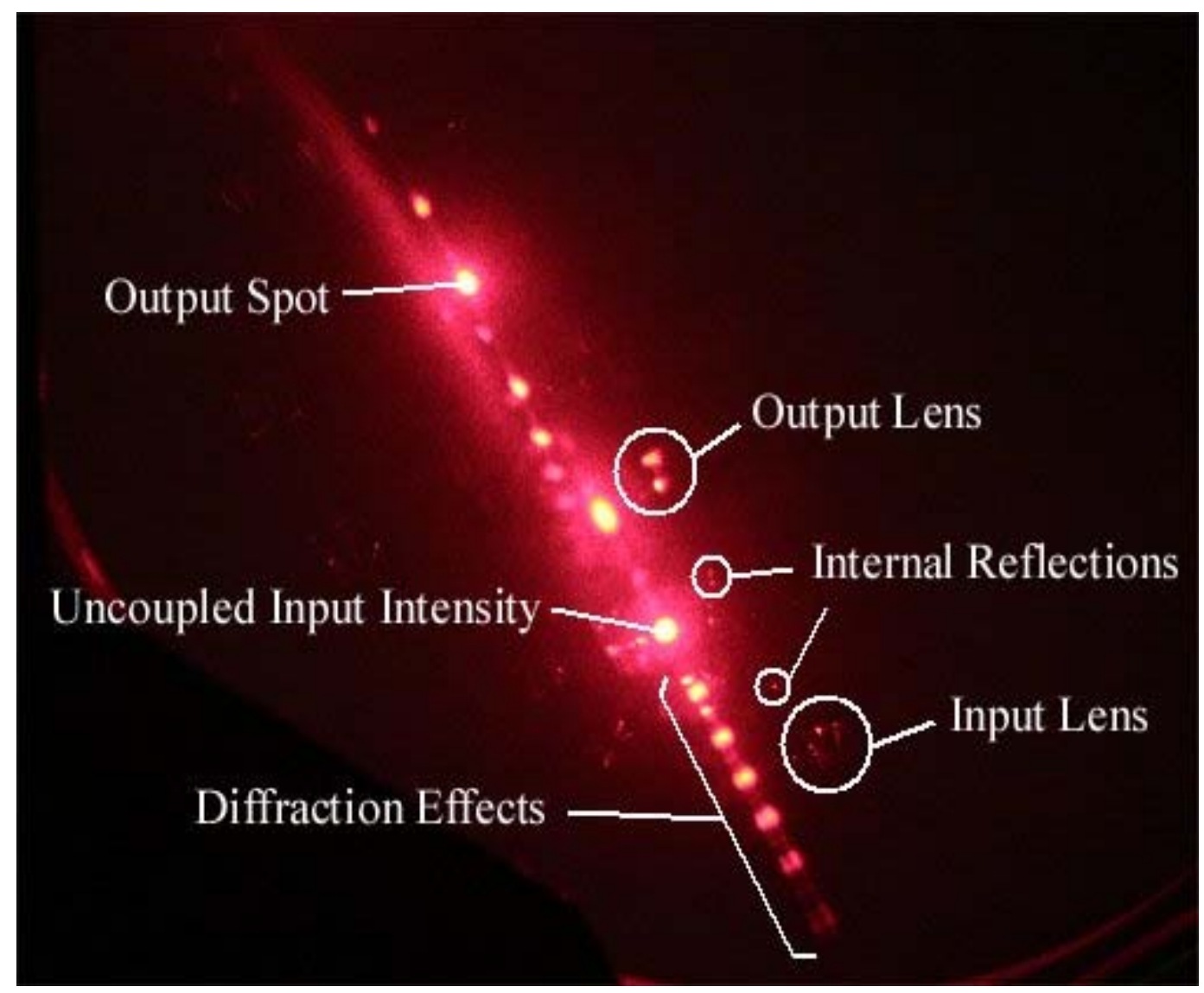

Figure 5.19: Angled view of coupled off-axis beam intensity at $1 \mathrm{~cm}$. Internal reflections are readily visible.

These images show both coupled and uncoupled beam intensities due to the power of the laser used $(\sim 1 \mathrm{~mW})$, as well as diffraction effects caused by the lens pattern. In the angled view, faint internal reflection spots are visible, indicating internal propagation. The output spot experiences diffraction as well, resulting in multiple output intensities. Another effect of off-axis focus can be seen at distances larger than the focal length, asymmetric spot spreading, or comatic aberration. This effect, illustrated in Figure 5.20, is the result of differences in focal length for each zone radius of the off-axis lens geometry, as described in Chapter 2. 

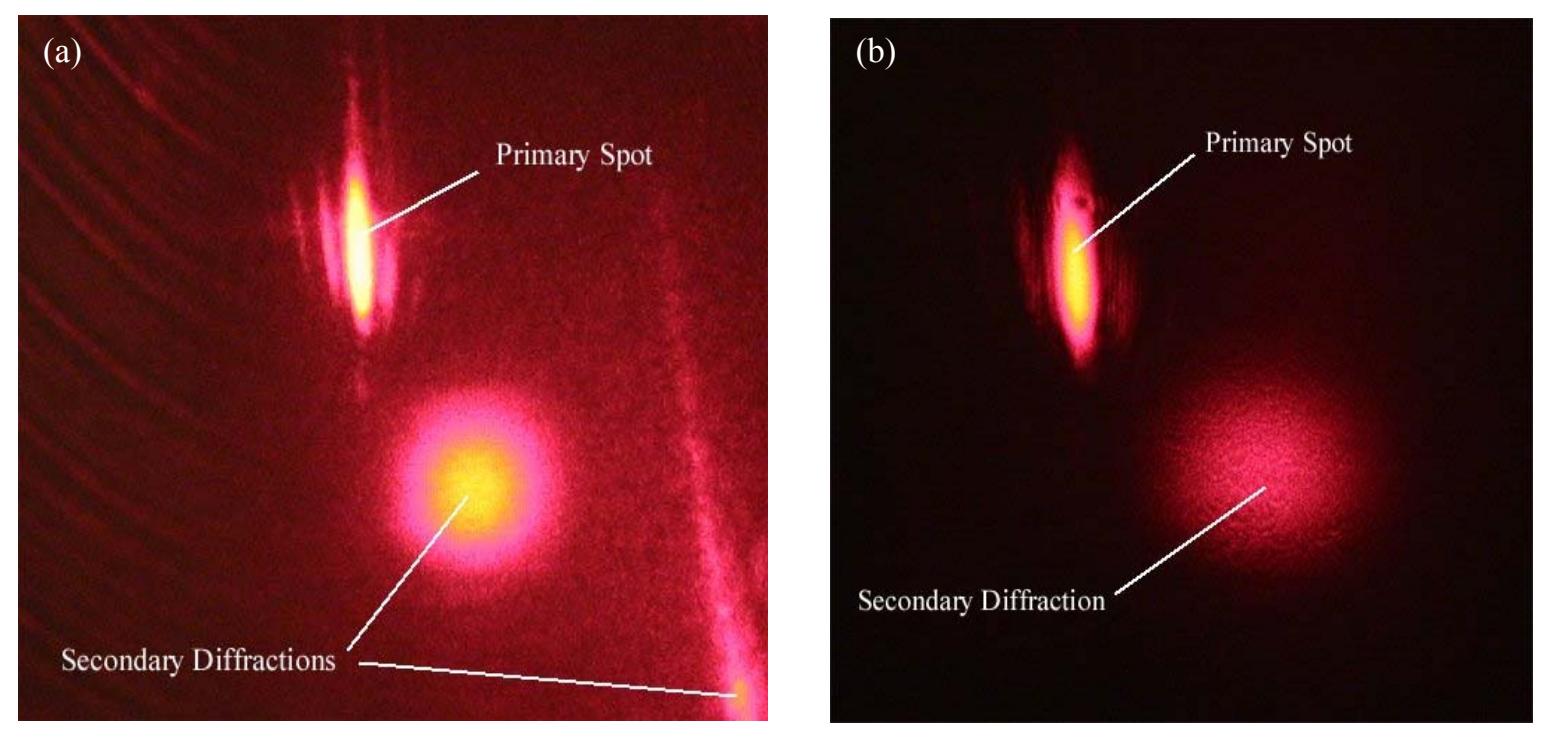

Figure 5.20: Comatic aberration of the output beam at (a) $20 \mathrm{~cm}$ and (b) $40 \mathrm{~cm}$. Secondary diffraction intensities of the input beam are visible.

Even though the spot experienced comatic spreading, output beam intensity was great enough for reflective optical monitoring studies to be performed.

\subsection{MEMS Monitoring Using the Lens Substrate}

In order to use the integrated optical elements to probe the lateral comb resonator devices for motion detection, the optical microprobe setup had to be modified. The through-wafer probing optics were removed to make room for the lens substrate and beam focusing lens. A schematic view of the reflective probing setup can be seen in Figure 5.21. The lens substrate and focusing lens were given independent 3-axis freedom to ease in angular adjustment for achieving the optimum coupling angle, as well as variable input beam positioning to use different input-output path lengths. The detector fiber was given $x, y$, and $z$-axis adjustment as well for maximum coupling of the reflected output signal. The die package adjustment was left unaltered, leaving both fine and coarse 3-axis freedom. 


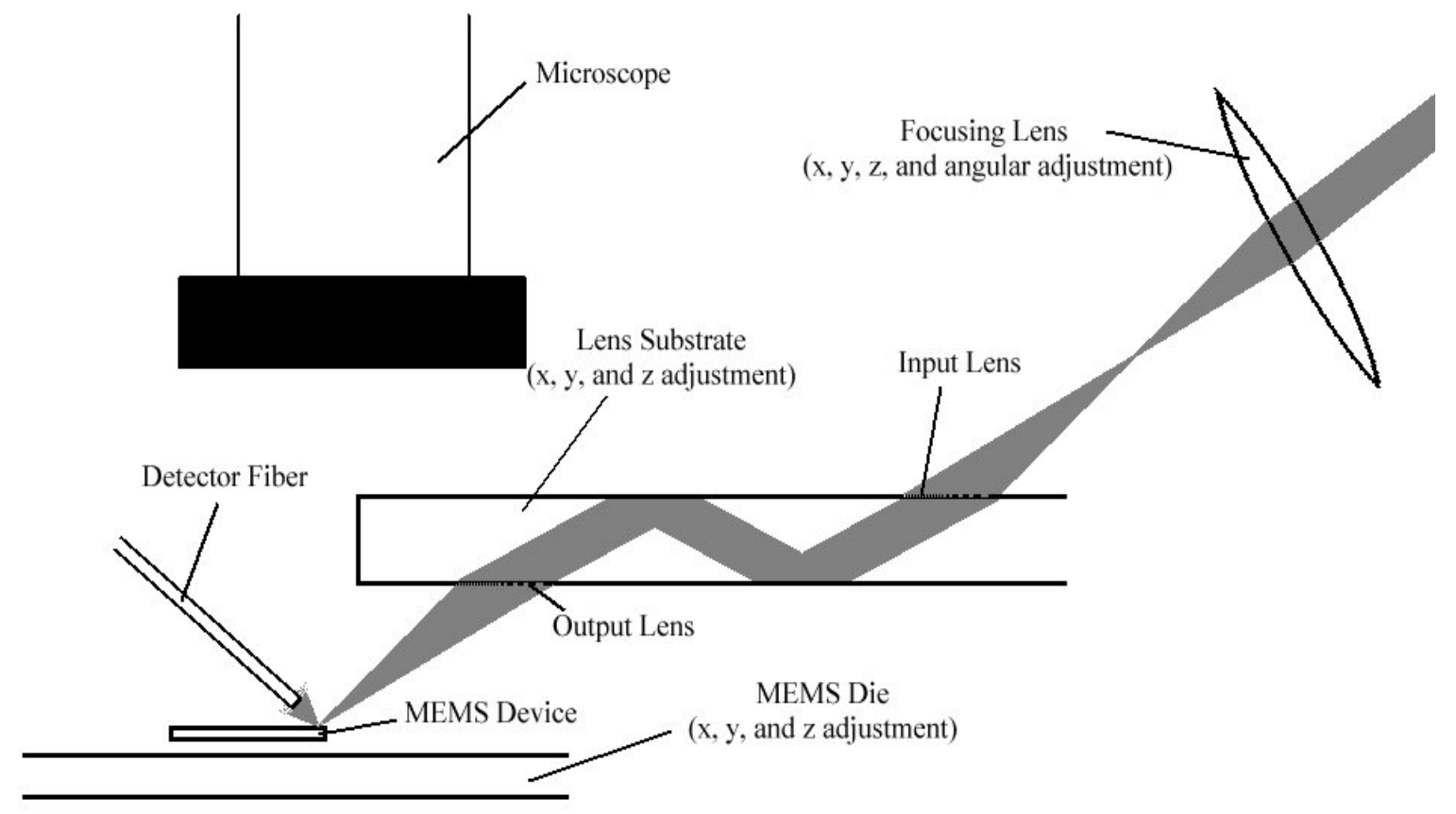

Figure 5.21: Microoptical reflective device monitoring setup.

Figures 5.22 and 5.23 show the actual setup with detector fiber and lens substrate in place.

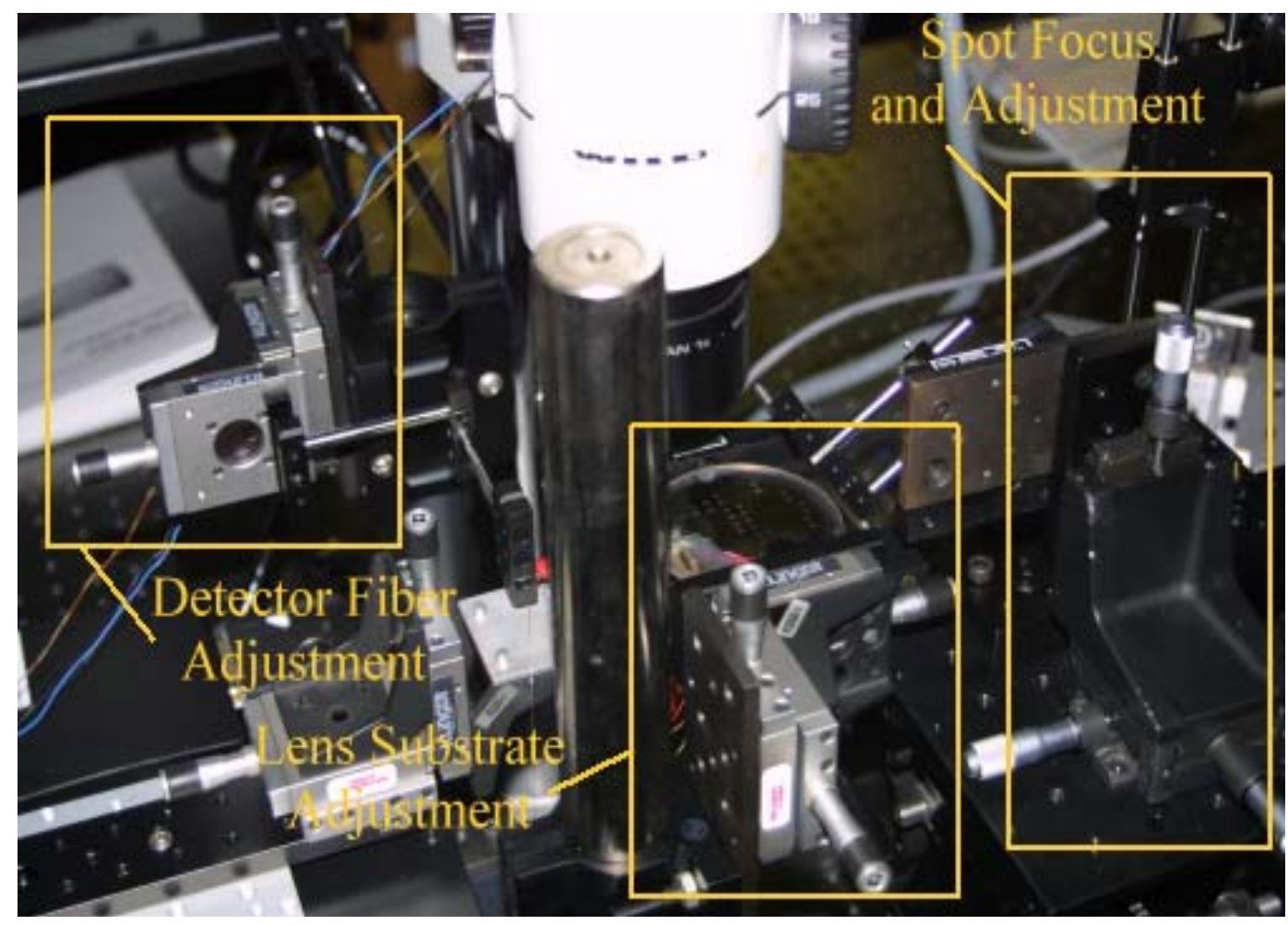

Figure 5.22: Optical microprobe setup with lens substrate added. 


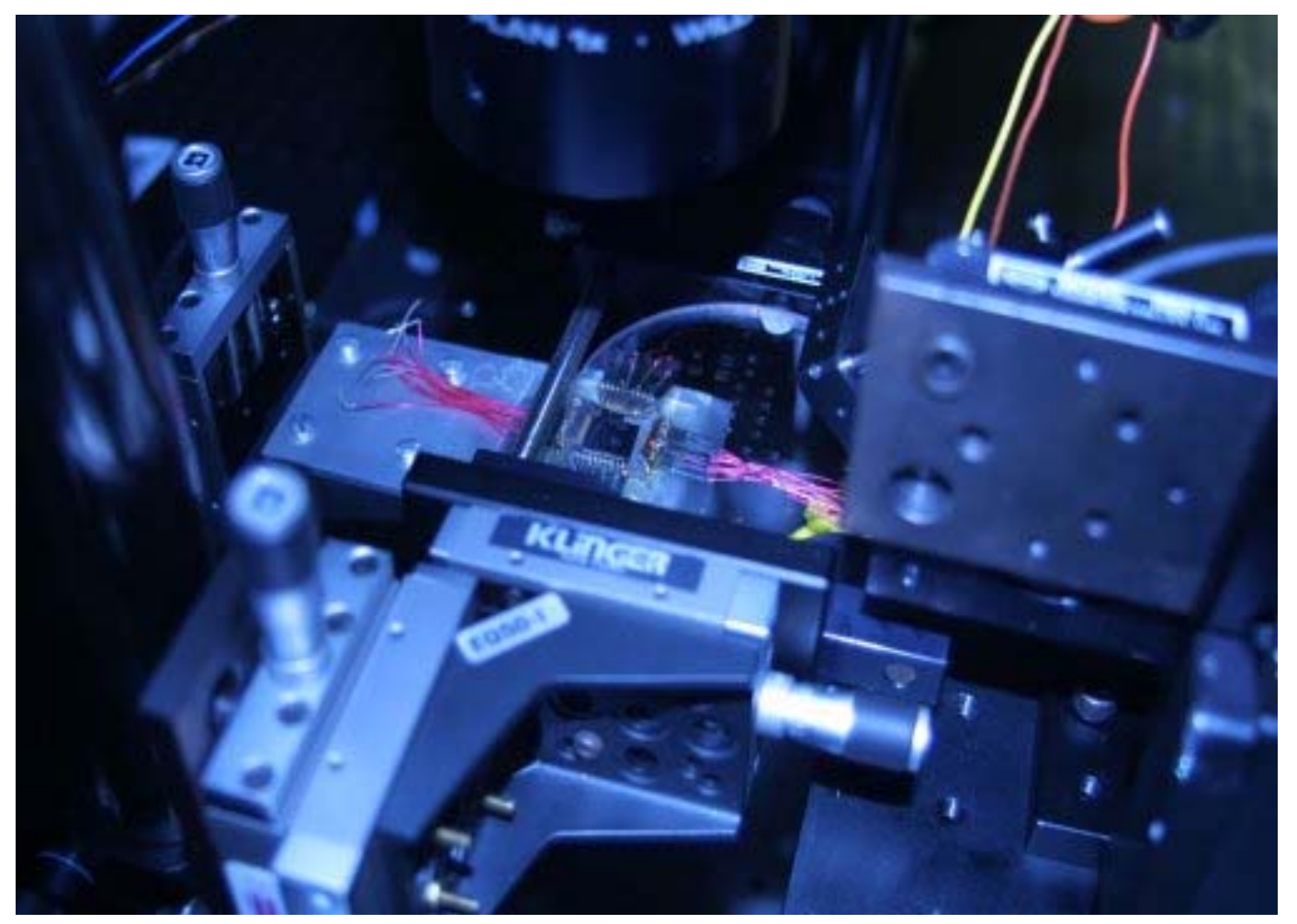

Figure 5.23: Close-up of the package and lens substrate arrangement.

Figure 5.24 pictures the system being used to illuminate a single-opening geometry lateral comb resonator with a $\mathrm{HeNe}$ beam being coupled into the microlens substrate and used as an optical probe beam. A view of the illuminated device can be seen in Figure 5.25. 


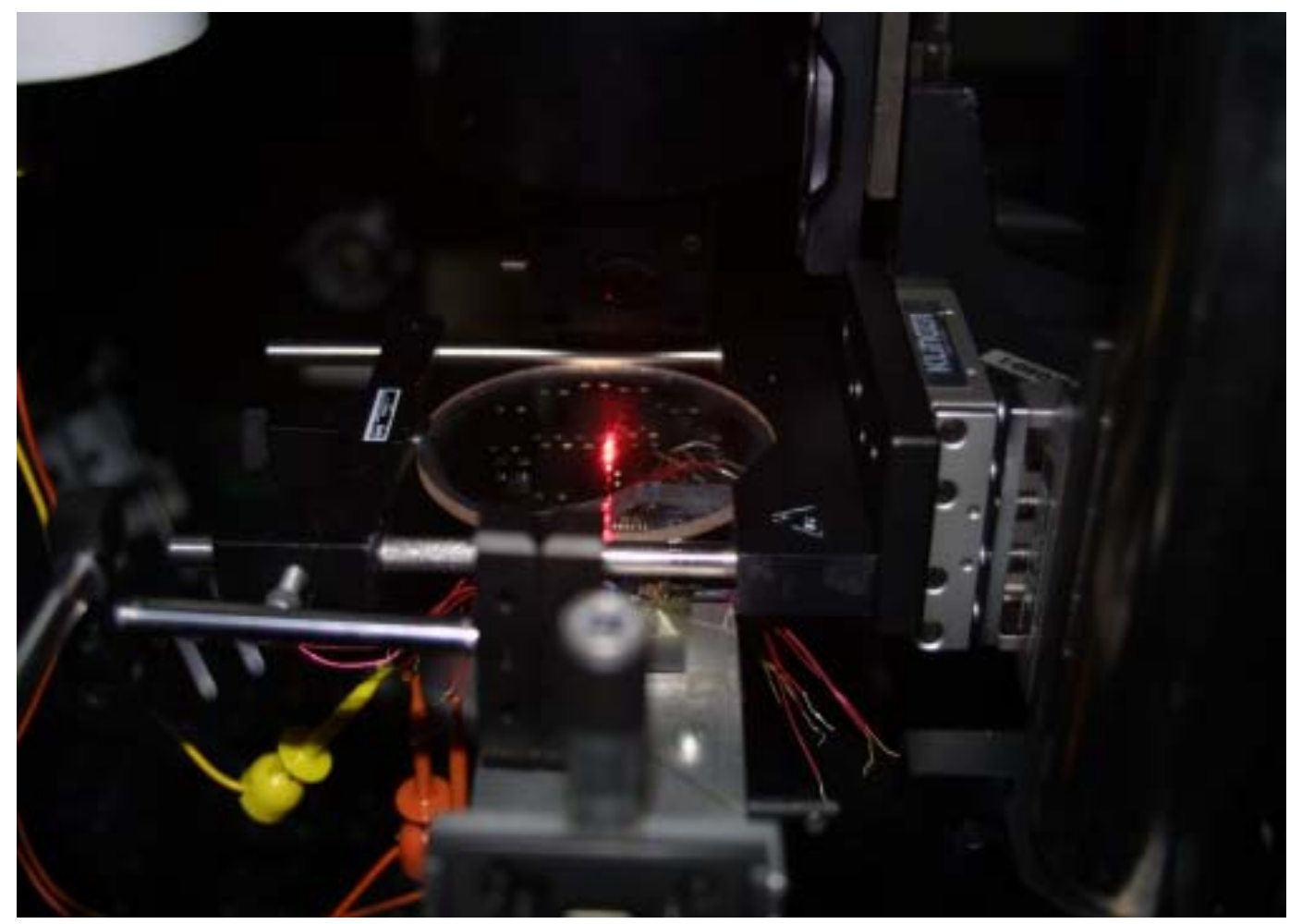

Figure 5.24: The lens substrate in use, illuminating a device on the MEMS die.

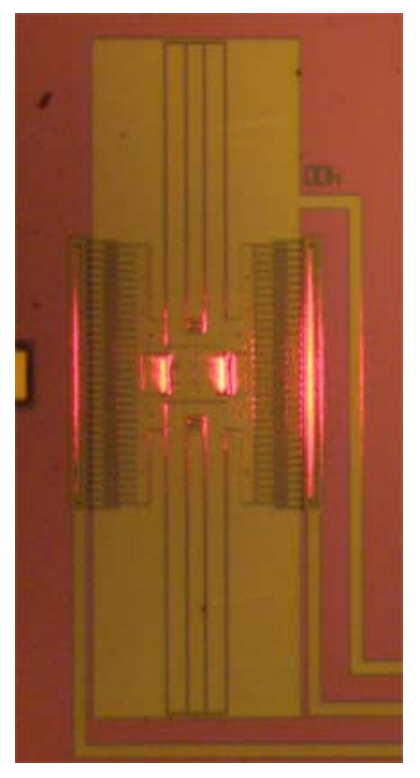

Figure 5.25: A single-opening lateral comb resonator illuminated by the output beam of the lens substrate. 


\section{Chapter 6 Conclusions}

It has been shown that new designs of the MEMS device geometry and optical microprobe setup allow for the application of simple linear closed-loop control routines with acceptable results. The need for detailed positional information, required for robust nonlinear control applications, has been met by using grating structures as a through-wafer optical probing area. Software tools have been developed that examine through-wafer diffraction intensity patterns and create mask layout files of integrated on and off-axis focusing binary Fresnel zone plate lenses. A successful binary diffractive microoptical element fabrication process has been established, and the focusing properties of the microlenses have been examined. The lenses have also been used to direct a visible wavelength probe beam in order to illuminate a lateral comb resonator device. This chapter will summarize results, as well as discuss further research in achieving greater positional resolution of the optical output signal and improving the output beam quality of the integrated optical elements.

\subsection{Through-Wafer Diffraction Study Results}

In order to study the diffraction effects of both the single-opening and the periodic $2 \mu \mathrm{m}$ opening grating translation stage geometries designed for increased positional resolution, a Matlab program was developed to examine the interaction of the complex amplitude of the Gaussian input beam, the complex transmission profile of the stage, and the transfer function of free space. This program is highly flexible and can be modified to accept different stage 
geometries, beam wavelengths and types, and observation plane distances. Theoretical optical output waveforms can also be generated using a Simulink system model of the lateral comb resonator with experimentally determined model parameters (mass, resonance, spring constant) to determine theoretical stage displacement.

When compared with actual experimental optical output data obtained by probing a moving device with a $2 \mu \mathrm{m}$ grating geometry translation stage, theoretical waveforms obtained from the Matlab optical probe field intensity program match in frequency, phase shift, and relative amplitude for sinusoidal drive voltage frequencies ranging from 1-5 kHz. A correlation between stage velocity and peak width can be made as well. At or near the zero, or at-rest position, stage velocity is greatest, resulting in narrow intensity peaks. As the stage reaches the limits of its motion, stage velocity is lower, causing the intensity peaks to become broadened. A positional resolution of $4 \mu \mathrm{m}$ peak-to-peak and $2 \mu \mathrm{m}$ peak-to-valley has been achieved. Near the resonant frequency of the device $(\sim 2.9 \mathrm{kHz}$, Figure 4.35$)$ five distinct intensity peaks can be seen, giving a total stage displacement of $\sim 20 \mu \mathrm{m}$, a value that agrees with simulated displacement results. The secondary sinusoidal frequency in the experimental data has been considered and may be an effect of vertical stage motion, but is more likely due to large spot size, causing an interaction of the probe beam with non-grating areas of the device stage.

By decreasing the grating size to $1 \mu \mathrm{m}$, positional resolution should increase accordingly, however, due to fabrication process limitations, linewidths less than $2 \mu \mathrm{m}$ are not resolvable with the MUMPS fabrication process. Multiple detector fibers could be employed to receive multiple intensity peaks of the MEMS motion induced grating diffraction pattern, increasing the optical signal position resolution in the area between intensity maxim and minima. A similar method involving multiple probe beams, each with its own detector, could be used in the same manner. Both of these methods would be a challenge to implement in the current optical microprobe setup due to the limited space in the probing area of the device, but could be realized in an integrated optical monitoring environment. 


\subsection{Integrated Binary Fresnel Lens Conclusions}

Fresnel Zone plate theory has been used to create a program that can be used to produce binary integrated zone plate diffraction pattern layouts. These layouts have been arranged to create a microoptical element/slab waveguide system that can be used to deliver an optical probe beam to a single device in a microelectromechanical system. Off-axis binary lenses have been designed to accept a non-normal, divergent input beam. The lens collimates the input for propagation in the substrate, and further increases the angle of propagation by 10 or 15 degrees to meet the total internal reflection requirements of the waveguide. Output lenses of the same type have been designed and positioned on the opposite surface of the quartz substrate to couple the beam out of the waveguide and focus the beam $0.5-1 \mathrm{~cm}$ for an optical probe beam wavelength of $1310 \mathrm{~nm}$ (1 to $2 \mathrm{~cm}$ for HeNe wavelengths). This focused spot can then be used to optically monitor moving structures on a MEMS device.

A successful diffractive optical microelement photolithography process has been established, providing the ability to resolve photoresist linewidths down to $\sim 1.5 \mu \mathrm{m}$ on quartz substrates using AZ5206-E photoresist and the MA6 mask aligner operating at $320 \mathrm{~nm}$. A reactive ion etch process has been developed using Fluoroform at a flow of $110 \mathrm{sccm}$, a process pressure of $30 \mathrm{mT}$, and $150 \mathrm{~W}$ of RF power for 40 minutes. These parameters result in successful transfer of the photoresist pattern to the quartz substrate with an etch depth of $\sim 450 \mathrm{~nm}$.

Minimal photoresist loss is evident, mainly on smaller features (larger zone radii) that contribute little to the overall diffraction of the beam. This photoresist loss is due to the length of time require to reach the desired etch depth. To eliminate PR during the etch process (and thus its loss), different mask materials, such as metals, could be used, but lateral undercutting of the masking material will still be a problem. If the mass flow controller malfunction the RIE is corrected, different gas flow rates could be studied as well to determine the effect of gas flow on etch rate, possibly shortening the process time. Due to the increased vertical directionality of the ICP etch process, using ICP power could also reduce PR loss as well as increase the anisotropy of the etch.

The fabricated binary microoptical elements were qualitatively examined and shown to have the expected focusing qualities. For visible light $(632 \mathrm{~nm})$, focal lengths of $1-2 \mathrm{~cm}$ were 
observed for lenses designed for focusing at $0.5-1 \mathrm{~cm}$ using infrared wavelengths. Off-axis lenses caused comatic aberration of the coupled output beam intensity pattern. Even though significant coma was present, the lens substrate was placed in the optical microprobe setup and used to illuminate a lateral comb resonator device, showing that integrated optical elements can be used to deliver an optical probe beam to MEMS devices for monitoring purposes.

This research resulted in an integrated optical substrate that can be used as a flexible research tool with which to examine the usefulness of employing integrated optics for MEMS device monitoring. Future research must focus on eliminating comatic and other aberrations by using multi-level patterns and complex diffraction pattern geometries developed by starting with the desired spot pattern and working backwards to the diffractive element using statistical optics and holography. These sophisticated patterns could also be designed to probe multiple devices at once, allowing multiple probes to monitor one device, or multiple devices simultaneously, increasing the effectiveness of the integrated optical monitoring system. Future efforts must also include a study of packaging methods that will be used to fabricate the complete IOM-MEMS system. If the types of assembly and packaging methods used are more likely to fail than the device itself, package failure will outweigh the benefits of using such a system for lifetime monitoring and control of MEMS will be lost. 


\section{Appendix A Lens Design and Optical Analysis Programs}




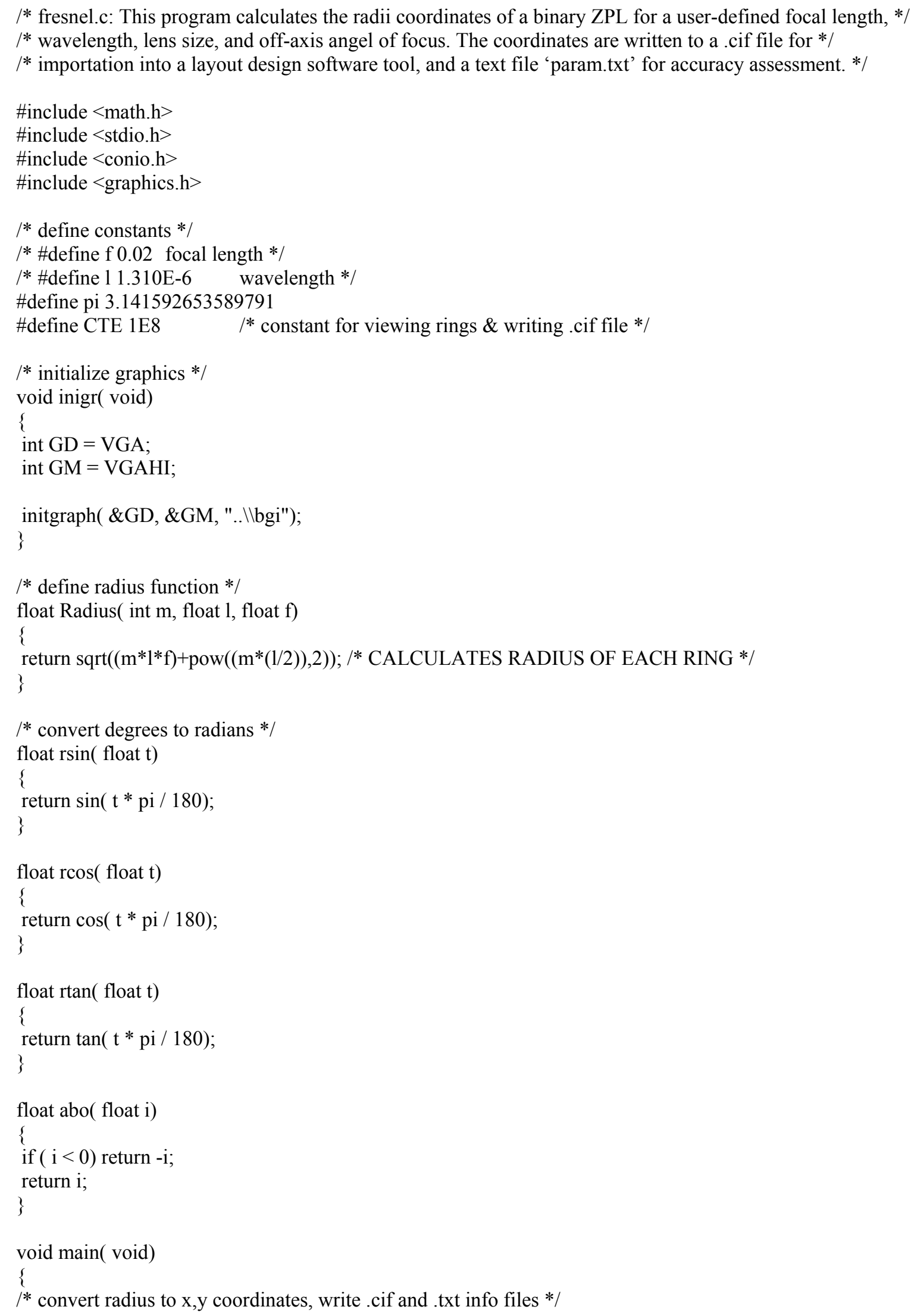




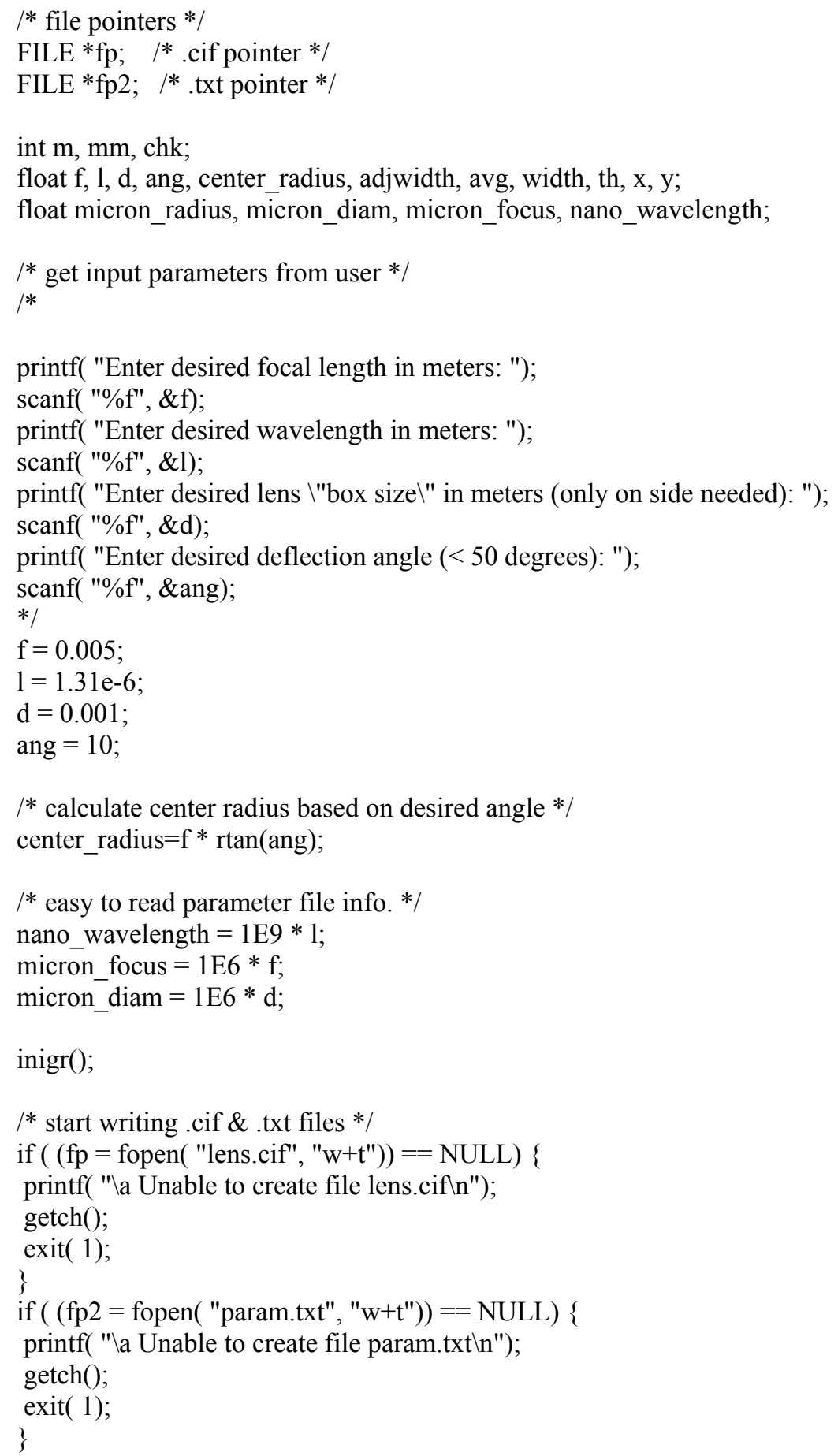




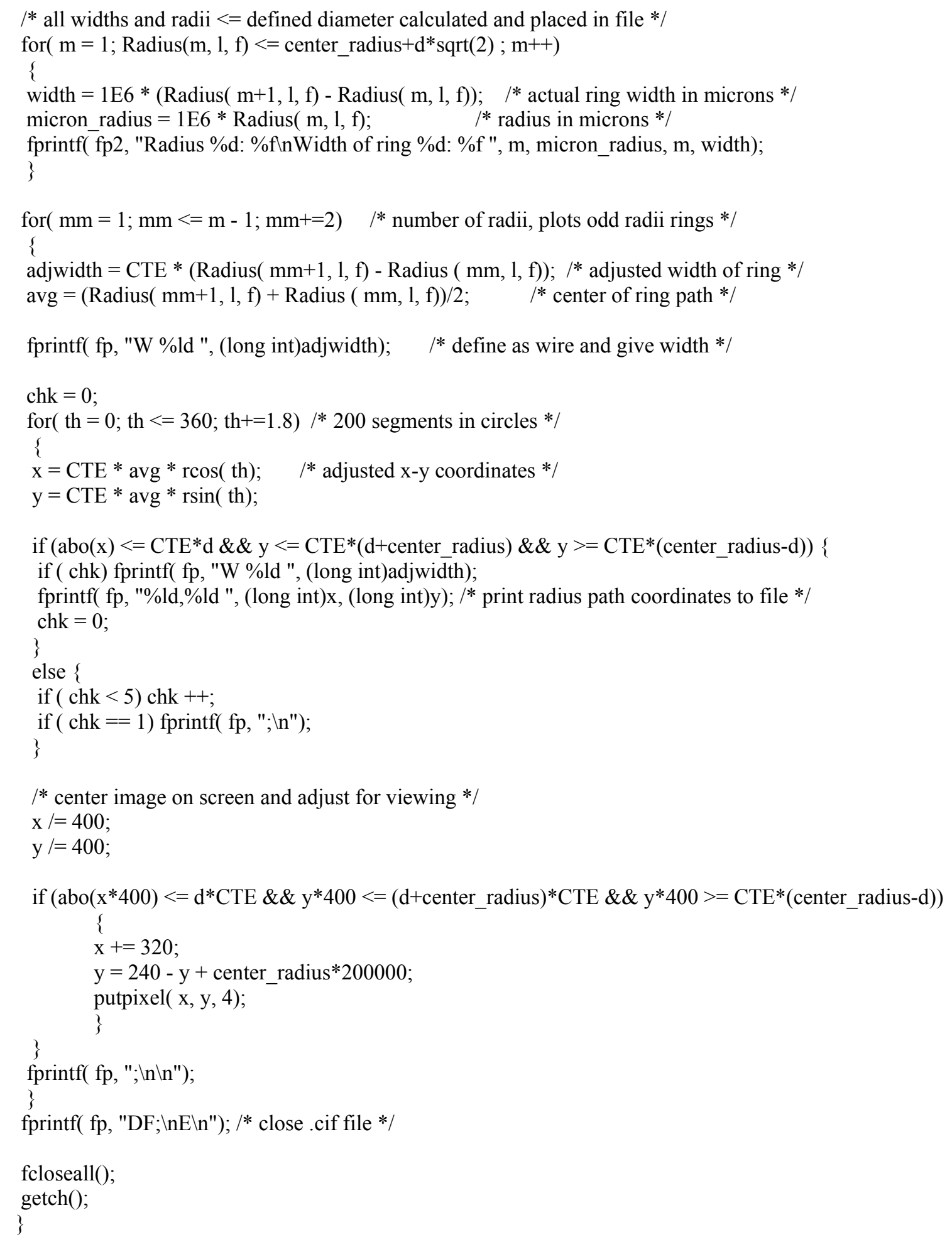




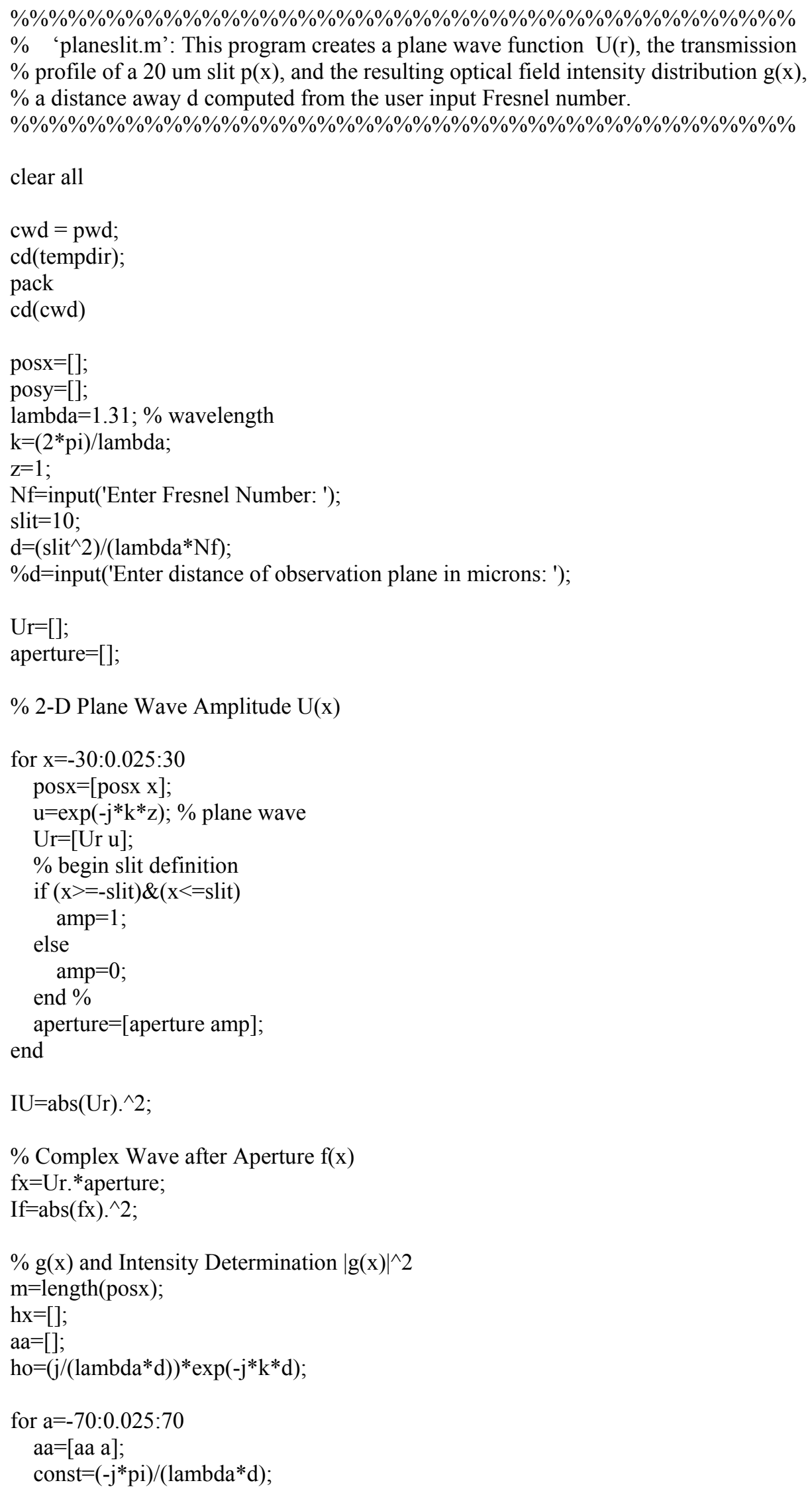




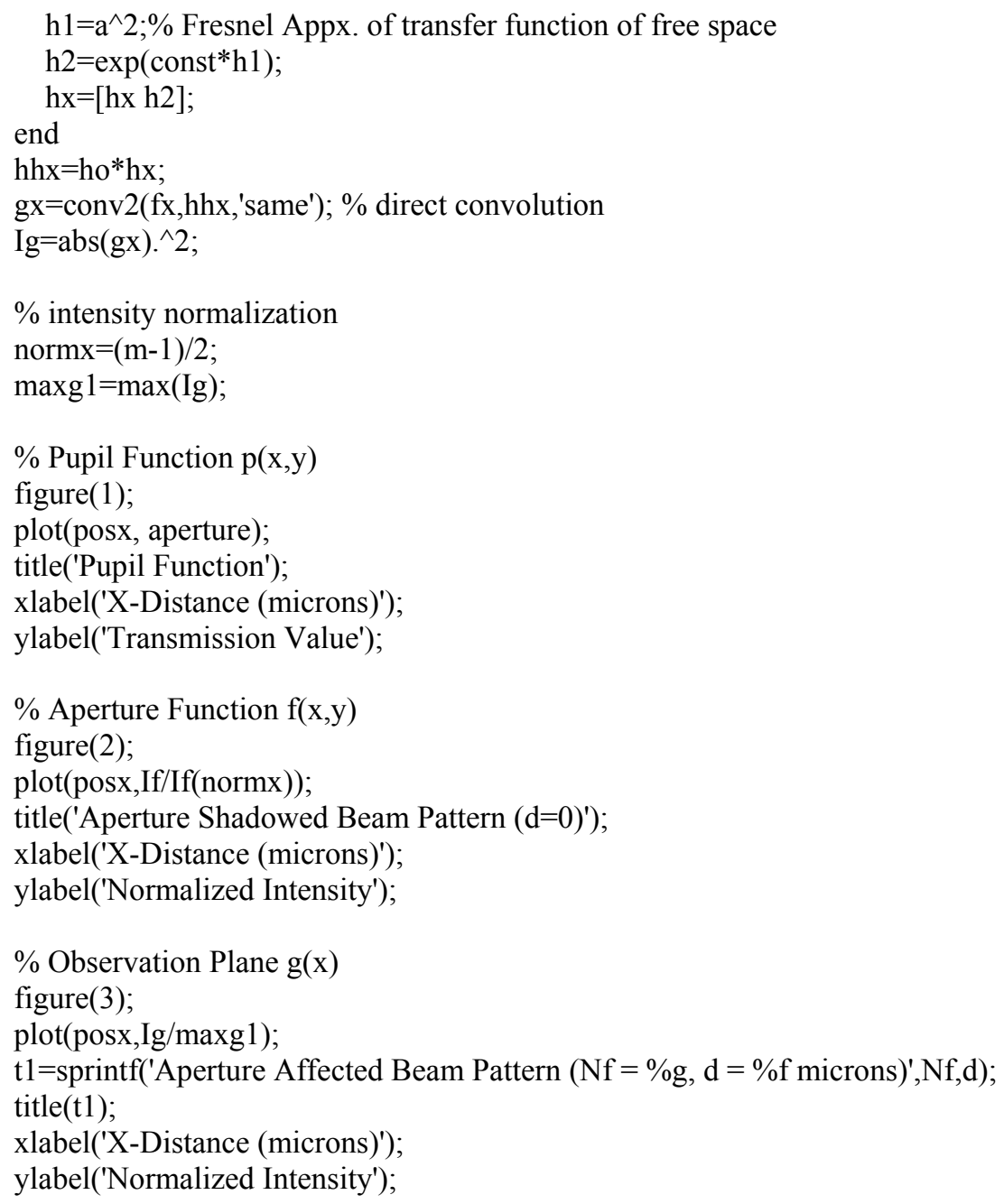


\%\%\%\%\%\%\%\%\%\%\%\%\%\%\%\%\%\%\%\%\%\%\%\%\%\%\%\%\%\%\%\%\%\%\%\%\%\%\%\%\%\%

$\% \quad$ 'planerect.m': This program creates a plane wave function U(r), the transmission

$\%$ profile of a 10x30 um rectangular opening $\mathrm{p}(\mathrm{x}, \mathrm{y})$, and the resulting optical field

$\%$ intensity distribution, $\mathrm{g}(\mathrm{x}, \mathrm{y})$, a distance away $\mathrm{d}$ computed from the user input

$\%$ Fresnel number.

\%\%\%\%\%\%\%\%\%\%\%\%\%\%\%\%\%\%\%\%\%\%\%\%\%\%\%\%\%\%\%\%\%\%\%\%\%\%\%\%\%

clear all

$\mathrm{cwd}=\mathrm{pwd}$

cd(tempdir);

pack

cd(cwd)

$\operatorname{pos} x=[] ;$

posy $=[]$;

lambda $=1.31 ; \%$ wavelength

$\mathrm{k}=(2 * \mathrm{pi}) / \mathrm{lambda}$;

$\mathrm{z}=1$;

$\mathrm{Nf}=$ input('Enter Fresnel Number: ');

slit $=5$;

$\mathrm{d}=\left(\operatorname{slit}^{\wedge} 2\right) /($ lambda*Nf$)$;

$\% \mathrm{~d}=$ input('Enter distance of observation plane in microns: ');

ur=[];

$\mathrm{Ur}=[]$

apval=[];

aperture $=[]$

area $=[]$;

\% 3-D Complex Plane Wave Amplitude U(x,y)

for $x=-20: 0.2: 20$

posx $=[$ posx x];

for $y=-20: 0.2: 20$

$\mathrm{u}=\exp \left(-\mathrm{j} * \mathrm{k}^{*} \mathrm{z}\right)$;

$\mathrm{ur}=[\mathrm{ur} \mathrm{u}]$;

$\%$ begin aperture function definition (piecewise)

if $(\mathrm{x}>=-$ slit $) \&(\mathrm{x}<=$ slit $) \&(\mathrm{y}>=-15) \&(\mathrm{y}<=15) \%$ rectangular opening $\mathrm{amp}=1$;

else

$\mathrm{amp}=0$;

end $\%$ end aperture function definition

apval=[apval amp];

end

$\mathrm{Ur}=[\mathrm{Ur} ; \mathrm{ur}]$;

$\mathrm{ur}=[\mathrm{]}$;

aperture $=[$ aperture; apval];

apval $=[]$

end

$\mathrm{IU}=(\operatorname{abs}(\mathrm{Ur})) . \wedge 2$

posy $=-20: 0.2: 20$;

$\%$ Complex Wave after Aperture $\mathrm{f}(\mathrm{x}, \mathrm{y})$

fxy=Ur.*aperture; 


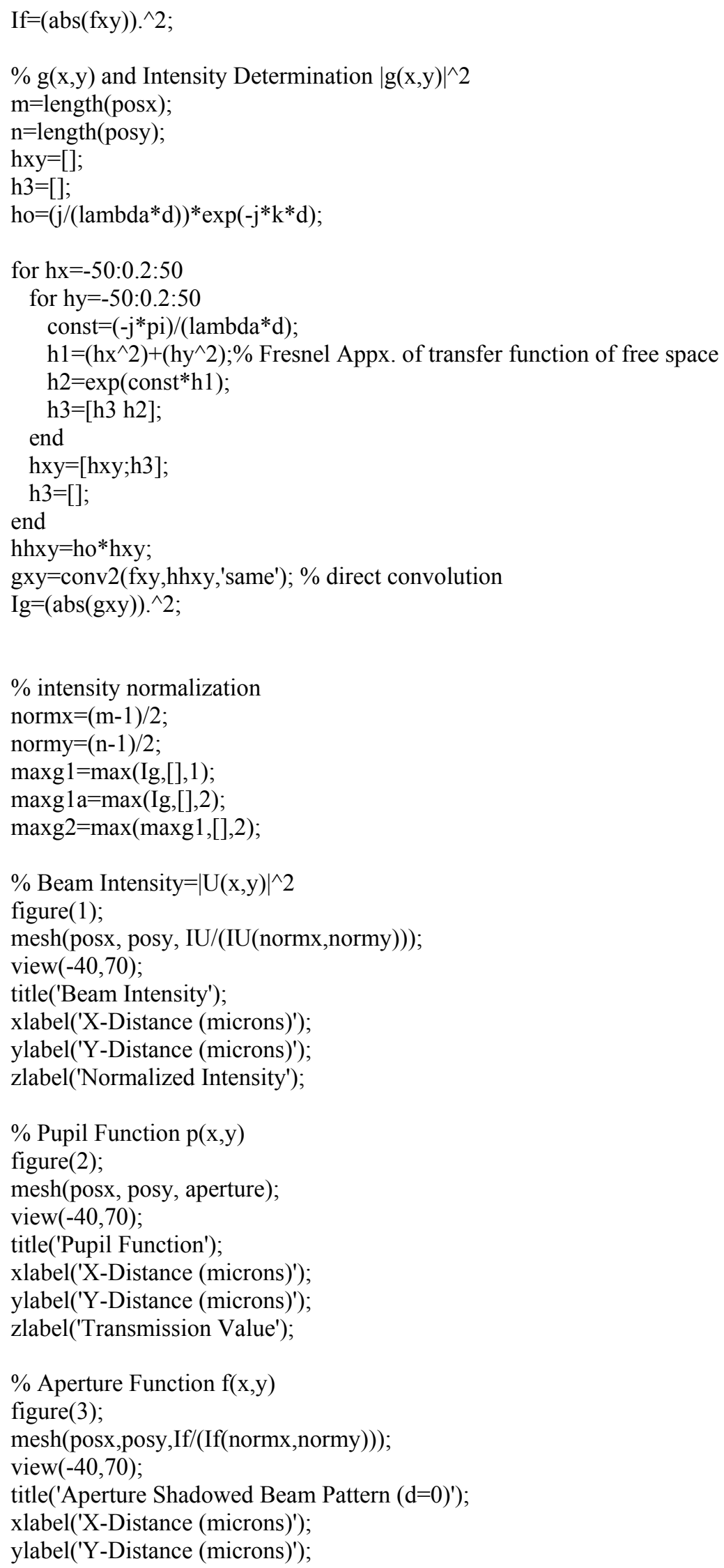


zlabel('Normalized Intensity');

$\%$ Observation Plane $\mathrm{g}(\mathrm{x}, \mathrm{y})$, side view

figure(4);

mesh(posx,posy, Ig/maxg2);

view $(-75,60)$;

$\mathrm{t} 1=$ sprintf('Aperture Affected Beam Pattern ( $\mathrm{Nf}=\% \mathrm{f}, \mathrm{d}=\% \mathrm{f}$ microns)',Nf,d);

title(t1);

xlabel('X-Distance (microns)');

ylabel('Y-Distance (microns)');

zlabel('Normalized Intensity'); 


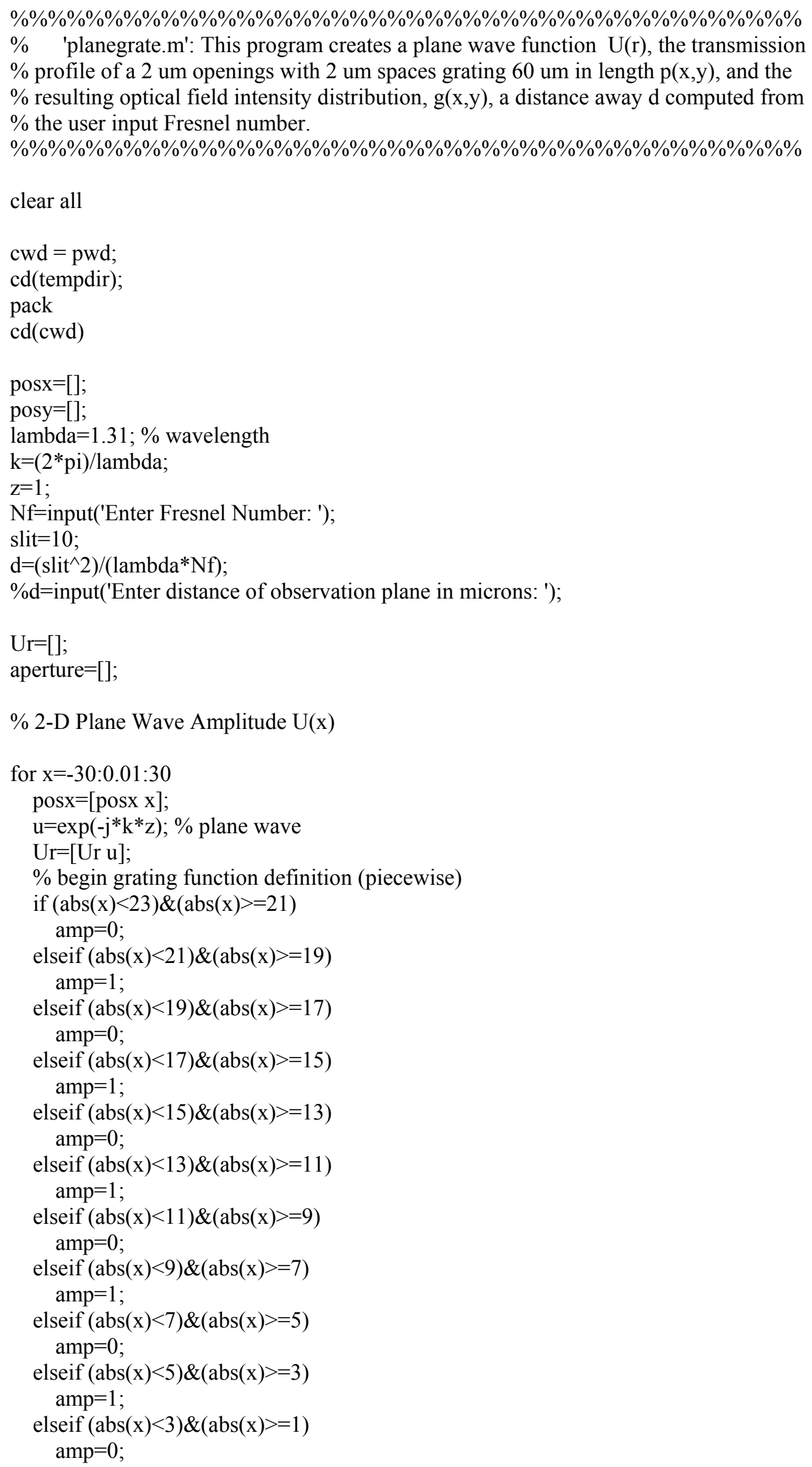




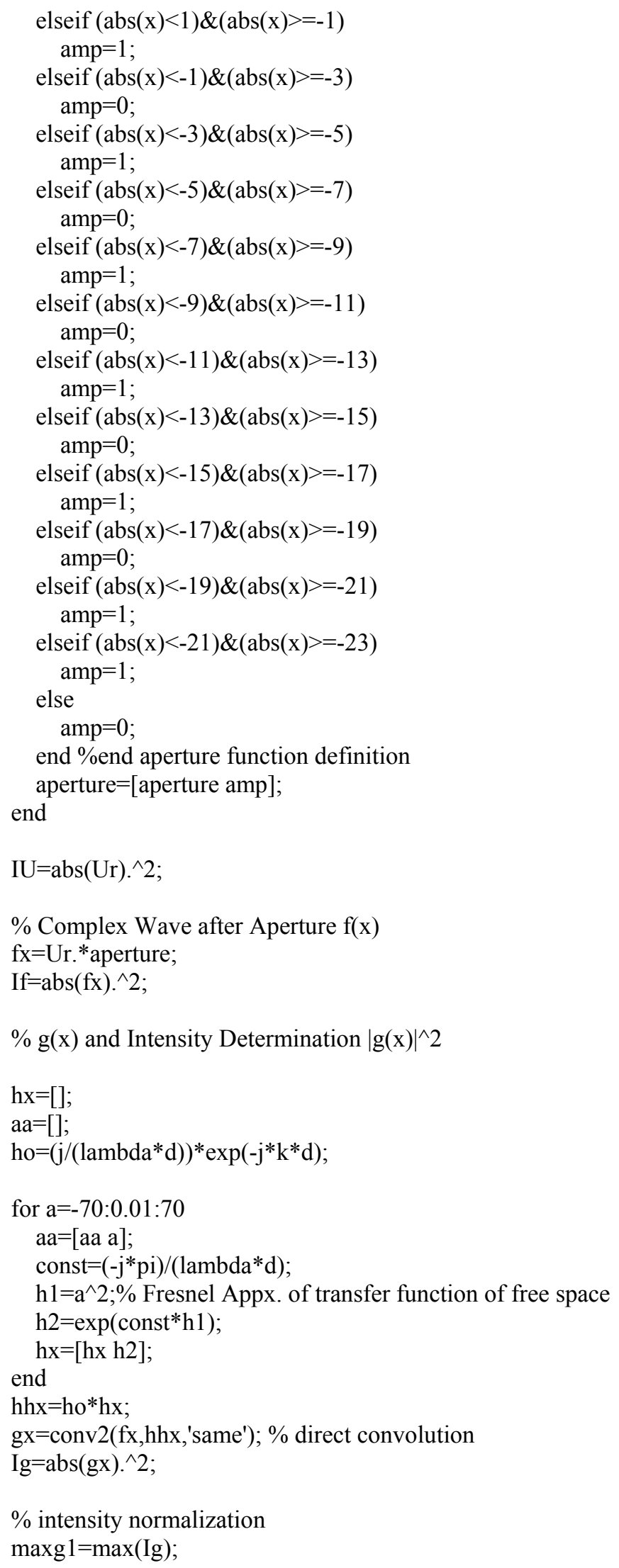


\% Observation Plane $\mathrm{g}(\mathrm{x})$

figure(4);

plot(posx,Ig/maxg1);

$\mathrm{t} 1=\operatorname{sprintf('Aperture~Affected~Beam~Pattern~}(\mathrm{d}=\%$ f microns)',d);

title(t1);

xlabel('X-Distance (microns)');

ylabel('Normalized Intensity'); 


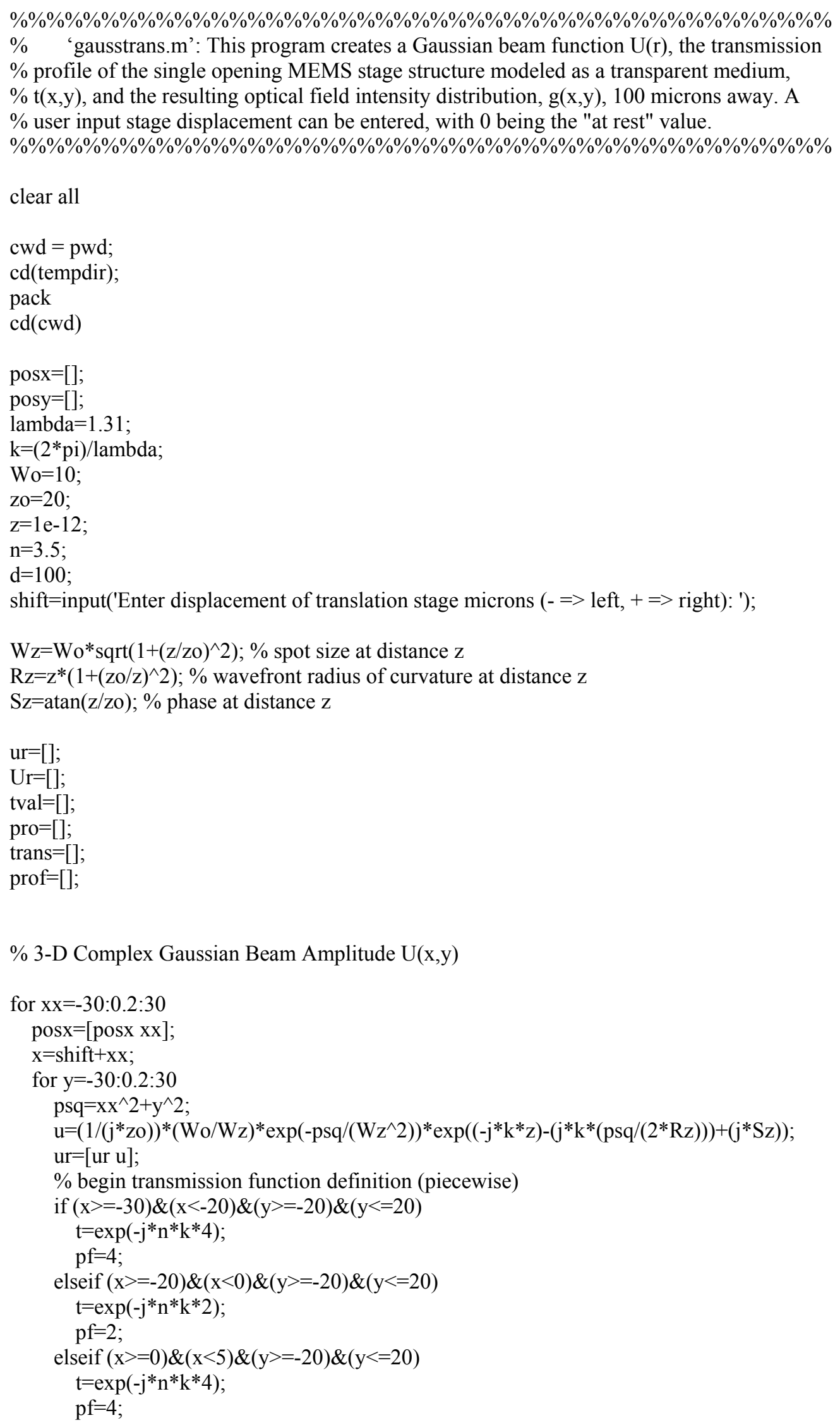




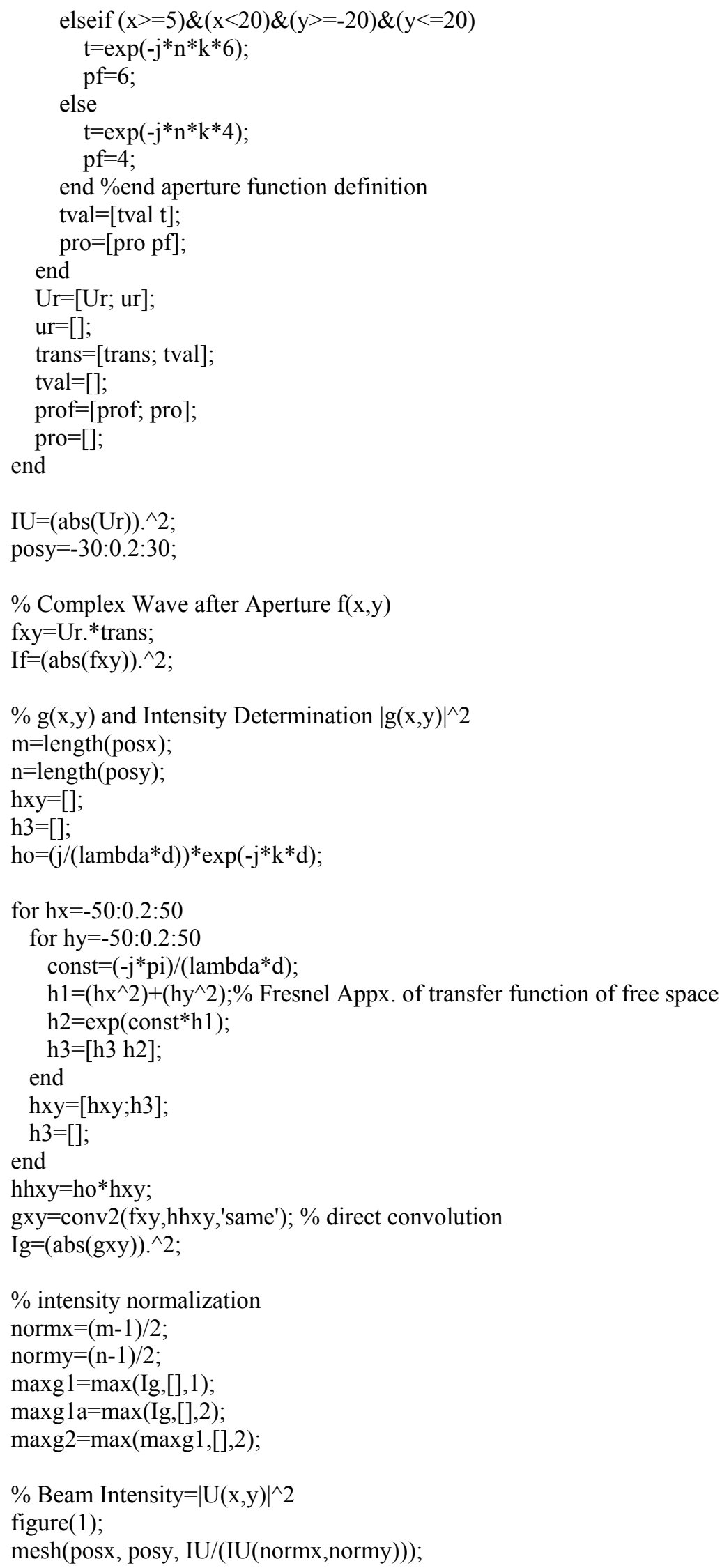




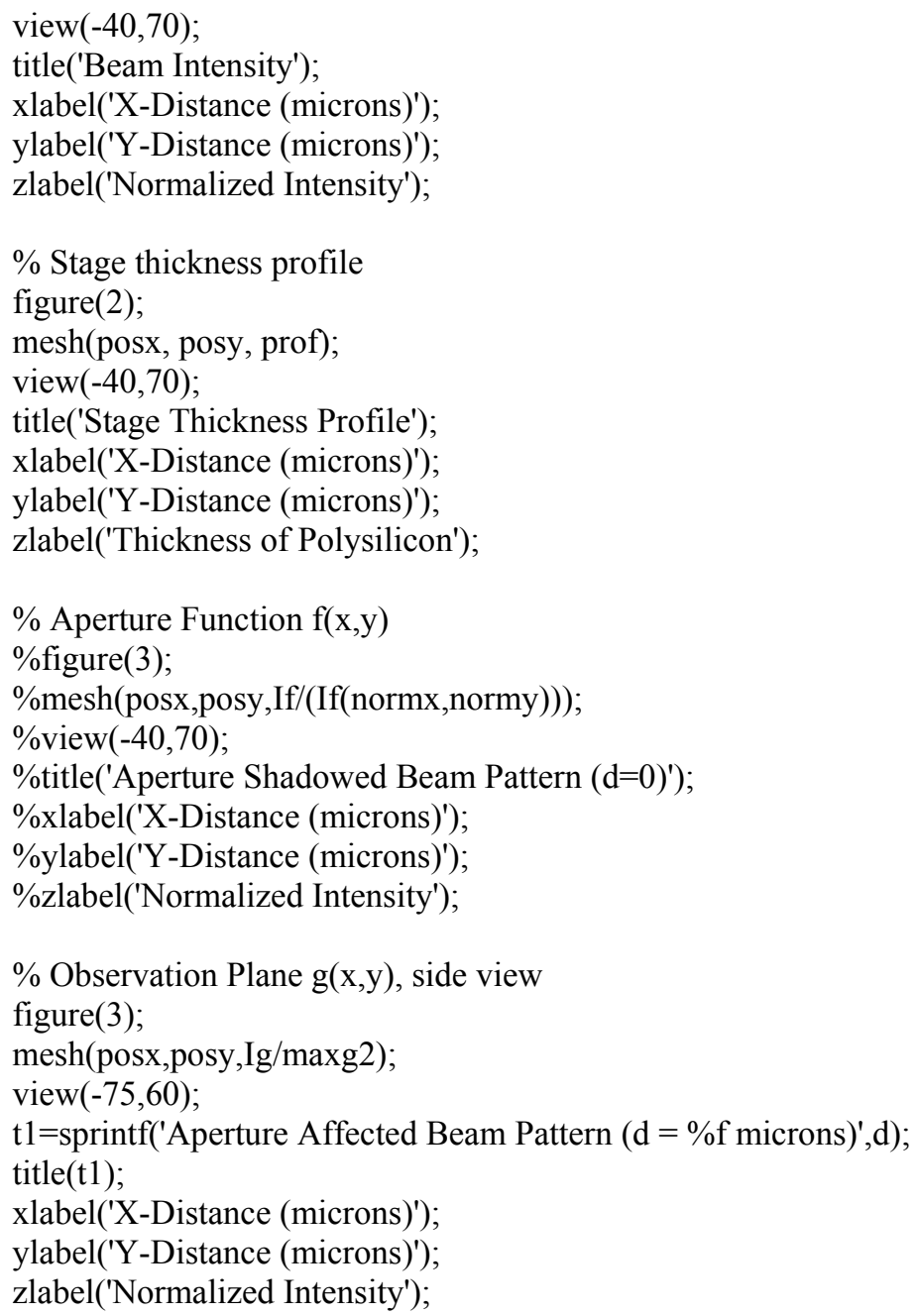




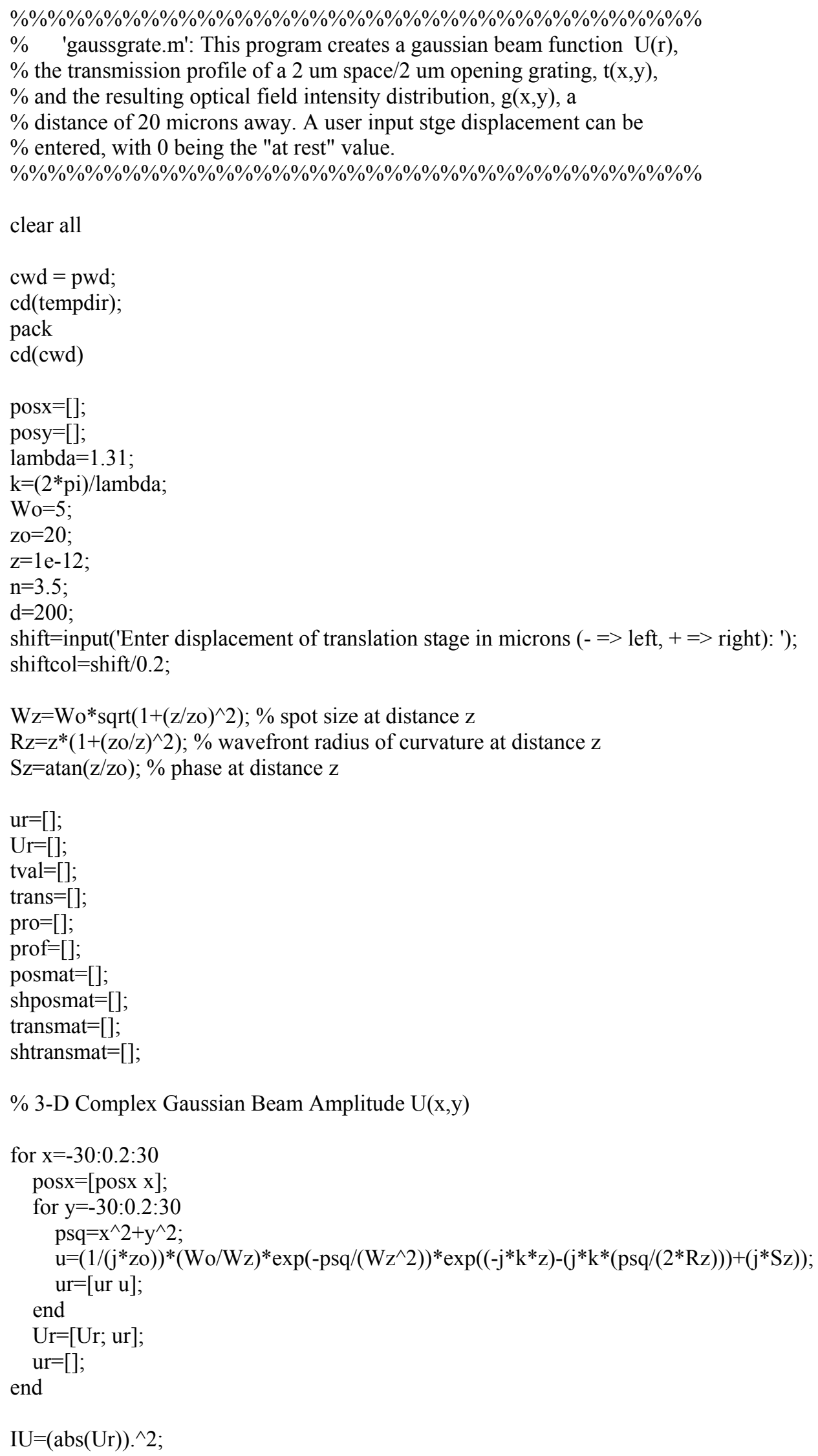




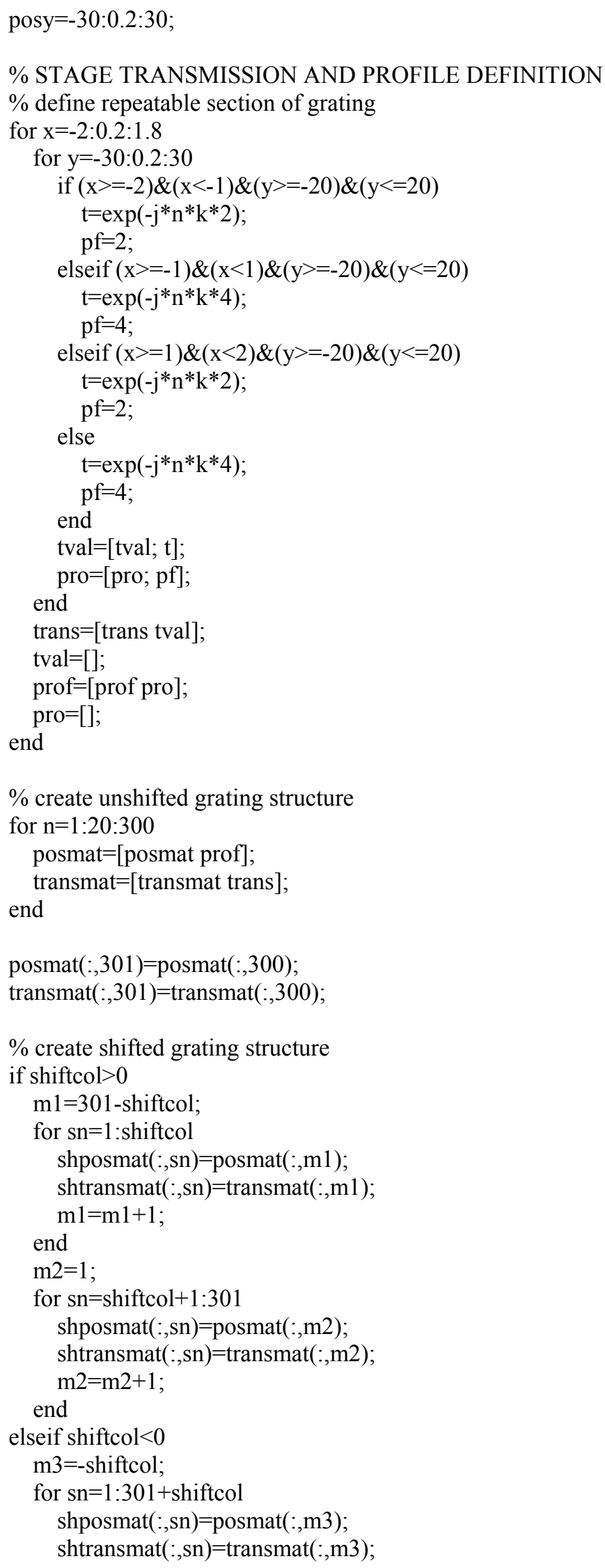




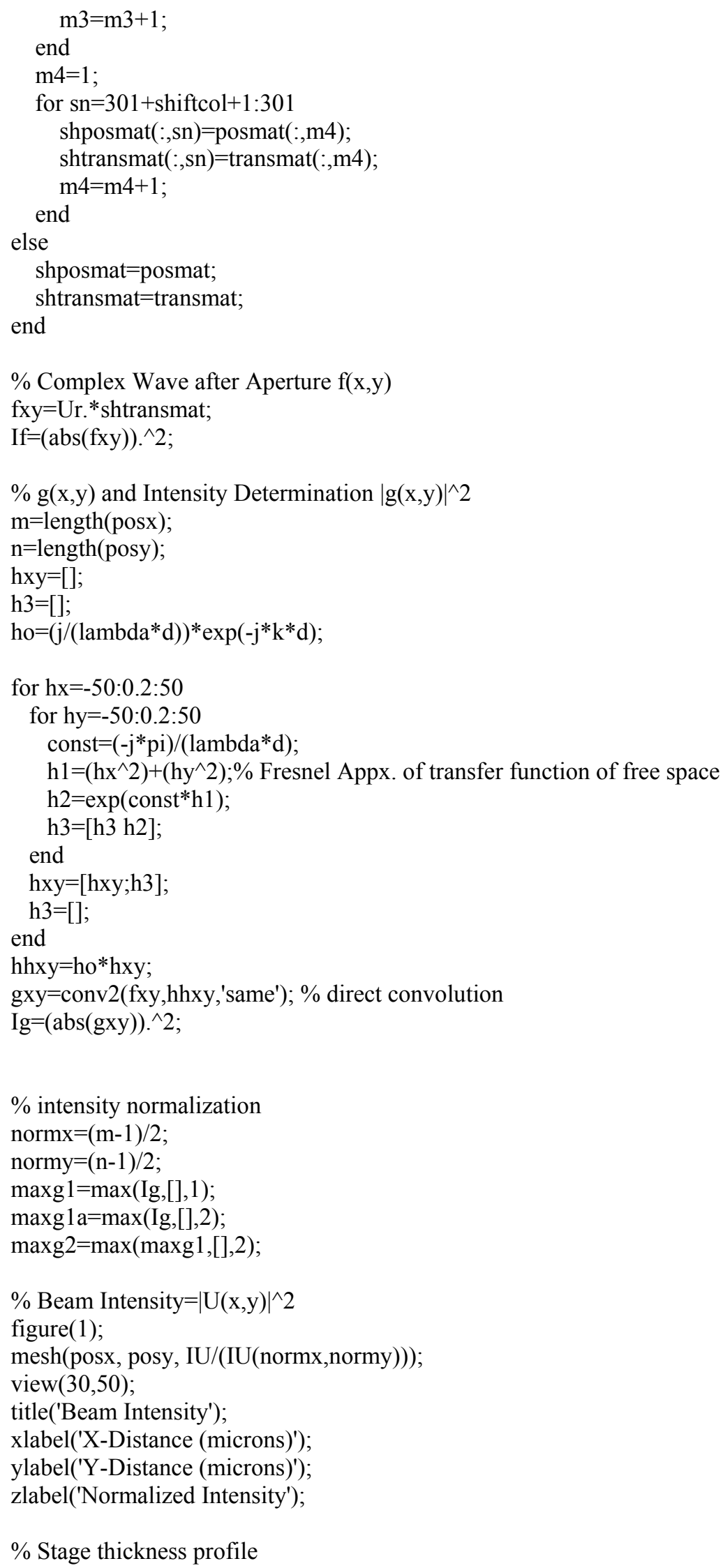




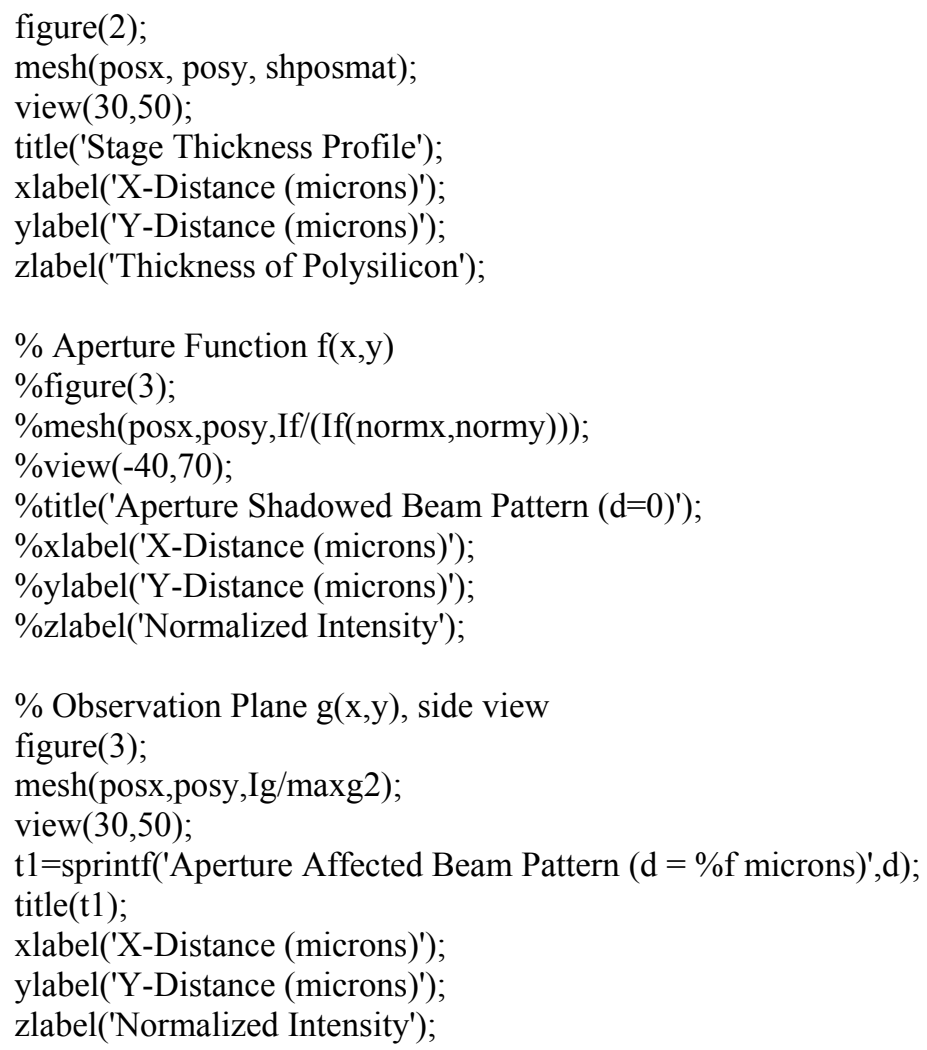




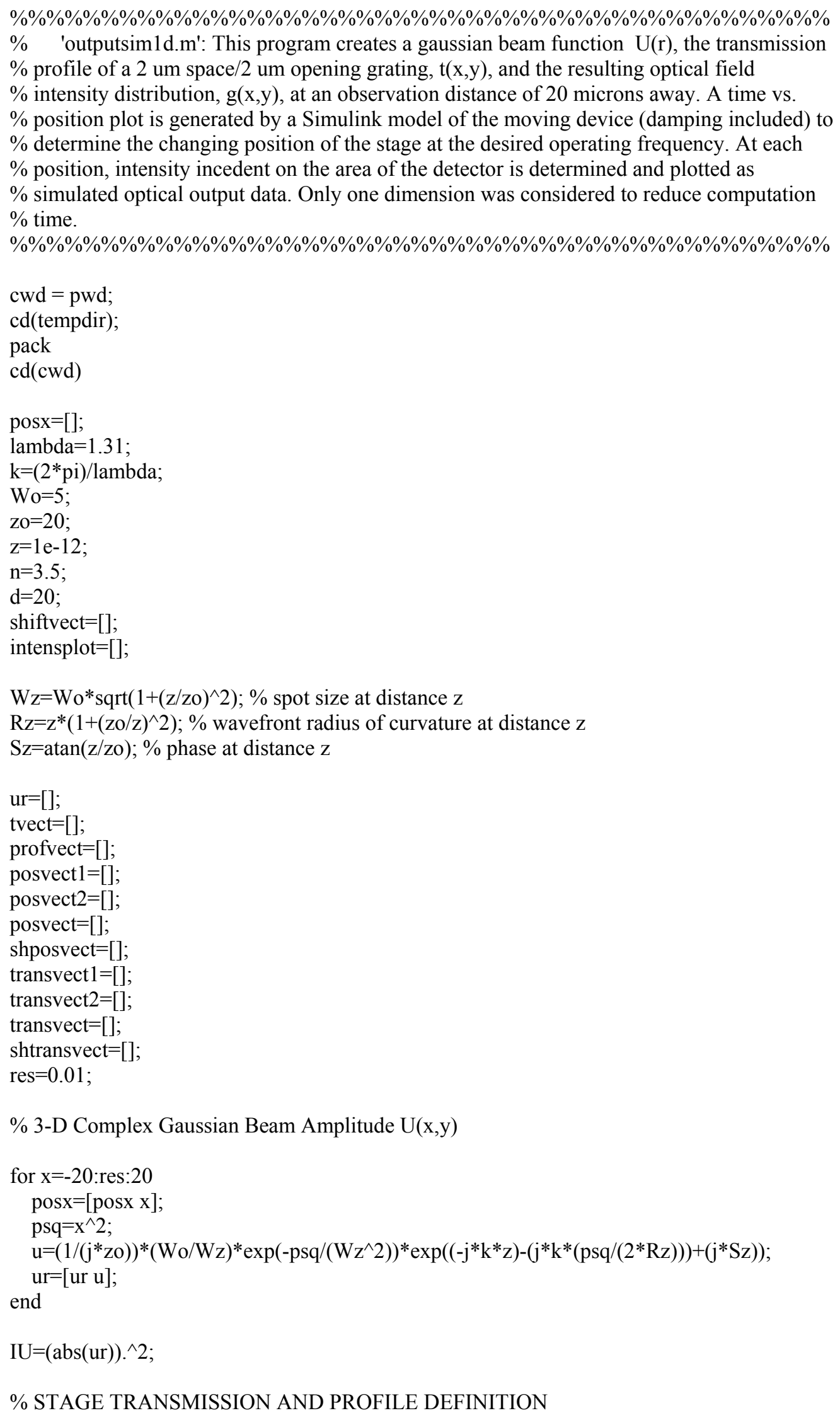




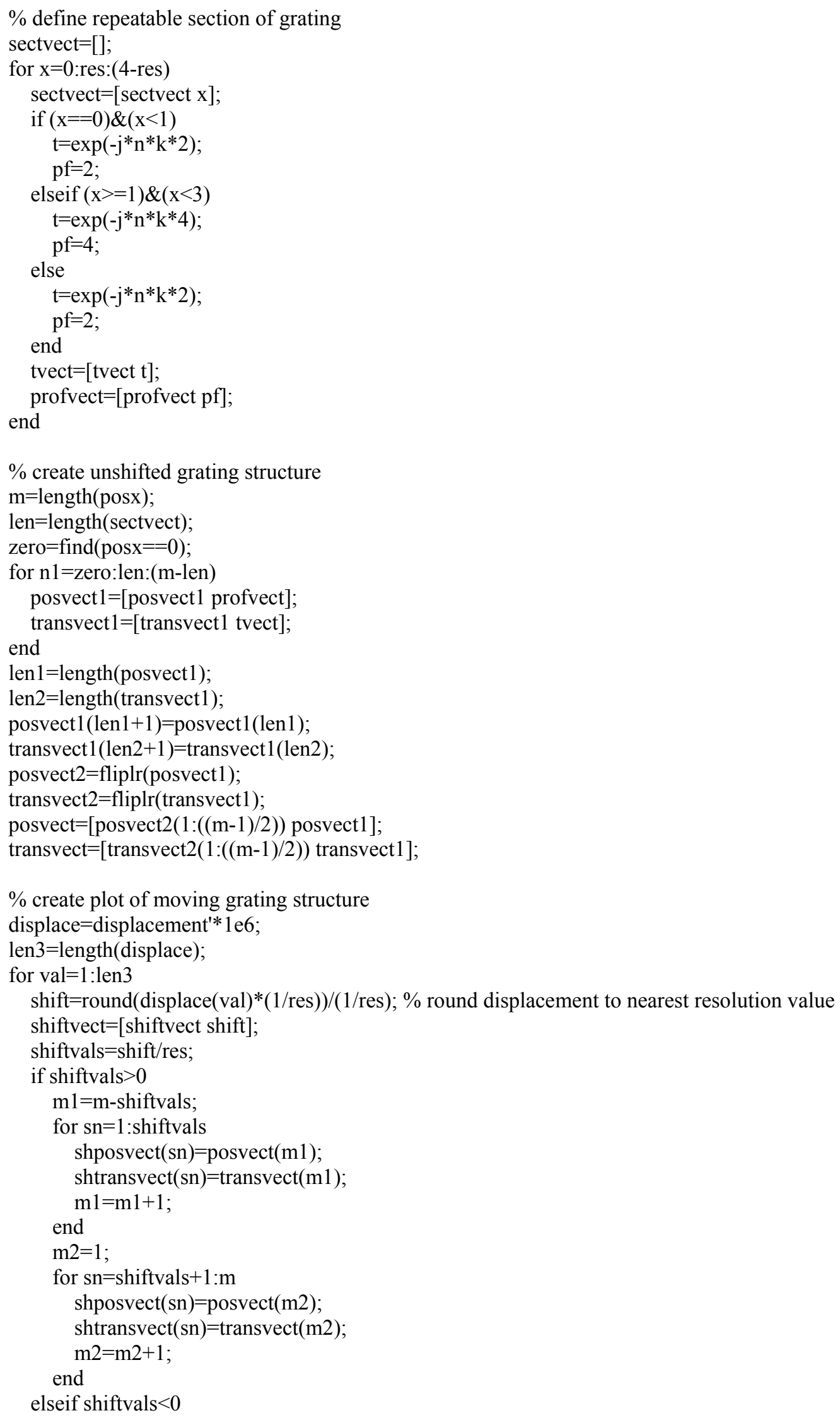




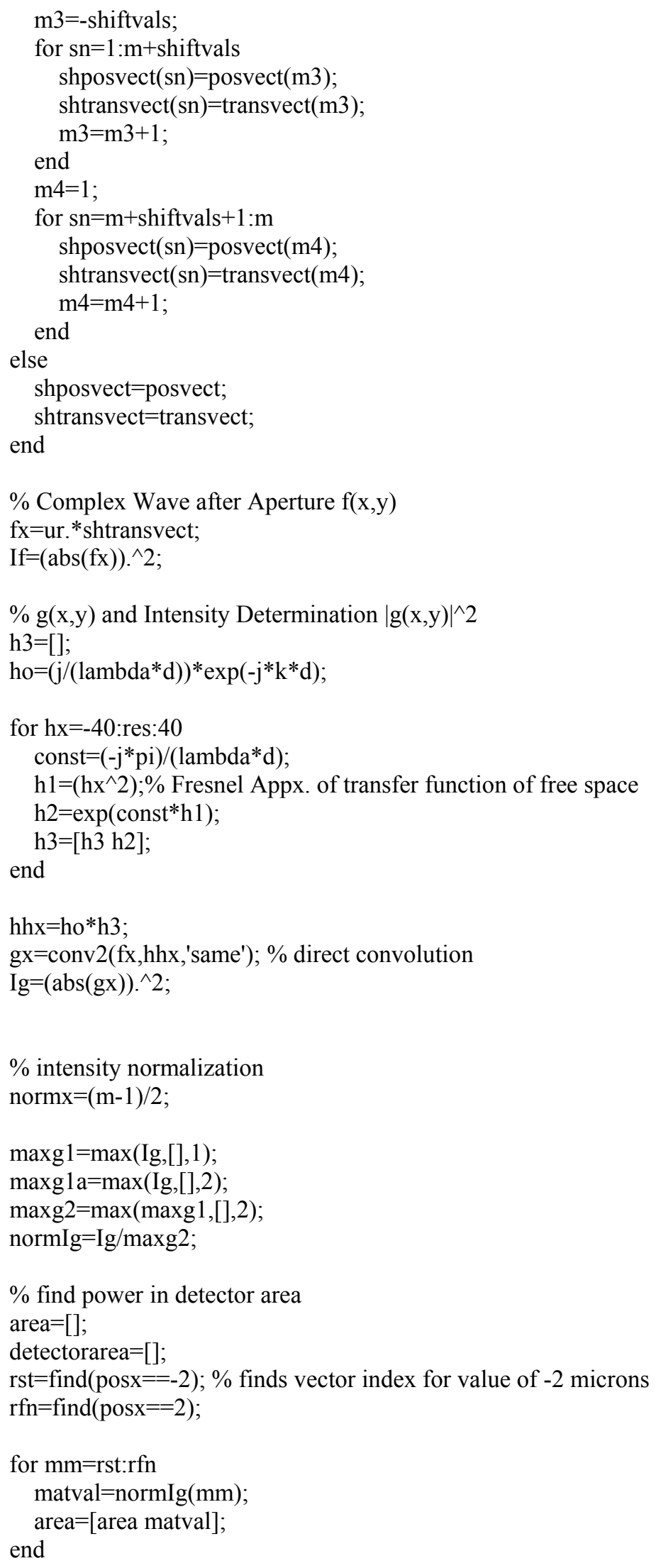




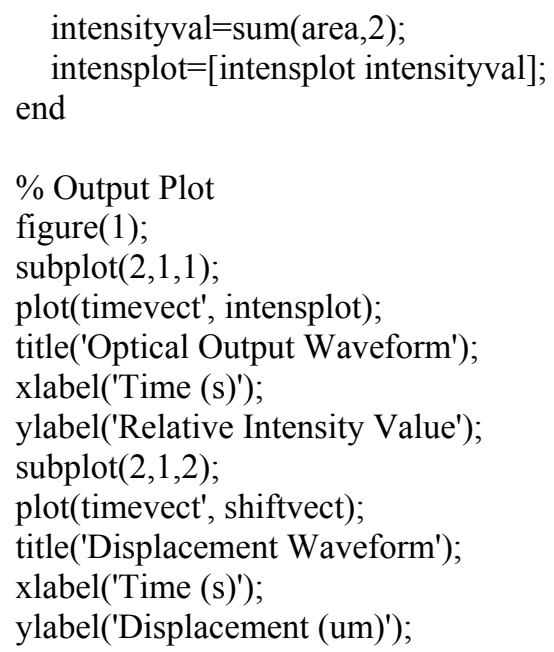




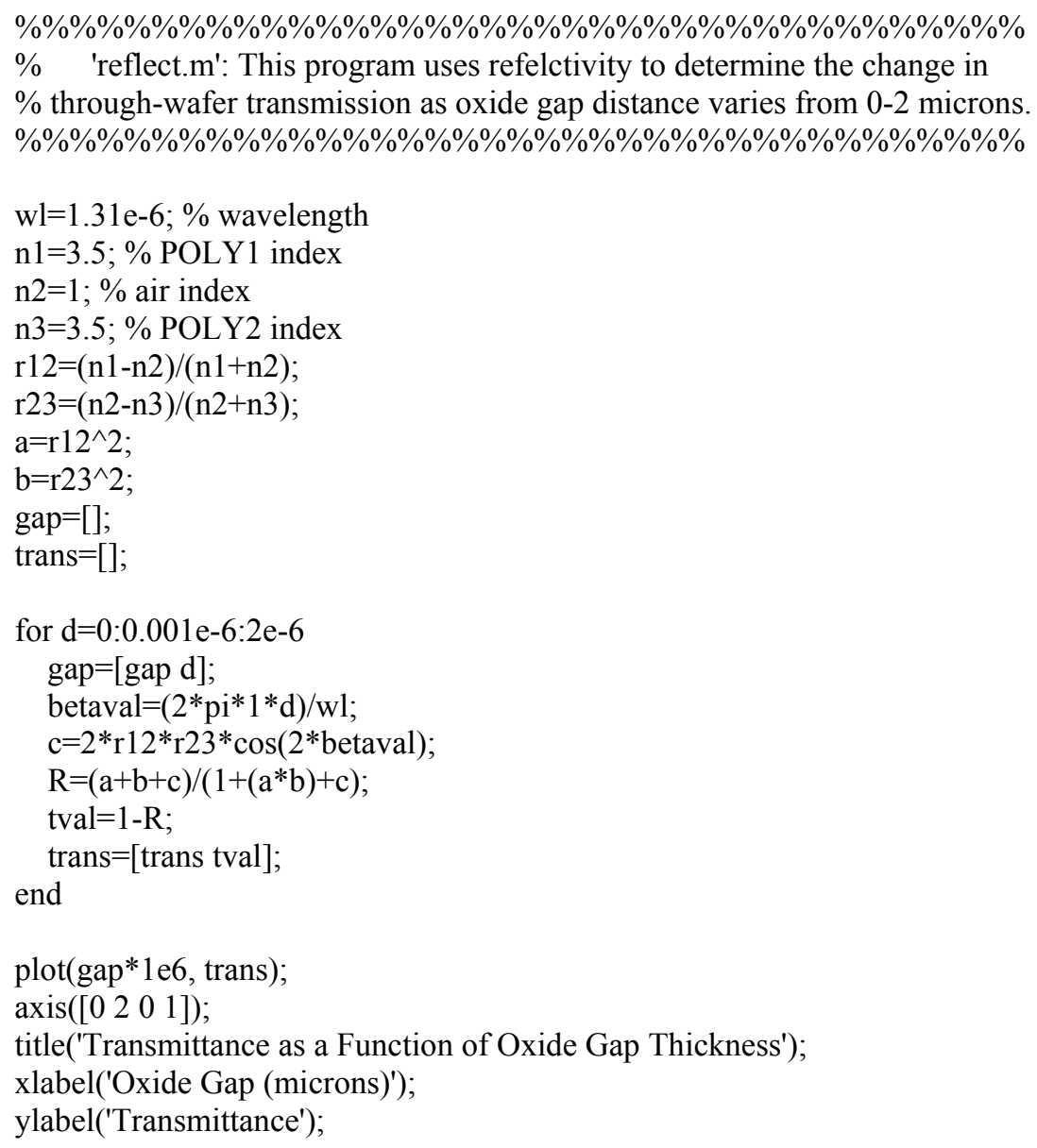


\%\%\%\%\%\%\%\%\%\%\%\%\%\%\%\%\%\%\%\%\%\%\%\%\%\%\%\%\%\%\%\%\%\%\%\%

$\% \quad$ 'reflect2.m': This program uses reflectivity to determine the change

$\%$ in through-wafer transmission as the stage moves vertically and changes

$\%$ the oxide gap distance at a frequency of $1 \mathrm{kHz}$.

\%\%\%\%\%\%\%\%\%\%\%\%\%\%\%\%\%\%\%\%\%\%\%\%\%\%\%\%\%\%\%\%\%\%\%\%

clear all;

$\mathrm{f}=1000 ; \%$ frequency in $\mathrm{Hz}$

$\mathrm{wl}=1.31 \mathrm{e}-6 ; \%$ wavelength

$\mathrm{n} 1=3.5 ; \%$ POLY1 index

$\mathrm{n} 2=1 ; \%$ air index

$\mathrm{n} 3=3.5 ; \%$ POLY2 index

$\mathrm{r} 12=(\mathrm{n} 1-\mathrm{n} 2) /(\mathrm{n} 1+\mathrm{n} 2)$

$\mathrm{r} 23=(\mathrm{n} 2-\mathrm{n} 3) /(\mathrm{n} 2+\mathrm{n} 3)$

$\mathrm{a}=\mathrm{r} 12^{\wedge} 2$;

$\mathrm{b}=\mathrm{r} 23^{\wedge} 2$;

time $=[]$;

gap $=[]$;

trans $=[]$;

for $\mathrm{t}=0: 1 \mathrm{e}-6: 0.0025$

time $=[$ time t $]$;

$\mathrm{d}=0.05 \mathrm{e}-6 * \sin (2 *$ pi*f*t);

$\mathrm{h}=\mathrm{d}+1.75 \mathrm{e}-6$;

gap $=$ gap h];

betaval $=\left(2 * \mathrm{pi}^{*} 1 * \mathrm{~h}\right) / \mathrm{wl}$;

$\mathrm{c}=2 * \mathrm{r} 12 * \mathrm{r} 23 * \cos (2 *$ betaval $)$;

$\mathrm{R}=(\mathrm{a}+\mathrm{b}+\mathrm{c}) /(1+(\mathrm{a} * \mathrm{~b})+\mathrm{c})$;

tval $=1-\mathrm{R}$;

trans $=[$ trans tval];

end

$\%$ Output Plot

figure(1);

subplot $(2,1,1)$;

plot(time, gap*1e6);

title('Vertical Variation of Oxide Gap Thickness');

xlabel('Time (s)');

ylabel('Gap Distance (microns)');

subplot $(2,1,2)$;

plot(time, trans);

title('Transmittance Variation with Vertical Stage Motion');

xlabel('Time (s)');

ylabel('Transmittance'); 


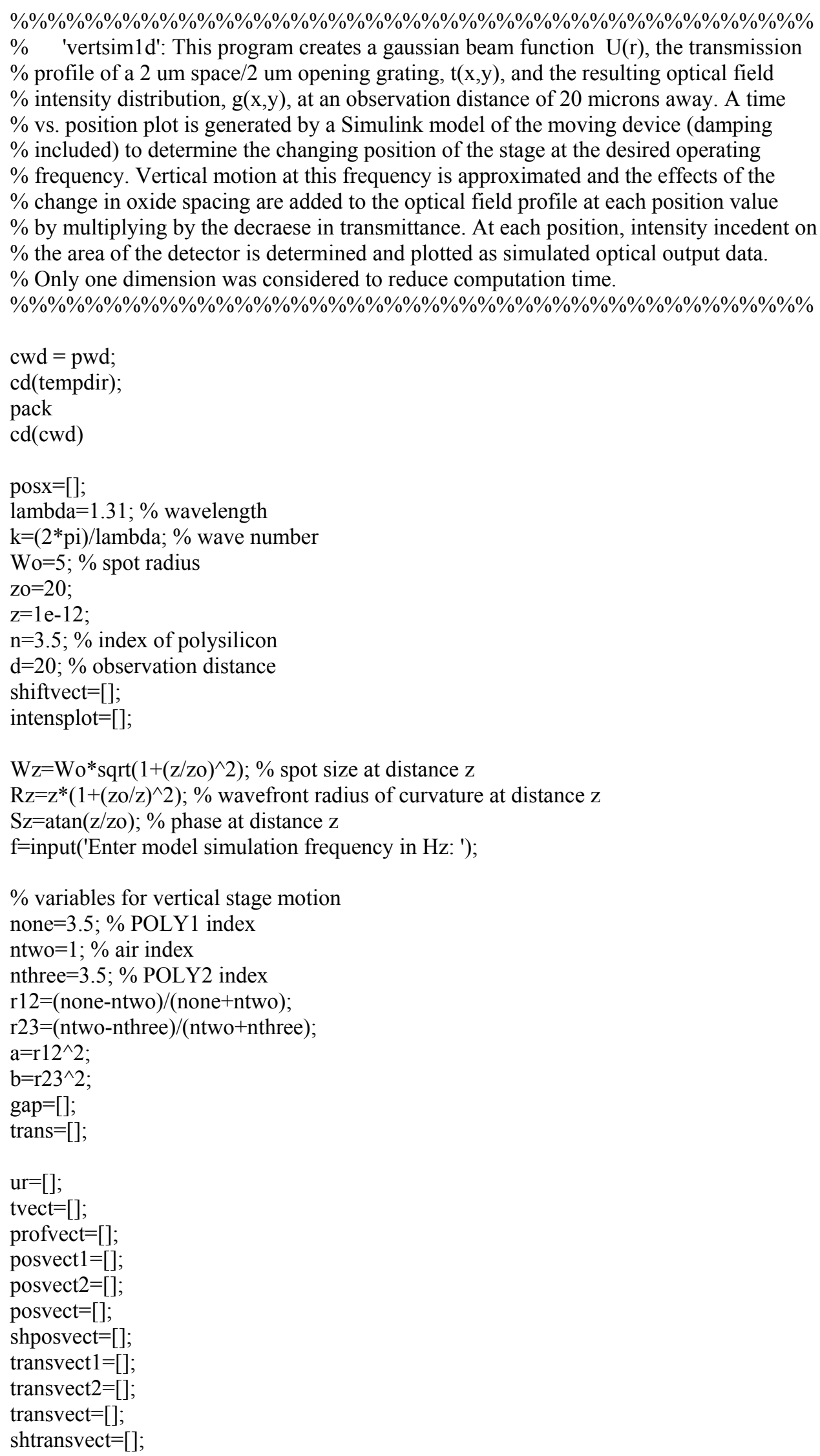




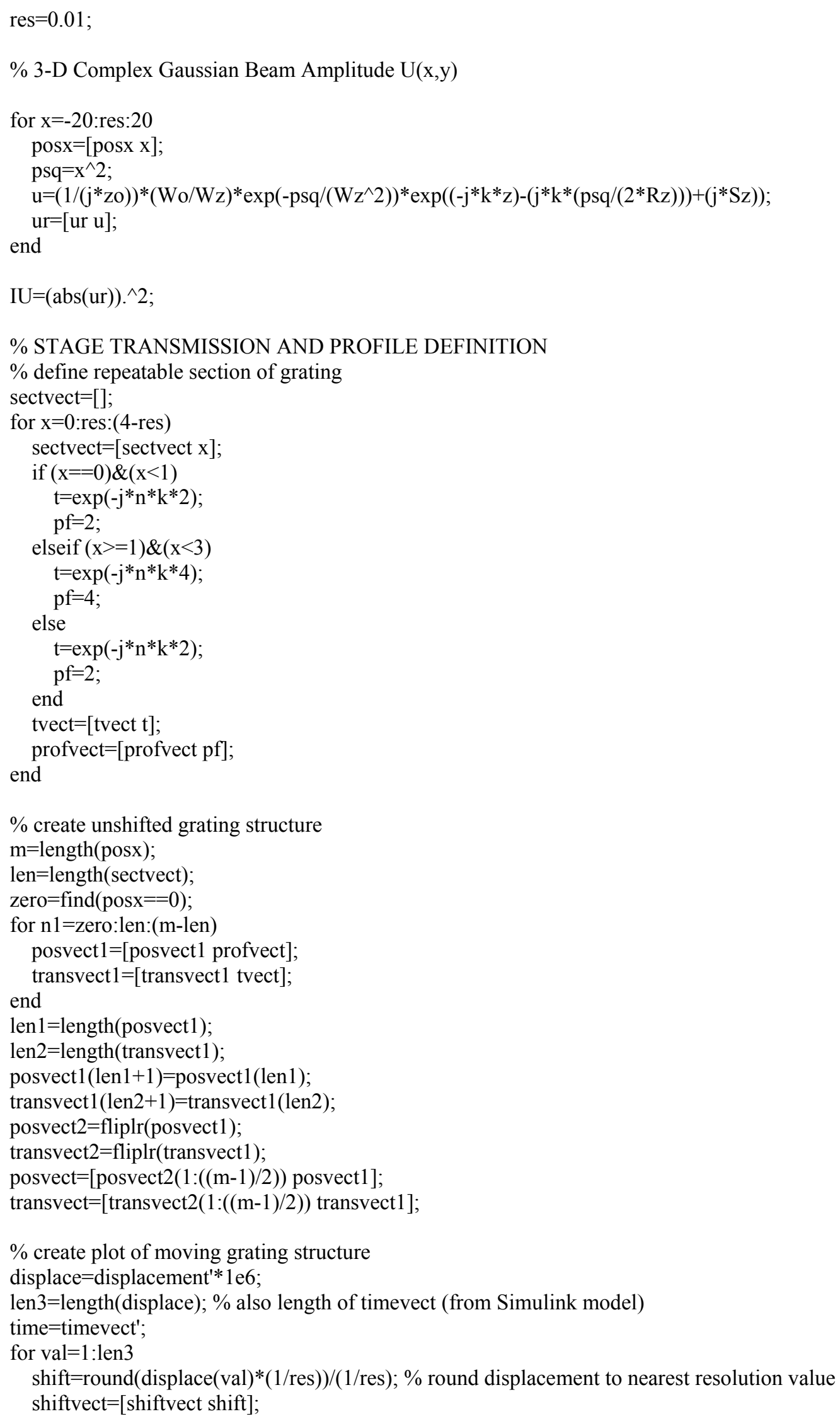




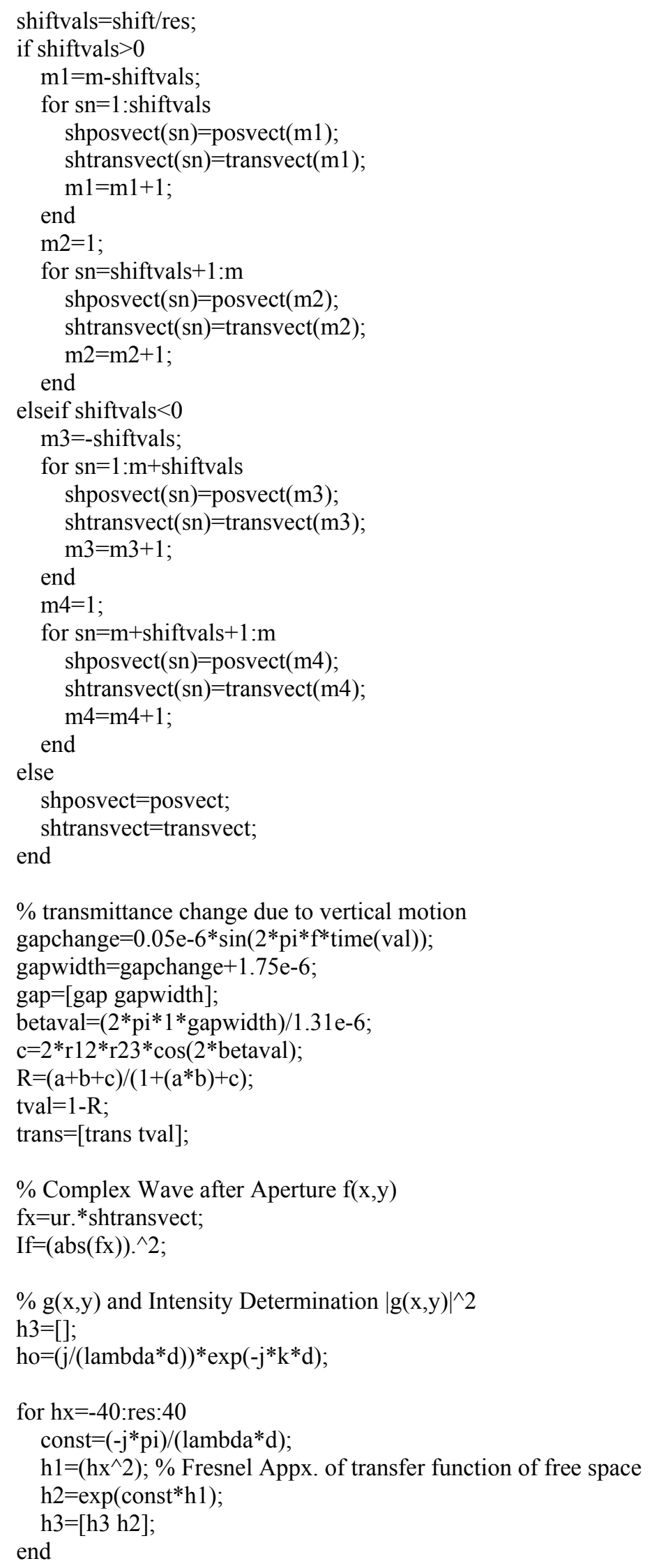




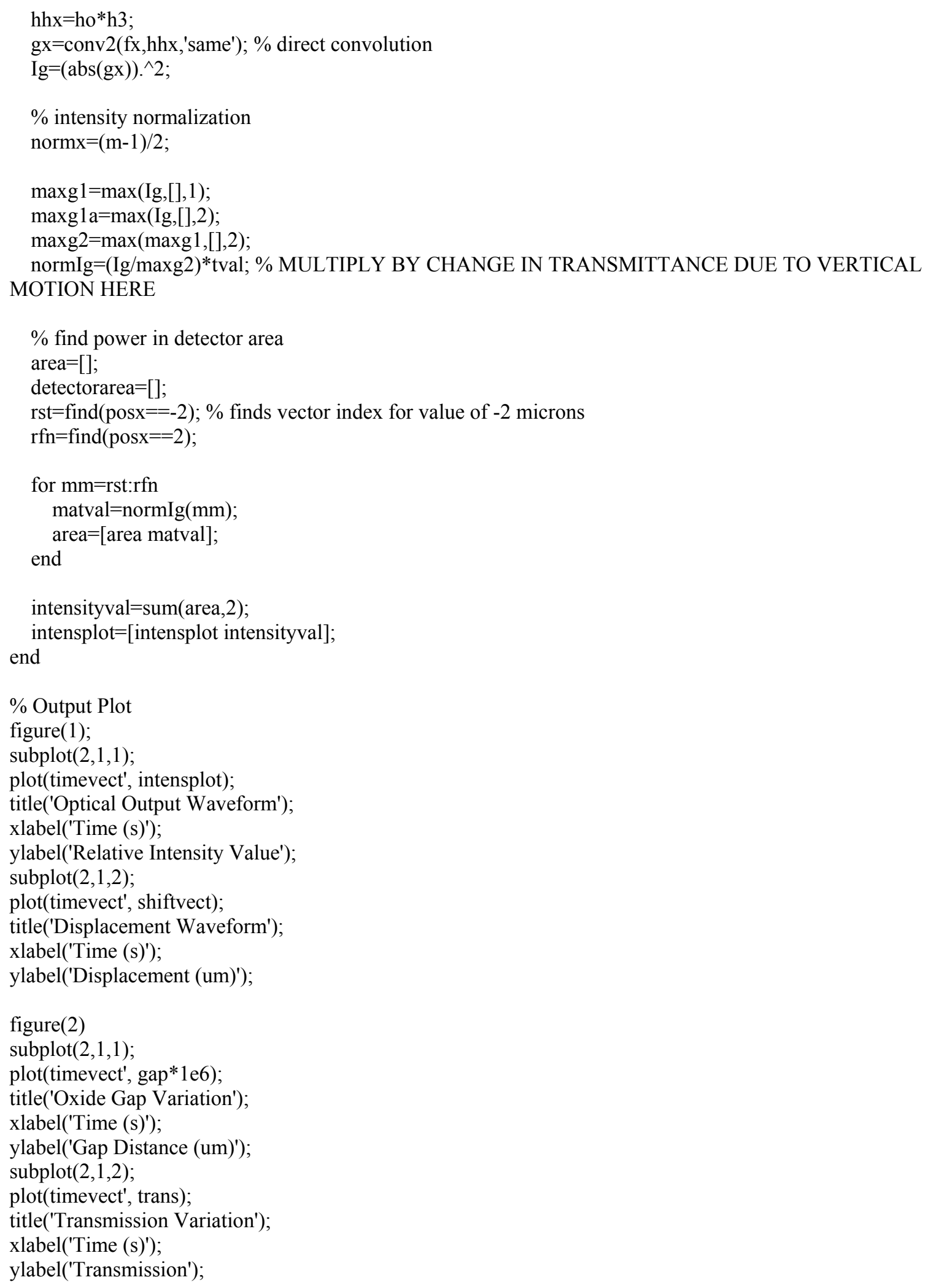




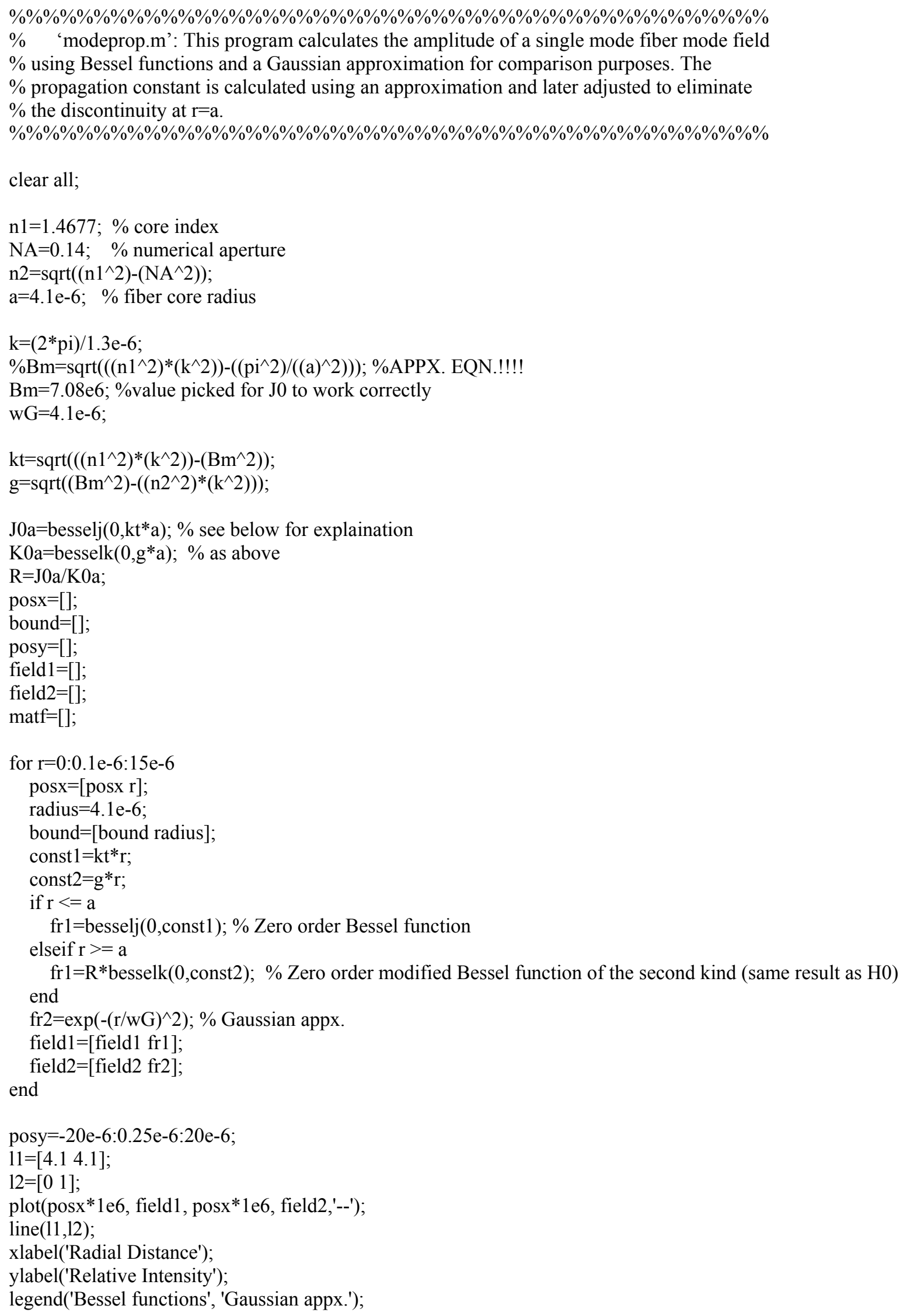




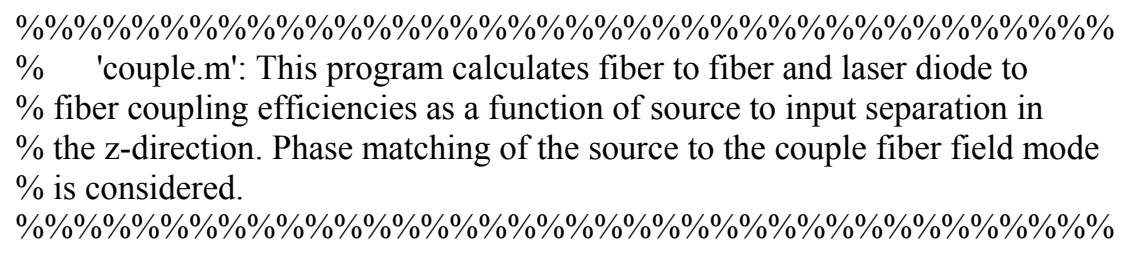

clear all;

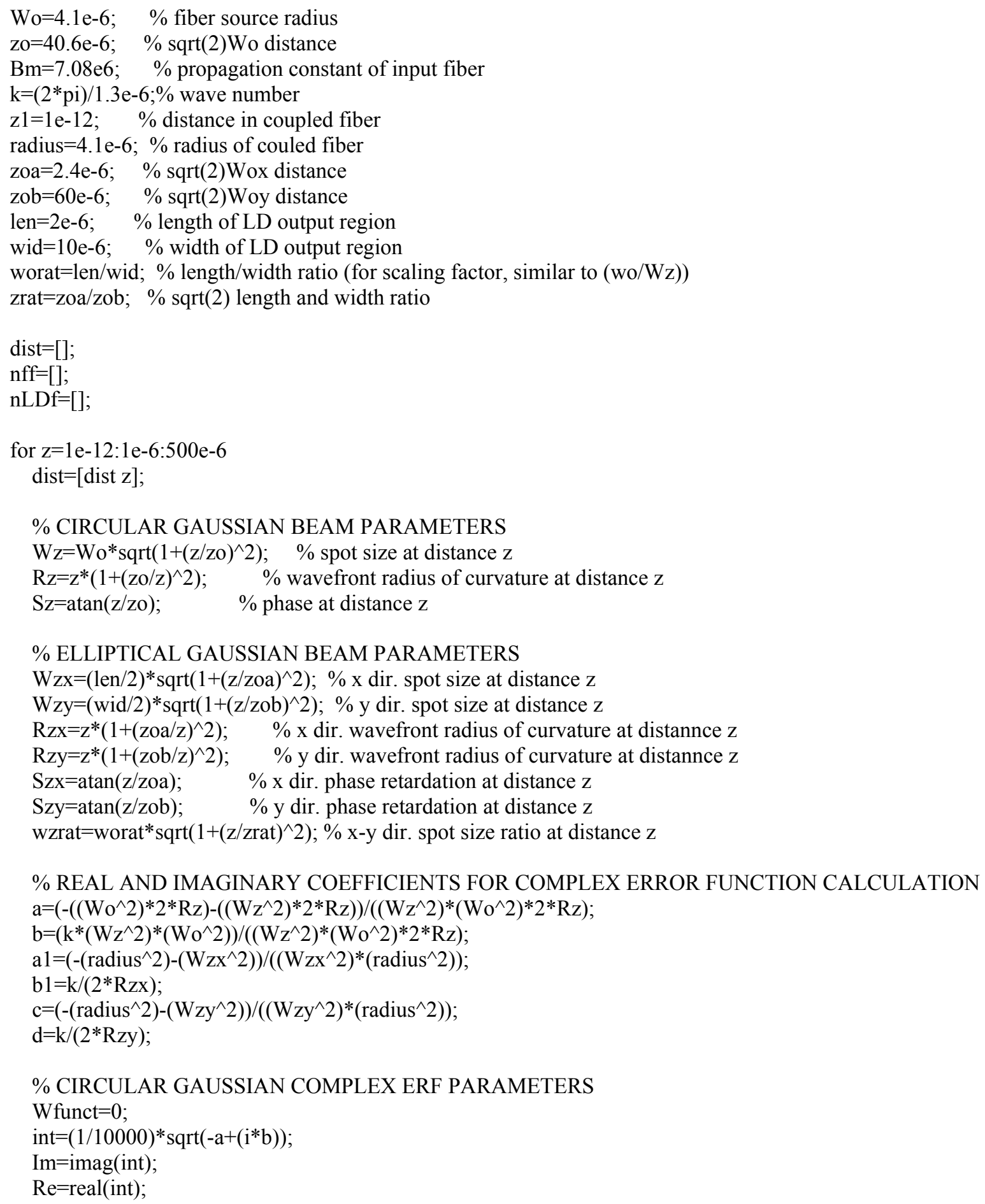




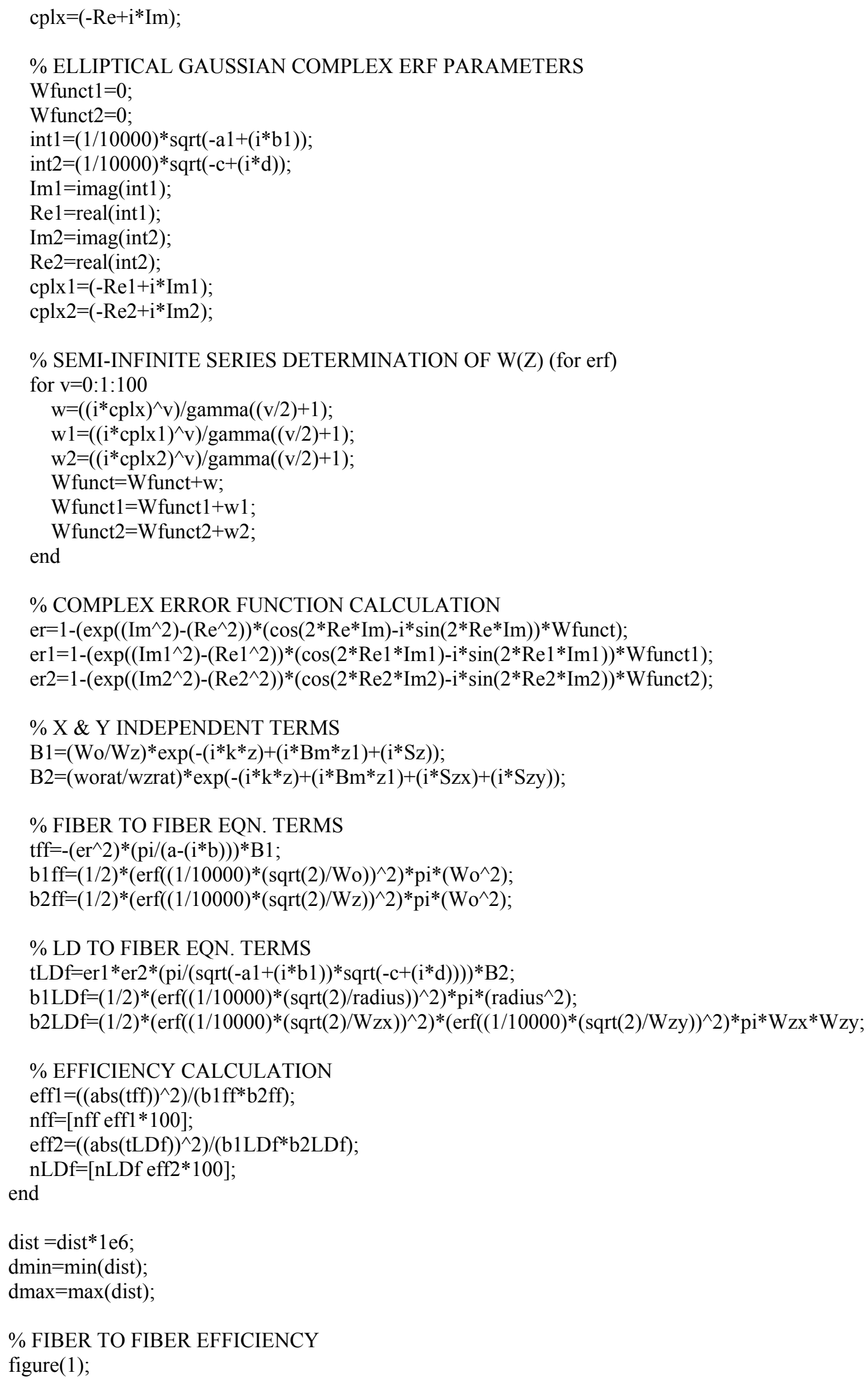




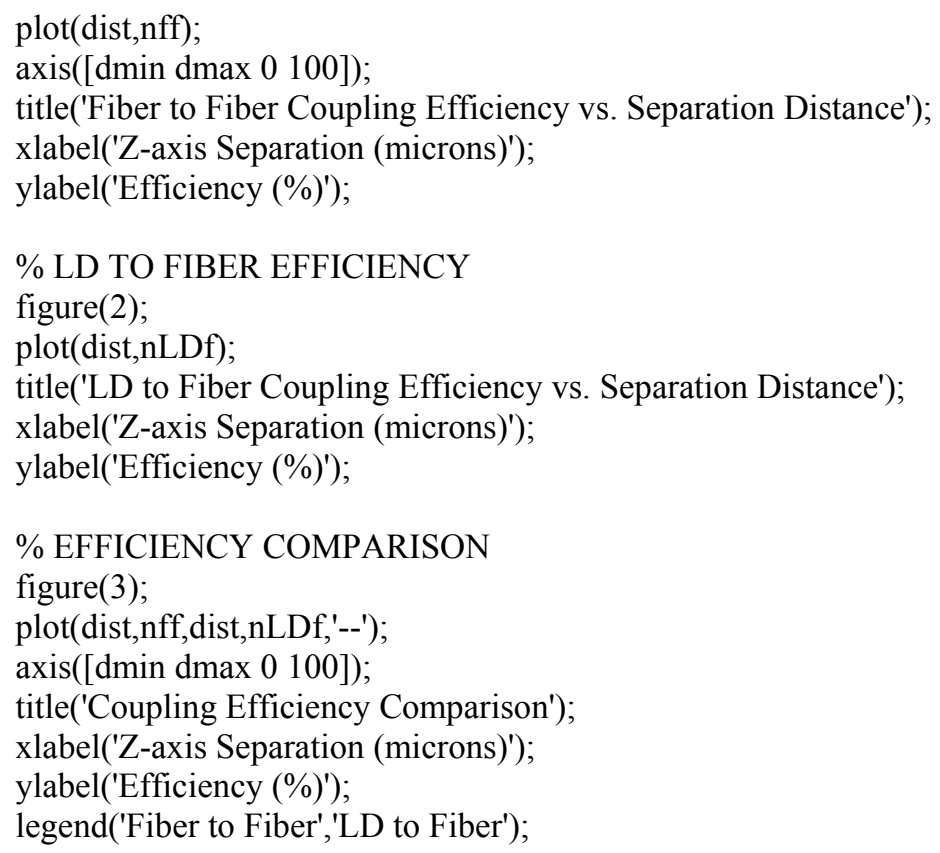




\section{Bibliography}

[1] F. Merat and M. Mehregany, "Integrated micro-opto-electro-mechanical systems," Proc. SPIE MicroOptics/Micromechanics and Laser Scanning and Shaping, Vol. 2383, pp. 88-98, May 1995.

[2] Peterson, Kurt, "Silicon as a Mechanical Material," Proc. IEEE, Vol. 70, No. 5, pp. 420-457, May 1982.

[3] Bourne, Marlene, Cahners In-Stat Group, as quoted in: Roberts, Bill, "MEMS the Word," Electronic Business, (July 2001), http://www.e-insite.net/eb-mag/index.asp?layout=article\&articleId=CA89894.

[4] "Microengine comb drive," "24-bit mechanical lock," and "Microengine drive gear," Web Page, http://mems.sandia.gov/scripts/images.asp, (2001).

[5] F. M. Dickey, S. C. Holswade, L. A. Hornak, and K. S. Brown, "Optical Methods for Micromachine Monitoring and Feedback," Sensors and Actuators A - Physical, Vol. 78, p. 220 (1999).

[6] L. Dellmann, T. Akiyama, D. Briand, S. Gautsch, O. Guenat, B, Guldimann, P. Luginbuhl, C. Marxer, U. Staufer, B. van der Schoot, and N.F. de Rooij, "Microsystems for diverse applications using recently developed microfabrication techniques," Proc. SPIE MOEMS and Miniaturized Systems, Vol. 4178, pp. 16-27, Sept. 2000.

[7] M.E. Motamedi, M.C. Wu, and K.S. Pister, "Micro-opto-electro-mechanical devices and on-chip optical processing,” Optical Engineering, Vol. 36, No. 5, pp. 1282-1297, May 1997.

[8] J. A. Walker, “Telecommunications Applications of MEMS," MST News, No. 3/00, pp. 6-9, June 2000.

[9] L.J. Hornbeck, “The DMD ${ }^{\mathrm{TM}}$ Projection Display Chip: A MEMS Based Technology," MRS Bulletin, Vol. 26, No. 4, pp. 325-327, April 2001.

[10] D.W. Wine, M.P. Helsel, L. Jenkins, H. Urey, T.D. Osborn, "Performance of a biaxial MEMS-based scanner for microdisplay applications," Proc. SPIE MOEMS and Miniaturized Systems, Vol. 4178, pp. 186-195, Sept. 2000.

[11] A. Gehner, M. Wildenhain, and H. Lakner, "Micromirror arrays for wavefront correction," Proc. SPIE MOEMS and Miniaturized Systems, Vol. 4178, pp. 348-357, Sept. 2000.

[12] T.G. Bifano, R.K. Mail, J.K. Dorton, J. Perreault, N. Vandelli, M.N. Horenstein, D.A. Castañon, "Continuous-membrane surface-micromachined silicon deformable mirror," Optical Engineering, Vol. 36, No. 5, pp. 1354-1360, May 1997.

[13] See e.g., M.S. Rodgers, J.J. Sniegowski, S.L. Miller, and G.F. LaVigne, "Designign and Operating Electrostatically Driven Microengines," Proc. $44^{\text {th }}$ International Instrumentation Symposium, Reno, NV, pp. 56-65, May 1998. 
[14] G.K. Fedder and Q. Jing, "Hierarchical circuit-level design methodology for microelectromechanical systems," IEEE Trans. On Circuits and Systems II: Analog and Digital Signal Processing, V46, No. 10, pp. 1309-1315, 1999.

[15] H.H. Pham and A. Nathan, "Compact MEMS-SPICE Modeling," Sensors and Materials, Vol. 10, No. 2, pp. 63-75, 1998.

[16] D. Gibson, C. Purdy, and A. Hare, "Design automation of MEMS systems using behavioral modeling," Proc. IEEE Great Lakes Symp. On VLSI, Ann Arbor, MI, pp. 266-269, March 1999.

[17] A. Deway, H. Ren, and T. Zhang, "Behavioral modeling of microelectromechanical systems (MEMS) with statistical performance-variability reduction and sensitivity analysis," IEEE Trans. On Circuits and Systems II: Analog and Digital Signal Processing, Vol. 47, No. 2, pp. 105-113, 2000.

[18] G. K. Fedder, "Simulation of Microelectromechanical Systems," PhD. Thesis, University of California at Berkeley (1994).

[19] J.J. Allen, S.L. Miller, G.F. LaVigne, and M.S. Rodgers, "Dynamic Effects of Linkage Joints in Electrostatic Microengines," Proc. Modeling and Simulation of Microsystems, Santa Clara, CA, April 1998.

[20] P. R. Nelson, P. B. Chu, and K. S. J. Pister, "Optical methods for characterization of MEMS device motion," Proc. SPIE Microlithography and Metrology in Micromachining, Vol. 2640, pp. 53-57, Sept. 1995.

[21] J. S. Burdess, A.J. Harris, D. Wood, R. J. Pitcher, and D. Glennie, "A System for the Dynamic Characterization of Microstructures," Journal of Microelectromechanical Systems, Vol. 6, Vo. 4, pp. 322-328, Dec. 1997.

[22] W. D. Cowan, V. M. Bright, and G. C. Dalton, "Measuring frequency response of surface-micromachined resonators," Proc. SPIE Microlithography and Metrology in Micromachining III, Vol. 3225, pp. 32-43, Sept 1997.

[23] G. K. Fedder and R. T. Howe, "Multimode Digital Control of a Suspended Polysilicon Microstructure," Journal of Microelectromechanical Systems, Vol. 5, No. 4, pp. 283-297, Dec. 1996.

[24] D.M. Freman, "Measuring Motions of MEMS," MRS Bulletin, Vol. 26, No. 4, pp. 305-306, April 2001.

[25] A.T.T.D. Tran, J.J. Lee, and K. Zhang, "Ultrafine motion detection of Micromechanical structures using optical Moiré pattern," IEEE Photonics Tech. Letters, Vol. 8, No. 8, Aug. 1996.

[26] D.J. Burns and H.F. Helbig, "A system for automatic electrical and optical characterization of microelectromechanical devices," Journal of Microelectromechanical Systems, Vol. 8, No. 4, pp 473-482, Dec. 1999.

[27] S. Tadigadapa and N. Najafi, "Reliability of Microelectromechanical Systems (MEMS)," Proc. SPIE Reliability, Testing and Characterization of MEMS/MOEMS, Vol. 4558, pp. 197-205, Oct. 2001.

[28] C. Marxer, M.A. Gretillat, N.F, de Rooij, R. Battiq, O. Anthamatten, B, Valk, and P. Vogel, "Reliability considerations for electrostatic polysilicon actuators using as an example the REMO Component," Sensors and Actuators A: Physical, Vol. 61, No. 1-3, pp. 449-454, June 1997.

[29] M. Mehregany, P. Nagarkar, S.D. Senturia, and J.H. Lang, "Operation of microfabricated harmonic and ordinary side-drive motors," Proc. IEEE Workshop on Micro Electro Mechanical Systems, Napa Valley, CA, pp 1-8, Feb. 1990.

[30] J.A. Walraven, S.S. Mani, J.G. Fleming, T.J. Headley, P.G. Kotula, A.A. Pimentel, M.J. Rye, D.M. Tanner, and N.F. Smith, "Failure analysis of tungsten coated polysilicon micromachined microengines," Proc. SPIE MEMS Reliability for Critical Applications, Vol. 4180, pp. 49-57, Sept. 2000.

[31] M.R. Douglass, "Lifetime Estimates and Unique Failure Mechanisms of the Digital Micromirror Device (DMD)," Proc. $36^{\text {th }}$ Annual Intl. Reliability Physics Symposium, Reno, NV, pp. 9-16, 1998. 
[32] See e.g. Dickey, Holswade, Christenson, Garcia, and Polosky, "Optical measurement of LIGA milliengine performance," Proc. SPIE Miniaturized Sys. With Micro-Optics and Micromechanics III, Vol. 3276, pp. 28-36 (1998).

[33] E.J. Garcia and J.J. Sniegowski, "Surface micromachined microengine," Sensors and Actuators A: Physical, Vol. 48(3), pp. 203-14 (1995).

[34] M.P. de Boer and T.M. Mayer, “Tribology of MEMS," MRS Bulletin, Vol. 26, No. 4, pp. 302-304, April 2001.

[35] P. May, S. T. Wilkinson, N. M. Jokerst, D. S. Wills, M. Lee, O. Vendier, S. W. Bond, Z. Hou, G. Dagnall, M. A. Brooke, A. Brown, and E. Schenfeld, "Design issues for through-wafer optoelectronic multicomputer interconnects," Proc. Second International Conference on Massively Parallel Processing Using Optical Interconnections, San Antonio, TX, pp.8-15, Oct. 1995.

[36] J.M. Dawson, “Through-Wafer Interrogation of MEMS Device Motion," Electrical Engineering M.S. thesis. West Virginia University, July 1999.

[37] K.S. Brown, "On the Feasibility of Integrated Optical Waveguide-Based In-Situ Monitoring of Microelectromechanical Systems (MEMS)," Electrical Engineering Ph.D. dissertation, West Virginia University, Aug. 2000.

[38] J. M. Dawson, J. Chen, K. S. Brown, P. Famouri, and L. A. Hornak, "Through-Wafer Optical Probe Characterization for MEMS Positional State Monitoring and Feedback Control," Optical Engineering, Vol. 39, No. 12, pp., Dec. 2000.

[39] V. Barger and M. Olsson, Classical Mechanics A Modern Perspective, McGraw-Hill Book Co, New York (1994).

[40] A. Kolpekwar and R. D. Blanton, "Development of a MEMS Testing Methodology," IEEE International Test Conference, pp. 923-31 (1997).

[41] Y. Cho, et. al., "Viscous Damping Model for Laterally Oscillating Microstructures," IEEE Journal of Microelectromechanical Systems, Vol. 3, No. 2, pp. 81-86 (1994).

[42] T. D. Milster, "Optical Servo Signal Generation Techniques for MEMS Applications", SPIE Symposium on Miniaturized Systems with Micro-Optics and MEMS, Santa Clara, CA (Sept. 1999).

[43] M. Ferstl and A-M. Frisch, "Static and dynamic Fresnel zone lenses for optical interconnections," Journal of Modern Optics, Vol. 43, no. 7, pp. 1451-1462 (1996).

[44] L.A. Hornak, "Fresnel phase plate lenses for through-wafer OI," Applied Optics, Vol. 26, No. 17, pp. 364954 (1987).

[45] L. Wang, J.M. Dawson, J. Chen, P. Famouri, and L.A. Hornak, "Stroke-length control of a MEMS device," Proc. 2000 IEE International Symp. on Industrial Electronics, 2, pp. 535-539, Dec. 2000.

[46] P. Famouri, L Wang, J.M. Dawson, and L.A. Hornak, "Optical monitoring-based real-time feedback control of MEMS comb resonator," submitted to IEEE Electronics Letters.

[47]A. Kolpekwar and R. D. Blanton, "Development of a MEMS Testing Methodology," IEEE International Test Conference, pp. 923-31 (1997).

[48]G. K. Fedder, "Simulation of Microelectromechanical Systems," PhD. Thesis, University of California at Berkeley (1994).

[49] E. Hecht, Optics, Addison-Wesley Publishing Co., Redding, Mass. (1987).

[50] B.E.A Saleh and M.C. Teich, Fundamentals of Photonics, Wiley-Interscience, New York (1991).

[51] G.J. Swanson and W.B. Veldkamp, "Diffractive optical elements for use in infrared systems," Optical Engineering, Vol. 28, No. 6, pp. 605-608, June 1989.

[52] C.J. Smith, Optics, Edward Arnold, Ltd., Baltimore (1960). 
[53] L. d'Auria, J.P. Huignard, A.M. Roy, and E. Spitz, "Photolithographic fabrication of thin film lenses," Optics Communications, Vol. 5, No. 4, pp.232-235, July 1972.

[54] G. Hatakoshi and K, Goto, "Grating lenses for the semiconductor wavelength," Applied Optics, Vol. 24, No. 24, pp. 4307-4311, Dec. 1985.

[55] M. Young, "Zone Plates and Their Aberrations," Journal of the Optical Society of America, Vol. 62, No. 8, pp-972-976, Aug. 1972.

[56] A.B. Buckman, Guided Wave Photonics, Saunders College Publishing, New York (1992).

[57] Web site, http://www.breault.com/ftp/docs/procnotes/bropn1152_telecom.pdf.

[58] R.E. Wagner and W.J. Tomlinson, "Coupling efficiency of optics in single-mode fiber components," Applied Optics, Vol. 21, No. 15, pp. 2671-2688, Aug. 1982.

[59] Corning fiber data sheets, Web site, http://www.corningfiber.com/products/smf28_frame.htm.

[60] "MUMPS design handbook," Web Page, http://www.memsrus.com/cronos/mumps.pdf, (2002) 